\title{
3-Fluoro-4-hydroxyprolines: Synthesis, conformational analysis and stereoselective recognition by the VHL E3 ubiquitin ligase for targeted protein degradation
}

Andrea Testa $^{\mathrm{a}}$, Xavier Lucas ${ }^{\mathrm{a}}$, Guilherme V. Castro ${ }^{\mathrm{a}}$, Kwok-Ho Chan ${ }^{\mathrm{a}}$, Jane E. Wright ${ }^{\mathrm{a}}$, Andrew C. Runcie ${ }^{a}$, Morgan S. Gadd ${ }^{a}, \dagger$, William T. A. Harrison ${ }^{b}$, Eun-Jung Ko ${ }^{c}$, Daniel Fletcher $^{\mathrm{c}}$, Alessio Ciulli ${ }^{\mathrm{a}, *}$

${ }^{a}$ Division of Biological Chemistry and Drug Discovery, School of Life Sciences, University of Dundee, James Black Centre, Dow Street, Dundee DD1 5EH, Scotland, U.K.

${ }^{b}$ Department of Chemistry, University of Aberdeen, Meston Walk, Aberdeen AB24 3UE, Scotland, U.K.

${ }^{c}$ Drug Discovery Unit, Division of Biological Chemistry and Drug Discovery, School of Life Sciences, University of Dundee, James Black Centre, Dow Street, Dundee DD1 5EH, Scotland, U.K.

\section{Supporting information}

Contents

Supplementary tables

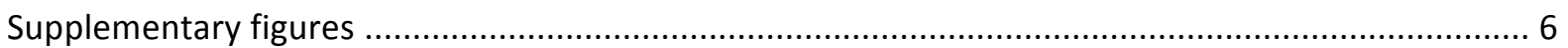

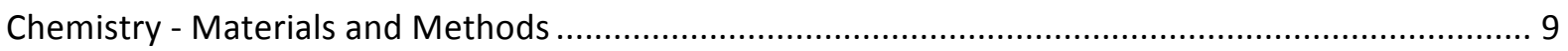

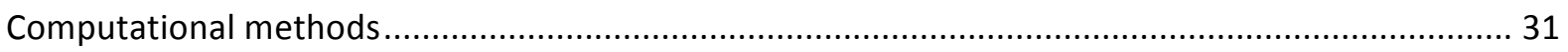

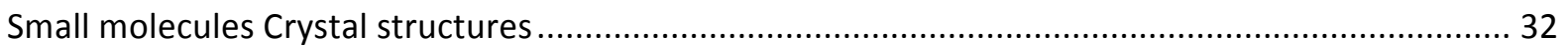

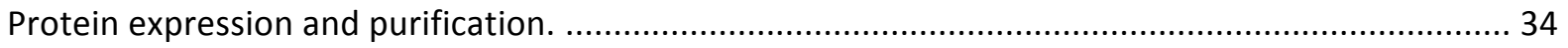

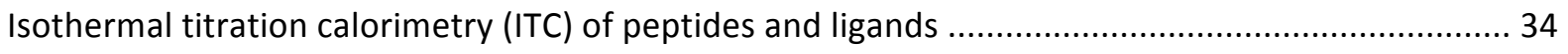

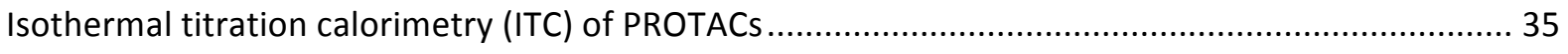

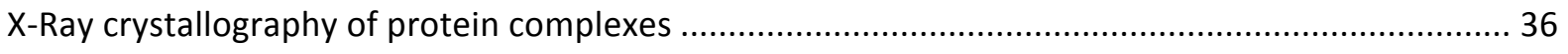

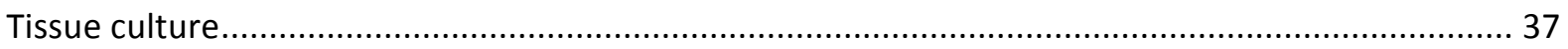

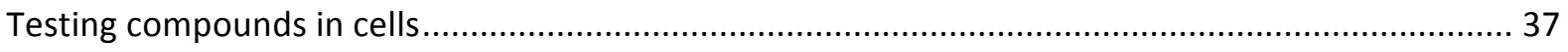

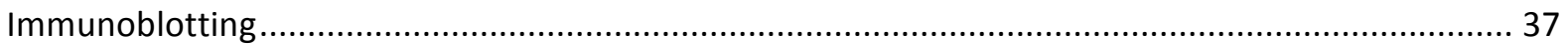

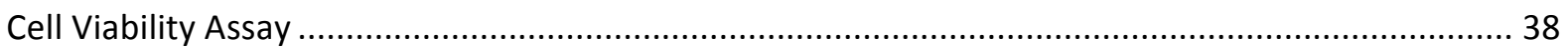

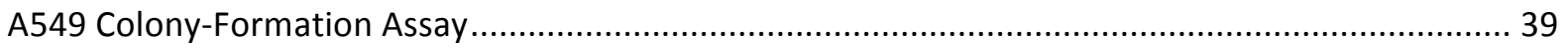

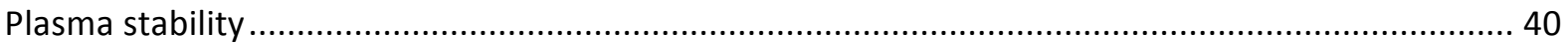

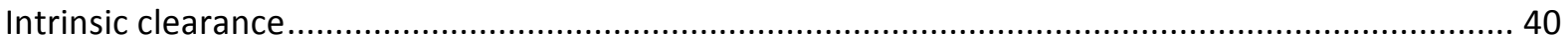

Parallel Artificial Membrane Permeability Assay (PAMPA) ........................................................... 41 
CHI Log D determination

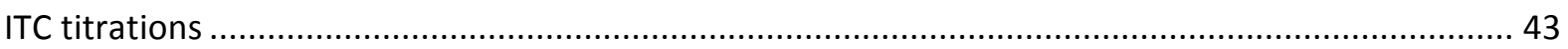

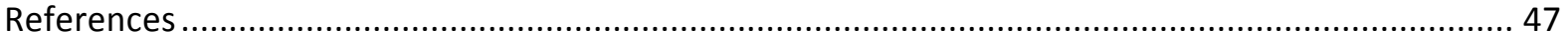

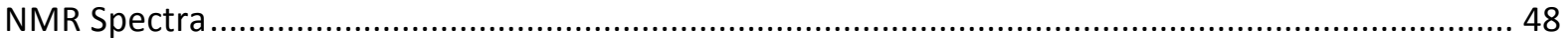




\section{Supplementary tables}

Supplementary Table 1. Preliminary evaluation of different DFT methods to correctly model reference compounds Ac-Pro-OMe, Ac-3-fpro-OMe and Ac-Hyp-OMe in water solvation continuum.

\begin{tabular}{|c|c|c|c|c|c|c|c|}
\hline Level of theory & Compound & $E$ (Hartree) & $\begin{array}{c}\mathrm{ZPE} \\
(\mathrm{kcal} / \mathrm{mol})\end{array}$ & $\begin{array}{l}E_{\text {corrected }} \\
\text { (Hartree) }\end{array}$ & $\begin{array}{l}\text { Imaginary } \\
\text { frequencies }\end{array}$ & $\begin{array}{l}\text { Predicted } \\
\text { ratio } \\
\text { endo:exo }\end{array}$ & $\begin{array}{c}\text { Known major } \\
\text { pucker }\end{array}$ \\
\hline \multirow{2}{*}{ B3LYP-D3/aug-cc-pVTZ(-F) } & $\begin{array}{c}\text { Ac-Hyp-OMe } \\
\mathrm{C}^{4} \text { endo }\end{array}$ & -668.6375 & 134.603 & -668.4230 & 0 & \multirow{2}{*}{$52: 48$} & \multirow[b]{2}{*}{ exo } \\
\hline & \begin{tabular}{|c|} 
Ac-Hyp-OMe \\
$\mathrm{C}^{4}$ exo
\end{tabular} & -668.6373 & 134.541 & -668.4229 & 0 & & \\
\hline \multirow{2}{*}{$\begin{array}{c}\text { B3LYP-D3/RAPPOPORT- } \\
\text { SVPD }\end{array}$} & $\begin{array}{c}\text { Ac-Pro-OMe } \\
\mathrm{C}^{4} \text { endo }\end{array}$ & -592.7794 & 131.585 & -592.5697 & 0 & \multirow{2}{*}{$49: 51$} & \multirow{2}{*}{ endo } \\
\hline & \begin{tabular}{|c|} 
Ac-Pro-OMe \\
$\mathrm{C}^{4}$ exo
\end{tabular} & -592.7795 & 131.584 & -592.5698 & 0 & & \\
\hline \multirow{2}{*}{ M06-2X-D3/aug-cc-pVTZ(-F) } & $\begin{array}{c}\text { Ac-Hyp-OMe } \\
\mathrm{C}^{4} \text { endo }\end{array}$ & -668.3536 & 136.414 & -668.1362 & 0 & \multirow{2}{*}{$50: 50$} & \multirow{2}{*}{ exo } \\
\hline & \begin{tabular}{|c|} 
Ac-Hyp-OMe \\
$\mathrm{C}^{4}$ exo
\end{tabular} & -668.3536 & 136.438 & -668.1362 & 0 & & \\
\hline \multirow{2}{*}{ B97-D3/aug-cc-pVTZ(-F) } & \begin{tabular}{|c|} 
Ac-Hyp-OMe \\
$\mathrm{C}^{4}$ endo \\
\end{tabular} & -668.1780 & 131.450 & -667.9685 & 0 & \multirow{2}{*}{$54: 46$} & \multirow{2}{*}{ exo } \\
\hline & \begin{tabular}{|c|} 
Ac-Hyp-OMe \\
$\mathrm{C}^{4}$ exo
\end{tabular} & -668.1779 & 131.432 & -667.9684 & 0 & & \\
\hline \multirow{2}{*}{ PW6B95-D3/aug-cc-pVTZ(-F) } & $\begin{array}{c}\text { Ac-Hyp-OMe } \\
\mathrm{C}^{4} \text { endo }\end{array}$ & -669.4171 & 135.232 & -669.2016 & 0 & \multirow{2}{*}{$57: 43$} & \multirow{2}{*}{ exo } \\
\hline & \begin{tabular}{|c|} 
Ac-Hyp-OMe \\
$\mathrm{C}^{4}$ exo \\
\end{tabular} & -669.4168 & 135.231 & -669.2013 & 0 & & \\
\hline \multirow{2}{*}{ BP86-D3/aug-cc-pVTZ(-F) } & $\begin{array}{c}\text { Ac-Hyp-OMe } \\
\mathrm{C}^{4} \text { endo }\end{array}$ & -692.6534 & 122.551 & -692.4581 & 0 & \multirow{2}{*}{$56: 44$} & \multirow[b]{2}{*}{ exo } \\
\hline & \begin{tabular}{|c|} 
Ac-Hyp-OMe \\
$\mathrm{C}^{4}$ exo
\end{tabular} & -692.6529 & 122.409 & -692.4578 & 0 & & \\
\hline \multirow{2}{*}{ MN15/aug-cc-pVTZ(-F) } & $\begin{array}{c}\text { Ac-3-fpro- } \\
\text { OMe C } \text { endo }^{4}\end{array}$ & -691.8765 & 127.586 & -691.6732 & 0 & \multirow{2}{*}{$53: 47$} & \multirow{2}{*}{ exo } \\
\hline & $\begin{array}{c}\text { Ac-3-fpro- } \\
\text { OMe C }{ }^{4} \text { exo }\end{array}$ & -691.8762 & 127.494 & -691.6731 & 0 & & \\
\hline
\end{tabular}


Supplementary Table 2. Energetic values of F-Hyps 12a-d, Ac-Hyp-OMe, Ac-hyp-OMe, Ac-3-Fpro-OMe, Ac-3-fpro-OMe and Ac-Pro-OMe. DFT calculations were carried out at the MN15-L/aug-cc-pVTZ(-F) level of theory in water solvation continuum

\begin{tabular}{|c|c|c|c|c|c|c|c|c|}
\hline Compound & $\begin{array}{l}\text { Canonical } \\
\text { starting } \\
\text { pucker }\end{array}$ & $\begin{array}{l}\text { Optimized } \\
\text { pucker }\end{array}$ & $\begin{array}{c}E \\
\text { (Hartree) }\end{array}$ & $\begin{array}{c}\mathrm{ZPE} \\
(\mathrm{kcal} / \mathrm{mol})\end{array}$ & $\begin{array}{c}E_{\text {corrected }} \\
\text { (Hartree) }\end{array}$ & $\begin{array}{c}\text { Relative } \\
\Delta E \\
(\mathrm{kcal} / \mathrm{mol})\end{array}$ & $\begin{array}{c}\text { Fraction } \\
\text { of } \\
\text { conformer } \\
(\%)^{a}\end{array}$ & $\begin{array}{c}\text { Number of } \\
\text { imaginary } \\
\text { frequencie } \\
\text { s }\end{array}$ \\
\hline \multirow{3}{*}{$12 a$} & $\mathrm{C}^{4}$ endo & $\begin{array}{c}\text { Twisted } \\
\mathrm{C}^{2} \text { endo }-\mathrm{C}^{3} \text { exo }\end{array}$ & -767.1661 & 129.577 & -766.9596 & 0.8 & 12 & 0 \\
\hline & $\begin{array}{c}\mathrm{C}^{4} \text { endo (with } \\
\text { transannular HB) }\end{array}$ & $\begin{array}{c}\mathrm{C}^{4} \text { endo (with } \\
\text { transannular } \mathrm{HB} \text { ) }\end{array}$ & -767.1675 & 129.734 & -766.9607 & 0.1 & 40 & 0 \\
\hline & $\mathrm{C}^{4}$ exo & $\mathrm{C}^{4}$ exo & -767.1671 & 129.393 & \begin{tabular}{|l|}
-766.9609 \\
\end{tabular} & 0.0 & 47 & $1^{b}$ \\
\hline \multirow[b]{2}{*}{$12 b$} & $\mathrm{C}^{4}$ endo & $\mathrm{C}^{4}$ endo & -767.1684 & 129.545 & -766.9619 & 0.0 & 92 & 0 \\
\hline & $C^{4}$ exo & $\begin{array}{c}\text { Twisted } \\
\mathrm{C}^{3} \text { endo- } \mathrm{C}^{4} \text { exo }\end{array}$ & $\mid-767.1661$ & 129.507 & -766.9597 & 1.4 & 8 & 0 \\
\hline \multirow{3}{*}{$12 \mathrm{c}$} & $\mathrm{C}^{4}$ endo & $\mathrm{C}^{4}$ endo & \begin{tabular}{|l|}
-767.1674 \\
\end{tabular} & 129.322 & \begin{tabular}{|l|}
-766.9613 \\
\end{tabular} & 0.0 & 78 & 0 \\
\hline & $\begin{array}{c}\mathrm{C}^{4} \text { endo (with } \\
\text { transannular } \mathrm{HB} \text { ) }\end{array}$ & $\begin{array}{c}\mathrm{C}^{4} \text { endo (with } \\
\text { transannular } H B \text { ) }\end{array}$ & -767.1665 & 129.513 & $\mid-766.9601$ & 0.8 & 21 & 0 \\
\hline & $C^{4}$ exo & $\begin{array}{c}\text { Twisted } \\
\mathrm{C}^{3} \text { endo- } \mathrm{C}^{4} \text { exo }\end{array}$ & $\mid-767.1631$ & 129.341 & -766.9570 & 2.7 & 1 & 0 \\
\hline \multirow{2}{*}{$12 d$} & $\mathrm{C}^{4}$ endo & $\mathrm{C}^{3}$ exo & -767.1659 & 129.456 & -766.9596 & 0.0 & 60 & 0 \\
\hline & $\mathrm{C}^{4}$ exo & $\mathrm{C}^{5}$ endo & -767.1656 & 129.527 & \begin{tabular}{|l|}
-766.9592 \\
\end{tabular} & 0.2 & 40 & 0 \\
\hline \multirow{2}{*}{$\begin{array}{c}\text { Ac-Hyp- } \\
\text { OMe }\end{array}$} & $\mathrm{C}^{4}$ endo & $\begin{array}{c}\text { Twisted } \\
\mathrm{C}^{3} \text { exo- }-\mathrm{C}^{4} \text { endo }\end{array}$ & -667.9741 & 134.507 & -667.7597 & 0.4 & 35 & 0 \\
\hline & $C^{4}$ exo & $\mathrm{C}^{4}$ exo & -667.9746 & 134.481 & -667.7603 & 0.0 & 65 & 0 \\
\hline \multirow{3}{*}{$\begin{array}{c}\text { Ac-Hyp- } \\
\text { OMe }\end{array}$} & $\mathrm{C}^{4}$ endo & $\mathrm{C}^{3}$ exo & -667.9751 & 134.400 & -667.7609 & 0.0 & 54 & 0 \\
\hline & $\begin{array}{c}\mathrm{C}^{4} \text { endo (with } \\
\text { transannular } H B \text { ) }\end{array}$ & $\begin{array}{c}\mathrm{C}^{4} \text { endo (with } \\
\text { transannular } H B \text { ) }\end{array}$ & -667.9754 & 134.735 & -667.7607 & 0.1 & 43 & 0 \\
\hline & $\mathrm{C}^{4}$ exo & $\mathrm{C}^{4}$ exo & -667.9723 & 134.417 & -667.7581 & 1.8 & 3 & 0 \\
\hline \multirow{2}{*}{$\begin{array}{c}\text { Ac-3-Fpro- } \\
\text { OMe } \\
\end{array}$} & $\mathrm{C}^{4}$ endo & $\mathrm{C}^{4}$ endo & -691.9858 & 126.435 & -691.7843 & 0.0 & 98 & $1^{c}$ \\
\hline & $\mathrm{C}^{4}$ exo & $\mathrm{C}^{4}$ exo & -691.9820 & 126.358 & -691.7807 & 2.3 & 2 & 0 \\
\hline \multirow{2}{*}{\begin{tabular}{|c|} 
Ac-3-fpro- \\
OMe \\
\end{tabular}} & $\mathrm{C}^{4}$ endo & $\mathrm{C}^{3}$ exo & -691.9839 & 126.518 & -691.7822 & 0.4 & 33 & 0 \\
\hline & $\mathrm{C}^{4}$ exo & $\mathrm{C}^{5}$ endo & -691.9845 & 126.485 & -691.7829 & 0.0 & 67 & 0 \\
\hline \multirow{2}{*}{$\begin{array}{c}\text { Ac-Pro- } \\
\text { OMe }\end{array}$} & $\mathrm{C}^{4}$ endo & $\mathrm{C}^{4}$ endo & -592.7893 & 131.508 & -592.5797 & 0.0 & 75 & 0 \\
\hline & $\mathrm{C}^{4}$ exo & $C^{4}$ exo & -592.7880 & 131.365 & -592.5787 & 0.6 & 25 & $1^{d}$ \\
\hline
\end{tabular}

${ }^{a}$ Boltzmann population obtained from relative $\Delta E$.

${ }^{b}$ Frequency $=-4.9 \mathrm{~cm}^{-1}$

${ }^{c}$ Frequency $=-9.9 \mathrm{~cm}^{-1}$

${ }^{d}$ Frequency $=-9.5 \mathrm{~cm}^{-1}$ 
Supplementary Table 3. Pucker preferences, conformational analysis, second-order perturbation energies, and Kenny electrostatic potential for F-Hyps 12a-d, Ac-Hyp-OMe, Ac-hyp-OMe, Ac-3-Fpro-OMe, Ac-3-fpro-OMe and Ac-Pro-OMe. DFT calculations were carried out at the MN15-L/aug-cc-pVTZ(-F) level of theory in water solvation continuum.

\begin{tabular}{|c|c|c|c|c|c|c|c|c|c|c|c|c|c|c|c|}
\hline \multirow{3}{*}{ Compound } & \multirow{3}{*}{$\begin{array}{c}\text { Ratio } \\
\text { trans:cis }\end{array}$} & \multirow{3}{*}{$\begin{array}{c}J_{\mathrm{H} 2-\mathrm{H}} \\
(\mathrm{Hz})\end{array}$} & \multirow{3}{*}{$\begin{array}{l}\text { Calculated } \\
\bar{J}_{\mathrm{H} 2-\mathrm{H} 3}(\mathrm{~Hz})\end{array}$} & \multirow{3}{*}{$\begin{array}{c}\text { Canonical } \\
\text { starting pucker }\end{array}$} & \multirow{3}{*}{$\begin{array}{l}\text { Optimized } \\
\text { pucker }\end{array}$} & \multirow{3}{*}{$\begin{array}{c}\text { Fraction of } \\
\text { conformer } \\
(\%)^{b}\end{array}$} & \multicolumn{3}{|c|}{ Dihedral angle (ㅇ) } & \multicolumn{4}{|c|}{$E_{n \rightarrow \pi^{*}}^{(2)}(\mathrm{kcal} / \mathrm{mol})$} & \multicolumn{2}{|c|}{$V_{\alpha}(r)(\text { a.u. })^{e}$} \\
\hline & & & & & & & \multirow[b]{2}{*}{$C^{5}-N-C^{2}-C^{3}$} & \multirow[b]{2}{*}{$N-C^{5}-C^{4}-O$} & \multirow[b]{2}{*}{$N-C^{2}-C^{3}-F$} & \multicolumn{2}{|c|}{$\mathrm{O} \rightarrow \mathrm{C}=\mathrm{O}^{c}$} & \multicolumn{2}{|c|}{$\mathrm{F} \rightarrow \mathrm{C}=\mathrm{O}^{d}$} & \multirow[b]{2}{*}{$\begin{array}{c}\text { Per } \\
\text { conformer }\end{array}$} & \multirow[b]{2}{*}{ Weighted } \\
\hline & & & & & & & & & & $\begin{array}{c}\text { Per } \\
\text { conformer }\end{array}$ & Weighted & $\begin{array}{c}\text { Per } \\
\text { conformer }\end{array}$ & Weighted & & \\
\hline \multirow{3}{*}{$12 a$} & \multirow{3}{*}{$88: 12$} & \multirow{3}{*}{4.6} & \multirow{3}{*}{5.7} & $C^{4}$ endo & $\begin{array}{c}\text { Twisted } \\
\mathrm{C}^{2} \text { endo- } \mathrm{C}^{3} \text { exo }\end{array}$ & 12 & -36.5 & -103 & 165 & 0.0 & \multirow{3}{*}{0.8} & 0.0 & \multirow{3}{*}{1.1} & 0.357 & \multirow{3}{*}{0.339} \\
\hline & & & & $\begin{array}{c}\mathrm{C}^{4} \text { endo (with } \\
\text { transannular } \mathrm{HB} \text { ) }\end{array}$ & $\begin{array}{l}\mathrm{C}^{4} \text { endo (with } \\
\text { transannular } \mathrm{HB} \text { ) }\end{array}$ & 40 & -2.7 & -80 & 151 & 1.4 & & 1.0 & & 0.282 & \\
\hline & & & & $C^{4}$ exo & $\mathrm{C}^{4}$ exo & 47 & 2.4 & -162 & 86 & 0.5 & & 1.4 & & 0.383 & \\
\hline \multirow[b]{2}{*}{$12 b$} & \multirow[b]{2}{*}{$84: 16$} & \multirow[b]{2}{*}{2.3} & \multirow[b]{2}{*}{1.2} & $\mathrm{C}^{4}$ endo & $\mathrm{C}^{4}$ endo & 92 & -9.4 & 161 & -84 & 0.7 & \multirow[b]{2}{*}{1.0} & & & 0.380 & \\
\hline & & & & $C^{4}$ exo & $\begin{array}{c}\text { Twisted } \\
\mathrm{C}^{3} \text { endo- } \mathrm{C}^{4} \text { exo }\end{array}$ & 8 & 14.1 & 82 & -157 & 3.9 & & - & - & 0.360 & 0.378 \\
\hline & & & & $\mathrm{C}^{4}$ endo & $\mathrm{C}^{4}$ endo & 78 & -7.1 & -87 & -93 & 0.4 & & & & 0.372 & \\
\hline $12 c$ & $71: 29$ & $<0.5$ & 0.1 & $\begin{array}{c}\mathrm{C}^{4} \text { endo (with } \\
\text { transannular } \mathrm{HB} \text { ) }\end{array}$ & $\begin{array}{c}\mathrm{C}^{4} \text { endo (with } \\
\text { transannular } \mathrm{HB} \text { ) }\end{array}$ & 21 & 12.0 & -77 & -105 & 2.6 & 0.9 & - & - & 0.300 & 0.357 \\
\hline & & & & $c^{4}$ exo & $\begin{array}{c}\text { Twisted } \\
\mathrm{C}^{3} \text { endo- } \mathrm{C}^{4} \text { exo }\end{array}$ & 1 & 16.6 & -160 & -162 & 4.1 & & & & 0.373 & \\
\hline & & & & $\mathrm{C}^{4}$ endo & $c^{3}$ exo & 60 & -25.2 & 152 & 166 & 0.3 & & 0.2 & & 0.369 & \\
\hline $12 \mathrm{~d}$ & $87: 13$ & 5.0 & 7.0 & $\mathrm{C}^{4}$ exo & $c^{5}$ endo & 40 & -20.2 & 81 & 114 & 0.5 & 0.4 & 4.1 & 1.7 & 0.382 & 0.375 \\
\hline Ac-Нур-ОМе & $86: 1$ & - & - & $C^{4}$ endo & $\begin{array}{c}\text { Twisted } \\
\mathrm{C}^{3} \text { exo- } \mathrm{C}^{4} \text { endo }\end{array}$ & 35 & -14.6 & 158 & - & 0.3 & 2.1 & - & - & 0.372 & 0.371 \\
\hline & & & & $C^{4}$ exo & $\mathrm{C}^{4}$ exo & 65 & 11.3 & 85 & & 3.1 & & & & 0.370 & \\
\hline & & & & $\mathrm{C}^{4}$ endo & $\mathrm{C}^{3}$ exo & 54 & -24.0 & -96 & & 0.0 & & & & 0.367 & \\
\hline Ac-hyp-OMe & $71: 29\left(\right.$ ref. $\left.^{1}\right)$ & - & - & $\begin{array}{c}\mathrm{C}^{4} \text { endo (with } \\
\text { transannular } \mathrm{HB} \text { ) }\end{array}$ & $\begin{array}{l}\mathrm{C}^{4} \text { endo (with } \\
\text { transannular } \mathrm{HB} \text { ) }\end{array}$ & 43 & 4.3 & -81 & - & 2.6 & 1.2 & - & - & 0.268 & 0.324 \\
\hline & & & & $C^{4}$ exo & $C^{4}$ exo & 3 & 9.8 & -160 & & 2.9 & & & & 0.374 & \\
\hline Ac-3-Fpro- & $81 \cdot 19\left(\operatorname{ref}^{1}\right)$ & $<1$ & 0.4 & $\mathrm{C}^{4}$ endo & $\mathrm{C}^{4}$ endo & 98 & -2.7 & - & -92 & 1.7 & & - & - & - & - \\
\hline $\mathrm{OMe}$ & 81:19(ret. ) & $<1$ & 0.4 & $\mathrm{C}^{4}$ exo & $C^{4} e x O$ & 2 & 11.6 & - & -157 & 3.3 & 1.1 & - & - & - & - \\
\hline Ac-3-fpro- & $90: 10\left(\right.$ ref $\left.^{2}\right)$ & - & 6.5 & $\mathrm{C}^{4}$ endo & $c^{3}$ exo & 33 & -21.1 & - & 161 & 0.4 & 0.4 & 0.5 & 22 & - & - \\
\hline $\mathrm{OMe}$ & 90:10 (ret. ) & - & 0.5 & $c^{4}$ exo & $C^{5}$ endo & 67 & -18.3 & - & 108 & 0.4 & 0.4 & 3.1 & 2.2 & - & - \\
\hline Ac-Pro-OMe & $82: 18$ (ref. $\left.^{2}\right)$ & - & - & $\mathrm{C}^{4}$ endo & $\mathrm{C}^{4}$ endo & 75 & -11.2 & - & - & 0.5 & 0.8 & - & - & - & - \\
\hline Ac-Pro-uive & 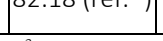 & 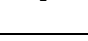 & 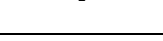 & $\mathrm{C}^{4}$ exo & $\mathrm{C}^{4}$ exo & 25 & 6.0 & - & - & 1.6 & & - & - & - & - \\
\hline
\end{tabular}

${ }^{a}$ Measured as the ratio of proton-decoupled ${ }^{19} \mathrm{~F}-\mathrm{NMR}$ signals in $\mathrm{D}_{2} \mathrm{O}$

Boltzmann population obtained from relative $\triangle E$ (Supp. Table 2).

${ }^{c}$ Charge transfer from acetyl O lone pair to $\pi^{*}$ orbital in the carbonyl in $\mathrm{C}^{2}$

${ }^{d}$ Charge transfer from $\mathrm{F}$ lone pair to $\pi^{*}$ orbital in the carbonyl in $\mathrm{C}^{2}$.

${ }^{e}$ The Kenny electrostatic potential correlates with $\mathrm{H}$-bond donor capacity ${ }^{3}$ 
Supplementary Table 4. Summary of geometric, topological, and energetic analysis of transannular H-bond conformers in 12a,c and Ac-hyp-OMe. DFT calculations were carried out at the MN15-L/aug-cc-pVTZ(-F) level of theory in water solvation continuum.

\begin{tabular}{|c|c|c|c|c|}
\hline Compound & $\begin{array}{c}\text { Fraction of } \\
\text { transannular } \\
\text { H-bond (\%) }\end{array}$ & $d_{\mathrm{O} \ldots \mathrm{HO}}(\AA)$ & $\rho_{\mathrm{BCP}}(e)^{a}$ & $\begin{array}{c}E_{n_{\mathrm{O}} \rightarrow \sigma_{\mathrm{OH}}^{*}}^{(2)} \\
(\mathrm{kcal} / \mathrm{mol})^{b}\end{array}$ \\
\hline $12 \mathrm{a}$ & 40 & 2.07 & 0.0188 & 3.1 \\
\hline $12 \mathrm{c}$ & 21 & 2.28 & 0.0124 & 1.4 \\
\hline Ac-hyp-OMe & 43 & 2.06 & 0.0192 & 4.0 \\
\hline
\end{tabular}

${ }^{a}$ Electron density at the BCP of the transannular $\mathrm{H}$-bond.

${ }^{b}$ Charge transfer from $\mathrm{O}$ lone pair to $\sigma^{*}$ orbital in the $\mathrm{OH}$.

Supplementary Table 5. ITC results of binary and ternary complex formation for PROTACs (values reported are the mean $\pm S E M, N . R .=$ number of replicates). $\alpha=$ cooperativity

\begin{tabular}{|c|c|c|c|c|c|c|c|c|c|}
\hline Entry & $\begin{array}{l}\text { Species in } \\
\text { the cell }\end{array}$ & $\begin{array}{l}\text { Protein in } \\
\text { the syringe }\end{array}$ & $\begin{array}{c}K_{\mathrm{d}} \\
(\mathrm{nM})\end{array}$ & $\begin{array}{c}\Delta H \\
(\mathrm{kcal} / \mathrm{mol})\end{array}$ & $\begin{array}{c}-\mathrm{T} \Delta S \\
(\mathrm{kcal} / \mathrm{mol})\end{array}$ & $\begin{array}{c}\Delta G \\
(\mathrm{kcal} / \mathrm{mol})\end{array}$ & $\alpha$ & $\begin{array}{c}\Delta \Delta G \\
(\mathrm{kcal} / \mathrm{mol})\end{array}$ & N.R. \\
\hline 1 & \multirow{3}{*}{$15 a$} & $\operatorname{Brd} 2^{\mathrm{BD} 1}$ & $97 \pm 0.6$ & $-13.7 \pm 0.3$ & $4.2 \pm 0.3$ & $-9.57 \pm 0.01$ & - & - & 2 \\
\hline 2 & & $\mathrm{Brd} 4^{\mathrm{BD} 2}$ & $28 \pm 2$ & $-10.4 \pm 0.8$ & $0.1 \pm 0.6$ & $\begin{array}{c}-10.30 \pm \\
0.02\end{array}$ & - & - & 2 \\
\hline 3 & & VBC & $249 \pm 6$ & $-7.9 \pm 0.3$ & $-1.1 \pm 0.2$ & $-9.01 \pm 0.02$ & - & - & 3 \\
\hline 4 & $\mathbf{1 5} \mathbf{a}: \operatorname{Brd} 2^{\mathrm{BD} 1}$ & \multirow{2}{*}{ VBC } & $64 \pm 3$ & $\begin{array}{c}-9.17 \pm \\
0.04\end{array}$ & $-0.6 \pm 0.6$ & $-9.85 \pm 0.05$ & 3.9 & $\begin{array}{c}-0.85 \pm \\
0.05\end{array}$ & 2 \\
\hline 5 & 15a:Brd $4^{\mathrm{BD} 2}$ & & $26 \pm 5$ & $-10.9 \pm 0.7$ & $-0.6 \pm 0.4$ & $\begin{array}{c}-10.13 \pm \\
0.08\end{array}$ & 9.5 & $\begin{array}{l}-1.12 \\
\pm 0.08\end{array}$ & 2 \\
\hline 6 & \multirow{3}{*}{$15 b$} & $\mathrm{Brd} 2^{\mathrm{BD} 1}$ & $111 \pm 6$ & $-14.1 \pm 0.6$ & $4.9 \pm 0.6$ & $-9.49 \pm 0.03$ & - & - & 2 \\
\hline 7 & & $\mathrm{Brd} 4^{\mathrm{BD} 2}$ & $32 \pm 3$ & $-9.3 \pm 0.4$ & $-0.9 \pm 0.4$ & $-10.3 \pm 0.1$ & - & - & 2 \\
\hline 8 & & VBC & $603 \pm 74$ & $-5.0 \pm 0.2$ & $-3.7 \pm 0.2$ & $-8.49 \pm 0.07$ & - & - & 2 \\
\hline 9 & 15b:Brd2 $2^{\mathrm{BD} 1}$ & \multirow{2}{*}{ VBC } & $500 \pm 96$ & $-5.1 \pm 0.2$ & $-3.6 \pm 0.3$ & $-8.6 \pm 0.1$ & 1.2 & $-0.1 \pm 0.1$ & 2 \\
\hline 10 & 15b:Brd4 ${ }^{\mathrm{BD} 2}$ & & $42 \pm 6$ & $\begin{array}{c}-7.75 \pm \\
0.04\end{array}$ & $\begin{array}{c}-2.34 \pm \\
0.04\end{array}$ & $\begin{array}{c}-10.01 \pm \\
0.01\end{array}$ & 14.5 & $-1.6 \pm 0.1$ & 2 \\
\hline
\end{tabular}


Supplementary Table 6. X-ray crystallography: data collection \& refinement statistics. Values in parentheses correspond to the highest resolution shell.

\begin{tabular}{|c|c|c|c|}
\hline Dataset & Peptide 13a:VBC & Ligand 14a:VBC & Ligand 14b:VBC \\
\hline Synchrotron & Diamond Light Source & Diamond Light Source & Diamond Light Source \\
\hline Beamline & I03 & I04-1 & I04 \\
\hline Wavelength $(\AA)$ & 0.9763 & 0.9282 & 0.9795 \\
\hline \multicolumn{4}{|l|}{ Processing statistics } \\
\hline Space group & $P 4_{3} 2{ }_{1} 2$ & $P 4_{1} 22$ & $P 4_{1} 22$ \\
\hline \multicolumn{4}{|l|}{ Unit cell parameters } \\
\hline$a, b(\AA)$ & 59.3 & 94.1 & 93.6 \\
\hline$c(\AA)$ & 245.3 & 368.0 & 364.5 \\
\hline Resolution limits $(\AA)$ & $49.07-1.83(1.87-1.83)$ & $49.4-2.7(2.79-2.70)$ & $49.0-2.3(2.35-2.30)$ \\
\hline Total reflections & $679485(36789)$ & $405413(35686)$ & $644172(39520)$ \\
\hline Unique reflections & $39987(2427)$ & $46704(4440)$ & $73389(4441)$ \\
\hline Completeness (\%) & $100.0(99.9)$ & $99.9(99.8)$ & $100(100)$ \\
\hline Multiplicity & $17.0(15.2)$ & $8.7(8.0)$ & $8.8(8.9)$ \\
\hline$R_{\text {merge }}(\%)$ & $9.5(108.3)$ & $14.9(76.0)$ & $8.5(89.8)$ \\
\hline$I / \sigma(I)$ & $17.8(2.5)$ & $8.2(2.4)$ & $14.5(2.4)$ \\
\hline $\mathrm{CC}_{1 / 2}(\%)$ & $99.9(87.0)$ & $99.7(81.5)$ & $99.9(82.0)$ \\
\hline Mosaicity $\left({ }^{\circ}\right)$ & 0.13 & 0.20 & 0.05 \\
\hline \multicolumn{4}{|l|}{ Refinement statistics } \\
\hline Resolution limits $(\AA)$ & $61.3-1.83(1.87-1.83)$ & $49.4-2.7(2.77-2.70)$ & $49.0-2.3(2.36-2.30)$ \\
\hline$R_{\text {work }}(\%)$ & $18.3(21.5)$ & $19.9(29.7)$ & $19.5(24.9)$ \\
\hline$R_{\text {free }}(\%)$ & $21.2(26.1)$ & $25.8(36.4)$ & $24.5(29.0)$ \\
\hline Model atoms & 3138 & 11004 & 11439 \\
\hline Protein $B$ factor $\left(\AA^{2}\right)$ & 31.1 & 53.1 & 55.8 \\
\hline Peptide/Ligand $B$ factor $\left(\AA^{2}\right)$ & 44.6 & 35.8 & 39.5 \\
\hline r.m.s.d. bonds $(\AA)$ & 0.010 & 0.007 & 0.008 \\
\hline r.m.s.d. angles $\left({ }^{\circ}\right)$ & 1.43 & 1.18 & 1.31 \\
\hline \multicolumn{4}{|l|}{ Ramanchandran plot } \\
\hline Favoured (\%) & 97.1 & 96.8 & 96.9 \\
\hline Allowed (\%) & 2.9 & 3.1 & 3.0 \\
\hline Disallowed (\%) & 0.0 & 0.1 & 0.1 \\
\hline PDB code & 6GFX & 6GFY & 6GFZ \\
\hline
\end{tabular}




\section{Supplementary figures}

A)

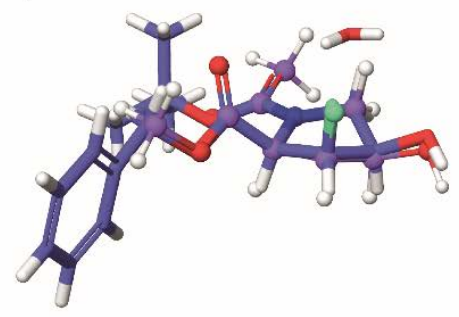

B)

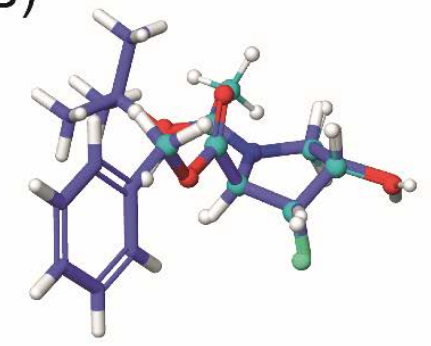

C)

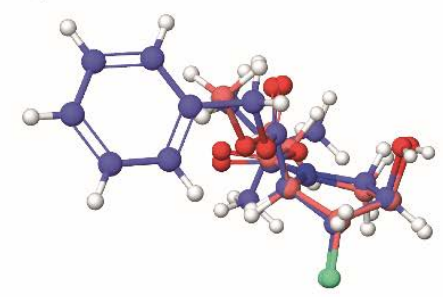

Supplementary Figure 1. Superposition of global energetic minima found for F-Hyps 12a-c and small-molecule $X$-ray crystal structure of related compounds $4 a-c$ (in blue sticks). A) $12 a$ (purple) and $4 a, B) 12 b$ (cyan) and $4 b$, and C) $12 c$ (orange) and $4 c$.

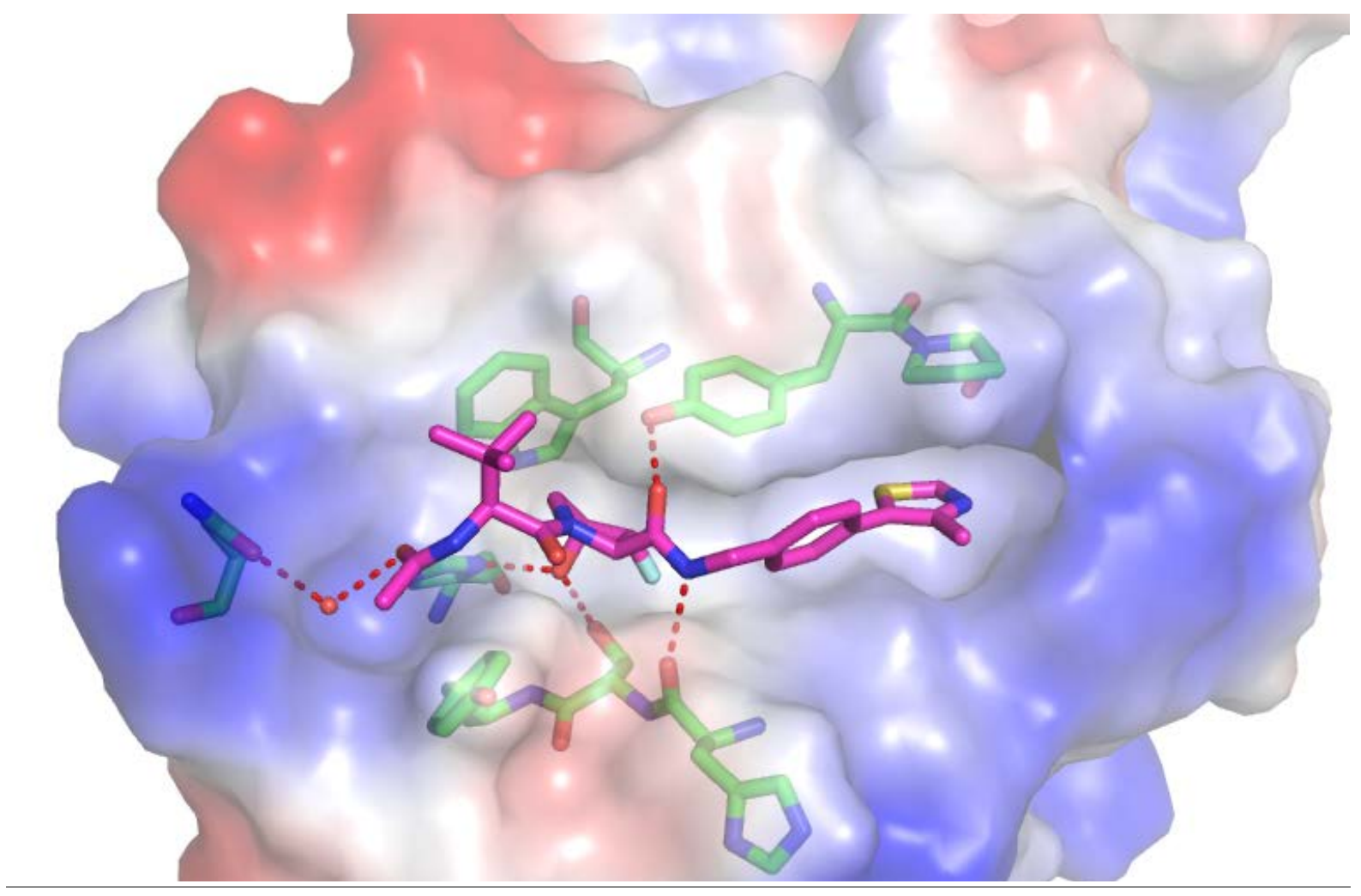

Supplementary Figure 2. Crystal structure of ligand 14 a in complex with VBC. Key H-bond interactions are shown in red. 


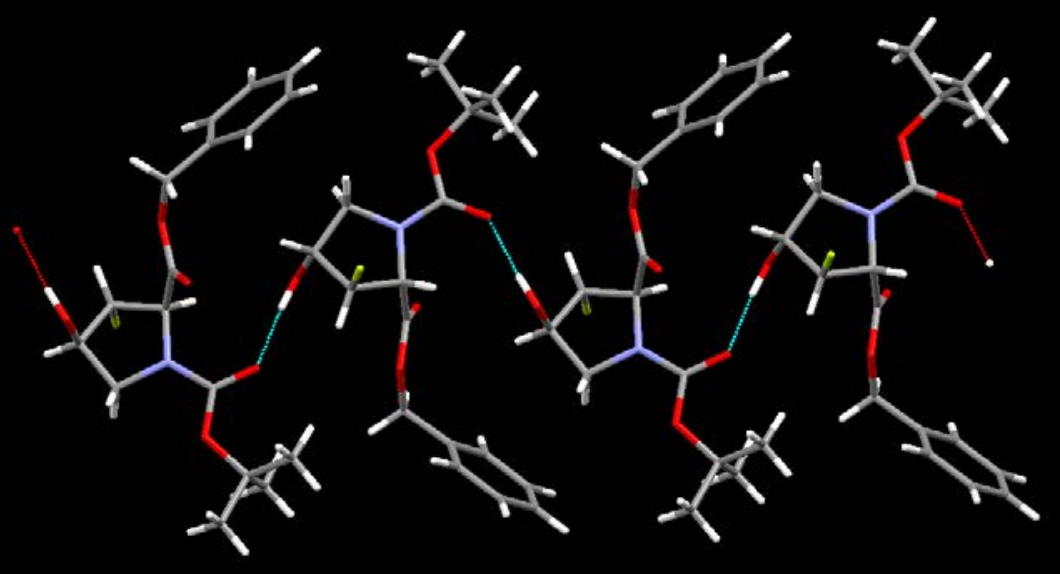

Supplementary Figure 3. Part of an [010] hydrogen-bonded chain in the small-molecule Xray crystal structure of $\mathbf{4 c}$.

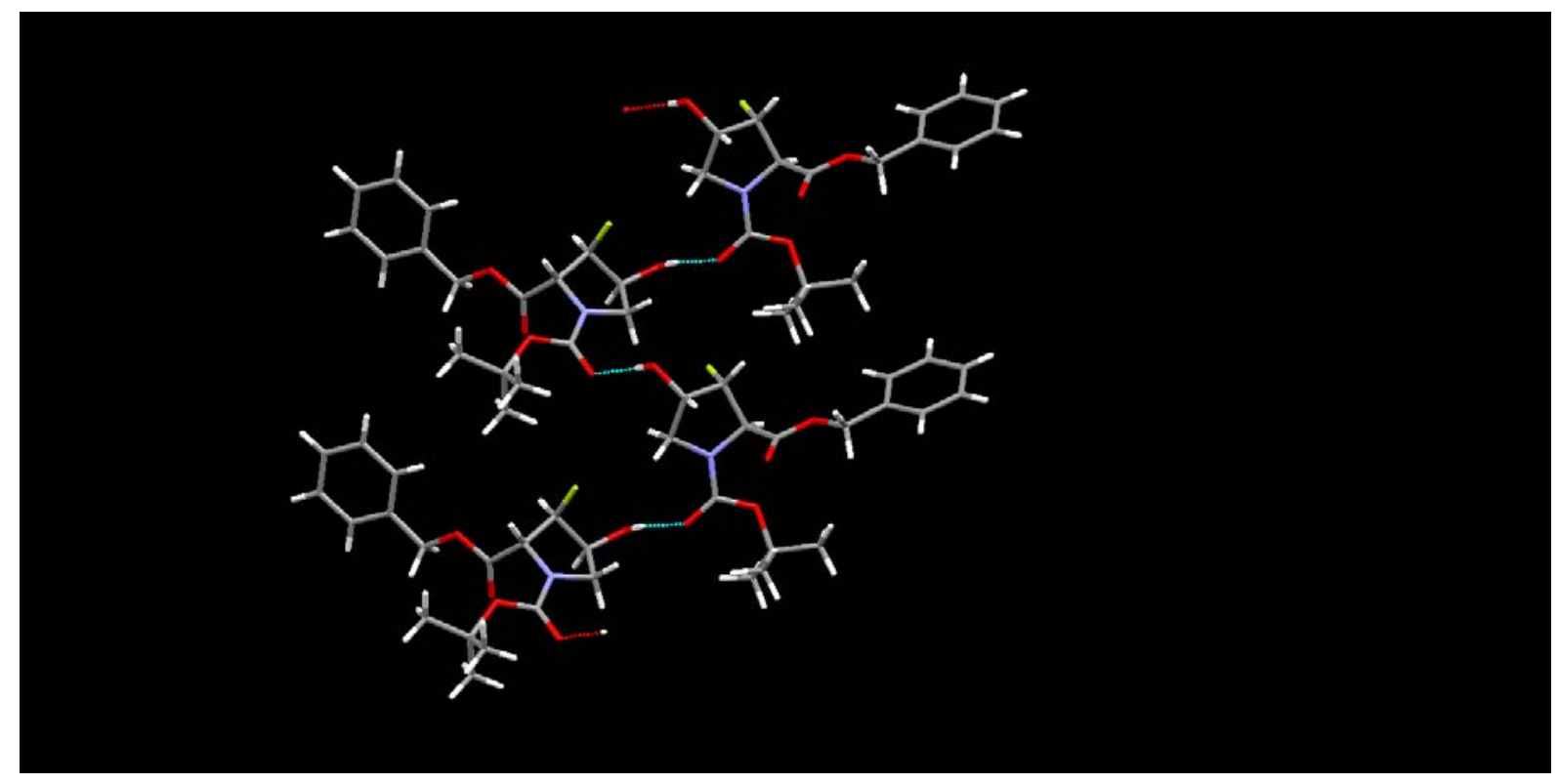

Supplementary Figure 4. Part of a [100] hydrogen-bonded chain in the small-molecule X-ray crystal structure of $\mathbf{4 b}$. 


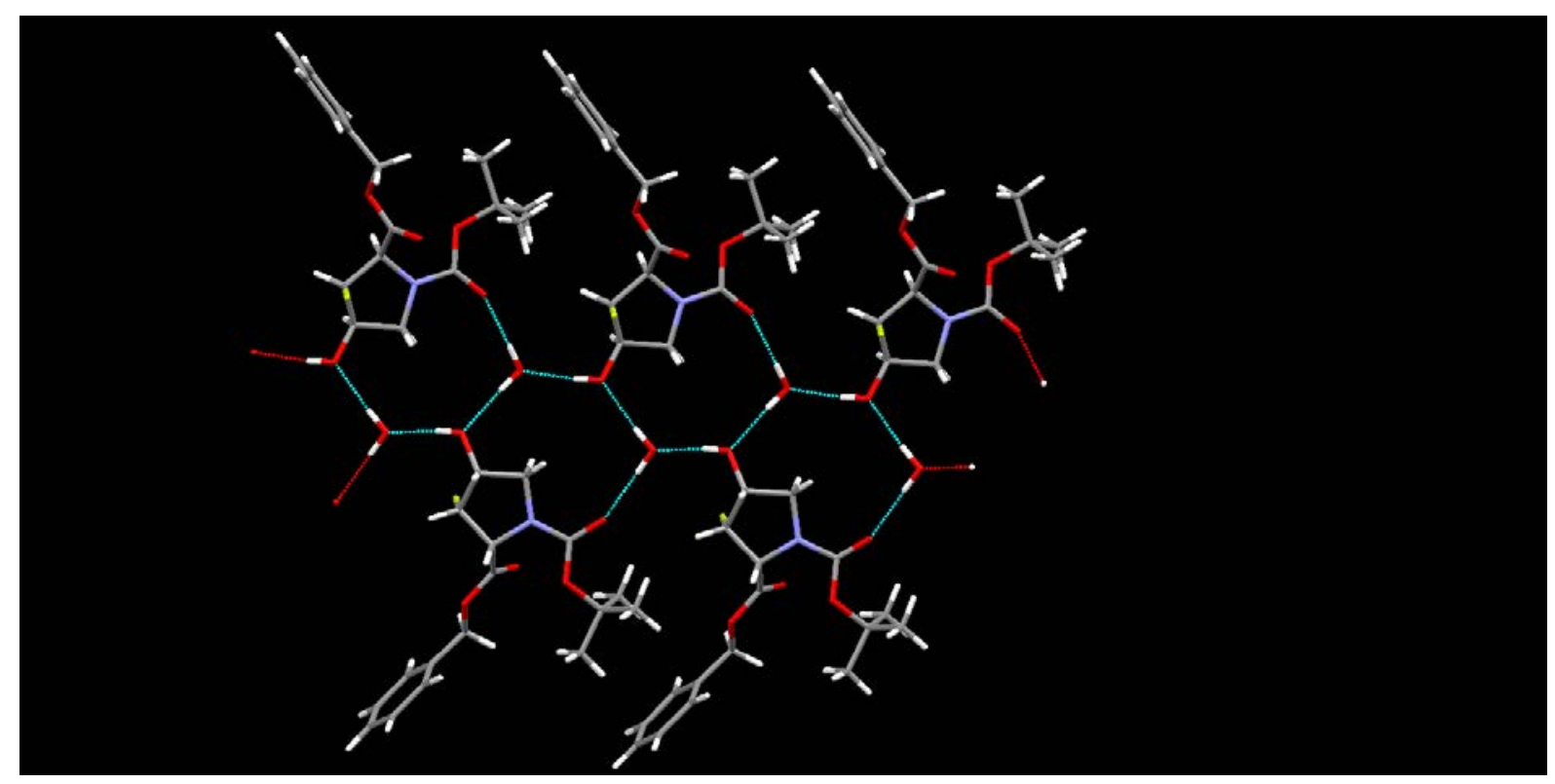

Supplementary Figure 5. Part of an [010] hydrogen-bonded chain in the small-molecule Xray crystal structure of 4 a involving both organic and water molecules. 


\section{Chemistry - Materials and Methods}

All chemicals, unless otherwise stated were commercially available and used without further purification. Solvents were anhydrous and reactions preformed under positive pressure of nitrogen or argon. Enantiopure (+)-JQ-1 was purchased from Medchemexpress LLC, Princeton, USA. Flash column chromatography (FCC) was performed using a Teledyne Isco Combiflash Rf or Rf200i. As prepacked columns RediSep Rf Normal Phase Disposable Columns were used.

NMR spectra were recorded on a Bruker 500 Ultrashield or a Bruker Ascend 400. Chemical shifts are quoted in ppm and referenced to the residual solvent signals: ${ }^{1} \mathrm{H} \delta=7.26\left(\mathrm{CDCl}_{3}\right),{ }^{13} \mathrm{C} \delta=77.16$ $\left(\mathrm{CDCl}_{3}\right),{ }^{1} \mathrm{H} \delta=3.31$ (MeOD), ${ }^{13} \mathrm{C} \delta=49.15(\mathrm{MeOD}),{ }^{1} \mathrm{H} \delta=4.79\left(\mathrm{D}_{2} \mathrm{O}\right)$. Signal splitting patterns are described as singlet (s), doublet (d), triplet (t), quartet (q), multiplet (m), broad (br) or a combination thereof. Coupling constants $(J)$ are measured in Hz. Symbol "*” labels the signals of the minor rotamer when clearly distinguishable from the major one.

Low resolution MS and analytical HPLC traces were recorded on an Agilent Technologies 1200 series HPLC connected to an Agilent Technologies 6130 quadrupole LC/MS, connected to an Agilent diode array detector. The column used was a Waters XBridge column $(50 \mathrm{~mm} \times 2.1 \mathrm{~mm}, 3.5 \mu \mathrm{m}$ particle size) and the compounds were eluted with a gradient of 5-95\% acetonitrile/water $+0.1 \%$ formic acid over $3 \mathrm{~min}$ (METHOD 1) or over $7 \mathrm{~min}$ (METHOD 2).

Preparative HPLC was performed on a Gilson Preparative HPLC System with a Waters X-Bridge C18 column (100 mm x $19 \mathrm{~mm} ; 5 \mu \mathrm{m}$ particle size) and a gradient of $5 \%$ to $95 \%$ acetonitrile in water over $10 \mathrm{~min}$, flow $25 \mathrm{~mL} / \mathrm{min}$, with $0.1 \%$ formic acid in the aqueous phase.

Abbreviations used: $\mathrm{ACN}$ for acetonitrile, DCM for dichloromethane, EtOAc for ethyl acetate, $\mathrm{Et}_{2} \mathrm{O}$ for diethyl ether, DMSO for dimethyl sulfoxide, DIPEA for N,N-diisopropylethylamine, $\mathrm{MeOH}$ for methanol, TEA for triethylamine, DMF for N,N-dimethylformamide, HATU for 1[bis(dimethylamino)methylene]-1H-1,2,3-triazolo[4,5-b]pyridinium 3-oxid hexafluorophosphate, HOAT for 1-hydroxy-7-azabenzotriazole, TMSOTf for trimethylsilyltriflate, TFA for trifluoroacetic acid. 


\section{2-Benzyl 1-(tert-butyl) (2R)-3-fluoro-4-oxopyrrolidine-1,2-dicarboxylate (3)}

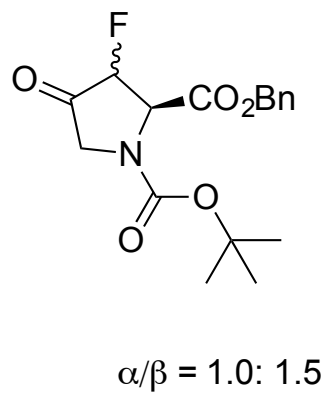

A solution of 2-benzyl 1-(tert-butyl) (S)-4-oxopyrrolidine-1,2-dicarboxylate (1) (1.255 g, 3.929 $\mathrm{mmol})$ in THF ( $5 \mathrm{~mL}$ ) was added dropwise to a stirred solution of LHMDS (1M in THF, $4.35 \mathrm{~mL})$ in THF $(4.35 \mathrm{~mL})$ at $-78^{\circ} \mathrm{C}$. The mixture was stirred at $-78^{\circ} \mathrm{C}$ for $2 \mathrm{~h}$, then TMSCl $(1.25 \mathrm{~mL}, 10 \mathrm{mmol})$ was added dropwise. After 30 min of continued stirring at $-78{ }^{\circ} \mathrm{C}$, the cooling bath was removed and the reaction mixture was allowed to warm to r.t. and stirred for additional $3 \mathrm{~h}$. The reaction mixture was then concentrated under vacuum to small volume, pentane $(50 \mathrm{~mL})$ was added and the mixture was poured into a saturated solution of $\mathrm{NaHCO}_{3}(100 \mathrm{~mL})$. The mixture was vigorously shaken and the organic layer was separated, washed with brine, then dried over anhydrous $\mathrm{MgSO}_{4}$. The volatiles were removed under reduced pressure to obtain a light yellow oil corresponding to the trimethyl silyl enol ether (2) which was dissolved in acetonitrile $(60 \mathrm{~mL})$ and cooled to $0{ }^{\circ} \mathrm{C}$. Selectfluor $(2.040 \mathrm{~g}$, $5.750 \mathrm{mmol}$ ) was added in one portion and the mixture was carefully allowed to reach $10^{\circ} \mathrm{C}$ overnight. TLC analysis (EtOAc/Heptane 3:7) showed complete conversion of the starting material. A saturated solution of $\mathrm{NH}_{4} \mathrm{Cl}$ was added $(30 \mathrm{ML})$, the resulting mixture was vigorously shaken and the organic layer was separated. The aqueous layer was extracted with EtOAc $(2 \times 30 \mathrm{~mL})$ and the organic phase was washed with brine, then dried over $\mathrm{MgSO}_{4}$. Volatiles were removed under reduced pressure to obtain a yellow oil which was quickly passed through a short silica column eluted with a mixture of EtOAc/Heptane 4:6 to obtain the desired crude fluoroketone (3) (662 mg 50\% yield) as a mixture of diasteroisomers. This crude mixture proved to be unstable in solution and therefore was used immediately in the next step. Alternatively, 2-Benzyl 1-(tert-butyl) (S)-4-((trimethylsilyl)oxy)2,5-dihydro-1H-pyrrole-1,2-dicarboxylate and Selectfluor were dissolved in anhydrous acetonitrile ( $0.067 \mathrm{M}$ and $0.1 \mathrm{M}$ respectively) and stored under inert atmosphere. The solutions of silyl enol ether and Selectfluor were pumped at a rate of $0.77 \mathrm{~mL} / \mathrm{min}$ in a $10 \mathrm{~mL}$ flow reactor (residence time $6.5 \mathrm{~min}$ ) heated at $50 \mathrm{C}$. The crude product treated with a saturated solution of $\mathrm{NH}_{4} \mathrm{Cl}$ and extracted with ethyl acetate and the organic phase was dried over $\mathrm{MgSO}_{4}$. Solvents were evaporated to obtain the crude product which can be further purified by column chromatography on silica accordingly to the conditions described for the batch synthesis above. This compound proved to be unstable in solution and therefore was used immediately in the next step. 
${ }^{1} \mathrm{H}-\mathrm{NMR}\left(500 \mathrm{MHz}, \mathrm{CDCl}_{3}\right.$, mixture of diasteroisomers and their cis/trans rotamer,) $\delta: 7.40-7.34$ (m, $5 \mathrm{H}), 5.38-4.81(\mathrm{~m}, 4 \mathrm{H}), 4.12-3.95(\mathrm{~m}, 2 \mathrm{H}), 1.49-1.39(\mathrm{~m}, 9 \mathrm{H}) .{ }^{19} \mathrm{~F}$ NMR $\left(470.6 \mathrm{MHz}, \mathrm{CDCl}_{3}\right) \delta$ : $186.91,-187.57$ ( $\alpha$ isomer) -205.18, -205.92 ( $\beta$ isomer).

Reduction of 2-benzyl 1-(tert-butyl) (2R)-3-fluoro-4-oxopyrrolidine-1,2-dicarboxylate with $\underline{\mathrm{NaBH}}_{4}$ to prepare compounds $4 \mathrm{a}, 4 \mathrm{~b}$, and $4 \mathrm{c}$

Fluoroketone 3 (500 mg, $1.482 \mathrm{mmol})$ was dissolved in a mixture of THF/EtOH 1:1 (10 mL) and cooled to $0{ }^{\circ} \mathrm{C}$. $\mathrm{NaBH}_{4}(56 \mathrm{mg}, 1.482 \mathrm{mmol})$ was added portion-wise and the reaction mixture was stirred for $1 \mathrm{~h}$ at $0 \mathrm{C}$. TLC analysis (EtOAc/Heptane 1:1) showed complete conversion of the starting material. The reaction mixture was concentrated under vacuum, EtOAc was added $(15 \mathrm{~mL})$ and a solution of $\mathrm{NaHSO}_{4}(5 \%)$ was added dropwise until a pH of 3 to 4 was obtained. The acidified mixture was then washed with brine $(10 \mathrm{~mL})$, and the organic phase was dried over anhydrous $\mathrm{MgSO}_{4}$. Volatiles were removed under reduced pressure to obtain yellow oil which was subjected to FCC (EtOAc/Heptane 3:7 to 1:1) to separate the 3 diasteroisomers (described herein in order of elution). Alternatively, the mixture was dissolved in methanol $(8 \mathrm{~mL})$ and separated by preparative HPLC (5-90 \% ACN in $\mathrm{H}_{2} \mathrm{O}$ over $\left.15 \mathrm{~min}\right)$.

\section{2-Benzyl 1-(tert-butyl) $(2 R, 3 R, 4 R)-3$-fluoro-4-hydroxypyrrolidine-1,2-dicarboxylate $(4 c)$}

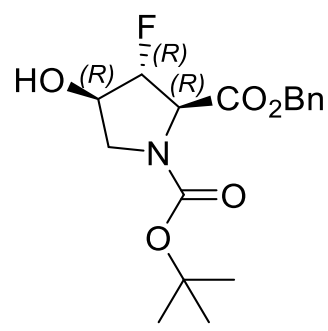

Compound $4 \mathbf{c}$ was obtained as a white solid, $8 \%$ isolated yield (11\% yield by $\left.{ }^{19} \mathrm{~F}-\mathrm{NMR}\right) .{ }^{1} \mathrm{H}-\mathrm{NMR}$ $\left(500 \mathrm{MHz}, \mathrm{CDCl}_{3}\right)$ \&: 7.37-7.36 (m, 5H), 5.33-5.15 (m, 2H), $4.99\left(\mathrm{~d},{ }^{1} J_{\mathrm{H}-\mathrm{F}}=49.1 \mathrm{~Hz}, 1 \mathrm{H}\right), 4.95 *\left(\mathrm{~d},{ }^{1} J\right.$ H-F $=49.0 \mathrm{~Hz}, 1 \mathrm{H}), 4.60 *\left(\mathrm{~d},{ }^{2} J_{\mathrm{H}-\mathrm{F}}=24.8 \mathrm{~Hz}, 1 \mathrm{H}\right), 4.48\left(\mathrm{~d},{ }^{2} J_{\mathrm{H}-\mathrm{F}}=24.8 \mathrm{~Hz}, 1 \mathrm{H}\right), 4.33-4.29(\mathrm{~m}, 1 \mathrm{H})$, 3.74-3.64 (m, 1H), 2.81* (d, $\left.J_{\mathrm{H}-\mathrm{H}}=9.3 \mathrm{~Hz}, 1 \mathrm{H}\right), 2.72\left(\mathrm{~d}, J_{\mathrm{H}-\mathrm{H}}=9.5 \mathrm{~Hz}, 1 \mathrm{H}\right), 1.48 *(\mathrm{~s}, 9 \mathrm{H}), 1.34(\mathrm{~s}$, 9H). ${ }^{19}$ F-NMR (470.6 MHz, $\left.\left.\mathrm{CDCl}_{3}\right) \delta:-179.71^{*},-180.11 .{ }^{13} \mathrm{C} \mathrm{NMR} \mathrm{(125.8} \mathrm{MHz,} \mathrm{CDCl}_{3}\right): 170.5(\mathrm{~d}$, $\left.{ }^{3} J_{\mathrm{C}-\mathrm{F}}=15.9 \mathrm{~Hz}\right), 154.3^{*}, 153.6,134.89^{*}, 134.72,128.83,128.75,128.71,128.64,128.56,128.27$, $96.96\left(\mathrm{~d},{ }^{1} J_{\mathrm{C}-\mathrm{F}} 191.1 \mathrm{~Hz}\right), 95.91 *\left(\mathrm{~d},{ }^{1} J_{\text {C-F }} 191.2 \mathrm{~Hz}\right), 80.98,80.94 *, 73.64 *\left(\mathrm{~d},{ }^{2} J_{\mathrm{C}-\mathrm{F}}=27.8 \mathrm{~Hz}\right)$, $72.71\left(\mathrm{~d},{ }^{2} J_{\mathrm{C}-\mathrm{F}}=29.0 \mathrm{~Hz}\right), 67.97,64.67\left(\mathrm{~d},{ }^{2} J_{\mathrm{C}-\mathrm{F}}=24.7 \mathrm{~Hz}\right), 64.41 *\left(\mathrm{~d},{ }^{2} J_{\mathrm{C}-\mathrm{F}}=24.4 \mathrm{~Hz}\right), 53.31 *$, 52.87, 28.34*, 28.112. *Minor rotamer. $\mathrm{C}_{17} \mathrm{H}_{22} \mathrm{FNO}_{5}$, expected 339.2, found $\mathrm{m} / \mathrm{z}=240.1$, [M$\mathrm{Boc}+\mathrm{H}]^{+}$. 


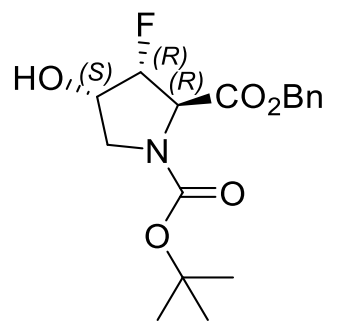

Compound $\mathbf{4 b}$ was obtained as a white solid in $25 \%$ isolated yield (30\% by $\left.{ }^{19} \mathrm{~F}-\mathrm{NMR}\right) .{ }^{1} \mathrm{H}-\mathrm{NMR}$ (500 $\left.\mathrm{MHz}, \mathrm{CDCl}_{3}\right) \delta:$ 7.38-7.35 (m, 5H), 5.29-5.11 (m, 2H), 4.99-4.88 (m, 1H), 4.64-4.47 (m, 1H), 4.41$4.36(\mathrm{~m}, 1 \mathrm{H}), 3.94-3.87(\mathrm{~m}, 1 \mathrm{H}), 3.37-3.27(\mathrm{~m}, 1 \mathrm{H}), 2.09$ (d, $\left.J_{\mathrm{H}-\mathrm{H}} 7.45 \mathrm{~Hz}\right), 1.47 *(9 \mathrm{H}), 1.33(9 \mathrm{H})$. ${ }^{19}$ F-NMR $\left(470.6 \mathrm{MHz}, \mathrm{CDCl}_{3}\right) \delta:-199.74,-200.29 * .{ }^{13} \mathrm{C}-\mathrm{NMR}\left(125.8 \mathrm{MHz}, \mathrm{CDCl}_{3}\right) \delta: 169.1\left(\mathrm{~d},{ }^{3} \mathrm{~J}\right.$ C-F $=13.1 \mathrm{~Hz}), 168.7^{*}\left(\mathrm{~d},{ }^{3} J_{\mathrm{C}-\mathrm{F}}=12.9 \mathrm{~Hz}\right), 154.07^{*}, 153.21,135.10^{*}, 134.9,128.8,128.6,128.5$, $128.2,94.0\left(\mathrm{~d},{ }^{1} J_{\mathrm{C}-\mathrm{F}}=190.0 \mathrm{~Hz}\right), 93.2 *\left(\mathrm{~d},{ }^{1} J_{\mathrm{C}-\mathrm{F}}=189.6 \mathrm{~Hz}\right), 80.9,70.3 *\left(\mathrm{~d},{ }^{2} J_{\mathrm{C}-\mathrm{F}}=18.2 \mathrm{~Hz}\right), 69.6$ $\left(\mathrm{d},{ }^{2} J_{\mathrm{C}-\mathrm{F}}=17.0 \mathrm{~Hz}\right), 67.6,63.7\left(\mathrm{~d},{ }^{2} J_{\mathrm{C}-\mathrm{F}}=23.9 \mathrm{~Hz}\right), 63.5^{*}\left(\mathrm{~d},{ }^{2} J_{\mathrm{C}-\mathrm{F}}=23.8 \mathrm{~Hz}\right), 49.8^{*}, 49.3,23.8^{*}$, 28.1. *Minor rotamer. $\mathrm{C}_{17} \mathrm{H}_{22} \mathrm{FNO}_{5}$, expected 339.2, found $\mathrm{m} / \mathrm{z}=240.1$, [M-Boc $\left.+\mathrm{H}\right]^{+}$.

\section{2-Benzyl 1-(tert-butyl) (2R,3S,4R)-3-fluoro-4-hydroxypyrrolidine-1,2-dicarboxylate (4a)}

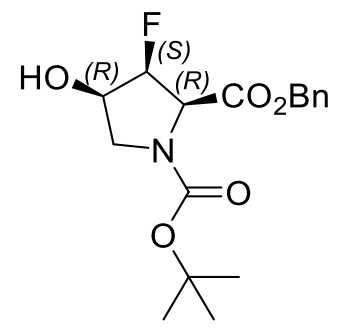

Compound $4 \mathrm{a}$ was obtained in $50 \%$ isolated yield as a white solid ( $58 \%$ by $\left.{ }^{19} \mathrm{~F}-\mathrm{NMR}\right) .{ }^{1} \mathrm{H}-\mathrm{NMR}$ $\left(500 \mathrm{MHz}, \mathrm{CDCl}_{3}\right) \delta: 7.37-7.32(\mathrm{~m}, 5 \mathrm{H}), 5.35-5.10(\mathrm{~m}, 3 \mathrm{H}), 4.64 *\left(\mathrm{dd},{ }^{2} J_{\mathrm{H}-\mathrm{F}}=21.0 \mathrm{~Hz}, J_{\text {Н-H }} 5.9 \mathrm{~Hz}\right.$, $1 \mathrm{H}), 4.54\left(\mathrm{dd},{ }^{2} J_{\mathrm{H}-\mathrm{F}}=21.7 \mathrm{~Hz}, J_{\mathrm{H}-\mathrm{H}} 5.9 \mathrm{~Hz}, 1 \mathrm{H}\right), 4.28(\mathrm{br} \mathrm{s}, 1 \mathrm{H}), 3.87\left(\mathrm{dd}, J_{\mathrm{H}-\mathrm{H}}=11.2 \mathrm{~Hz}, J_{\mathrm{H}-\mathrm{H}}=6.6\right.$ $\mathrm{Hz}, 1 \mathrm{H}), 3.82^{*}\left(\mathrm{dd}, J_{\mathrm{H}-\mathrm{H}}=11.1 \mathrm{~Hz}, J_{\mathrm{H}-\mathrm{H}}=6.6 \mathrm{~Hz}, 1 \mathrm{H}\right), 3.48-3.41(\mathrm{~m}, 1 \mathrm{H}), 2.75(\mathrm{br} \mathrm{s}, 1 \mathrm{H}), 1.46^{*}(\mathrm{~s}$, 9H), $1.32(\mathrm{~s}, 9 \mathrm{H}) .{ }^{19} \mathrm{~F}-\mathrm{NMR}\left(470.6 \mathrm{MHz}, \mathrm{CDCl}_{3}\right) \delta:-207.06,-207.67^{*} .{ }^{13} \mathrm{C}-\mathrm{NMR}(125.8 \mathrm{MHz}$, $\left.\mathrm{CDCl}_{3}\right) \delta: 168.3\left(\mathrm{~d},{ }^{3} J_{\mathrm{C}-\mathrm{F}}=7.0 \mathrm{~Hz}\right), 167.9^{*}\left(\mathrm{~d},{ }^{3} J_{\mathrm{C}-\mathrm{F}}=7.2 \mathrm{~Hz}\right), 153.9^{*}, 153.2,135.2^{*}, 135.0,128.6$, $128.5,128.4,128.3,128.2,91.5\left(\mathrm{~d},{ }^{1} J_{\mathrm{C}-\mathrm{F}}=189.6 \mathrm{~Hz}\right), 90.8^{*}\left(\mathrm{~d},{ }^{1} J_{\mathrm{C}-\mathrm{F}}=188.6 \mathrm{~Hz}\right), 81.1,70.6 *\left(\mathrm{~d},{ }^{2} J\right.$ $\left.\mathrm{C}-\mathrm{F}^{2} 17.7 \mathrm{~Hz}\right), 70.1\left(\mathrm{~d},{ }^{2} J_{\mathrm{C}-\mathrm{F}}=17.6 \mathrm{~Hz}\right), 67.6,61.6\left(\mathrm{~d},{ }^{2} J_{\mathrm{C}-\mathrm{F}}=21.9 \mathrm{~Hz}\right), 61.2^{*}\left(\mathrm{~d},{ }^{2} J_{\mathrm{C}-\mathrm{F}}=22.0 \mathrm{~Hz}\right)$, $50.6^{*}, 50.0,28.3 *$, 28.1. *Minor rotamer. $\mathrm{C}_{17} \mathrm{H}_{22} \mathrm{FNO}_{5}$, expected 339.2, found $\mathrm{m} / \mathrm{z}=240.1$, [M$\mathrm{Boc}+\mathrm{H}]^{+}$.

\section{Mitsunobu inversion, general procedure}


To a solution of fluoro-hydroxyproline $(0.435 \mathrm{mmol})$, triphenylphosphine $(342.0 \mathrm{mg}, 1.306 \mathrm{mmol})$ and 4-nitrobenzoic acid $(218.2 \mathrm{mg}, 1.306 \mathrm{mmol})$ in THF $(2 \mathrm{~mL})$, diisopropyl azodicarboxylate (DIAD) was added dropwise at $0{ }^{\circ} \mathrm{C}$. The flask was left at $0{ }^{\circ} \mathrm{C}$ for $4 \mathrm{~h}$, then the ice bath was removed and the mixture was stirred at r.t. for $24 \mathrm{~h}$. $\mathrm{Et}_{2} \mathrm{O}$ was added $(10 \mathrm{~mL})$ and the mixture was washed with saturated $\mathrm{NaHCO}_{3}(5 \mathrm{~mL})$. The mixture was washed with brine $(10 \mathrm{~mL})$, the organic phase was dried over anhydrous $\mathrm{MgSO}_{4}$. Volatiles were removed under reduced pressure to obtain yellow oil which was subjected to FCC (EtOAc/Heptane 1:9 to 3:7) to isolate the desired product. The product contained an impurity derived from the Mitsunobu reaction which could not be separated by further HPLC purification. However, separation from the impurity was possible after hydrolysis of the nitrobenzoate group.

\section{2-Benzyl 1-(tert-butyl) $(2 R, 3 S, 4 S)$-3-fluoro-4-((4-nitrobenzoyl)oxy) pyrrolidine-1,2-dicarboxylate}

(5)

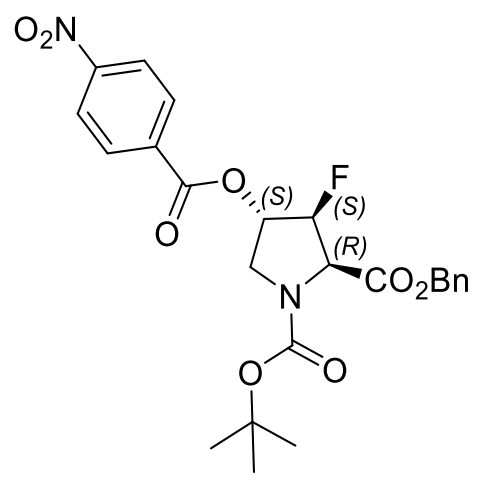

Compound $\mathbf{5}$ was obtained from compound $\mathbf{4 a}$ according to the general procedure for the Mitsunobu reaction as detailed hereinbefore $(187 \mathrm{mg}$, yield considered quantitative and was not calculated as product contained an inseparable impurity), as a pale yellow wax. ${ }^{1} \mathrm{H}-\mathrm{NMR}\left(500 \mathrm{MHz}, \mathrm{CDCl}_{3}\right) \delta$ : 8.31-8.29 (m, 2H), 8.17-8.15 (m, 2H), 7.38-7.35 (m, 5H), 5.57-5.54 (m, 1H), 5.45-5.33 (m, 1H), 5.31$5.16(\mathrm{~m}, 2 \mathrm{H}), 4.84^{*}\left(\mathrm{dd},{ }^{2} J_{\mathrm{H}-\mathrm{F}}=23.3 \mathrm{~Hz}, J_{\mathrm{H}-\mathrm{H}}=5.7 \mathrm{~Hz}, 1 \mathrm{H}\right), 4.72\left(\mathrm{dd},{ }^{2} J_{\mathrm{H}-\mathrm{F}}=24.3 \mathrm{~Hz}, J_{\mathrm{H}-\mathrm{H}}=5.5\right.$ $\mathrm{Hz}, 1 \mathrm{H}), 4.05-4.00(\mathrm{~m}, 1 \mathrm{H}), 3.90\left(\mathrm{~d}, J_{\mathrm{H}-\mathrm{H}}=12.8 \mathrm{~Hz}, 1 \mathrm{H}\right), 3.76^{*}\left(\mathrm{~d}, J_{\mathrm{H}-\mathrm{H}}=12.7 \mathrm{~Hz}\right), 1.46^{*}(\mathrm{~s}, 9 \mathrm{H})$, $1.36(\mathrm{~s}, 9 \mathrm{H}) .{ }^{19} \mathrm{~F}-\mathrm{NMR}\left(470.6 \mathrm{MHz}, \mathrm{CDCl}_{3}\right) \delta:-192.43,-193.28 * . *$ Minor rotamer. $\mathrm{C}_{24} \mathrm{H}_{25} \mathrm{FN}_{2} \mathrm{O}_{8}, \mathrm{rt}$ $=1.962 \mathrm{~min}$, expected 488.1, found $\mathrm{m} / \mathrm{z}=389.1,[\mathrm{M}-\mathrm{Boc}+\mathrm{H}]^{+}$. 

(S1)

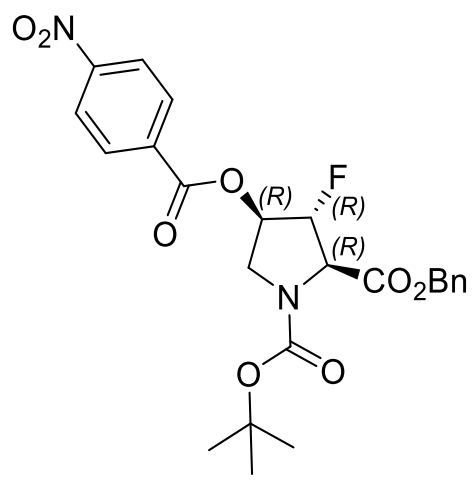

Prepared from compound $\mathbf{4 b}$ according to the general procedure for the Mitsunobu reaction as detailed hereinbefore $(30 \mathrm{mg}, 187 \mathrm{mg}$, yield considered quantitative and was not calculated as product contained an inseparable impurity, light yellow wax). ${ }^{1} \mathrm{H}$ NMR $\left(500 \mathrm{MHz}, \mathrm{CDCl}_{3}\right) 8.22-8.18$ (m, $2 \mathrm{H}), 8.02$ - $7.95(\mathrm{~m}, 2 \mathrm{H}), 7.26$ - $7.22(\mathrm{~m}, 5 \mathrm{H}), 5.57$ - $5.54(\mathrm{~m}, 1 \mathrm{H}), 5.34$ - $5.06(\mathrm{~m}, 3 \mathrm{H}), 4.89$ - 4.67 $(\mathrm{m}, 1 \mathrm{H}), 4.03-3.96(\mathrm{~m}, 1 \mathrm{H}), 3.88-3.74(\mathrm{~m}, 1 \mathrm{H}), 1.50 *$ (s, 9H), 1.40 (s. 9H). ${ }^{19} \mathrm{~F}-\mathrm{NMR}(470.6 \mathrm{MHz}$, $\mathrm{CDCl}_{3}$ ) $\delta:-182.16,-183.06 *$ * ${ }^{*}$ Minor rotamer. $\mathrm{C}_{24} \mathrm{H}_{25} \mathrm{FN}_{2} \mathrm{O}_{8}, \mathrm{HPLC}($ Method 1) rt = $1.975 \mathrm{~min}$, expected 488.1, found $\mathrm{m} / \mathrm{z}=389.1,[\mathrm{M}-\mathrm{Boc}+\mathrm{H}]^{+}$.

\section{2-Benzyl 1-(tert-butyl) (2R,3S,4S)-3-fluoro-4-hydroxypyrrolidine-1,2-dicarboxylate (4d)}

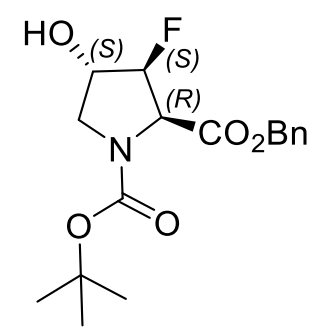

To a solution of the 4-nitrobenzonate ester $5(24.4 \mathrm{mg}, 0.05 \mathrm{mmol})$ in methanol $(1.0 \mathrm{~mL})$, sodium azide $(10 \mathrm{mg}, 0.15 \mathrm{mmol})$ was added under stirring. The mixture was heated and stirred to $50{ }^{\circ} \mathrm{C}$ until TLC analysis (EtOAc/Heptane 4:6) showed complete conversion of the starting material (15-30 min). The mixture was cooled to $0{ }^{\circ} \mathrm{C}$, brine was added $(1 \mathrm{~mL})$ and extracted with EtOAc $(3 \mathrm{X} 5 \mathrm{~mL})$. The organic phase was dried over anhydrous $\mathrm{MgSO}_{4}$, the volatile components (solvent) were removed under reduced pressure, to obtain a yellow oil which was subjected to FCC (EtOAc/Heptane 1:1) to isolate the desired product $\left(13.5 \mathrm{mg}, 80 \%\right.$ isolated yield) as a pale yellow wax. ${ }^{1} \mathrm{H}-\mathrm{NMR}(500 \mathrm{MHz}$, $\left.\mathrm{CDCl}_{3}\right) \delta: 7.36-7.35(\mathrm{~m}, 5 \mathrm{H}), 5.31-5.05(\mathrm{~m}, 3 \mathrm{H}), 4.74 *\left(\mathrm{dd},{ }^{2} J_{\text {H-F }}=24.3 \mathrm{~Hz}, J_{\text {H-H }} 5.1 \mathrm{~Hz}, 1 \mathrm{H}\right), 4.65$ $\left(\mathrm{dd},{ }^{2} J_{\mathrm{H}-\mathrm{F}}=25.5 \mathrm{~Hz}, J_{\mathrm{H}-\mathrm{H}} 5.2 \mathrm{~Hz}, 1 \mathrm{H}\right), 4.45-4.42(\mathrm{~m}, 1 \mathrm{H}), 3.77-3.74(\mathrm{~m}, 1 \mathrm{H}), 3.68-3.56(\mathrm{~m}, 1 \mathrm{H}), 2.33$ 
(br s, 1H), 1.46* (s, 9H), $1.33(\mathrm{~s}, 9 \mathrm{H}) .{ }^{19} \mathrm{~F}-\mathrm{NMR}\left(470.6 \mathrm{MHz}, \mathrm{CDCl}_{3}\right) \delta:-192.30,-192.41 * .{ }^{13} \mathrm{C}-$ NMR (125.8 MHz, $\left.\mathrm{CDCl}_{3}\right) \delta: 167.5\left(\mathrm{~d},{ }^{3} J_{\mathrm{C}-\mathrm{F}}=8.3 \mathrm{~Hz}\right), 167.3^{*}\left(\mathrm{~d},{ }^{3} J_{\mathrm{C}-\mathrm{F}}=10.8 \mathrm{~Hz}\right), 154.4 *, 153.9$, $135.4^{*}, 135.3,130.7,128.6,128.5,123.2,123.6,95.5\left(\mathrm{~d},{ }^{1} J_{\mathrm{C}-\mathrm{F}}=187.7 \mathrm{~Hz}\right), 94.8^{*}\left(\mathrm{~d},{ }^{1} J_{\mathrm{C}-\mathrm{F}}=\right.$ $187.3 \mathrm{~Hz}), 80.9,80.8^{*}, 72.8^{*}\left(\mathrm{~d},{ }^{2} J_{\mathrm{C}-\mathrm{F}}=28.0 \mathrm{~Hz}\right), 72.1\left(\mathrm{~d},{ }^{2} J_{\mathrm{C}-\mathrm{F}}=27.0 \mathrm{~Hz}\right), 67.2,62.4\left(\mathrm{~d},{ }^{2} J_{\mathrm{C}-\mathrm{F}}=21.2\right.$ $\mathrm{Hz}), 61.9^{*}\left(\mathrm{~d},{ }^{2} J_{\mathrm{C}-\mathrm{F}}=21.0 \mathrm{~Hz}\right), 52.1 *, 51.8,28.4 *, 28.1$. $*$ Minor rotamer. $\mathrm{C}_{17} \mathrm{H}_{22} \mathrm{FNO}_{5}$, expected 339.2, found $\mathrm{m} / \mathrm{z}=240.1,[\mathrm{M}-\mathrm{Boc}+\mathrm{H}]^{+}$.

\section{General procedure for the de-benzylation of benzylester}

The selected benzyl ester $\mathbf{4 a}, \mathbf{4 b}, \mathbf{4} \mathbf{c}$ or $\mathbf{4 d}(85 \mathrm{mg}, 0.250 \mathrm{mmol})$ was dissolved in a mixture of $\mathrm{MeOH} / \mathrm{THF} 1: 2(15 \mathrm{~mL})$, a catalytic amount of palladium over carbon $(10 \%$, dry) was added and the mixture was stirred under hydrogen atmosphere until TLC analysis (EtOAc/Heptane 1:1) showed complete conversion of the starting material. The mixture was then filtered on a celite pad and the solvents were removed in vacuum to obtain the de-benzylated product which was used directly in the next stage without further purification. Analytically pure samples were prepared by preparative HPLC purification.

(2R,3S,4R)-1-(tert-butoxycarbonyl)-3-fluoro-4-hydroxypyrrolidine-2-carboxylic acid (6a)

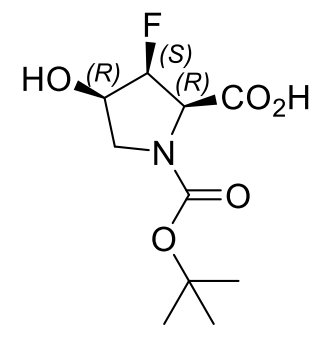

Starting from the corresponding benzyl ester $4 \mathbf{a}$ using the de-benzylation procedure detailed hereinbefore, compound $\mathbf{6 d}$ was obtained as a white solid $(85 \mathrm{mg})$ in $99 \%$ isolated yield. ${ }^{1} \mathrm{H}-\mathrm{NMR}$ $\left(500 \mathrm{MHz}, \mathrm{CD}_{3} \mathrm{OD}\right) \delta: 5.21-5.05(\mathrm{~m}, 1 \mathrm{H}), 4.54\left(\mathrm{dd}, J_{\mathrm{H}-\mathrm{H}}=4.7 \mathrm{~Hz},{ }^{2} J_{\mathrm{H}-\mathrm{F}}=28.9 \mathrm{~Hz}, 1 \mathrm{H}\right), 4.31-4.19$ $(\mathrm{m}, 1 \mathrm{H}), 3.87-3.82(\mathrm{~m}, 1 \mathrm{H}), 3.32-3.26(\mathrm{~m}, 1 \mathrm{H}), 1.48^{*}(\mathrm{~s}, 9 \mathrm{H}), 1.43(\mathrm{~s}, 9 \mathrm{H}) .{ }^{19} \mathrm{~F}-\mathrm{NMR}(470.6 \mathrm{MHz}$ $\left.\mathrm{CD}_{3} \mathrm{OD}\right) \delta:-208.58,-208.69^{*} .{ }^{13} \mathrm{C}-\mathrm{NMR}\left(125.8 \mathrm{MHz} \mathrm{CD}_{3} \mathrm{OD}\right) \delta: 169.3\left(\mathrm{~d},{ }^{3} J_{\mathrm{C}-\mathrm{F}}=8.0 \mathrm{~Hz}\right), 168.8^{*}$ $\left(\mathrm{d},{ }^{3} J_{\mathrm{C}-\mathrm{F}}=8.5 \mathrm{~Hz}\right), 154.6^{*}, 154.2,92.2\left(\mathrm{~d},{ }^{1} J_{\mathrm{C}-\mathrm{F}}=186.9 \mathrm{~Hz},\right) 91.5^{*}\left(\mathrm{~d},{ }^{1} J_{\mathrm{C}-\mathrm{F}}=186.2 \mathrm{~Hz}\right), 80.6,80.5^{*}$, $69.6^{*}\left(\mathrm{~d},{ }^{2} J_{\mathrm{C}-\mathrm{F}}=18.0 \mathrm{~Hz}\right), 69.3\left(\mathrm{~d},{ }^{2} J_{\mathrm{C}-\mathrm{F}}=17.9 \mathrm{~Hz}\right), 62.6\left(\mathrm{~d},{ }^{2} J_{\mathrm{C}-\mathrm{F}}=20.8 \mathrm{~Hz}\right), 62.0(\mathrm{~d}, 2 \mathrm{~J} \mathrm{C}-\mathrm{F}=20.1$ $\mathrm{Hz}), 27.2^{*}, 27.0$. $*$ Minor rotamer. $\mathrm{C}_{10} \mathrm{H}_{16} \mathrm{FNO}_{5}$, expected 249.1, found $\mathrm{m} / \mathrm{z}=150.1$, [M-Boc $\left.+\mathrm{H}\right]^{+}$. $[\alpha]_{\mathrm{D}}^{25}=-30.5\left(c=1, \mathrm{CHCl}_{3}\right)$. 


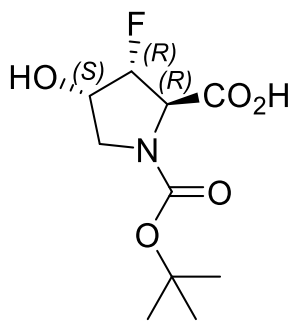

Starting from the corresponding benzyl ester $\mathbf{4 b}$, using the de-benzylation procedure detailed hereinbefore, compound $\mathbf{6 b}$ was obtained as a white solid $(62 \mathrm{mg})$ in $99 \%$ isolated yield. ${ }^{1} \mathrm{H}-\mathrm{NMR}$ (500 MHz, $\left.\mathrm{CD}_{3} \mathrm{OD}\right) \delta:$ 5.01-4.93 (m, 1H), 4.43-4.27 (m, 2H), 3.78-3.74 (m, 1H), 3.30-3.28 (m, $\left.1 \mathrm{H}\right)$, $1.49 *(\mathrm{~s}, 9 \mathrm{H}), 1.44(\mathrm{~s}, 9 \mathrm{H}) .{ }^{19} \mathrm{~F}-\mathrm{NMR}\left(470.6 \mathrm{MHz}, \mathrm{CD}_{3} \mathrm{OD}\right) \delta:-199.02,-199.10 * .{ }^{13} \mathrm{C}-\mathrm{NMR}(125.8$ $\mathrm{MHz} \mathrm{CD} \mathrm{CD}_{3} \mathrm{OD} \delta: 171.2\left(\mathrm{~d},{ }^{3} J_{\mathrm{C}-\mathrm{F}}=11.9 \mathrm{~Hz}\right), 170.6^{*}\left(\mathrm{~d},{ }^{3} J_{\mathrm{C}-\mathrm{F}}=12.9 \mathrm{~Hz}\right), 163.0,154.7^{*}, 154.2,94.0$ $\left(\mathrm{d},{ }^{1} J_{\mathrm{C}-\mathrm{F}}=191.8 \mathrm{~Hz}\right), 93.3^{*}\left(\mathrm{~d},{ }^{1} J_{\mathrm{C}-\mathrm{F}}=191.1 \mathrm{~Hz}\right), 80.8,80.6^{*}, 69.3^{*}\left(\mathrm{~d},{ }^{2} J_{\mathrm{C}-\mathrm{F}}=17.4 \mathrm{~Hz}\right), 68.8\left(\mathrm{~d},{ }^{2} J_{\mathrm{C}-}\right.$ $\mathrm{F}=17.3 \mathrm{~Hz}), 64.0\left(\mathrm{~d},{ }^{2} J_{\mathrm{C}-\mathrm{F}}=23.6 \mathrm{~Hz}\right), 63.7^{*}\left(\mathrm{~d},{ }^{2} J_{\mathrm{C}-\mathrm{F}}=23.3 \mathrm{~Hz}\right), 27.2^{*}, 27.1 . *$ Minor rotamer. $\mathrm{C}_{10} \mathrm{H}_{16} \mathrm{FNO}_{5}$, expected 249.1, found $\mathrm{m} / \mathrm{z}=150.1$, $[\mathrm{M}-\mathrm{Boc}+\mathrm{H}]^{+} .[\alpha]_{\mathrm{D}}^{25}=-19.3\left(c=1, \mathrm{CHCl}_{3}\right)$.

$(2 R, 3 R, 4 R)-1$-(tert-butoxycarbonyl)-3-fluoro-4-hydroxypyrrolidine-2-carboxylic acid (6c)

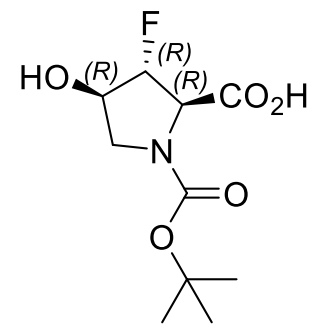

Starting from the corresponding benzyl ester $4 \mathbf{c}$ using the de-benzylation procedure detailed hereinbefore, compound $\mathbf{6 c}$ was obtained as a white solid $(43 \mathrm{mg})$ in $99 \%$ isolated yield. ${ }^{1} \mathrm{H}-\mathrm{NMR}$ (500 MHz, CD 3 OD) $\delta:{ }^{1} \mathrm{H}$ NMR (500 MHz, MeOD) d $5.05(\mathrm{dd}, \mathrm{J}=6.3,49.9 \mathrm{~Hz}, 1 \mathrm{H}), 4.50$ - 4.40 (m, 1H), 4.34 - $4.29(\mathrm{~m}, 1 \mathrm{H}), 3.73-3.68(\mathrm{~m}, 1 \mathrm{H}), 3.46$ (d, J=11.6 Hz, 1H), 1.52* (s, 9H), 1.46 (s, 9H). ${ }^{19}$ F-NMR (470.6 MHz, $\left.\mathrm{CD}_{3} \mathrm{OD}\right) \delta:-183.44,-183.58 * .{ }^{13} \mathrm{C}-\mathrm{NMR}\left(125.8 \mathrm{MHz} \mathrm{CD}_{3} \mathrm{OD}\right) \delta: 170.2\left(\mathrm{~d},{ }^{3} J\right.$ C-F $=14.0 \mathrm{~Hz}), 169.8^{*}\left(\mathrm{~d},{ }^{3} J_{\mathrm{C}-\mathrm{F}}=14.6 \mathrm{~Hz}\right), 163.0,155.0^{*}, 154.7,98.2 *\left(\mathrm{~d},{ }^{1} J_{\mathrm{C}-\mathrm{F}}=186.2 \mathrm{~Hz}\right), 97.4^{*}$ $\left(\mathrm{d},{ }^{1} J_{\mathrm{C}-\mathrm{F}}=186.3 \mathrm{~Hz}\right), 80.5,80.4^{*}, 72.2^{*}\left(\mathrm{~d},{ }^{2} J_{\mathrm{C}-\mathrm{F}}=27.3 \mathrm{~Hz}\right), 71.4\left(\mathrm{~d},{ }^{2} J_{\mathrm{C}-\mathrm{F}}=27.1 \mathrm{~Hz}\right), 64.3\left(\mathrm{~d},{ }^{2} J_{\mathrm{C}-\mathrm{F}}\right.$ $=23.6 \mathrm{~Hz}), 63.9^{*}\left(\mathrm{~d},{ }^{2} J_{\mathrm{C}-\mathrm{F}}=23.4 \mathrm{~Hz}\right), 52.2^{*}, 51.6,27.3^{*}, 27.1 . \mathrm{C}_{10} \mathrm{H}_{16} \mathrm{FNO}_{5}$, expected 249.1, found $\mathrm{m} / \mathrm{z}=150.1,[\mathrm{M}-\mathrm{Boc}+\mathrm{H}]^{+} .[\alpha]_{\mathrm{D}}^{25}=-9.5\left(c=1, \mathrm{CHCl}_{3}\right)$. 
<smiles>O=C(O)[C@@H]1[C@H](F)[C@@H](O)CN1C(=O)O</smiles>

Starting from the corresponding benzyl ester 4d using the de-benzylation procedure detailed hereinbefore, compound $\mathbf{6 d}$ was obtained as a white solid $(62 \mathrm{mg})$ in $99 \%$ isolated yield. ${ }^{1} \mathrm{H}-\mathrm{NMR}$ (500 MHz, $\left.\mathrm{CD}_{3} \mathrm{OD}\right) \delta:$ 5.13-4.99 (m, 1H), 4.61-4.50 (m, 1H), 4.30-7.27 (m, 1H), 3.61-3.54 (m, 2H), $1.476 *(\mathrm{~s}, 9 \mathrm{H}), 1.44$ (s, 9H). ${ }^{19} \mathrm{~F}-\mathrm{NMR}\left(470.6 \mathrm{MHz} \mathrm{CD}_{3} \mathrm{OD}\right) \delta:-191.59,-191.73 * .{ }^{13} \mathrm{C}-\mathrm{NMR}(125.8$ $\left.\mathrm{MHz} \mathrm{CD}_{3} \mathrm{OD}\right) \delta: 169.6\left(\mathrm{~d},{ }^{3} J_{\mathrm{C}-\mathrm{F}}=8.5 \mathrm{~Hz}\right), 169.2 *\left(\mathrm{~d},{ }^{3} J_{\mathrm{C}-\mathrm{F}}=8.4 \mathrm{~Hz}\right), 155.0 *, 154.6,95.5\left(\mathrm{~d},{ }^{1} J_{\mathrm{C}-\mathrm{F}}=\right.$ $186.0 \mathrm{~Hz}), 95.2 *\left(\mathrm{~d},{ }^{1} J_{\mathrm{C}-\mathrm{F}}=128.5 \mathrm{~Hz}\right), 80.6,80.4^{*}, 71.9^{*}\left(\mathrm{~d},{ }^{2} J_{\mathrm{C}-\mathrm{F}}=26.6 \mathrm{~Hz}\right), 71.3\left(\mathrm{~d},{ }^{2} J_{\mathrm{C}-\mathrm{F}}=26.7\right.$ $\mathrm{Hz}), 62.4\left(\mathrm{~d},{ }^{2} J_{\mathrm{C}-\mathrm{F}}=21.3 \mathrm{~Hz}\right), 62.0 *\left(\mathrm{~d},{ }^{2} J_{\mathrm{C}-\mathrm{F}}=21.6 \mathrm{~Hz}\right), 52.2 *, 51.3,27.3 *$, 27.0. *Minor rotamer. $\mathrm{C}_{10} \mathrm{H}_{16} \mathrm{FNO}_{5}$, expected 249.1, found $\mathrm{m} / \mathrm{z}=150.1,[\mathrm{M}-\mathrm{Boc}+\mathrm{H}]^{+} .[\alpha]_{\mathrm{D}}^{25}=-39.2\left(c=1, \mathrm{CHCl}_{3}\right)$.

\section{(2R,3R,4S)-1-(((9H-fluoren-9-yl)methoxy)carbonyl)-3-fluoro-4-hydroxypyrrolidine-2-carboxylic} acid (S2)

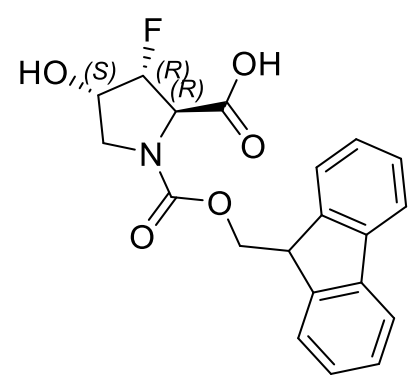

Compound $6 \mathbf{b}(67 \mathrm{mg}, 0.36 \mathrm{mmol})$ was Boc deprotected using a solution of $4 \mathrm{~N} \mathrm{HCl}$ in Dioxane (2 $\mathrm{mL})$ in DCM (2 mL). Solvents were evaporated and the crude product was dissolved in a mixture of water/dioxane $(1: 1,4 \mathrm{~mL})$. Sodium hydrogenocarbonate $(94 \mathrm{mg}, 1.1 .6 \mathrm{mmol})$ was added and the mixture was stirred at room temperature for $10 \mathrm{~min}$. 9-Fluorenylmethyl $\mathrm{N}$-succinimidyl carbonate (121 $\mathrm{mg}, 0.36 \mathrm{mmol})$ was added in small portions and the mixture was stirred at room temperature overnight. The reaction was cooled to $0{ }^{\circ} \mathrm{C}$ and treated with $\mathrm{KHSO}_{4}(5 \%)$ to $\mathrm{pH}=3-4$. The desired product was extracted with ethyl acetate $(3 \mathrm{X} 15 \mathrm{~mL})$ and the organic phase was dried over $\mathrm{MgSO} 4$. Solvents were evaporated to obtain the crude product which can be further purified by column chromatography on silica using a gradient from $5 \%$ to $20 \%$ of $\mathrm{MeOH}$ in DCM. (Obtained $100 \mathrm{mg}$, $75 \%$ yield). MS analysis: $\mathrm{C}_{20} \mathrm{H}_{18} \mathrm{FNO}$, expected 371.4 , found $372.4\left[\mathrm{M}+\mathrm{H}^{+}\right]$ 
${ }^{1} \mathrm{H}$ NMR (500 MHz, $\mathrm{CDCl}_{3}$ ) (mixture of rotamers) $\delta: 7.76-7.69(\mathrm{~m}, 2 \mathrm{H}), 7.56-7.49(\mathrm{~m}, 2 \mathrm{H}), 7.41$ 7.27 (m, 4H), $5.21-4.93(\mathrm{~m}, 2 \mathrm{H}), 4.61(\mathrm{~d}, \mathrm{~J}=18.6 \mathrm{~Hz}, 1 \mathrm{H}), 4.46-4.37(\mathrm{~m}, 3 \mathrm{H}), 4.25-4.10(\mathrm{~m}, 1 \mathrm{H})$, $3.91-3.84(\mathrm{~m}, 1 \mathrm{H}), 3.40\left(\mathrm{t}, J_{\mathrm{H}-\mathrm{H}}=9.3 \mathrm{~Hz}, 1 \mathrm{H}\right) .{ }^{19} \mathrm{~F} \mathrm{NMR}\left(470.6 \mathrm{MHz}, \mathrm{CDCl}_{3}\right) \delta:-199.35,-201.73$. 13C NMR (125.8 MHz, $\left.\mathrm{CDCl}_{3}\right) \delta: 172.0,171.9,170.9,170.8,156.4,154.6,143.7,143.6,141.6$, $141.6,128.2,127.4,125.2,125.2,120.3,93.8,92.3,70.2,70.1,69.7,69.5,68.8,68.2,64.0,63.8,63.6$, $63.4,49.6,47.2$.

(2R,3S,4S)-1-(((9H-fluoren-9-yl)methoxy)carbonyl)-3-fluoro-4-hydroxypyrrolidine-2-carboxylic acid (S3)

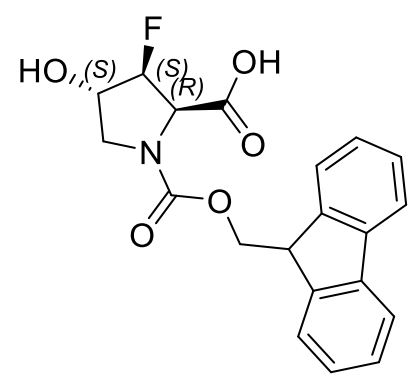

Compound $\mathbf{S 3}$ was prepared accordingly to the procedure described for the preparation of compound S2, starting from 6d (Obtained $106 \mathrm{mg}, 79 \%$ yield). MS analysis: $\mathrm{C}_{20} \mathrm{H}_{18} \mathrm{FNO}$, expected 371.4, found $372.4\left[\mathrm{M}+\mathrm{H}^{+}\right]$

${ }^{1} \mathrm{H}$ NMR $\left(500 \mathrm{MHz}, \mathrm{CDCl}_{3} / \mathrm{CD}_{3} \mathrm{OD} 8: 2\right)$ mixture of rotamers, $\delta: 7.73-7.69(\mathrm{~m}, 2 \mathrm{H}), 7.59-7.52(\mathrm{~m}$, 2H), 7.37 - $7.31(\mathrm{~m}, 2 \mathrm{H}), 7.29$ - $7.24(\mathrm{~m}, 2 \mathrm{H}), 5.18$ - $5.02(\mathrm{~m}, 1 \mathrm{H}), 4.75$ - $4.61(\mathrm{~m}, 1 \mathrm{H}), 4.38$ - $4.14(\mathrm{~m}$, $4 \mathrm{H}), 3.79$ - 3.57 (m, 4H). ${ }^{19} \mathrm{~F}-\mathrm{NMR}\left(470.6 \mathrm{MHz}, \mathrm{CDCl}_{3} / \mathrm{CD}_{3} \mathrm{OD} 8: 2\right) \delta:-188.40,-188.58 .{ }^{13} \mathrm{C} \mathrm{NMR}$ $\left(125.8 \mathrm{MHz}, \mathrm{CDCl}_{3} / \mathrm{CD}_{3} \mathrm{OD}\right.$ 8:2) $\delta: 172.6,155.3,155.2,143.7,143.6,143.5,143.4,141.0,141.0$, $140.9,127.5,127.5,126.9,124.9,124.9,119.7,119.7,96.2,95.3,94.7,93.8,71.9,71.7,71.2,71.0$, $68.0,67.7,66.8,62.3,62.1,62.1,57.5,52.4,52.1$.

\section{Methyl (2R,3S)-3-fluoro-4-0xo-1-(9-phenyl-9H-fluoren-9-yl)pyrrolidine-2-carboxylate (8)}<smiles>CC(=O)[C@H]1[C@@H](F)C(=O)CN1C1(c2ccccc2)c2ccccc2-c2ccccc21</smiles>

A solution of starting ketone $6(600 \mathrm{mg}, 1.57 \mathrm{mmol})$ in DCM $(15 \mathrm{~mL})$ was cooled to $-30{ }^{\circ} \mathrm{C}$, and TEA $(700 \mu \mathrm{L}, 4.70 \mathrm{mmol})$ was added dropwise. Trimethylsilyltriflate (TMSOTf) $(570 \mu \mathrm{L}, 3.13 \mathrm{mmol})$ 
was then added dropwise at $-30{ }^{\circ} \mathrm{C}$. The mixture was stirred for $2 \mathrm{~h}$ and kept below $-10{ }^{\circ} \mathrm{C}$. The solvent was then removed under vacuum and pentane $(25 \mathrm{~mL})$ was added. The mixture was poured into a saturated solution of $\mathrm{NaHCO}_{3}(50 \mathrm{~mL})$ and was vigorously shaken. The organic layer was separated, washed with brine then dried over anhydrous $\mathrm{MgSO}_{4}$. The solvent (volatiles) was removed under reduced pressure to obtain a light yellow oil, corresponding to a non-isolated intermediate trimethylsilylenolate, which was dissolved in acetonitrile $(30 \mathrm{~mL})$ and cooled to $-40{ }^{\circ} \mathrm{C}$. Selectfluor (834 mg, $2.35 \mathrm{mmol}$ ) was added in one portion and the mixture was allowed to reach $10{ }^{\circ} \mathrm{C}$ overnight. TLC analysis (EtOAc/Heptane 2:8) showed complete conversion of the starting material. A saturated solution of $\mathrm{NH}_{4} \mathrm{Cl}$ was added (30 ML), the resulting mixture was vigorously shaken and the organic layer was then separated. The aqueous layer was extracted with EtOAc $(2 \mathrm{X} 30 \mathrm{~mL})$ and the organic phase was washed with brine, then dried over $\mathrm{MgSO}_{4}$. Volatiles were removed under reduced pressure to obtain the crude product as yellow oil, which was purified by FCC (EtOAc/Heptane 1:9) to obtain the desired fluoroketone (346 mg, 55\% yield) as a white solid. MS analysis does not show any informative result as the only ion detected corresponded to the 9-phenylfluorenyl carbocation $\mathrm{m} / \mathrm{z}$ $=241.1$.

${ }^{1} \mathrm{H}-\mathrm{NMR}\left(500 \mathrm{MHz}, \mathrm{CDCl}_{3}\right) \delta: 7.76\left(\mathrm{~d}, J_{\mathrm{H}-\mathrm{H}}=7.6 \mathrm{~Hz}, 1 \mathrm{H}\right), 7.71\left(\mathrm{~d}, J_{\mathrm{H}-\mathrm{H}}=7.6 \mathrm{~Hz}, 1 \mathrm{H}\right), 7.46-7.325$ $(\mathrm{m}, 11 \mathrm{H}), 5.07\left(\mathrm{dd},{ }^{1} J_{\mathrm{H}-\mathrm{F}}=51.0 \mathrm{~Hz}, J_{\mathrm{H}-\mathrm{H}}=7.9 \mathrm{~Hz}, 1 \mathrm{H}\right), 4.03\left(\mathrm{~d}, J_{\mathrm{H}-\mathrm{H}}=8.1 \mathrm{~Hz}, 1 \mathrm{H}\right), 3.98\left(\mathrm{~d}, J_{\mathrm{H}-\mathrm{H}}=\right.$ $17.9 \mathrm{~Hz}, 1 \mathrm{H}), 3.63\left(\mathrm{~d}, J_{\mathrm{H}-\mathrm{H}}=17.9 \mathrm{~Hz}, 1 \mathrm{H}\right), 3.19(\mathrm{~s}, 3 \mathrm{H}) .{ }^{19} \mathrm{~F}-\mathrm{NMR}\left(470.6 \mathrm{MHz}, \mathrm{CDCl}_{3}\right) \delta:-206.52$. ${ }^{13} \mathrm{C}-\mathrm{NMR}\left(125.8 \mathrm{MHz}, \mathrm{CDCl}_{3}\right) \delta: 205.4\left(\mathrm{~d},{ }^{3} J_{\mathrm{C}-\mathrm{F}}=12.7 \mathrm{~Hz}\right), 169.3,124.2,144.7,141.2,140.6,139.9$, $129.3,129.2,128.8,123.3,128.0,127.7,126.8,126.6,125.1,120.5,120.4,89.1\left(\mathrm{~d},{ }^{1} J_{\mathrm{C}-\mathrm{F}}=205.4 \mathrm{~Hz}\right)$, $75.1,60.0\left(\mathrm{~d},{ }^{2} J_{\mathrm{C}-\mathrm{F}}=20.3 \mathrm{~Hz}\right), 51.8\left(\mathrm{~d},{ }^{2} J_{\mathrm{C}-\mathrm{F}}=22.8 \mathrm{~Hz}\right)$.

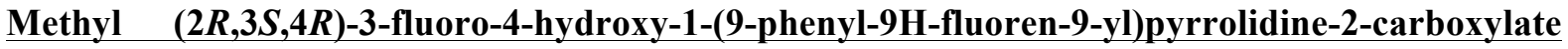
(10)

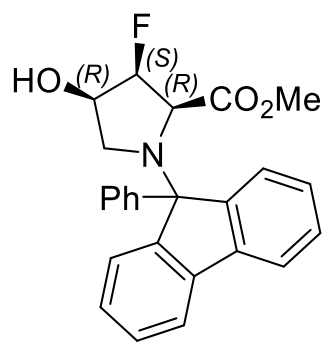

Fluoroketone $10(638 \mathrm{mg}, 1.59 \mathrm{mmol})$ was dissolved in THF/EtOH 1:1 $(20 \mathrm{~mL})$ at $0{ }^{\circ} \mathrm{C} . \mathrm{NaBH}_{4}(63$ $\mathrm{mg}, 1.67 \mathrm{mmol}$ ) was added portion-wise and the reaction mixture was stirred for $1 \mathrm{~h}$ at $0{ }^{\circ} \mathrm{C}$. TLC analysis (EtOAc/Heptane 3:7) showed complete conversion of the starting material. The reaction mixture was concentrated under vacuum, EtOAc was added $(30 \mathrm{~mL})$ and a solution of $\mathrm{NaHSO}_{4}(5 \%)$ 
was added dropwise until a $\mathrm{pH}$ of 3 to 4 was obtained. The acidified mixture was washed with brine $(10 \mathrm{~mL})$, and the organic phase was dried over anhydrous $\mathrm{MgSO}_{4}$. Volatiles were removed under reduced pressure to obtain the title compound as a white solid in quantitative yield.

${ }^{1} \mathrm{H}-\mathrm{NMR}\left(500 \mathrm{MHz}, \mathrm{CDCl}_{3}\right) \delta: 7.79\left(\mathrm{~d}, J_{\mathrm{H}-\mathrm{H}}=7.6 \mathrm{~Hz}, 1 \mathrm{H}\right), 7.65\left(\mathrm{~d}, J_{\mathrm{H}-\mathrm{H}}=7.5 \mathrm{~Hz}, 1 \mathrm{H}\right), 7.55-7.53$ (m, 2H), 7.49-7.48 (m 1H), 7.44-7.42 (m, 1H), 7.36-7.33 (m, 1H), 7.30-7.26 (m, 5H), 7.15-7.12 (m, $1 \mathrm{H}), 4.76-4.64(\mathrm{~m}, 1 \mathrm{H}), 4.15(\mathrm{br} \mathrm{s}, 2 \mathrm{H}), 3.45\left(\mathrm{dd},{ }^{2} J_{\mathrm{H}-\mathrm{F}}=10.6 \mathrm{~Hz}, J_{\mathrm{H}-\mathrm{H}}=5.0 \mathrm{~Hz}, 1 \mathrm{H}\right), 3.39(\mathrm{~s}, 3 \mathrm{H})$, $3.27\left(\mathrm{dd}, J_{\mathrm{H}-\mathrm{H}}=8.8 \mathrm{~Hz}, J_{\mathrm{H}-\mathrm{H}}=6.3 \mathrm{~Hz}, 1 \mathrm{H}\right), 3.05\left(\mathrm{~d}, J_{\mathrm{H}-\mathrm{H}}=10.6 \mathrm{~Hz}, 1 \mathrm{H}\right) .{ }^{19} \mathrm{~F}-\mathrm{NMR}(470.6 \mathrm{MHz}$, $\left.\mathrm{CDCl}_{3}\right) \delta$ : -205.26. ${ }^{13} \mathrm{C}-\mathrm{NMR}\left(125.8, \mathrm{CDCl}_{3}\right) \delta: 173.8\left(\mathrm{~d},{ }^{3} J_{\mathrm{C}-\mathrm{F}}=4.6 \mathrm{~Hz}\right), 147.4,144.6,141.9,140.8$, $139.2,129.1,128.7,128.6,127.9,127.7,127.4,126.0,126.3,120.4,120.1,90.7$ (d, $\left.{ }^{1} J_{\mathrm{C}-\mathrm{F}}=197.6 \mathrm{~Hz}\right)$, 75.6, $70.8\left(\mathrm{~d},{ }^{2} J_{\mathrm{C}-\mathrm{F}}=16.1 \mathrm{~Hz}\right), 60.8\left(\mathrm{~d},{ }^{2} J_{\mathrm{C}-\mathrm{F}}=21.8 \mathrm{~Hz}\right), 52.9\left(\mathrm{~d},{ }^{3} J_{\mathrm{C}-\mathrm{F}}=3.5 \mathrm{~Hz}\right), 52.2,29.7$.

\section{Methyl $(2 R, 3 S, 4 S)$-3-fluoro-4-((4-nitrobenzoyl)oxy)-1-(9-phenyl-9H-fluoren-9-yl)pyrrolidine-2-} carboxylate (11)

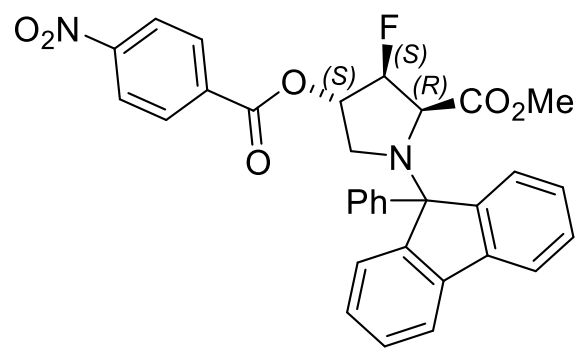

Compound $\mathbf{1 0}$ was prepared from preparative compound $\mathbf{5}$ according to the general procedure for the Mitsunobu inversion as detailed before. Compound 11 was obtained in $87 \%$ yield $(92 \mathrm{mg})$.

${ }^{1} \mathrm{H}-\mathrm{NMR}\left(500 \mathrm{MHz}, \mathrm{CDCl}_{3}\right)$ 8: 8.30-8.28 (m, 2H), 8.14-8.12 (m, 2H), 7.74-7.73 (m, 1H), 7.69-7.68 (m, 1H), 7.52-7.50 (m, 2H), 7.40-7.38 (m, 4H), 7.28-7.18 (m, 5H), 5.75-5.68 (m, 1H), $5.06\left(\mathrm{ddd},{ }^{1} J_{\mathrm{H}-}\right.$ $\left.\mathrm{F}=53.5 \mathrm{~Hz}, J_{\mathrm{H}-\mathrm{H}}=8.0 \mathrm{~Hz}, J_{\mathrm{H}-\mathrm{H}}=5.5 \mathrm{~Hz}, 1 \mathrm{H}\right), 4.04\left(\mathrm{dd},{ }^{2} J_{\mathrm{H}-\mathrm{F}}=10.2 \mathrm{~Hz}, J_{\mathrm{H}-\mathrm{H}}=6.8 \mathrm{~Hz}, 1 \mathrm{H}\right), 3.67$ $\left(\mathrm{dd}, J_{\mathrm{H}-\mathrm{H}}=7.9 \mathrm{~Hz}, J_{\mathrm{H}-\mathrm{H}}=6.7 \mathrm{~Hz}, 1 \mathrm{H}\right), 3.36(\mathrm{~s}, 3 \mathrm{H}), 3.08\left(\mathrm{dd}, J_{\mathrm{H}-\mathrm{H}}=10.5 \mathrm{~Hz}, J_{\mathrm{H}-\mathrm{H}}=5.6 \mathrm{~Hz}, 1 \mathrm{H}\right)$. ${ }^{19} \mathrm{~F}-\mathrm{NMR}\left(470.6 \mathrm{MHz} \mathrm{CDCl}_{3}\right) \delta:-196.44 .{ }^{13} \mathrm{C}-\mathrm{NMR}\left(125.8, \mathrm{CDCl}_{3}\right) \delta: 170.3\left(\mathrm{~d},{ }^{3} J_{\mathrm{C}-\mathrm{F}}=4.5 \mathrm{~Hz}\right)$, 163.9, 150.8, 146.1, 145.6, 141.1, 140.1, 134.7, 130.9, 129.0, 128.6, 127.9, 127.8, 127.4, 127.1, 126.0, 123.6, 120.3, 120.1, $93.3\left(\mathrm{~d},{ }^{1} J_{\mathrm{C}-\mathrm{F}}=195.3 \mathrm{~Hz}\right), 75.4,61.9\left(\mathrm{~d},{ }^{2} J_{\mathrm{C}-\mathrm{F}}=21.7 \mathrm{~Hz}\right), 51.7,49.7\left(\mathrm{~d},{ }^{3} J_{\mathrm{C}-\mathrm{F}}=\right.$ $5.6 \mathrm{~Hz})$. 
(S4)<smiles>CC(=O)[C@H]1[C@@H](O)[C@@H](O)CN1C1(c2ccccc2)c2ccccc2-c2ccccc21</smiles>

To a stirred solution of the 4-nitrobenzoate ester $11(217 \mathrm{mg}, 0.391 \mathrm{mmol})$ in THF $(8 \mathrm{~mL})$ at $0{ }^{\circ} \mathrm{C}$, $\mathrm{LiOH}(19 \mathrm{mg}, 0.469 \mathrm{mmol})$ dissolved in water $(2 \mathrm{~mL})$ was added dropwise. The mixture was stirred at $0{ }^{\circ} \mathrm{C}$ for $2 \mathrm{~h}$. TLC analysis (EtOAc/Heptane 3:7) showed complete conversion of the starting material. The reaction mixture was concentrated under vacuum, water $(5 \mathrm{~mL})$ was added and the mixture was extracted with EtOAc ( 3 X $15 \mathrm{~mL})$. The organic phase was washed with brine $(5 \mathrm{~mL})$, dried over anhydrous $\mathrm{MgSO}_{4}$ and the volatiles were removed under reduced pressure. The crude product was purified by FCC (EtOAc/Heptane 1:9) to obtain the title compound as white foam in quantitative yield.

${ }^{1} \mathrm{H}-\mathrm{NMR}\left(500 \mathrm{MHz}, \mathrm{CDCl}_{3}\right) \delta: 7.75\left(\mathrm{~d}, J_{\mathrm{H}-\mathrm{H}}=7.5 \mathrm{~Hz}, 1 \mathrm{H}\right), 7.65\left(\mathrm{~d}, J_{\mathrm{H}-\mathrm{H}}=7.5 \mathrm{~Hz}, 1 \mathrm{H}\right), 7.54-7.52$ $(\mathrm{m}, 2 \mathrm{H}), 7.49\left(\mathrm{~d}, J_{\mathrm{H}-\mathrm{H}}=7.6 \mathrm{~Hz}, 1 \mathrm{H}\right), 7.46-7.43(\mathrm{~m}, 1 \mathrm{H}), 7.35-7.23(\mathrm{~m}, 6 \mathrm{H}), 7.18-7.15(\mathrm{~m}, 1 \mathrm{H}), 4.71-$ $4.53(\mathrm{~m}, 2 \mathrm{H}), 3.61-3.58(\mathrm{~m}, 1 \mathrm{H}), 3.47-3.44(\mathrm{~m}, 1 \mathrm{H}), 3.38(\mathrm{~s}, 3 \mathrm{H}), 2.29-2.84(\mathrm{~m}, 1 \mathrm{H}) .{ }^{19} \mathrm{~F}-\mathrm{NMR}$ $\left(470.6 \mathrm{MHz}, \mathrm{CDCl}_{3}\right) \delta:-197.63$.

\section{Preparation of compound 6a from compound 10}

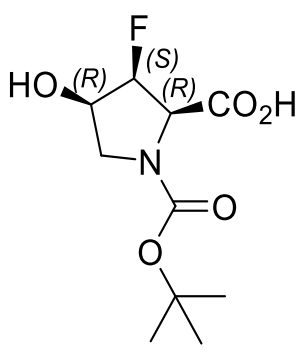

Compound $10(100 \mathrm{mg}, 0.248 \mathrm{mmol})$ was dissolved in DCM $(10 \mathrm{~mL})$. Triisopropylsilane (TIPS) $(760 \mu \mathrm{L})$ and trifluoroacetic acid (TFA) $(500 \mu \mathrm{L})$ were added, and the resultant yellow mixture was left to react at room temperature for $2 \mathrm{~h}$. Water $5 \mathrm{~mL}$ was added, the mixture was vigorously shaken and the aqueous phase was separated, washed with $2 \mathrm{~mL}$ of $\mathrm{Et}_{2} \mathrm{O}$ and freeze-dried to afford the $\mathrm{N}$ deprotected compound as trifluoroacetate salt. [ ${ }^{1} \mathrm{H}-\mathrm{NMR}\left(\mathrm{D}_{2} \mathrm{O}\right) \delta: 5.46-5.34(\mathrm{~m}, 1 \mathrm{H}), 4.83(\mathrm{dd}, J \mathrm{H}-\mathrm{F}$ $\left.=32.1 \mathrm{~Hz}, J_{\mathrm{H}-\mathrm{H}}=2.9 \mathrm{~Hz}, 1 \mathrm{H}\right), 4.70-4.61(\mathrm{~m}, 1 \mathrm{H}), 3.85(\mathrm{~s}, 3 \mathrm{H}), 3.76-3.72(\mathrm{~m}, 1 \mathrm{H}), 3.32-3.28(\mathrm{~m}, 1 \mathrm{H})$. 
${ }^{19}$ F-NMR $\left.\delta:-75.56(3 \mathrm{~F}),-208.8(1 \mathrm{~F})\right]$. The crude compound was dissolved in water $(10 \mathrm{~mL})$, cooled at $0{ }^{\circ} \mathrm{C}$, LiOH (19 mg, $\left.0.469 \mathrm{mmol}\right)$ dissolved in water $(2 \mathrm{~mL})$ was added dropwise. The mixture was stirred at $0{ }^{\circ} \mathrm{C}$ for $4 \mathrm{~h} . \mathrm{NaHCO}_{3}(21 \mathrm{mg}, 0.248 \mathrm{mmol})$ was added and the mixture was treated with ditert-butyl dicarbonate $(54 \mathrm{mg}, 0.248 \mathrm{mmol})$ dissolved in dioxane $(2 \mathrm{~mL})$. The reaction mixture was stirred overnight at r.t., cooled to $0{ }^{\circ} \mathrm{C}$ and treated with $\mathrm{KHSO}_{4}(5 \%)$ to $\mathrm{pH}=3-4$. The desired product was extracted with ethyl acetate $(3 \mathrm{X} 15 \mathrm{~mL})$ and the organic phase was dried over $\mathrm{MgSO} 4$. Obtained $40 \mathrm{mg}, 64 \%$ yield. Analytical data match those for compound $\mathbf{6 a}$.

\section{Preparation of compound 6d from compound S4}

Prepared from compound S4 (100 mg, $0.248 \mathrm{mmol})$. Obtained $33 \mathrm{mg}, 54 \%$ yield. Analytical data match those for compound $\mathbf{6 d}$.

\section{Peptide synthesis and purification}

The peptide Fmoc-TIPMDDDLRSF-Rink amide resin was synthesized in an INTAVIS RespepSL automated peptide synthesizer using solid phase Fmoc chemistry (resin loading $0.68 \mathrm{mmol} / \mathrm{g}$ ) and protocols suggested by the supplier (HATU/HBTU/NMM coupling conditions). The peptide-loaded resin (75 mg, $0.051 \mathrm{mmol}$ of peptide) was then Fmoc-deprotected and reacted with $N$-Fmoc-F-Hyp $\mathbf{S 2}$ or $\mathbf{S 3}$ (49 mg, $0.132 \mathrm{mmol}$ ), HATU (50 mg, $0.131 \mathrm{mmol})$, HOAT (18 mg, $0.131 \mathrm{mmol}$ ) and DIPEA (45 $\mu \mathrm{L}, 0.263 \mathrm{mmol}$ ) for $2 \mathrm{~h}$. After Fmoc deprotection, the peptide sequence was completed coupling the amino acids Ala, Leu, Glu(OtBu), Asp $(\mathrm{OtBu})$ and the terminal Fmoc was removed. The peptides were cleaved from the resin using a solution of TFA, water and triisopropylsilane (TIS)(92.5:2.5:5). The peptides were obtained as C-terminal amides and were purified by HPLC using a gradient from $5 \%$ of ACN (containing $0.1 \%$ of TFA) to $30 \%$ of ACN (containing $0.1 \%$ of TFA) in water (containing $0.1 \%$ of TFA) over $15 \mathrm{~min}$. The purity and identity of the peptides was determined by LC-MS using a gradient from $0 \%$ of $\mathrm{ACN}$ (containing $0.1 \%$ of TFA) to $50 \%$ of $\mathrm{ACN}$ (containing $0.1 \%$ of TFA) in water (containing $0.1 \%$ of TFA) over $20 \mathrm{~min}$.

Peptide 13a: $\mathrm{C}_{101} \mathrm{H}_{147} \mathrm{FN}_{24} \mathrm{O}_{33} \mathrm{~S}$, expected 2275.03, found $\mathrm{m} / \mathrm{z}=1138.7[\mathrm{M}+2 \mathrm{H}]^{2+}, 759.5[\mathrm{M}+3 \mathrm{H}]^{3+}$.

Peptide 13b: $\mathrm{C}_{101} \mathrm{H}_{147} \mathrm{FN}_{24} \mathrm{O}_{33} \mathrm{~S}$, expected 2275.03, found $\mathrm{m} / \mathrm{z}=1138.7[\mathrm{M}+2 \mathrm{H}]^{2+}, 759.5[\mathrm{M}+3 \mathrm{H}]^{3+}$.

\section{General procedure for the preparation of $N$-Ac-F-Hyp-OMe model compounds (12a-d)}

To a solution of compound 6a-d $(25 \mathrm{mg}, 0.100 \mathrm{mmol})$ in anhydrous $\mathrm{MeOH}(2 \mathrm{~mL})$ cooled at $0{ }^{\circ} \mathrm{C}$, trimethylsilyldiazomethane ( $2 \mathrm{M}$ in hexane) was added dropwise until the yellow colour of TMSdiazomethane persisted. The reaction mixture was left stirring at r.t. for $1 \mathrm{~h}$. Few drops of acetic acid were added to destroy the excess of trimethylsilyldiazomethane and the volatile components were removed under vacuum. The crude was dissolved in DCM $(1 \mathrm{~mL})$ and a solution of $4 \mathrm{M} \mathrm{HCl}$ in 
dioxane was added $(1 \mathrm{~mL})$. The reaction mixture was stirred at r.t. for $2 \mathrm{~h}$. Solvents were evaporated under vacuum and any excess of $\mathrm{HCl}$ was removed carefully. The solid was suspened in anhydrous DCM $(1 \mathrm{~mL})$ and TEA $(28 \mu \mathrm{L}, 0.2 \mathrm{mmol})$ was added. The reaction mixture was cooled at $0{ }^{\circ} \mathrm{C}$ and acetic anhydride $(10 \mu \mathrm{L}, 0.1 \mathrm{mmol})$ was added. The reaction mixture was stirred at r.t. for $30 \mathrm{~min}$, water $(100 \mu \mathrm{L})$ was added to quench the reaction. Volatile components were removed under reduced pressure and the crude was dissolved in methanol $(300 \mu \mathrm{L})$ and purified by preparative HPLC to obtain pure compounds (10-14 mg, 50-68\%)

\section{Methyl (2R,3S,4R)-1-acetyl-3-fluoro-4-hydroxypyrrolidine-2-carboxylate (12a)}<smiles>CC(=O)O[C@]1(F)C(F)[C@@H](O)CN1C(C)=O</smiles>

Compound 12a was obtained as a transparent glass, after HPLC purification and freeze drying. ${ }^{1} \mathrm{H}-$ NMR $\left(500 \mathrm{MHz}, \mathrm{D}_{2} \mathrm{O}\right.$; actual integration of rotamer signals is reported) $\delta: 5.50$ (ddd, $J=3.7,5.1,{ }^{1} J_{\mathrm{H}-}$ $\mathrm{F}=53.4 \mathrm{~Hz}, 0.12 \mathrm{H}), 5.49-5.36(\mathrm{~m}, 0.88 \mathrm{H}), 5.17(\mathrm{dd}, \mathrm{J}=5.5,23.4 \mathrm{~Hz}, 0.12 \mathrm{H}), 4.87$ (dd, J=4.7, 28.1 Hz, 0.88 H), 4.61 - 4.44 (m, 1H), 4.14 (dd, J=7.6, 10.2 Hz, 1H), 3.96 (s, 0.4 H), 3.91 (s, 2.6 H) 3.70 $3.66(\mathrm{~m}, 1 \mathrm{H}), 2.24(\mathrm{~s}, 2.7 \mathrm{H}), 2.1(\mathrm{~s}, 0.3 \mathrm{H}) .{ }^{19} \mathrm{~F}-\mathrm{NMR}\left(470.6 \mathrm{MHz}, \mathrm{D}_{2} \mathrm{O}\right) \delta:-205.57,-207.47 .{ }^{13} \mathrm{C}-$ $\operatorname{NMR}\left(125.8, \mathrm{D}_{2} \mathrm{O}\right) \delta: 174.2^{*}, 173.5,169\left(\mathrm{~d},{ }^{3} J_{\mathrm{C}-\mathrm{F}}=7.7 \mathrm{~Hz}\right), 92.5^{*}\left(\mathrm{~d},{ }^{1} J_{\mathrm{C}-\mathrm{F}}=187.2 \mathrm{~Hz}\right), 91.5\left(\mathrm{~d},{ }^{1} J_{\mathrm{C}-}\right.$ $\left.\mathrm{F}_{\mathrm{F}}=184.6 \mathrm{~Hz}\right), 69.1 *\left(\mathrm{~d},{ }^{2} J_{\mathrm{C}-\mathrm{F}}=17.1 \mathrm{~Hz}\right), 68.0 *\left(\mathrm{~d},{ }^{2} J_{\mathrm{C}-\mathrm{F}}=17.0 \mathrm{~Hz}\right), 62.9 *\left(\mathrm{~d},{ }^{2} J_{\mathrm{C}-\mathrm{F}}=20.1 \mathrm{~Hz}\right), 61.8$ $\left(\mathrm{d},{ }^{2} J_{\mathrm{C}-\mathrm{F}}=19.8 \mathrm{~Hz}\right), 53.7 *, 53.4,50.0,21.2,20.6 *{ }^{*} \mathrm{C}_{8} \mathrm{H}_{12} \mathrm{FNO}_{4}$, expected 205.1, found $\mathrm{m} / \mathrm{z}=206.1$, $[\mathrm{M}+\mathrm{H}]^{+}$.

\section{Methyl (2R,3R,4S)-1-acetyl-3-fluoro-4-hydroxypyrrolidine-2-carboxylate (12b)}<smiles>CC(=O)N1C[C@H](O)[C@@H](F)[C@@]1(O)C(C)=O</smiles>

Compound 12b was obtained as a transparent glass, after HPLC purification and freeze drying. ${ }^{1} \mathrm{H}-$ NMR (500 MHz, $\mathrm{D}_{2} \mathrm{O}$; actual integration of rotamer signals is reported) $\delta: 5.48-5.26(\mathrm{~m}, 1 \mathrm{H}), 5.11$ $\left(\mathrm{dd}, J_{\mathrm{H}-\mathrm{H}}=1.6 \mathrm{~Hz},{ }^{2} J_{\mathrm{H}-\mathrm{F}}=20.0 \mathrm{~Hz} 0.15 \mathrm{H}\right), 4.80\left(\mathrm{dd}, J_{\mathrm{H}-\mathrm{H}}=2.4 \mathrm{~Hz},{ }^{2} J_{\mathrm{H}-\mathrm{F}}=21.6 \mathrm{~Hz} 0.85 \mathrm{H}\right), 4.67-$ $4.58(\mathrm{~m}, 1 \mathrm{H}), 4.12(\mathrm{dd}, J=10.7,6.9 \mathrm{~Hz}, 0.85 \mathrm{~Hz}), 3.94$ (s, $0.45 \mathrm{H}), 3.89$ (s, $2.55 \mathrm{H}), 3.73-3.79$ (m, $0.85 \mathrm{H}), 3.55(\mathrm{dd}, J=11.7,7.8 \mathrm{~Hz}, 0.15 \mathrm{H}), 2.23(\mathrm{~s}, 2.5 \mathrm{H}), 2.11(\mathrm{~s}, 0.5 \mathrm{H}) .{ }^{19} \mathrm{~F}-\mathrm{NMR}(470.6 \mathrm{MHz}$, $\left.\mathrm{D}_{2} \mathrm{O}\right) \delta$ : $-199.86,-200.15^{*} .{ }^{13} \mathrm{C}-\mathrm{NMR}\left(125.8, \mathrm{D}_{2} \mathrm{O}\right) \delta: 174.2^{*}, 173.8,170.5\left(\mathrm{~d},{ }^{3} J_{\mathrm{C}-\mathrm{F}}=12.2 \mathrm{~Hz}\right)$, $170.0^{*}\left(\mathrm{~d},{ }^{3} J_{\mathrm{C}-\mathrm{F}}=13.5 \mathrm{~Hz}\right), 93.6 *\left(\mathrm{~d},{ }^{1} J_{\mathrm{C}-\mathrm{F}}=190.5 \mathrm{~Hz}\right), 92.7\left(\mathrm{~d},{ }^{1} J_{\mathrm{C}-\mathrm{F}}=190.4 \mathrm{~Hz}\right), 69.1\left(\mathrm{~d},{ }^{2} J_{\mathrm{C}-\mathrm{F}}=\right.$ 
$17.1 \mathrm{~Hz}), 68.1 *\left(\mathrm{~d},{ }^{2} J_{\mathrm{C}-\mathrm{F}}=17.1 \mathrm{~Hz}\right), 64.8^{*}\left(\mathrm{~d},{ }^{2} J_{\mathrm{C}-\mathrm{F}}=24.0 \mathrm{~Hz}\right), 63.1\left(\mathrm{~d},{ }^{2} J_{\mathrm{C}-\mathrm{F}}=25.2 \mathrm{~Hz}\right), 54.0 *, 53.6$, 20.3, 20.9, 20.4*.*Minor rotamer. $\mathrm{C}_{8} \mathrm{H}_{12} \mathrm{FNO}_{4}$, expected 205.1, found $\mathrm{m} / \mathrm{z}=206.1,[\mathrm{M}+\mathrm{H}]^{+}$.

\section{Methyl (2R,3R,4R)-1-acetyl-3-fluoro-4-hydroxypyrrolidine-2-carboxylate (12c)}

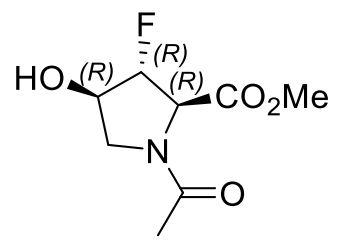

Compound 12c was obtained as a transparent glass, after HPLC purification and freeze drying. ${ }^{1} \mathrm{H}-$ NMR (500 MHz, $\left.\mathrm{D}_{2} \mathrm{O}\right) \delta: 5.48\left(\mathrm{~d},{ }^{1} J_{\mathrm{H}-\mathrm{F}}=48.0 \mathrm{~Hz}, 0.25 \mathrm{H}\right), 5.33\left(\mathrm{~d},{ }^{1} J_{\mathrm{H}-\mathrm{F}}=48.5 \mathrm{~Hz}, 0.75 \mathrm{H}\right), 5.16$ $\left(\mathrm{d},{ }^{2} J_{\mathrm{H}-\mathrm{F}}=20.3 \mathrm{~Hz}, 0.28 \mathrm{H}\right), 4.97\left(\mathrm{~d},{ }^{2} J_{\mathrm{H}-\mathrm{F}}=22.8 \mathrm{~Hz}, 0.70 \mathrm{H}\right), 4.67-4.65(\mathrm{~m}, 0.8 \mathrm{H}), 4.61-4.57$ (m, $0.3 \mathrm{H}), 4.10$ (dd, $J=4.6,11.9 \mathrm{~Hz}, 0.8 \mathrm{H}), 3.92$ (br. s, $1.1 \mathrm{H}$ ), 3.88 (br. s, $2.6 \mathrm{H}), 3.8$ (d, $J=11.9 \mathrm{~Hz}$, $0.8 \mathrm{H}), 3.7(\mathrm{~d}, J=13.2 \mathrm{~Hz}, 0.2 \mathrm{H}), 2.26(\mathrm{~s}, 2.4 \mathrm{H}), 2.19(\mathrm{~s}, 1 \mathrm{H}) .{ }^{19} \mathrm{~F}-\mathrm{NMR}\left(470.6 \mathrm{MHz}, \mathrm{D}_{2} \mathrm{O}\right) \delta$ : 183.64, -185.10*. ${ }^{13} \mathrm{C}-\mathrm{NMR}\left(125.8, \mathrm{D}_{2} \mathrm{O}\right) \delta: 174.6^{*}, 174.1,169.8^{*}, 169.7,97.2^{*}\left(\mathrm{~d},{ }^{1} J_{\mathrm{C}-\mathrm{F}}=186.7\right.$ $\mathrm{Hz}), 96.1\left(\mathrm{~d},{ }^{1} J_{\mathrm{C}-\mathrm{F}}=186.5 \mathrm{~Hz}\right), 71.8\left(\mathrm{~d},{ }^{2} J_{\mathrm{C}-\mathrm{F}}=27.4 \mathrm{~Hz}\right), 70.2 *\left(\mathrm{~d},{ }^{2} J_{\mathrm{C}-\mathrm{F}}=28.0 \mathrm{~Hz}\right), 65.0 *\left(\mathrm{~d},{ }^{2} J_{\mathrm{C}-\mathrm{F}}=\right.$ $23.4 \mathrm{~Hz}), 63.6\left(\mathrm{~d},{ }^{2} \mathrm{~J}_{\mathrm{C}-\mathrm{F}}=24.5 \mathrm{~Hz}\right), 53.6^{*}, 53.4,53.1,52.1^{*}, 21.3 . *$ Minor rotamer. $\mathrm{C}_{8} \mathrm{H}_{12} \mathrm{FNO}_{4}$, expected 205.1, found $\mathrm{m} / \mathrm{z}=206.1,[\mathrm{M}+\mathrm{H}]^{+}$.

\section{Methyl (2R,3S,4S)-1-acetyl-3-fluoro-4-hydroxypyrrolidine-2-carboxylate (12d)}

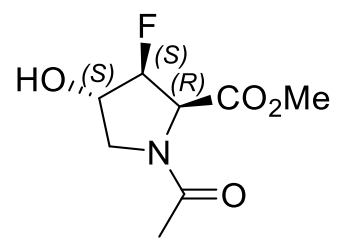

Compound 12d was obtained as a transparent glass, after HPLC purification and freeze drying. ${ }^{1} \mathrm{H}-$ NMR (500 MHz, $\left.\mathrm{D}_{2} \mathrm{O}\right) \delta: 5.54-5.33(\mathrm{~m}, 1 \mathrm{H}), 5.24\left(\mathrm{dd},{ }^{2} J_{\mathrm{H}-\mathrm{F}}=26.9 \mathrm{~Hz}, J_{\mathrm{H}-\mathrm{H}}=5.5 \mathrm{~Hz}, 0.15 \mathrm{H}\right), 4.93$ $\left(\mathrm{dd},{ }^{2} J_{\mathrm{H}-\mathrm{F}}=28.6 \mathrm{~Hz}, J_{\mathrm{H}-\mathrm{H}}=4.9 \mathrm{~Hz}, 0.85 \mathrm{H}\right), 4.66-4.59(\mathrm{~m}, 1 \mathrm{H}), 4.80-4.03(\mathrm{~m}, 1 \mathrm{H}), 3.98(\mathrm{~s}, 0.4 \mathrm{H})$, 3.92 (s, $2.6 \mathrm{H}), 2.27$ (s, $2.6 \mathrm{H}), 2.10(0.4 \mathrm{H}) .{ }^{19} \mathrm{~F}-\mathrm{NMR}\left(470.6 \mathrm{MHz}, \mathrm{D}_{2} \mathrm{O}\right) \delta:-189.76 *$, $-191.74 .{ }^{13} \mathrm{C}-$ NMR $\left(125.8, \mathrm{D}_{2} \mathrm{O}\right) \delta: 174.3^{*}, 173.6,169.7 *\left(\mathrm{~d},{ }^{3} J_{\mathrm{C}-\mathrm{F}}=9.1 \mathrm{~Hz}\right), 169.3\left(\mathrm{~d},{ }^{3} J_{\mathrm{C}-\mathrm{F}}=8.2 \mathrm{~Hz}\right), 95.8 *\left(\mathrm{~d},{ }^{1} J\right.$ C-F $=184.8 \mathrm{~Hz}), 94.2\left(\mathrm{~d},{ }^{1} J_{\mathrm{C}-\mathrm{F}}=184.5 \mathrm{~Hz}\right), 71.9\left(\mathrm{~d},{ }^{2} J_{\mathrm{C}-\mathrm{F}}=27.1 \mathrm{~Hz}\right), 70.5^{*}\left(\mathrm{~d},{ }^{2} J_{\mathrm{C}-\mathrm{F}}=27.3 \mathrm{~Hz}\right), 63.5^{*}$ $\left(\mathrm{d},{ }^{2} J_{\mathrm{C}-\mathrm{F}}=21.6 \mathrm{~Hz}\right), 62.0\left(\mathrm{~d},{ }^{2} J_{\mathrm{C}-\mathrm{F}}=20.5 \mathrm{~Hz}\right), 53.7^{*}, 53.4,53.3,53.7,53.4 *, 21.3,20.9^{*}$. *Minor rotamer. $\mathrm{C}_{8} \mathrm{H}_{12} \mathrm{FNO}_{4}$, expected 205.1, found $\mathrm{m} / \mathrm{z}=206.1$, $[\mathrm{M}+\mathrm{H}]^{+}$. 


\section{yl)benzyl)carbamoyl)pyrrolidine-1-carboxylate (S5)}<smiles>Cc1ncsc1-c1ccc(CNC(=O)[C@@H]2[C@H](F)[C@@H](O)CN2C(=O)OC(C)(C)C)cc1</smiles>

To a solution of (4-(4-methylthiazol-5-yl)phenyl)methanamine (32.7 mg, $0.160 \mathrm{mmol})$ in DMF (1 $\mathrm{mL}$ ), a mixture of N-Boc-fluoro-hydroxyproline $6 \mathbf{b}(40.0 \mathrm{mg}, 0.160 \mathrm{mmol})$, HATU (61.0 mg, 0.106 mmol), 1-hydroxy-7-azabenzotriazole (HOAT) $(22.0 \quad \mathrm{mg}, \quad 0.160 \mathrm{mmol})$ and $N, N$ Diisopropylethylamine, (DIPEA) $(85 \mu \mathrm{L}, 0.480 \mathrm{mmol})$ in DMF $(0.5 \mathrm{~mL})$ was added dropwise under stirring at r.t. After one hour, HPLC analysis showed complete conversion of the starting material and formation of the desired product (METHOD 1, $\mathrm{rt}=1.451 \mathrm{~min}, \mathrm{~m} / \mathrm{z}=436.2,[\mathrm{M}+\mathrm{H}]^{+}$). The reaction was diluted with DCM $(10 \mathrm{~mL})$, washed with water $(2 \mathrm{~mL})$, the organic phase was extracted and then dried over anhydrous $\mathrm{MgSO}_{4}$ and the solvents were then removed under vacuum. Purification by preparative HPLC (acidic method) afforded the title compound as a white solid $(60 \mathrm{mg})$ in $86 \%$ yield. ${ }^{1} \mathrm{H}-\mathrm{NMR}\left(500 \mathrm{MHz}, \mathrm{CD}_{3} \mathrm{OD}\right)$ 8: 9.10-9.07 (m, 1H), 7.47-7.46 (m, 4H), 4.97-4.85 (overlapped with signal of residual water in the solvent, $\mathrm{m}, 1 \mathrm{H}), 4.52-4.33(\mathrm{~m}, 4 \mathrm{H}), 3.81-3.76(\mathrm{~m}, 1 \mathrm{H}), 2.51(\mathrm{~s}, 3 \mathrm{H})$, $1.49^{*}(\mathrm{~s}, 9 \mathrm{H}), 1.33$ (s, 9H). ${ }^{19} \mathrm{~F}-\mathrm{NMR}\left(470.6 \mathrm{MHz}, \mathrm{CD}_{3} \mathrm{OD}\right) \delta:-198.50,-199.21^{*}$.

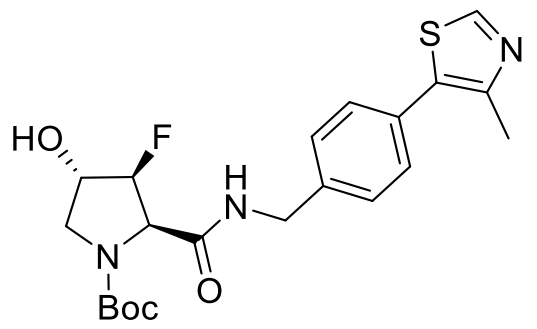

Obtained as described for preparative compound S6, and starting from compound 6d, HPLC analysis (method 2) $\mathrm{rt}=3.007 \mathrm{~min}, \mathrm{~m} / \mathrm{z}=436.2,[\mathrm{M}+\mathrm{H}]^{+} .\left(82 \%\right.$ yield, white solid). ${ }^{1} \mathrm{H}-\mathrm{NMR}\left(\mathrm{CD}_{3} \mathrm{OD}\right) \delta: 8.79$ (s, 1H), 7.48-7.44 (m, 4H), 5.12-5.01 (m, 1H), 4.63-4.39(m, 3H), 4.34-4.32 (m, 1H), 3.76-3.62 (m, 2H), $2.49(\mathrm{~s}, 3 \mathrm{H}), 1.50 *(\mathrm{~s}, 9 \mathrm{H}), 1.34(\mathrm{~s}, 9 \mathrm{H}) .{ }^{19} \mathrm{~F}-\mathrm{NMR}\left(470.6 \mathrm{MHz}, \mathrm{CD}_{3} \mathrm{OD}\right) \delta:-193.81,-194.03^{*}$ 


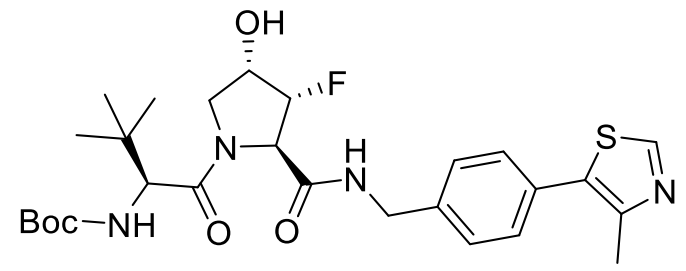

To a solution of preparative compound $\mathbf{S 5}(22.0 \mathrm{mg}, 0.05056 \mathrm{mmol})$ in DCM/MeOH 9:1 $(1.0 \mathrm{~mL})$, $\mathrm{HCl}$ in dioxane $(4 \mathrm{M}, 1.0 \mathrm{~mL})$ was added and mixture was stirred for $4 \mathrm{~h}$ at room temperature. The volatiles were removed under vacuum, and the residue was dissolved in water and freeze-dried to afford the deprotected amine as the corresponding hydrochloride salt form in quantitative yield as a light yellow powder. This salt was used without any further purification, and was suspended in DMF $(1 \mathrm{~mL})$ and DIPEA $(22 \mu \mathrm{L}, 0.126 \mathrm{mmol})$ was added. To the resulting solution, a mixture of $(S)$-2((tert-butoxycarbonyl)amino)-3,3-dimethylbutanoic acid (11.7 mg, 0,0506 mmol), HATU (19.0 mg, $0.0506 \mathrm{mmol})$, HOAT $(6.9 \mathrm{mg}, 0.05056 \mathrm{mmol})$ and DIPEA $(22 \mu \mathrm{L}, 0.126 \mathrm{mmol})$ in DMF $(0.5 \mathrm{~mL})$ was added under stirring at r.t. After $3 \mathrm{~h}$, HPLC analysis (METHOD 1, $\mathrm{rt}=1.646 \mathrm{~min}, \mathrm{~m} / \mathrm{z}=493.2$, $\left.[\mathrm{M}-\mathrm{tBu}+\mathrm{H}]^{+}\right)$showed complete conversion of the starting material and the formation of the desired product. DCM $(10 \mathrm{ml})$ was added and the mixture was washed with water $(1 \mathrm{~mL})$ and $\mathrm{NaHSO}_{4}$ solution $(5 \%, 1 \mathrm{~mL})$. The organic layer was dried over anhydrous $\mathrm{MgSO}_{4}$ and the solvents were removed under reduced pressure. The crude product was obtained as a yellow wax $(25 \mathrm{mg}, 90 \%$ yield) and was used directly in the next step without any further purification.

${ }^{1} \mathrm{H}$ NMR (500 MHz, $\left.\mathrm{CD}_{3} \mathrm{OD}\right) \quad \delta: 8.94(\mathrm{~s}, 1 \mathrm{H}), 7.48$ - $7.42(\mathrm{~m}, 4 \mathrm{H}), 5.05-4.92(\mathrm{~m}, 1 \mathrm{H}), 4.71$ - 4.63 $(\mathrm{m}, 1 \mathrm{H}), 4.59-4.47(\mathrm{~m}, 2 \mathrm{H}), 4.41-4.35(\mathrm{~m}, 1 \mathrm{H}), 4.30(\mathrm{~s}, 1 \mathrm{H}), 4.08$ - $4.03(\mathrm{~m}, 1 \mathrm{H}), 3.79-3.72(\mathrm{~m}$, $1 \mathrm{H}), 2.49(\mathrm{~s}, 3 \mathrm{H}), 1.46^{*}(\mathrm{~s}, 9 \mathrm{H}), 1.02(\mathrm{~s}, 9 \mathrm{H}) .{ }^{19} \mathrm{~F}-\mathrm{NMR}\left(470.6 \mathrm{MHz}, \mathrm{CD}_{3} \mathrm{OD}\right) \delta:-200.36$.

Tert-butyl

$((S)-1-((2 R, 3 S, 4 S)-3-f l u o r 0-4-h y d r o x y-2-((4-(4-m e t h y l t h i a z o l-5-$ yl)benzyl)carbamoyl)pyrrolidin-1-yl)-3,3-dimethyl-1-oxobutan-2-yl)carbamate (S8)

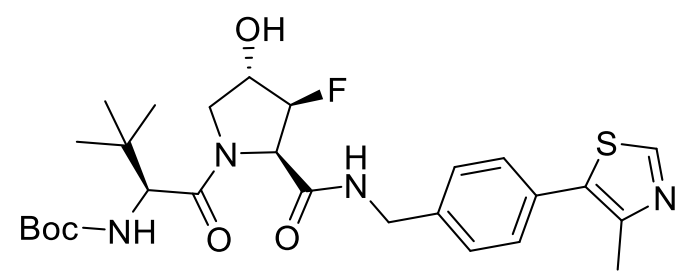

Obtained as described for compound S7, and starting from preparative compound S6. The crude product was obtained as a yellow wax in $88 \%$ yield. HPLC analysis (METHOD 1): $\mathrm{rt}=1.639 \mathrm{~min}$, 
$\mathrm{m} / \mathrm{z}=493.2,[\mathrm{M} \mathrm{M}-\mathrm{tBu}+\mathrm{H}]^{+} .{ }^{1} \mathrm{H}-\mathrm{NMR}\left(500 \mathrm{MHz}, \mathrm{CD}_{3} \mathrm{OD}\right) \delta: 8.93(\mathrm{~s}, 1 \mathrm{H}), 8.63-8.61(\mathrm{~m}, 1 \mathrm{H}), 7.47-$ $7.42(\mathrm{~m}, 4 \mathrm{H}), 5.14-5.02(\mathrm{~m}, 1 \mathrm{H}), 4.79\left(\mathrm{dd},{ }^{2} J_{\mathrm{H}-\mathrm{F}}=26.0 \mathrm{~Hz}, \mathrm{~J} \mathrm{H}-\mathrm{H}=5.3 \mathrm{~Hz}, 1 \mathrm{H}\right), 4.54-4.43(\mathrm{~m}, 3 \mathrm{H})$, $4.30(\mathrm{~s}, 1 \mathrm{H}), 3.97-3.91(\mathrm{~m}, 2 \mathrm{H}), 2.49(\mathrm{~s}, 3 \mathrm{H}), 1.44 *(\mathrm{~s}, 9 \mathrm{H}), 1.06(\mathrm{~s}, 9 \mathrm{H}) .{ }^{19} \mathrm{~F}-\mathrm{NMR}(470.6 \mathrm{MHz}$, $\left.\mathrm{CD}_{3} \mathrm{OD}\right) \delta:-193.39$.

Tert-butyl

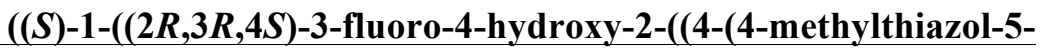
yl)benzyl)carbamoyl)pyrrolidin-1-yl)-3,3-dimethyl-1-oxobutan-2-yl)carbamate (14a)

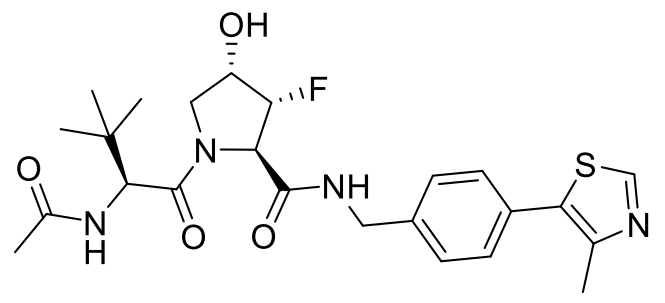

To a solution of compound $\mathbf{S} 7(25.0 \mathrm{mg}, 0.0455 \mathrm{mmol})$ in $\mathrm{DCM} / \mathrm{MeOH}$ 9:1 $(1.0 \mathrm{~mL}), \mathrm{HCl}$ in dioxane $(4 \mathrm{M}, 1.0 \mathrm{~mL})$ was added and the resultant mixture was stirred for $4 \mathrm{~h}$ at room temperature. The volatiles were removed under vacuum, the residue was dissolved in water and freeze-dried to afford the deprotected amine as a light yellow powder in quantitative yield in the form of the corresponding hydrochloride salt. This salt was used directly without any further purification and was suspended in DCM $(1 \mathrm{~mL})$, TEA $(12.7 \mu \mathrm{L}, 0.091 \mathrm{mmol})$ was added, followed by acetic anhydride $(4.3 \mu \mathrm{L}, 0.0455$ mmol) was added at $0{ }^{\circ} \mathrm{C}$, and the resultant mixture was stirred at $0{ }^{\circ} \mathrm{C}$ for two h. HPLC analysis showed complete conversion of the starting material and the formation of the desired product (method $\left.2, \mathrm{rt}=2.819 \mathrm{~min}, \mathrm{~m} / \mathrm{z}=491.2,[\mathrm{M}+\mathrm{H}]^{+}\right)$. The volatile solvents were removed and the crude was purified by preparative HPLC to obtain the title compound as a white solid (17.0 mg, $75 \%$ yield). ${ }^{1} \mathrm{H}$ NMR $\left(500 \mathrm{MHz}, \mathrm{CD}_{3} \mathrm{OD}\right) \quad \delta: 8.89(\mathrm{~s}, 1 \mathrm{H}), 7.47-7.41(\mathrm{~m}, 4 \mathrm{H}), 5.03-4.91(\mathrm{~m}, 1 \mathrm{H}), 4.65\left(\mathrm{dd}, J_{\mathrm{H}-\mathrm{F}}=\right.$ $\left.21.4 \mathrm{~Hz}, J_{\mathrm{H}-\mathrm{H}}=2.8 \mathrm{~Hz}, 1 \mathrm{H}\right), 4.60(\mathrm{~s}, 1 \mathrm{H}), 4.58-4.35(\mathrm{~m}, 3 \mathrm{H}), 4.06-4.03(\mathrm{~m}, 1 \mathrm{H}), 3.79-3.75(\mathrm{~m}, 1 \mathrm{H})$, $2.48(\mathrm{~s}, 3 \mathrm{H}), 2.01(\mathrm{~s}, 3 \mathrm{H}), 1.04(\mathrm{~s}, 9 \mathrm{H}) .{ }^{19} \mathrm{~F}-\mathrm{NMR}\left(470.6 \mathrm{MHz}, \mathrm{CD}_{3} \mathrm{OD}\right) \delta:-199.02 *,-200.3 .{ }^{13} \mathrm{C}-$ NMR (125.8, $\left.\mathrm{CD}_{3} \mathrm{OD}\right) \delta: 171.9,171.2,169.0\left(\mathrm{~d},{ }^{3} J_{\mathrm{C}-\mathrm{F}}=9.4 \mathrm{~Hz}\right), 151.5,147.7,138.6,131.9,130.2$, 129.2, 128.1, 127.5, 93.0 (d, $\left.{ }^{1} J=190.3 \mathrm{~Hz}\right), 69.7\left(\mathrm{~d},{ }^{2} J=16.9 \mathrm{~Hz}\right), 64.4\left(\mathrm{~d},{ }^{2} J=23.9 \mathrm{~Hz}\right), 57.2,50.7$, 42.4, 34.7, 25.5, 20.8, 14.4. HRMS: $\mathrm{C}_{24} \mathrm{H}_{31} \mathrm{FN}_{4} \mathrm{O}_{4} \mathrm{~S}$, expected 490.2050, found $\mathrm{m} / \mathrm{z}=491.2072$ $[\mathrm{M}+\mathrm{H}]^{+}$. 


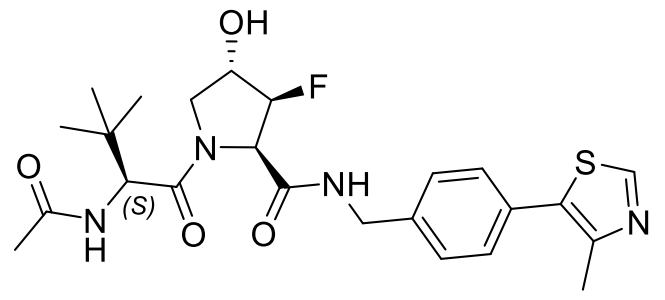

Obtained as described for compound 14a, and starting from compound S8. The product was obtained in $76 \%$ yield as a white solid. HPLC analysis (METHOD 2) $\mathrm{rt}=2.901 \mathrm{~min}, \mathrm{~m} / \mathrm{z}=491.2,[\mathrm{M}+\mathrm{H}]^{+}$. ${ }^{1} \mathrm{H}$ NMR $\left(500 \mathrm{MHz}, \mathrm{CD}_{3} \mathrm{OD}\right) \quad \delta: 8.88(\mathrm{~s}, 1 \mathrm{H}), 7.47-7.41(\mathrm{~m}, 4 \mathrm{H}), 5.14-5.00(\mathrm{~m}, 1 \mathrm{H}), 4.77\left(\mathrm{dd}, J_{\mathrm{H}-\mathrm{F}}=\right.$ $\left.26.2 \mathrm{~Hz}, J_{\mathrm{H}-\mathrm{H}}=5.2 \mathrm{~Hz}, 1 \mathrm{H}\right), 4.63(\mathrm{~s}, 1 \mathrm{H}), 4.55-4.32(\mathrm{~m}, 3 \mathrm{H}), 4.00-3.91(\mathrm{~m}, 2 \mathrm{H}), 2.48(\mathrm{~s}, 3 \mathrm{H}), 2.01(\mathrm{~s}$, $3 \mathrm{H}), 1.08(\mathrm{~s}, 9 \mathrm{H}) .{ }^{19} \mathrm{~F}-\mathrm{NMR}\left(470.6 \mathrm{MHz}, \mathrm{CD}_{3} \mathrm{OD}\right) \delta:-193.12 .{ }^{13} \mathrm{C}-\mathrm{NMR}\left(125.8, \mathrm{CD}_{3} \mathrm{OD}\right) \delta: 171.8$, $171.3,167.0\left(\mathrm{~d},{ }^{3} J_{\mathrm{C}-\mathrm{F}}=6.4 \mathrm{~Hz}\right), 154.4,147.7,138.7,132.0,130.2,129.0,127.9,127.6,94.2\left(\mathrm{~d},{ }^{1} J=\right.$ $186.2 \mathrm{~Hz}), 72.1\left(\mathrm{~d},{ }^{2} J=26.4 \mathrm{~Hz}\right), 62.9\left(\mathrm{~d},{ }^{2} J=21.3 \mathrm{~Hz}\right), 57.8,53.4,42.3,34.9,25.6,20.6,14.4$. HRMS: $\mathrm{C}_{24} \mathrm{H}_{31} \mathrm{FN}_{4} \mathrm{O}_{4} \mathrm{~S}$, expected 490.2050, found $\mathrm{m} / \mathrm{z}=491.2063[\mathrm{M}+\mathrm{H}]^{+}$.

$(2 R, 3 S, 4 S)-1-((S)-2-(t e r t-b u t y l)-17-((S)-4-(4-c h l o r o p h e n y l)-2,3,9-t r i m e t h y l-6 H-t h i e n o[3,2-$<smiles>Cc1ncsc1-c1ccc(CNC(=O)[C@@H]2[C@@H](F)[C@@H](O)CN2C(=O)[C@H](NC(=O)COCCOCCOCCNC(=O)C[C@H]2N=C(c3ccc(Cl)cc3)c3c(sc(C)c3C)-n3c(C)nnc32)C(C)(C)C)cc1</smiles>

To a solution of compound $\mathbf{S 8}(15 \mathrm{mg}, 0.034 \mathrm{mmol})$ in DCM $(0.5 \mathrm{~mL}), \mathrm{HCl}$ in dioxane $(4 \mathrm{M}, 0.5 \mathrm{~mL})$ was added. Methanol $(0.2 \mathrm{~mL})$ was added to solubilize the precipitate that formed after few min. After $2 \mathrm{~h}$, HPLC analysis showed complete conversion of the starting material and formation of the desired free amine (METHOD $2 \mathrm{rt}=2.403 \mathrm{~min}, \mathrm{~m} / \mathrm{z}=449.2,[\mathrm{M}+\mathrm{H}]^{+}$). The volatile components were removed under reduced pressure and the crude product was suspended in DMF $(1 \mathrm{~mL})$. DIPEA $(10 \mu \mathrm{L}, \quad 0.051 \mathrm{mmol})$ was added, followed by a mixture of 2-(2-(2-(2- 
azidoethoxy)ethoxy)ethoxy)acetic acid ( $8.0 \mathrm{mg}, 0.034 \mathrm{mmol})$, HATU (13.0 mg, $0.034 \mathrm{mmol})$, HOAT $(4.6 \mathrm{mg}, 0.034 \mathrm{mmol})$ and DIPEA $(10 \mu \mathrm{L}, 0.051 \mathrm{mmol})$. The resulting mixture was stirred at room temperature for one hour. HPLC analysis showed complete conversion of the starting material and formation of the desired product (METHOD $2 \mathrm{rt}=3.241 \mathrm{~min}, \mathrm{~m} / \mathrm{z}=664.3,[\mathrm{M}+\mathrm{H}]^{+}$). The crude mixture was diluted with EtOAc $(10 \mathrm{~mL})$, washed with brine $(2 \mathrm{~mL})$ and solvents were removed under reduced pressure. The crude product was purified by preparative HPLC to obtain the desired compound as transparent oil (11.2mg, 50 \%yield). To a solution of the azide ( $7.0 \mathrm{mg}, 0.0105 \mathrm{mmol})$ in methanol $(2.0 \mathrm{~mL})$, a catalytic amount of $\mathrm{Pd} / \mathrm{C}(10 \%$ dry $)$ was added. The mixture was stirred under hydrogen atmosphere for one hour. HPLC analysis showed complete conversion of the starting material and formation of the desired intermediate amine (Method 2, $\mathrm{rt}=2.674 \mathrm{~min}, \mathrm{~m} / \mathrm{z}=638.3$, $[\mathrm{M}+\mathrm{H}]^{+}$). The mixture was filtered through a syringe filter and the solvent was removed. The crude amine intermediate was then dissolved in DMF $(0.5 \mathrm{~mL})$, and added to a solution of JQ1-COOH (4.2 $\mathrm{mg}, 0.0105 \mathrm{mmol})$, HATU (4.0 mg, $0.0105 \mathrm{mmol})$, HOAT (1.5 mg, $0.0105 \mathrm{mmol})$ and DIEPA (5.4 $\mu \mathrm{L}, 0.0315 \mathrm{mmol})$ in DMF $(0.05 \mathrm{~mL})$. The resultant mixture was stirred at room temperature for $3 \mathrm{~h}$. HPLC analysis (method 2, $\mathrm{rt}=3.532 \mathrm{~min}, \mathrm{~m} / \mathrm{z}=1020.3,[\mathrm{M}+\mathrm{H}]^{+}$) showed complete conversion of the starting material and formation of the desired PROTAC product. Purification by preparative HPLC afforded the pure product, $7.5 \mathrm{mg}, 70 \%$ yield based on the amount of JQ1-COOH reacted, as white solid.

${ }^{1} \mathrm{H}$ NMR (500 MHz, CD $\left.{ }_{3} \mathrm{OD}\right) \delta: 8.93(\mathrm{~s}, 1 \mathrm{H}), 7.48$ - $7.39(\mathrm{~m}, 8 \mathrm{H}), 5.15$ - $4.99(\mathrm{~m}, 1 \mathrm{H}), 4.83$ - $4.72(\mathrm{~m}$, 2H), 4.67 - $4.63(\mathrm{~m}, 1 \mathrm{H}), 4.51$ - $4.41(\mathrm{~m}, 3 \mathrm{H}), 4.12$ - $4.03(\mathrm{~m}, 2 \mathrm{H}), 3.96$ - $3.93(\mathrm{~m}, 2 \mathrm{H}), 3.74$ - $3.59(\mathrm{~m}$, $11 \mathrm{H}), 3.49-3.43(\mathrm{~m}, 3 \mathrm{H}), 2.71(\mathrm{~s}, 3 \mathrm{H}), 2.48(\mathrm{~s}, 6 \mathrm{H}), 2.46(\mathrm{~s}, 6 \mathrm{H}), 1.71(\mathrm{~s}, 3 \mathrm{H}), 1.08(\mathrm{~s}, 9 \mathrm{H}) .{ }^{19} \mathrm{~F}-\mathrm{NMR}$ (470.6 MHz, $\left.\mathrm{CD}_{3} \mathrm{OD}\right) \delta:-195.32 .{ }^{13} \mathrm{C}-\mathrm{NMR}\left(125.8, \mathrm{CD}_{3} \mathrm{OD}\right) \delta: 170.8,170.6,165.0,155.6,151.7$, $150.9,138.9,136.8,136.4,132.2,132.1,130.8,130.6,130.1,129.9,128.9,128.4,127.6,95.1,93.3$, 72.3, 72.0, 70.9, 70.3, 70.2, 70.0, 69.7, 69.3, 63.1, 62.9, 56.7, 53.6, 48.2, 42.4, 39.2, 37.1, 35.6, 25.6, 14.2. HRMS: $\mathrm{C}_{49} \mathrm{H}_{59} \mathrm{ClFN}_{9} \mathrm{O}_{8} \mathrm{~S}_{2}$, expected 1019.3601, found $\mathrm{m} / \mathrm{z}=1020.3642[\mathrm{M}+\mathrm{H}]^{+}$. 


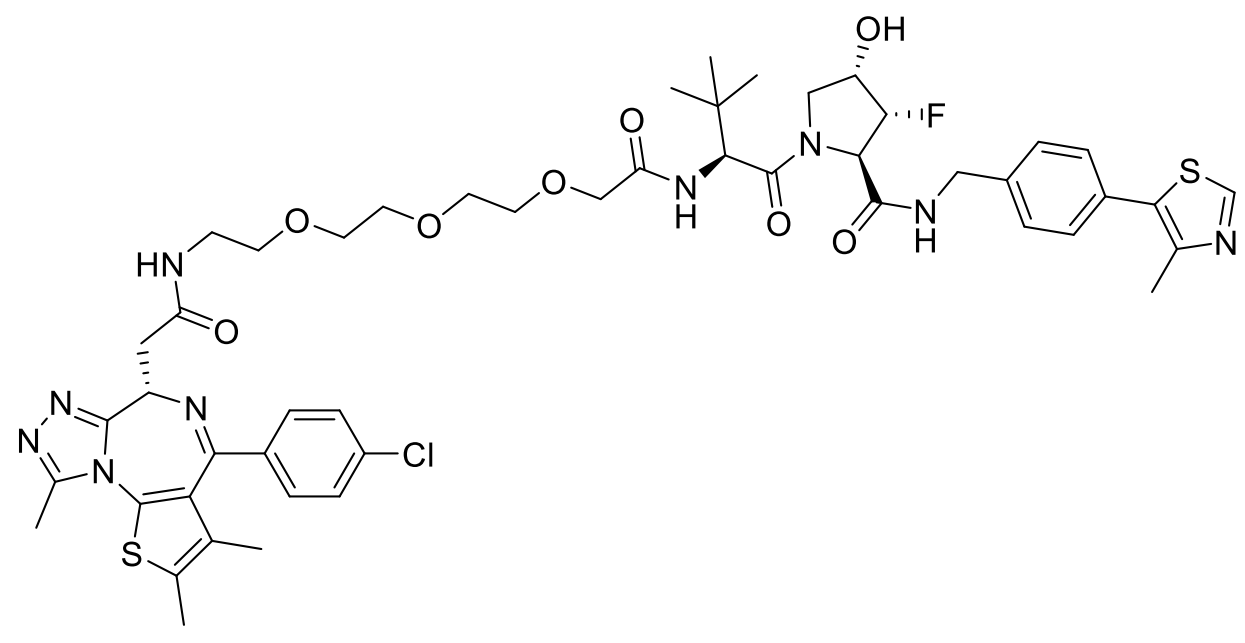

Prepared as described for preparative compound $\mathbf{1 5 b}$, starting from compound $\mathbf{S 7}$. Obtained $5.5 \mathrm{mg}$, $72 \%$ yield based on the amount of JQ1-COOH, white solid. HPLC analysis (method 2, $\mathrm{rt}=3.554 \mathrm{~min}$, $\left.\mathrm{m} / \mathrm{z}=1020.3,[\mathrm{M}+\mathrm{H}]^{+}\right)$

${ }^{1} \mathrm{H}$ NMR (500 MHz, $\left.\mathrm{CD}_{3} \mathrm{OD}\right) \quad \delta: 8.88(\mathrm{~s}, 31367398 \mathrm{H}), 7.48-7.39(\mathrm{~m}, 9 \mathrm{H}), 5.06-4.90(\mathrm{~m}, 1 \mathrm{H}), 4.71$ - $4.68(\mathrm{~m}, 1 \mathrm{H}), 4.66$ - $4.48(\mathrm{~m}, 4 \mathrm{H}), 4.36(\mathrm{~d}, \mathrm{~J}=16.1 \mathrm{~Hz}, 1 \mathrm{H}), 4.10$ - $4.05(\mathrm{~m}, 3 \mathrm{H}), 3.77$ - 3.66 (m, $10 \mathrm{H}), 3.61(\mathrm{t}, \mathrm{J}=5.8 \mathrm{~Hz}, 2 \mathrm{H}), 3.49-3.43(\mathrm{~m}, 3 \mathrm{H}), 2.70(\mathrm{~s}, 3 \mathrm{H}), 2.48(\mathrm{~s}, 3 \mathrm{H}), 2.46(\mathrm{~s}, 3 \mathrm{H}), 1.71(\mathrm{~s}, 3 \mathrm{H})$, 1.06 (s, 9H). ${ }^{19} \mathrm{~F}-\mathrm{NMR}\left(470.6 \mathrm{MHz}, \mathrm{CD}_{3} \mathrm{OD}\right) \delta:-200.23 .{ }^{13} \mathrm{C}-\mathrm{NMR}\left(125.8, \mathrm{CD}_{3} \mathrm{OD}\right) \delta: 171.9,171.2$, $169.3,169.2,151.5,147.7,138.6,132.0,130.2$, 129.2, 129.0, 128.1, 127.5, 94.0, 92.1, 69.8, 69.6, 64.5, 64.2, 57.2, 56.4, 50.8, 49.1, 42.4, 34.7, 25.5, 20.8, 14.4. $\mathrm{C}_{49} \mathrm{H}_{59} \mathrm{ClFN}_{9} \mathrm{O}_{8} \mathrm{~S}_{2}$, expected 1019.3601, found $\mathrm{m} / \mathrm{z}=1020.3614[\mathrm{M}+\mathrm{H}]^{+}$. 


\section{Computational methods}

F-Hyps 12a-d and reference compounds Ac-Hyp-OMe, Ac-hyp-OMe, Ac-3-Fpro-OMe, Ac-3-fpro$\mathrm{OMe}$ and Ac-Pro-OMe

All-atom minimizations of the trans $\mathrm{C}^{4}$ endo and exo puckers of all compounds were carried out using density functional theory (DFT) at the MN15-L/aug-cc-pVTZ(-F) level of theory in Jaguar 9.4 (Schrödinger Inc., LLC), and a tight self-consistent field (SCF) convergence criterion was imposed. The effects of water solvation were implicitly considered using the finite-element Poisson Boltzmann Solver $(\mathrm{PBF})^{4}$ version 4.1 implemented in Jaguar. Frequency calculations of the optimized structures yielded no imaginary or low $\left(>-10 \mathrm{~cm}^{-1}\right)$ frequencies, indicating a true stationary point on the potential energy surface has been attained. The resulting SCF energies were corrected by the zeropoint vibrational energy (ZPE) determined in the frequency calculations, and are listed in Supp. Table 2. Optimized geometries were subjected to orbital energy analysis by natural bond order (NBO) version $6.0^{5}$ implemented in Jaguar at the same level of theory, and weighted values of $n \rightarrow \pi^{*}$ stabilization energies were estimated according to the predicted relative abundance of each conformer. Topological analysis of the electron density by non-covalent interactions $(\mathrm{NCI})^{6}$ was performed in Jaguar, whereas atoms-in-molecules (AIM) ${ }^{7}$ inspection was performed in Multiwfn ${ }^{8}$ using wave functions generated by Jaguar at the same level of theory. The electrostatic potential $V_{\alpha}(r)^{9}$ evaluated at a distance of $0.55 \AA$ from the hydroxyl hydrogen atom along the $\mathrm{O}-\mathrm{H}$ bond, was calculated from a single-point energy (SPE) calculation of the optimized compounds using Jaguar.

\section{VHL ligands VH032, $14 \boldsymbol{a}$ and $14 \boldsymbol{b}$ in complex with $V H L$}

The crystal structures of VBC in complex with VHO32 (PDB 4W9H, ref. ${ }^{10}$ ), 14a and 14b were used for the molecular modeling. Elongins $\mathrm{B}$ and $\mathrm{C}$ and all water molecules except for the one buried in VHL and directly involved in binding to the acetyl tail were removed. Then, the complexes were prepared using the Protein Preparation Wizard (Schrödinger Inc.). Amino acid protonation states were assigned using PROPKA 3.0 (refs. ${ }^{11,12}$ ). The prepared systems were subjected to quantum mechanics/molecular mechanics (QM/MM) single-point energy (SPE) calculations in water solvation continuum using Qsite (Schrödinger, LLC). Small molecules were treated using DFT at the MN15L/aug-cc-pVTZ(-F) level of theory and the OPLS 2005 force field was used for all other atoms. Molecular electrostatic potential (MEP) surfaces of each ligand were generated in Maestro (Schrödinger, LLC). The prepared systems were also subjected to relative interaction energy estimation using the molecular mechanics/Generalized-Born Surface Area (MM-GBSA) protocol in Prime 3.0 (Schrödinger Inc.), considering water solvation and a protein shell of $5.0 \AA$ surrounding the compounds as flexible with constraints. 


\section{Small molecules Crystal structures}

Intensity data for $4 \mathbf{a}-\mathbf{c}$ were collected at $100 \mathrm{~K}$ using $\mathrm{Cu} \mathrm{K \alpha}$ radiation $(\lambda=1.54184 \AA)$; data for DAT27 were collected at the same temperature using Mo K $\alpha$ radiation $(\lambda=0.71073 \AA)$. The structures were solved without difficulty using direct methods and the structural models were optimised and developed by refinement against $|F|^{2}$ with SHELXL-2014 (ref. ${ }^{13}$ ). The structures were checked and verified with PLATON (ref. ${ }^{14}$ ) and the molecular graphics were drawn with ORTEP ${ }^{15}$. Full details of the refinements including atomic positional parameters and all geometrical data are available in the deposited cifs.

The absolute structure of $\mathbf{4 c}$ is well defined based on the refined value of the Flack absolute structure parameter ${ }^{16}$ with $\mathrm{C} 2, \mathrm{C} 3$ and $\mathrm{C} 4$ each having an $R$ configuration. This is reflected in the $\mathrm{C} 1-\mathrm{C} 2-\mathrm{C} 3-$ F1 and F1-C3-C4-O3 torsion angles of 157.94 (12) and -169.51 (12) ${ }^{\circ}$, respectively. The PLATON geometrical analysis indicated that the conformation of the $\mathrm{C} 2 / \mathrm{C} 3 / \mathrm{C} 4 / \mathrm{C} 5 / \mathrm{N} 1$ ring is best described as twisted about the $\mathrm{C} 3-\mathrm{C} 4$ bond [deviation of $\mathrm{C} 3$ and $\mathrm{C} 4$ from $\mathrm{C} 2 / \mathrm{N} 1 / \mathrm{C} 5=0.421$ (4) and -0.183 (4) $\AA$, respectively] although it is close to an envelope with $\mathrm{C} 3$ as the flap [deviation of $\mathrm{C} 3=0.567$ (2) $\AA$ from the other four atoms (r.m.s. deviation $=0.032 \AA$ )]. The dihedral angle between the fivemembered ring (all atoms) and the $\mathrm{C} 12-\mathrm{C} 17$ benzene ring is $47.30(6)^{\circ}$. The dihedral angles between the five-membered ring and the $\mathrm{C} 1 / \mathrm{O} 1 / \mathrm{O} 2$ and $\mathrm{C} 6 / \mathrm{O} 4 / \mathrm{O} 5$ groups are $70.61(18)^{\circ}$ and $13.07(15)^{\circ}$, respectively. In the crystal of $\mathbf{4 c}$, the molecules are linked into [010] chains via $\mathrm{O} 3-\mathrm{H} 1 \mathrm{o} \Lambda \mathrm{O} 5$ hydrogen bonds $[\mathrm{H} \Lambda \mathrm{O}=1.80$ (2) $\AA]$ Supp. Figure 3) with adjacent molecules in the chain related by the $2_{1}$ screw axis.

The absolute configurations of the chiral centres in $\mathbf{4 b}$ are $\mathrm{C} 2 R, \mathrm{C} 3 R$ and $\mathrm{C} 4 \mathrm{~S}$; the $\mathrm{C} 1-\mathrm{C} 2-\mathrm{C} 3-\mathrm{F} 1$ and $\mathrm{F} 1-\mathrm{C} 3-\mathrm{C} 4-\mathrm{O} 3$ torsion angles are $158.04(12)^{\circ}$ and $-51.65(18)^{\circ}$, respectively. Here, the fivemembered ring is clearly twisted on $\mathrm{C} 3-\mathrm{C} 4$ [deviation of $\mathrm{C} 3$ and $\mathrm{C} 4$ from $\mathrm{C} 2 / \mathrm{N} 1 / \mathrm{C} 5=0.308$ (4) and -0.329 (4) A, respectively]. The dihedral angles between the five-membered ring and the pendant benzene ring, the $\mathrm{C} 1 / \mathrm{O} 1 / \mathrm{O} 2$ and $\mathrm{C} 6 / \mathrm{O} 4 / \mathrm{O} 5$ groups are $78.08(6)^{\circ}, 85.73(14)^{\circ}$ and $13.2(2)^{\circ}$, respectively. In the crystal, the molecules are linked into [100] chains via $\mathrm{O} 3-\mathrm{H} 1 \mathrm{o} \Lambda \mathrm{O} 5$ hydrogen bonds $[\mathrm{H} \Lambda \mathrm{O}=1.93$ (2) $\AA]$ (Supp. Figure 4) with adjacent molecules in the chain related by the $2_{1}$ screw axis.

The absolute configurations of the chiral centres in $\mathbf{4 a}$, which crystallised as a monohydrate (Fig. 3), are $\mathrm{C} 2 R, \mathrm{C} 3 \mathrm{~S}$ and $\mathrm{C} 4 R$. The $\mathrm{C} 1-\mathrm{C} 2-\mathrm{C} 3-\mathrm{F} 1$ and $\mathrm{F} 1-\mathrm{C} 3-\mathrm{C} 4-\mathrm{O} 3$ torsion angles are $-31.09(16)^{\circ}$ and $43.15(16)^{\circ}$, respectively. The closest pucker descriptor for the five-membered ring is twisted on $\mathrm{C} 3-$ $\mathrm{C} 4$ [deviations of $\mathrm{C} 3$ and $\mathrm{C} 4=0.165$ (4) $\AA$ and -0.449 (4) $\AA$, respectively]. The alternative envelope description has $\mathrm{C} 4$ deviating by -0.581 (3) $\AA$ from the other atoms (r.m.s. deviation $=0.028 \AA$ ); note that $\mathrm{C} 3$ was the flap atom in $\mathbf{4 c}$. With respect to the benzene ring, the $\mathrm{C} 1 / \mathrm{O} 1 / \mathrm{O} 2$ and $\mathrm{C} 6 / \mathrm{O} 4 / \mathrm{O} 5$ groups, the five-membered ring subtends dihedral angles of $83.72(6)^{\circ}, 59.73(12)^{\circ}$ and $14.34(15)^{\circ}$, 
respectively. The extended structure of $\mathbf{4 a}$ features $\mathrm{O}-\mathrm{H} \Lambda \mathrm{O}$ hydrogen bonds involving both organic molecule and water molecule, which generate [010] chains (Supp. Figure 5).

The stereogenic centres in $\mathbf{1 0}$ were assigned as $\mathrm{C} 2 R, \mathrm{C} 3 S, \mathrm{C} 4 R$ on the basis of unchanging chiral centres in the synthesis. The $\mathrm{C} 1-\mathrm{C} 2-\mathrm{C} 3-\mathrm{F} 1$ and $\mathrm{F} 1-\mathrm{C} 3-\mathrm{C} 4-\mathrm{O} 3$ torsion angles are $19.2(2)^{\circ}$ and -40.0 $(2)^{\circ}$, respectively. These are distinctly different to the equivalent values in $\mathbf{4 a}$, which might in part be due to the formation of an intramolecular $\mathrm{O} 3-\mathrm{H} 1 \mathrm{o} \Lambda \mathrm{O} 2$ hydrogen bond in this structure $[\mathrm{H} \Lambda \mathrm{O}=2.28$ (3) $\AA ;$; $\left.\mathrm{O}-\mathrm{H} \Lambda \mathrm{O}=145(3)^{\circ}\right]$. The conformation of the five-membered ring is also different to 4a, being twisted on $\mathrm{C} 4-\mathrm{C} 5$ [deviation of $\mathrm{C} 4$ and $\mathrm{C} 5$ from $\mathrm{N} 1 / \mathrm{C} 2 / \mathrm{C} 3=-0.399$ (5) $\AA$ and 0.371 (5) $\AA$, respectively]. The dihedral angles between the five-membered ring (all atoms) and the C1/O1/O2 group, the $\mathrm{C} 8-\mathrm{C} 13$ benzene ring and the $\mathrm{C} 14-\mathrm{C} 25$ biphenyl unit are $88.00(15)^{\circ}, 52.52(8)^{\circ}$ and 89.13 $(6)^{\circ}$, respectively; the dihedral angle between $\mathrm{C} 8-\mathrm{C} 13$ and $\mathrm{C} 14-\mathrm{C} 25$ is $81.14(6)^{\circ}$.

\section{Crystal data}

Compound $4 \mathbf{c} \mathrm{C}_{17} \mathrm{H}_{22} \mathrm{FNO}_{5}, M_{\mathrm{r}}=339.35, \mathrm{Cu} \mathrm{K} \alpha$ radiation, $\lambda=1.54184 \AA, T=100 \mathrm{~K}$, colourless block, orthorhombic, space group $P 2{ }_{1} 2_{1} 2_{1}$ (No. 19), $Z=4, a=8.10217$ (6) $\AA, b=12.10152$ (10) $\AA, c$ $=17.38923$ (14) $\AA, V=1704.99$ (2) $\AA^{3}$. Number of measured and unique reflections $=24039$ and 3086 , respectively $\left(-9 \leq h \leq 9,-13 \leq k \leq 14,-20 \leq l \leq 20 ; 2 \theta_{\max }=136.5^{\circ} ; R_{\text {int }}=0.027\right)$. Flack absolute structure parameter $=-0.02(5)$. Final $R(F)=0.023, w R\left(F^{2}\right)=0.057$ for 223 parameters and 3053 reflections with $I>2 \sigma(I)$ (corresponding $R$-values based on all 3086 reflections $=0.023$ and 0.057, respectively), CCDC deposition number 1828831.

Compound $4 \mathbf{b} \mathrm{C}_{17} \mathrm{H}_{22} \mathrm{FNO}_{5}, M_{\mathrm{r}}=339.35, \mathrm{Cu} \mathrm{K \alpha}$ radiation, $\lambda=1.54184 \AA, T=100 \mathrm{~K}$, colourless block, orthorhombic, space group $P 2{ }_{1} 2_{1} 2_{1}$ (No. 19), $Z=4, a=8.61245$ (6) $\AA, b=10.21862$ (6) $\AA, c=$ 18.99958 (12) $\AA, V=1672.102$ (17) $\AA^{3}$. Number of measured and unique reflections $=29237$ and 2974 , respectively $\left(-10 \leq h \leq 10,-12 \leq k \leq 12,-21 \leq l \leq 22 ; 2 \theta_{\max }=136.4^{\circ} ; R_{\text {int }}=0.020\right)$. Flack absolute structure parameter $=0.01(2)$. Final $R(F)=0.024, w R\left(F^{2}\right)=0.057$ for 224 parameters and 2973 reflections with $I>2 \sigma(I)$ (corresponding $R$-values based on all 2974 reflections $=0.024$ and 0.057 , respectively), CCDC deposition number 1828832.

Compound 4a $\mathrm{C}_{17} \mathrm{H}_{22} \mathrm{FNO}_{5} \cdot \mathrm{H}_{2} \mathrm{O}, M_{\mathrm{r}}=357.37, \mathrm{Cu} \mathrm{K \alpha}$ radiation, $\lambda=1.54184 \AA, T=100 \mathrm{~K}$, colourless block, orthorhombic, space group $P 2{ }_{1} 2_{1} 2_{1}$ (No. 19), $Z=4, a=6.05736$ (4) $\AA, b=8.48378$ (6) $\AA, c=$ 34.6061 (3) $\AA, V=1778.38$ (2) $\AA^{3}$. Number of measured and unique reflections $=28685$ and 3220 , respectively $\left(-7 \leq h \leq 7,-9 \leq k \leq 10,-40 \leq l \leq 41 ; 2 \theta_{\max }=136.5^{\circ} ; R_{\text {int }}=0.018\right)$. Flack absolute structure parameter $=0.03(2)$. Final $R(F)=0.022, w R\left(F^{2}\right)=0.054$ for 239 parameters and 3217 reflections with $I>2 \sigma(I)$ (corresponding $R$-values based on all 3220 reflections $=0.022$ and 0.054 , respectively), CCDC deposition number 1828833.

Compound $10 \mathrm{C}_{25} \mathrm{H}_{22} \mathrm{FNO}_{3}, M_{\mathrm{r}}=403.43$, Mo $\mathrm{K} \alpha$ radiation, $\lambda=0.71073 \AA, T=100 \mathrm{~K}$, colourless block, monoclinic, space group $P 2_{1}$ (No. 4), $Z=2, a=9.9574$ (7) $\AA, b=8.8817$ (7) $\AA, c=10.7882$ 
(9) $\AA, \beta=90.883(7)^{\circ}, V=953.99$ (13) $\AA^{3}$. Number of measured and unique reflections $=12291$ and 4067 , respectively $\left(-12 \leq h \leq 12,-11 \leq k \leq 11,-13 \leq l \leq 14 ; 2 \theta_{\max }=54.9^{\circ} ; R_{\text {int }}=0.026\right)$. Absolute structure (C2 R, C3 $S, \mathrm{C} 4 R$ ) assumed on the basis of unchanging stereogenic centres in the synthesis. Final $R(F)=0.033, w R\left(F^{2}\right)=0.085$ for 275 parameters and 3901 reflections with $I>2 \sigma(I)$ (corresponding $R$-values based on all 4067 reflections $=0.035$ and 0.086, respectively), CCDC deposition number 1828834 .

\section{Protein expression and purification.}

Wild-type versions of human proteins VHL (UniProt accession number: P40337), ElonginC (Q15369), ElonginB (Q15370), Brd2 ( $\underline{\mathrm{P} 25440)}$ ) and $\mathrm{Brd4}(\underline{\mathrm{O} 60885})$ were used for all protein expression. For expression of VBC, N-terminally His 6 -tagged VHL (54-213), ElonginC (17-112) and ElonginB (1-104) were co-expressed in Escherichia coli BL21(DE3) at $24{ }^{\circ} \mathrm{C}$ for $16 \mathrm{~h}$ using $0.3 \mathrm{mM}$ isopropyl $\beta$-D-1-thiogalactopyranoside (IPTG). E. coli cells were lysed using a pressure cell homogenizer (Stansted Fluid Power) and lysate clarified by centrifugation. His ${ }_{6}$-tagged VCB was purified on a HisTrap FF affinity column (GE Healthcare) by elution with an imidazole gradient. The His $_{6}$ tag was removed using TEV protease and the untagged complex dialyzed into low-concentration imidazole buffer. VCB was then flowed through the HisTrap FF column a second time, allowing impurities to bind, as the complex eluted without binding. VCB was then additionally purified by anion exchange and size-exclusion chromatography using ResourceQ and Superdex-75 columns (GE Healthcare), respectively. The final purified complex was stored in $20 \mathrm{mM}$ Bis-Tris, $\mathrm{pH} 7,150 \mathrm{mM}$ sodium chloride and $1 \mathrm{mM}$ dithiothreitol (DTT). $\operatorname{Brd}^{\mathrm{BD} 1}(71-194)$, and $\mathrm{Brd}^{\mathrm{BD} 2}(333-460)$ were expressed with an N-terminal His 6 tag in E. coli BL21(DE3) at $18{ }^{\circ} \mathrm{C}$ for $20 \mathrm{~h}$ using $0.2 \mathrm{mM}$ IPTG. His $_{6}$-tagged BDs were purified on nickel Sepharose 6 fast flow beads (GE Healthcare) by elution with increasing concentrations of imidazole. For crystallography the His ${ }_{6}$-tagged BD was cleaved with TEV protease and dialyzed into low-concentration imidazole buffer. The BD was then flowed over the nickel beads a second time to remove impurities and protease. BDs were then additionally purified by size-exclusion chromatography using a Superdex-75 column. The BDs were purified by sizeexclusion chromatography using a Superdex-75 column. The final purified proteins were stored in 20 mM 4-(2-hydroxyethyl)-1-piperazineethanesulfonic acid (HEPES), pH 7.5, $150 \mathrm{mM}$ sodium chloride and $1 \mathrm{mM}$ DTT. All chromatography purification steps were performed using Äkta FPLC purification systems (GE Healthcare) or glass econo-columns (Bio-Rad) at room temperature.

\section{Isothermal titration calorimetry (ITC) of peptides and ligands}

Titrations were performed on an ITC200 micro-calorimeter (GE Healthcare). The peptides were dissolved in buffer (20 mM Bis-Tris propane, $100 \mathrm{mM} \mathrm{NaCl}, 1 \mathrm{mM}$ tris(2-carboxyethyl)phosphine 
(TCEP), $\mathrm{pH}$ 7.4) at $100 \mu \mathrm{M}$. The compounds were titrated against $10 \mu \mathrm{M}$ VBC complex, equilibrated in the same buffer. The titrations consisted of 20 injections of $2 \mu \mathrm{L}$ of peptide solution at a rate of 0.5 $\mu \mathrm{L} / \mathrm{s}$ at $120 \mathrm{~s}$ time intervals. An initial injection of ligand $(0.4 \mu \mathrm{L})$ was made and discarded during data analysis. All experiments were performed at $25^{\circ} \mathrm{C}$ while stirring at $650 \mathrm{rpm}$.

Ligands 14a-b were diluted from DMSO stock solution to $300 \mu \mathrm{M}$ in buffer (20 mM Bis-Tris propane, $150 \mathrm{mM} \mathrm{NaCl}, 1 \mathrm{mM}$ TCEP, $\mathrm{pH}$ 7.4). The compounds were titrated against $30 \mu \mathrm{M} \mathrm{VBC}$ complex, equilibrated in the same buffer. The final concentration of DMSO in each experiment was $3 \%(\mathrm{v} / \mathrm{v})$.

The data were fitted to a single-binding-site model using the MicroCal PEAQ-ITC analysis software provided by the manufacturer to obtain the stoichiometry $n$, the dissociation constant $K_{\mathrm{d}}$, and the enthalpy of binding $\Delta H$. The reported values are the mean \pm s.e.m. from independent measurements.

\section{Isothermal titration calorimetry (ITC) of PROTACs}

The titrations were all performed as reverse mode (protein in syringe, ligand in cell) and consisted of 19 injections of $2 \mu \mathrm{l}$ protein solution $(20 \mathrm{mM}$ Bis-Tris propane, $100 \mathrm{mM} \mathrm{NaCl}, 1 \mathrm{mM}$ tris(2carboxyethyl)phosphine (TCEP), $\mathrm{pH} 7.4$ ) at a rate of $0.5 \mu \mathrm{l} / \mathrm{s}$ at $120 \mathrm{~s}$ time intervals. An initial injection $(0.4 \mu \mathrm{l})$ was made and discarded during data analysis. All experiments were performed at 25 ${ }^{\circ} \mathrm{C}$, whilst stirring at 600 r.p.m.

PROTACs (15a or 15b) were diluted from a $10 \mathrm{mM}$ DMSO stock solution to $20 \mu \mathrm{M}$ in buffer containing $20 \mathrm{mM}$ Bis-Tris propane, $100 \mathrm{mM} \mathrm{NaCl}, 1 \mathrm{mM}$ tris(2-carboxyethyl)phosphine (TCEP), $\mathrm{pH}$ 7.4. The final DMSO concentration was $0.2 \%$. Bromodomain (100 or $200 \mu \mathrm{M}$, in the syringe) was titrated into the PROTAC $(20 \mu \mathrm{M}$, in the cell). At the end of the titration, the excess of solution was removed from the cell, the syringe was washed and dried, VCB complex (168 $\mu \mathrm{M}$, in the same buffer) was loaded in the syringe and titrated into the complex PROTAC-bromodomain. The concentration of the complex in the cell $(C)$ after the first titration $(16.8 \mu \mathrm{M})$, was calculated as follows:

$C=\frac{C_{0} \cdot V_{\text {cell }}}{V_{\text {cell }}+V_{\text {inj }}}$

where: $C_{0}$ is the initial concentration of the PROTAC in the cell $(20 \mu \mathrm{M}), V_{\text {cell }}$ is the volume of the sample cell $(200.12 \mu \mathrm{l})$ and $V_{\text {inj }}$ is the volume of titrant injected during the first titration $(38.4 \mu \mathrm{l})$. Titrations for the binary complex PROTAC-VCB were performed as follows: to the solution of PROTAC $(20 \mu \mathrm{M}$, in the cell), buffer $(38.4 \mu \mathrm{L})$ was added by means of a single ITC injection. The excess solution was removed from the cell, the syringe was washed and dried and VCB complex (168 $\mu \mathrm{M}$, in the same buffer) was loaded in the syringe and titrated into the diluted PROTAC solution. The data were fitted to a single-binding-site model to obtain the stoichiometry $n$, the dissociation constant $K_{\mathrm{d}}$ and the enthalpy of binding $\Delta H$ using the d analyzed using the MicroCal PEAQ-ITC 
analysis software provided by the manufacturer. The reported values are the mean \pm s.e.m. from independent measurements.

\section{X-Ray crystallography of protein complexes}

To obtain the peptide 13a:VBC cocrystal structure, purified VBC complex was incubated for $30 \mathrm{~min}$ with a 4 -fold excess of peptide 13a, then loaded in a Superdex 75 10/300 column (GE Healthcare) to remove excess peptide. Liquor solutions containing $0.1 \mathrm{M}$ potassium phosphate, $\mathrm{pH}=6.0-6.2,20-$ $22 \%(\mathrm{w} / \mathrm{v})$ polyethylene glycol monomethyl ether 5000, 0.2 M ammonium sulphate, and $10 \mathrm{mM}$ DTT were prepared based on published work using a hydroxylated HIF-1 $\alpha$ peptide (PDB: 1lqb, ref. ${ }^{17}$ ). Crystals were obtained by mixing equal volumes of liquor solution and peptide 13a:VBC complex $(\sim 5 \mathrm{mg} / \mathrm{mL})$ using a hanging-drop diffusion method at $18^{\circ} \mathrm{C}$.

For the small molecule soaked VBC structures, equal volume solutions of VBC $(\sim 5 \mathrm{mg} / \mathrm{mL})$ and liquor solution were mixed in the hanging-drop vapor diffusion method at $18^{\circ} \mathrm{C}$. The liquor solution contained $0.1 \mathrm{mM}$ sodium cacodylate, $\mathrm{pH} 6.2-6.6,16-18 \%$ polyethylene glycol $3350,0.2 \mathrm{M}$ magnesium acetate and $10 \mathrm{mM}$ DTT. The drop was streaked with seeds of disrupted VBC crystals and a 2-3 mm layer of Al's Oil (Hampton Research) was applied on top of the liquor solution to slow the vapour diffusion rate. To obtain the structure of ligand 14a bound to VBC, the crystal was soaked overnight in a $2 \mathrm{mM}$ solution of $\mathbf{1 4 a}$ in $10 \% \mathrm{DMSO}, 40 \%$ isopropanol and $50 \%$ liquor solution. To obtain the structure of ligand $\mathbf{1 4 b}$ bound to VBC, the crystal was soaked overnight in a $1 \mathrm{mM}$ solution of $14 \mathrm{~b}$ in $1 \%$ DMSO, $4 \%$ isopropanol and $95 \%$ liquor solution.

Crystals were screened using an in-house Rigaku M007HF x-ray generator and Saturn 944HG+ CCD detector. X-ray data were collected at $100 \mathrm{~K}$ at Diamond Light Source beamlines I04 and I04-1. Indexing and integration of reflections was performed using XDS with the XDSGUI interface ${ }^{18}$ and scaling and merging with AIMLESS ${ }^{19}$ in CCP4i $\left(\right.$ ref. $\left.^{20}\right)$. The isomorphous datasets were refined using REFMAC5 (ref. ${ }^{21}$ ) and COOT $^{22}$ using template structures derived from the Protein Data Bank (PDB) entries $1 \mathrm{vcb}^{23}$ and 1lqb (ref. ${ }^{17}$ ). Ligand structures and restraints were generated using the PRODRG server $^{24}$. The MOLPROBITY server was used to validate the geometry and steric clashes in the structures $^{25}$. The structures have been deposited in the PDB with accession codes 6GFX, 6GFY 6GFZ and data collection and refinement statistics are presented in Supplementary Table 6. 


\section{Tissue culture}

HeLa and A549 cells were kept in DMEM (Gibco) supplemented with 10\%(v/v) FBS (Gibco), 2 mM L-glutamine (Gibco), penicillin (100 units $/ \mathrm{mL}$ ) and streptomycin $(100 \mu \mathrm{g} / \mathrm{mL})$ (Gibco). MV4;11 cells were kept in RPMI 1640 supplemented with 10\%(v/v) FBS (Gibco), 2 mM L-glutamine (Gibco), penicillin (100 units $/ \mathrm{mL})$ and streptomycin $(100 \mu \mathrm{g} / \mathrm{mL})$ (Gibco). Cells were kept at $37{ }^{\circ} \mathrm{C}, 5 \% \mathrm{CO}_{2}$.

\section{Testing compounds in cells}

HeLa cells $\left(3 \times 10^{5}\right)$ were seeded onto each well on a six-well plate $24 \mathrm{~h}$ before treatment with test compounds. To treat the cells with the test compounds, medium was replaced with fresh medium containing specified concentration of test compounds for specified time. At the end of treatment, cells were washed with PBS and then were lysed by applying RIPA buffer (Sigma, R0278) supplemented with Protease inhibitor (Roche, \# 04693159001) and Benzonase (Sigma, E1014). Lysate was clarified by centrifugation $\left(20,000 \times \mathrm{g}, 10 \mathrm{~min}, 4{ }^{\circ} \mathrm{C}\right)$. Supernatant was collected and protein concentration of was determined by BCA assay. The rest of the clarified lysate was kept at $-80^{\circ} \mathrm{C}$ before further processing.

\section{Immunoblotting}

Protein on gel was transferred to nitrocellulose membrane using Bio-Rad Transblot system according to manufacturer guidelines. Blots were probed with anti-Brd4 (AbCam, ab128874), anti-Brd3 (AbCam, ab50818), anti-Brd2 (AbCam, ab139690) and anti- $\beta$-actin (Cell Signaling, no. 4970) antibodies. Blots were developed with secondary anti-mouse IgG (Licor, 926-32210) or anti-rabbit IgG (Licor, 926-32213) antibodies from Licor and bands visualized using Bio-Rad ChemiDoc MP Imaging System.

\section{Western Blot Quantification}

Image processing and band intensity quantification were performed using Bio-Rad Image Lab software version 6.0.0. Reported band intensities are normalized to loading control, i.e., $\beta$-actin or tubulin for total lysates. 


\section{Cell Viability Assay}

MV4;11 cells ( 15000 cells per well) were dosed for $72 \mathrm{~h}$ with compounds serially diluted (1:3) on a clear-bottom 384-well plate with a final concentration of $0.05 \%(\mathrm{v} / \mathrm{v})$ DMSO. After treatment, Promega CellTiter-Glo luminescent cell viability assay (G7572) was added to the cells according to the manufacturer instructions. The signal from each well was recorded on a BMG Labtech Pherastar luminescence plate reader with recommended settings.

Normalized data were analyzed and plotted with Graphpad Prism software in the ' $\log$ (inhibitor) vs. response -- Variable slope (four parameters)' module. $\mathrm{EC}_{50}$ values of each test compound were derived from this plot.

$\underline{\mathrm{EC}_{50}} \underline{\text { Table }}$

\begin{tabular}{|l|l|l|l|l|}
\hline & MZ1 & $15 \mathrm{~b}$ & $15 \mathrm{a}$ & Cis-MZ1 \\
\hline $\mathrm{EC}_{50}$ & $\mathbf{5 . 4 7 E - 0 8}$ & $\sim \mathbf{0 . 0 2 4 6 4}$ & $\mathbf{3 . 3 0 E - 0 7}$ & $\sim \mathbf{0 . 0 0 0 2 2 7 2}$ \\
\hline $\mathrm{pEC}_{50}$ & $\mathbf{7 . 2 6 2}$ & $\sim \mathbf{1 . 6 0 8}$ & $\mathbf{6 . 4 8 2}$ & $\sim \mathbf{3 . 6 4 4}$ \\
\hline
\end{tabular}




\section{A549 Colony-Formation Assay}

A549 cells were seeded in clear, Nunc ${ }^{\mathrm{TM}}$ Edge 2.0 96-well plates (Thermo Scientific) at 1000 cells per well and incubated at $37^{\circ} \mathrm{C}$ in DMEM. After $24 \mathrm{~h}$ cells were treated with test compounds from $10 \mu \mathrm{M}$ to $100 \mathrm{nM}$, with a final DMSO of $0.1 \%$. The cells were left to grow until DMSO-treated wells reached $\sim 100 \%$ confluence ( $\sim 8$ days), at which point they were washed with PBS, fixed with $100 \% \mathrm{MeOH}$ (30 $\min 4^{\circ} \mathrm{C}$ ), stained with $0.1 \%$ crystal violet $(30 \mathrm{~min}$ at r.t.) and left to dry overnight. Once dried plates were imaged on an Epson Perfection V800 scanner, at a resolution of 1200 DPI and in 48-bit colour.

For dose-response curves, crystal-violet dye was extracted by the addition of $200 \mu 11 \%$ SDS to each well, and incubation for $3 \mathrm{~h}$ at r.t. The absorbance at $595 \mathrm{~nm}$ of each well was measured on a Pherastar FS (BMG), normalised for each compound and plotted against compound concentration.

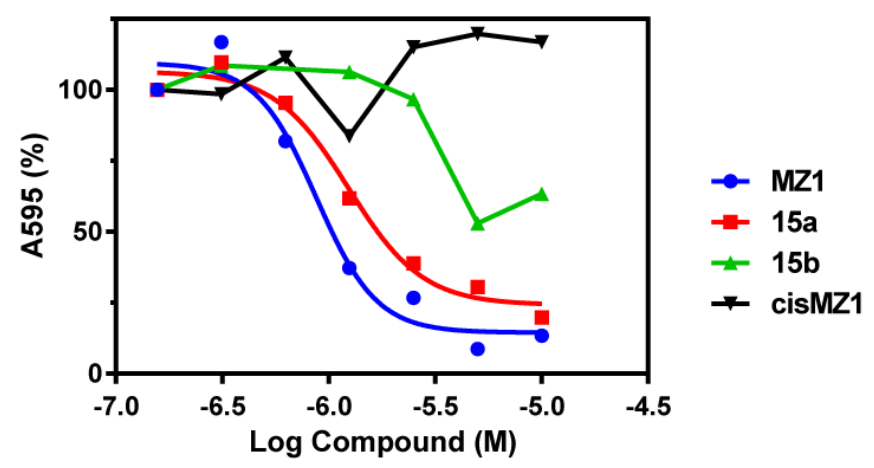

\begin{tabular}{|c|cc|}
\hline $\mathrm{A} 549$ & $\mathrm{MZ1}$ & $15 \mathrm{a}$ \\
\hline $\mathrm{IC}_{50}(\mathrm{nM})$ & 875 & 1239 \\
$\mathrm{Imax}(\%)$ & 86 & 76 \\
$\mathrm{pIC}_{50}$ & 6.1 & 5.9 \\
$\mathrm{SE}$ & 0.08 & 0.06 \\
$\mathrm{R}^{2}$ & 0.97 & 0.98 \\
\hline
\end{tabular}




\section{Plasma stability}

Test compound $(10 \mu \mathrm{M})$ was incubated in pre-warmed plasma at $37^{\circ} \mathrm{C}$ (that is buffered to $\mathrm{pH} 7.4$ in ratio of 70:30 plasma to buffer). Immediately, at time zero, then at 30,60, 120, and $180 \mathrm{~min}$, a $50 \mu \mathrm{L}$ aliquot of the incubation mixture was removed and mixed with $200 \mu \mathrm{L}$ acetonitrile containing Donepezil as the internal standard $(50 \mathrm{ng} / \mathrm{ml})$ to stop the reaction. The samples were centrifuged to sediment the precipitated protein and the plates then sealed prior to UPLC-MS/MS analysis using a Quattro Premier XE (Waters Corporation, USA).

XLfit (IDBS, UK) was used to calculate the exponential decay and consequently the rate constant (k) from the ratio of peak area of test compound to internal standard at each time point. The half-life was calculated for each test compound from the rate by using the following calculation:

$$
\mathrm{t}_{1 / 2}=0.693 / \mathrm{k}
$$

\section{Intrinsic clearance}

Test compound $(0.5 \mu \mathrm{M})$ was incubated with female CD1 mouse liver microsomes (Xenotech LLC TM; $0.5 \mathrm{mg} / \mathrm{mL}$ in $50 \mathrm{mM}$ potassium phosphate buffer, $\mathrm{pH}$ 7.4) and the reaction started with addition of excess NADPH ( $8 \mathrm{mg} / \mathrm{mL} 50 \mathrm{mM}$ potassium phosphate buffer, $\mathrm{pH}$ 7.4). Immediately, at time zero, then at $3,6,9,15$ and $30 \mathrm{~min}$ an aliquot $(50 \mu \mathrm{L})$ of the incubation mixture was removed and mixed with acetonitrile $(100 \mu \mathrm{L})$ to stop the reaction. Internal standard was added to all samples, the samples centrifuged to sediment precipitated protein and the plates then sealed prior to UPLC-MS/MS analysis using a Quattro Premier XE (Waters Corporation, USA).

XLfit (IDBS, UK) was used to calculate the exponential decay and consequently the rate constant (k) from the ratio of peak area of test compound to internal standard at each time point. The rate of intrinsic clearance (CLi) of each test compound was then calculated using the following calculation:

\section{$\mathrm{CLi}(\mathrm{mL} / \mathrm{min} / \mathrm{g}$ liver $)=\mathrm{k}$ x V x Microsomal protein yield}

Where $\mathrm{V}$ ( $\mathrm{mL} / \mathrm{mg}$ protein) is the incubation volume $/ \mathrm{mg}$ protein added and microsomal protein yield is taken as $52.5 \mathrm{mg}$ protein/ $\mathrm{g}$ liver. Verapamil $(0.5 \mu \mathrm{M})$ was used as a positive control to confirm acceptable assay performance. 


\section{Parallel Artificial Membrane Permeability Assay (PAMPA)}

PAMPA was performed using a 96-well pre-coated BD Gentest ${ }^{\mathrm{TM}}$ PAMPA plate (BD Biosciences, U.K.). Each well was divided into two chambers; donor and acceptor, separated by a lipid-oil-lipid trilayer constructed in a porous filter. The effective permeability, $\mathrm{P}_{\mathrm{e}}$, of the compound was measured at $\mathrm{pH}$ 7.4. Stock solutions $(5 \mathrm{mM})$ of the compound were prepared in DMSO. The compound was then further diluted to $10 \mu \mathrm{M}$ in phosphate buffered saline at $\mathrm{pH}$ 7.4. The final DMSO concentration did not exceed $5 \% \mathrm{v} / \mathrm{v}$. The compound dissolved in phosphate buffered saline was then added to the donor side of the membrane and phosphate buffered saline without compound was added to the acceptor side. The PAMPA plate was left at room temperature for $5 \mathrm{~h}$. After which time, an aliquot (100 $\mu \mathrm{l})$ was then removed from both acceptor and donor compartments and mixed with acetonitrile $(80 \mu \mathrm{l})$ containing an internal standard. The samples were centrifuged $\left(10 \mathrm{~min}, 5{ }^{\circ} \mathrm{C}, 3270 \mathrm{~g}\right)$ to sediment precipitated protein and sealed prior to UPLC-MS/MS analysis using a Quattro Premier XE (Waters Corp, USA). $\mathrm{P}_{\mathrm{e}}$ was calculated as shown in the below equation:

$$
\mathrm{P}_{\mathrm{e}}\left(\mathrm{nm} \mathrm{s}^{-1}\right)=\frac{10^{7} \times-\ln \left[1-C_{A}(t) / C_{\text {equi }}\right]}{\mathrm{A} \times\left(\frac{1}{V_{D}}+\frac{1}{V_{A}}\right) \times \mathrm{t}}
$$

Where:

$C_{A}(t)=$ peak area of compound present in acceptor well at time $\mathrm{t}=18000 \mathrm{~s}$

$C_{\text {equi }}=\left[\mathrm{C}_{\mathrm{D}}(\mathrm{t}) \times \mathrm{V}_{\mathrm{D}}+\mathrm{C}_{\mathrm{A}(\mathrm{t})} \times \mathrm{V}_{\mathrm{A}}\right] /\left(\mathrm{V}_{\mathrm{D}}+\mathrm{V}_{\mathrm{A}}\right)$

$\mathrm{A} \quad=$ filter area

$V_{D} \quad=$ donor well volume

$V_{A} \quad=$ acceptor well volume

$t \quad=$ incubation time

$C_{D}(t)=$ peak area of compound present in donor well at time $\mathrm{t}=18000 \mathrm{~s}$

Recovery of compound from donor and acceptor wells was calculated and data was only accepted when recovery exceeded $70 \%$. 


\section{CHI Log D determination}

Using a fast gradient, reverse-phase HPLC method to determine the chromatographic hydrophobicity index (CHI) was determined as described by Camurri and Zaramella (ref. ${ }^{26}$ ) and Valko et al. (ref. ${ }^{27}$ ). Briefly, by plotting the retention time of a set of reference compounds against known CHI values. The $\mathrm{CHI}$ value of test compounds was then calculated according to their retention time.

Test compounds were prepared as $0.5 \mathrm{mM}$ solutions in 50:50 acetonitrile:water and analysed by reversed-phase HPLC-UV (wavelength $254 \mathrm{~nm}$ ) using a Phenomenex Luna C18 $100 \AA 150$ x 4.6mm 5 micron column with a gradient of aqueous phase (50 mM ammonium acetate, $\mathrm{pH} 7.4)$ and mobile phase (acetonitrile). 


\section{ITC titrations}

$13 a$ against VBC
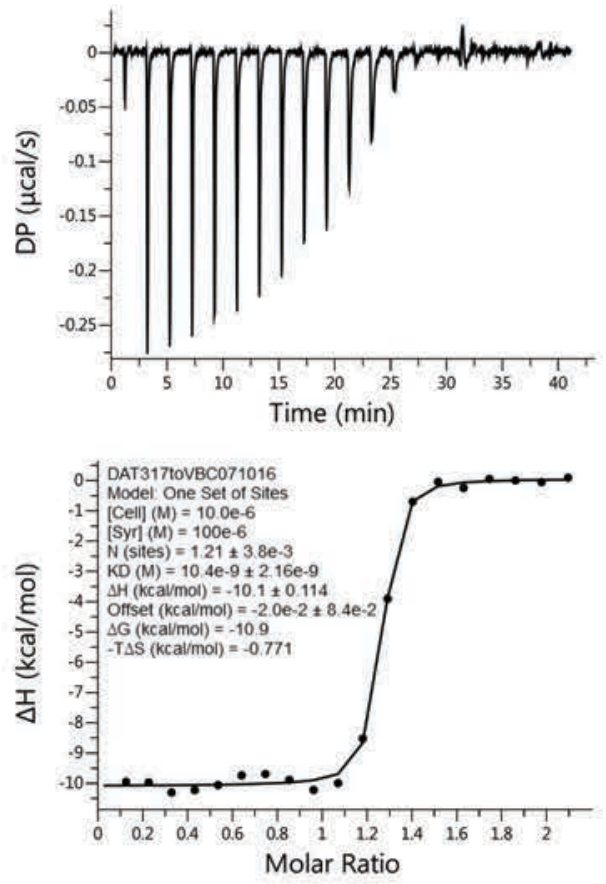

$13 c$ against VBC
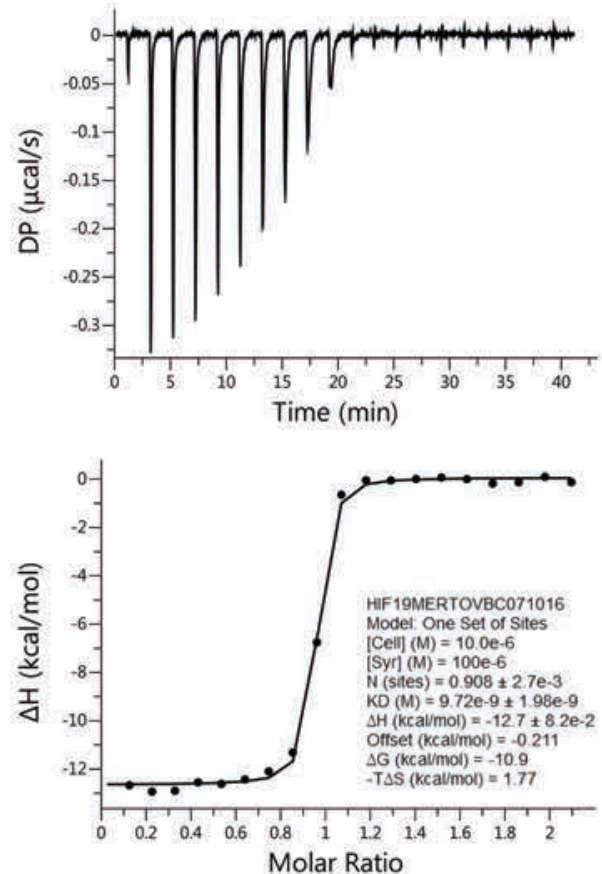

$13 b$ against VBC
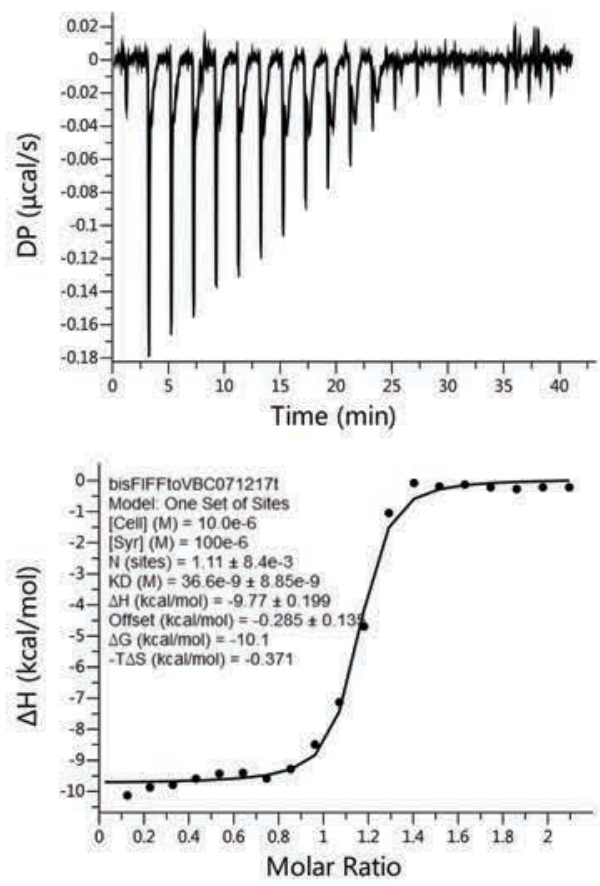

14a against VBC
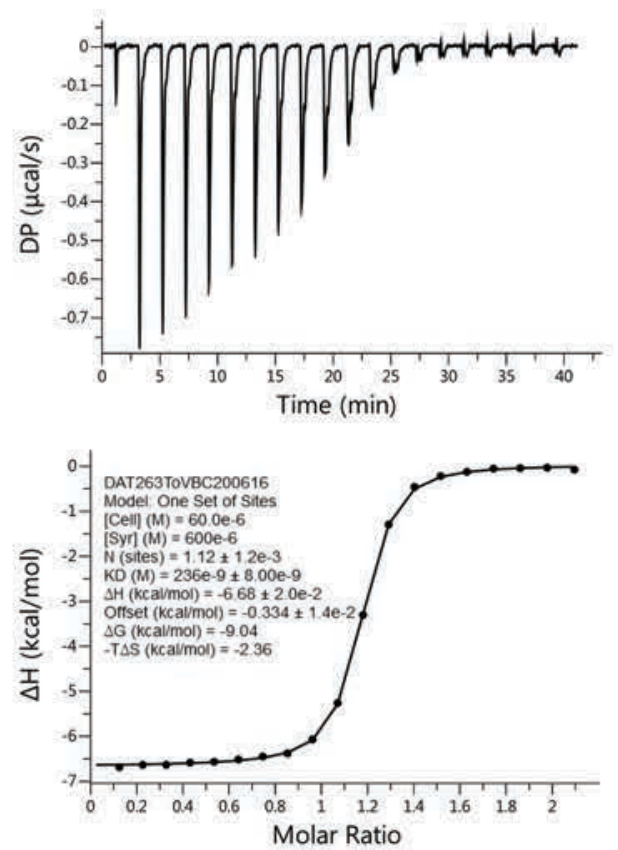
$14 \mathrm{~b}$ against VBC
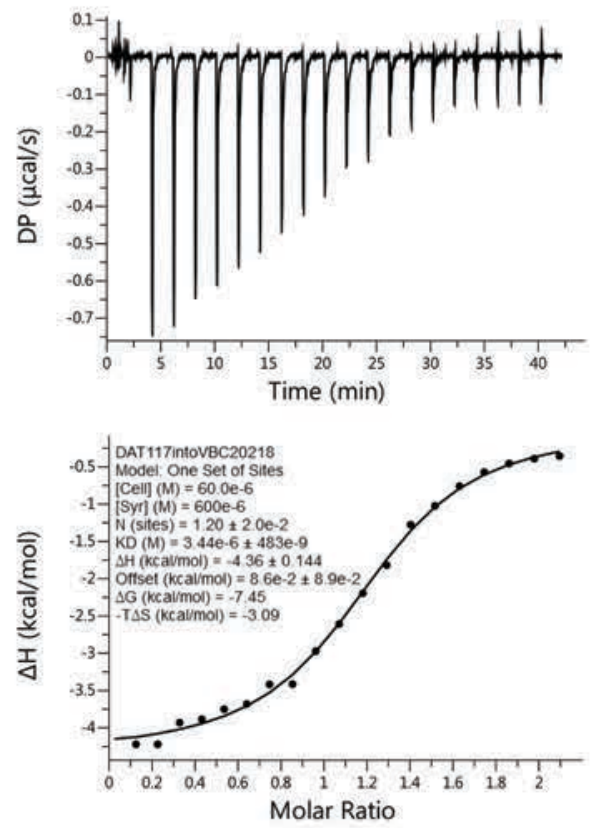

VBC against $15 b$
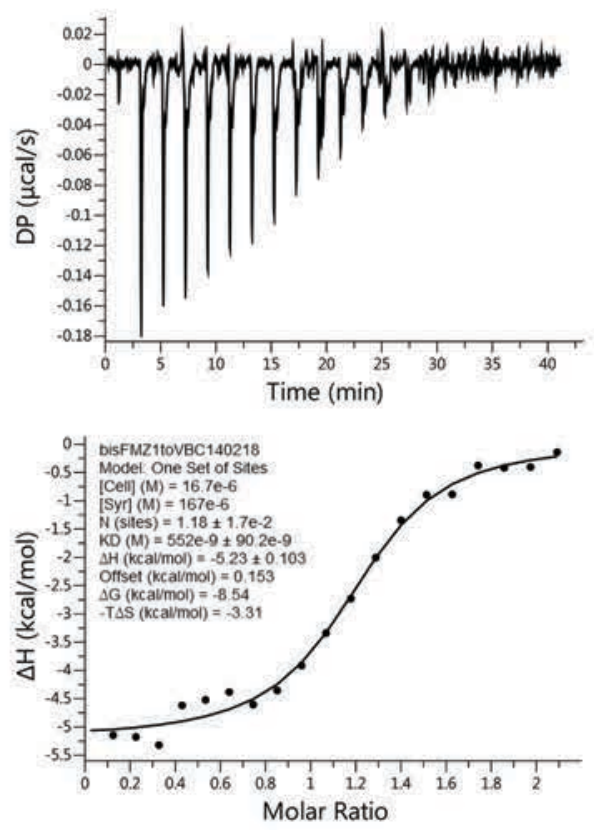

VBC against $15 a$
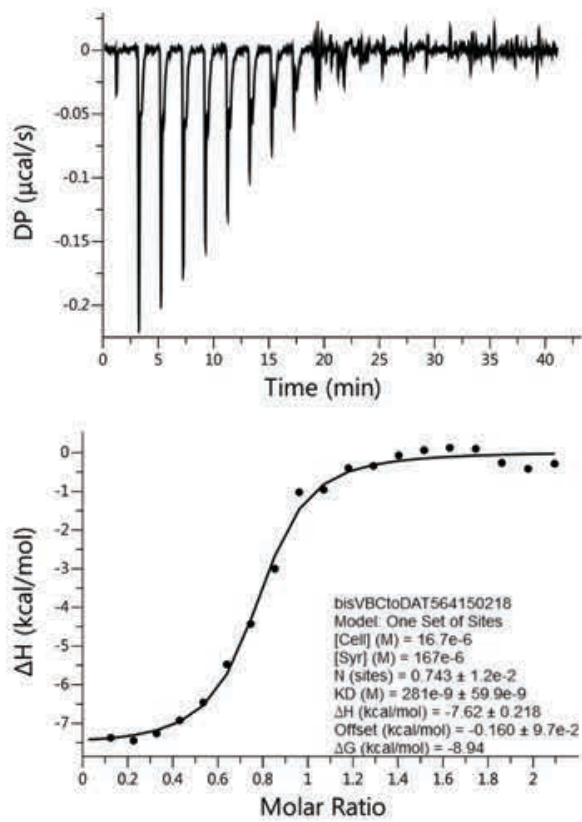

Brd2BD1 against 15a
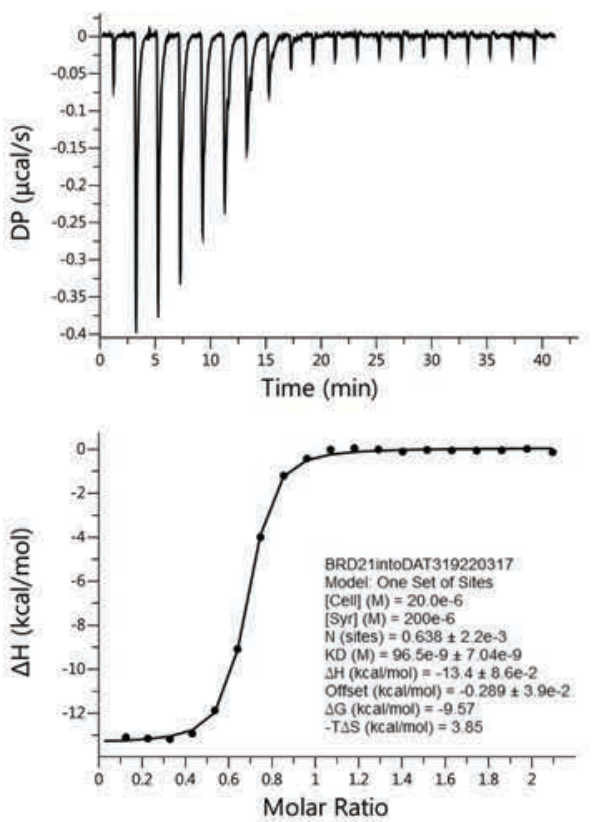
Brd4BD2 against 15a
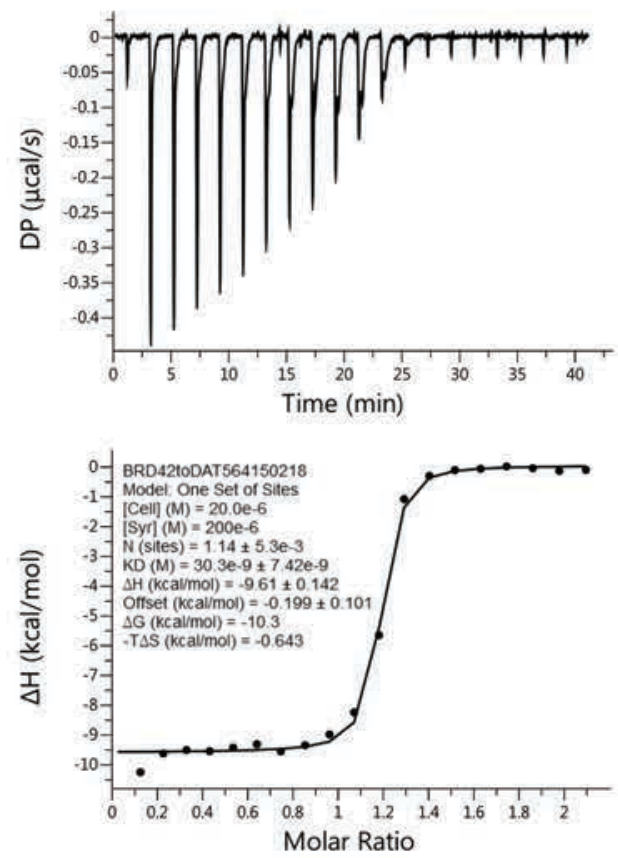

VBC against Brd4BD2:15a
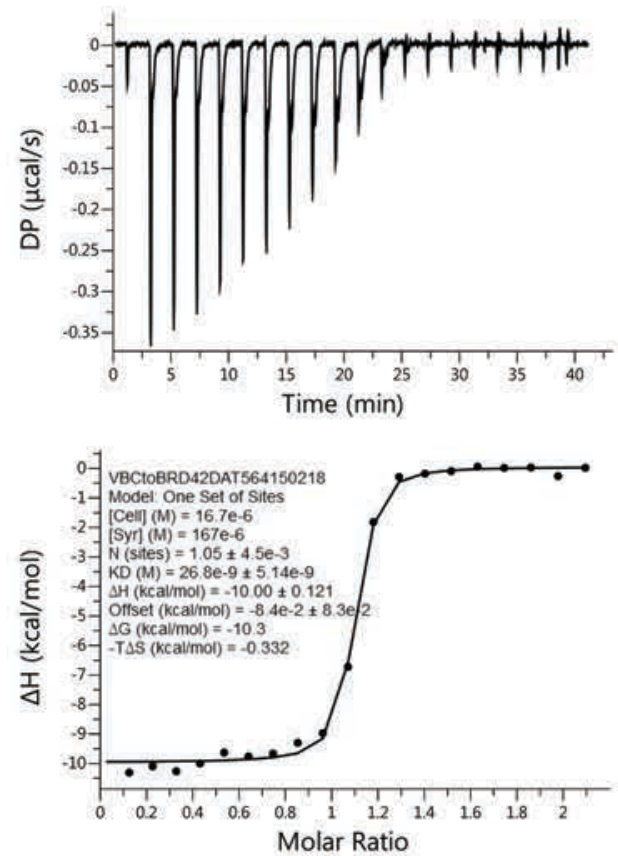

VBC against Brd2BD1:15a
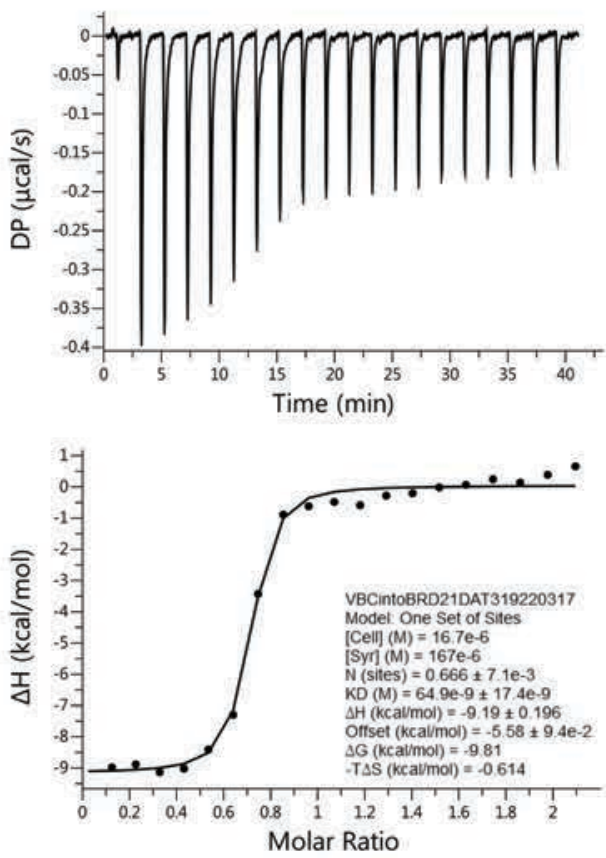

VBC against $B r d 2 B D 1: 15 b$
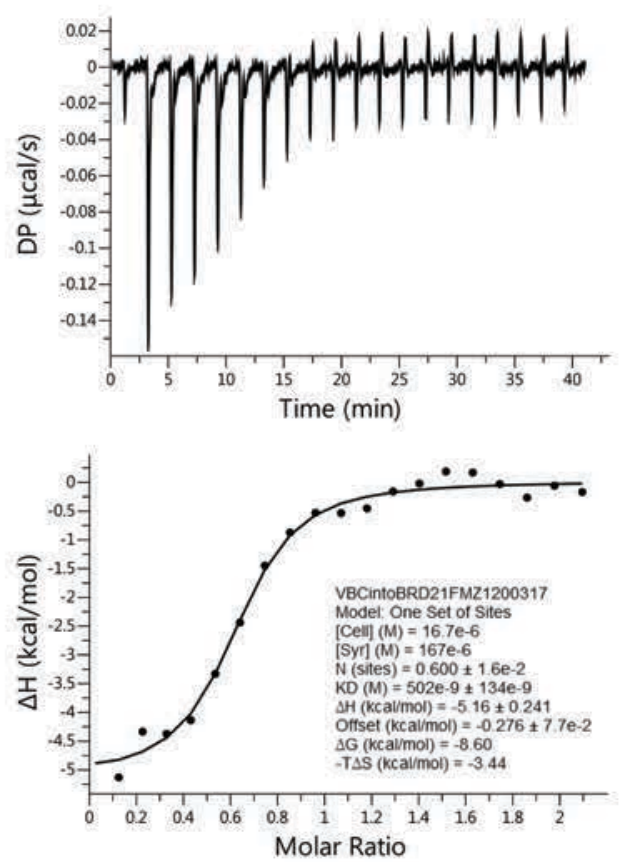
VBC against Brd4BD2:15b
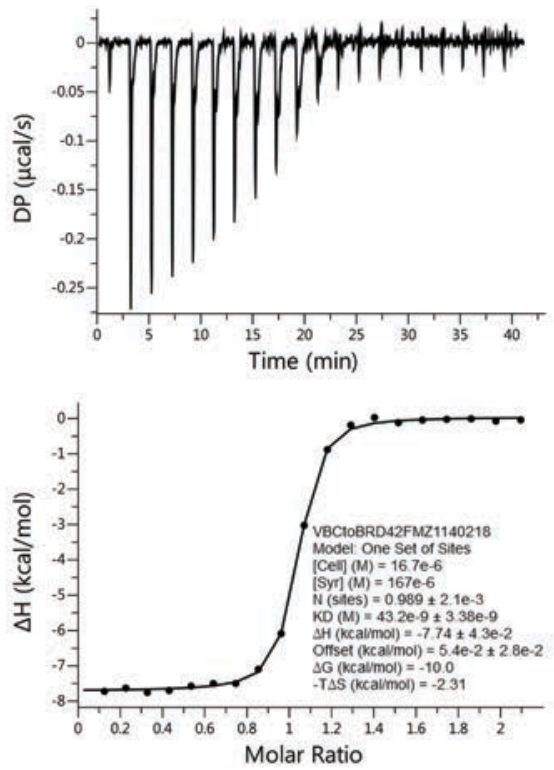


\section{References}

(1) Bretscher, L. E.; Jenkins, C. L.; Taylor, K. M.; DeRider, M. L.; Raines, R. T. J Am Chem Soc 2001, 123, 777.

(2) Kim, W.; Hardcastle, K. I.; Conticello, V. P. Angewandte Chemie International Edition $2006,45,8141$.

(3) Graton, J.; Wang, Z.; Brossard, A. M.; Monteiro, D. G.; Le Questel, J. Y.; Linclau, B. Angewandte Chemie-International Edition 2012, 51, 6176.

(4) Cortis, C. M.; Langlois, J. M.; Beachy, M. D.; Friesner, R. A. Journal of Chemical Physics 1996, 105, 5472.

(5) Glendening, E. D.; Landis, C. R.; Weinhold, F. J Comput Chem 2013, 34, 1429.

(6) Johnson, E. R.; Keinan, S.; Mori-Sánchez, P.; Contreras-García, J.; Cohen, A. J.; Yang, W. Journal of the American Chemical Society 2010, 132, 6498.

(7) Bader, R. F. W.; Essén, H. The Journal of Chemical Physics 1984, 80, 1943.

(8) Lu, T.; Chen, F. W. J Comput Chem 2012, 33, 580.

(9) Kenny, P. W. Journal of Chemical Information and Modeling 2009, 49, 1234.

(10) Galdeano, C.; Gadd, M. S.; Soares, P.; Scaffidi, S.; Van Molle, I.; Birced, I.; Hewitt, S.;

Dias, D. M.; Ciulli, A. Journal of Medicinal Chemistry 2014, 57, 8657.

(11) Olsson, M. H. M.; Søndergaard, C. R.; Rostkowski, M.; Jensen, J. H. Journal of Chemical Theory and Computation 2011, 7, 525.

(12) Søndergaard, C. R.; Olsson, M. H. M.; Rostkowski, M.; Jensen, J. H. Journal of Chemical Theory and Computation 2011, 7, 2284.

(13) Sheldrick, G. Acta Crystallographica Section C 2015, 71, 3.

(14) Spek, A. Acta Crystallographica Section C 2015, 71, 9.

(15) Farrugia, L. Journal of Applied Crystallography 1997, 30, 565.

(16) Flack, H. Acta Crystallographica Section A 1983, 39, 876.

(17) Hon, W. C.; Wilson, M. I.; Harlos, K.; Claridge, T. D.; Schofield, C. J.; Pugh, C. W.; Maxwell, P. H.; Ratcliffe, P. J.; Stuart, D. I.; Jones, E. Y. Nature 2002, 417, 975.

(18) Kabsch, W. Acta Crystallographica Section D 2010, 66, 133.

(19) Evans, P. R.; Murshudov, G. N. Acta Crystallographica Section D 2013, 69, 1204.

(20) Winn, M. D.; Ballard, C. C.; Cowtan, K. D.; Dodson, E. J.; Emsley, P.; Evans, P. R.; Keegan, R. M.; Krissinel, E. B.; Leslie, A. G. W.; McCoy, A.; McNicholas, S. J.; Murshudov, G. N.; Pannu, N. S.; Potterton, E. A.; Powell, H. R.; Read, R. J.; Vagin, A.; Wilson, K. S. Acta Crystallographica Section D 2011, 67, 235.

(21) Murshudov, G. N.; Skubak, P.; Lebedev, A. A.; Pannu, N. S.; Steiner, R. A.; Nicholls, R. A.; Winn, M. D.; Long, F.; Vagin, A. A. Acta Crystallographica Section D 2011, 67, 355.

(22) Emsley, P.; Lohkamp, B.; Scott, W. G.; Cowtan, K. Acta Crystallographica Section D 2010, 66, 486.

(23) Stebbins, C. E.; Kaelin, W. G., Jr.; Pavletich, N. P. Science 1999, 284, 455.

(24) Schuttelkopf, A. W.; van Aalten, D. M. F. Acta Crystallographica Section D 2004, 60, 1355.

(25) Chen, V. B.; Arendall, W. B., III; Headd, J. J.; Keedy, D. A.; Immormino, R. M.; Kapral, G. J.; Murray, L. W.; Richardson, J. S.; Richardson, D. C. Acta Crystallographica Section D 2010, 66, 12.

(26) Camurri, G.; Zaramella, A. Anal Chem 2001, 73, 3716.

(27) Valko, K.; Nunhuck, S.; Bevan, C.; Abraham, M. H.; Reyncilds, D. P. J Pharm Sci-Us 2003, 92, 2236. 
NMR Spectra 
${ }^{1} \mathrm{H}-\mathrm{NMR}, \mathrm{CDCl}_{3}$

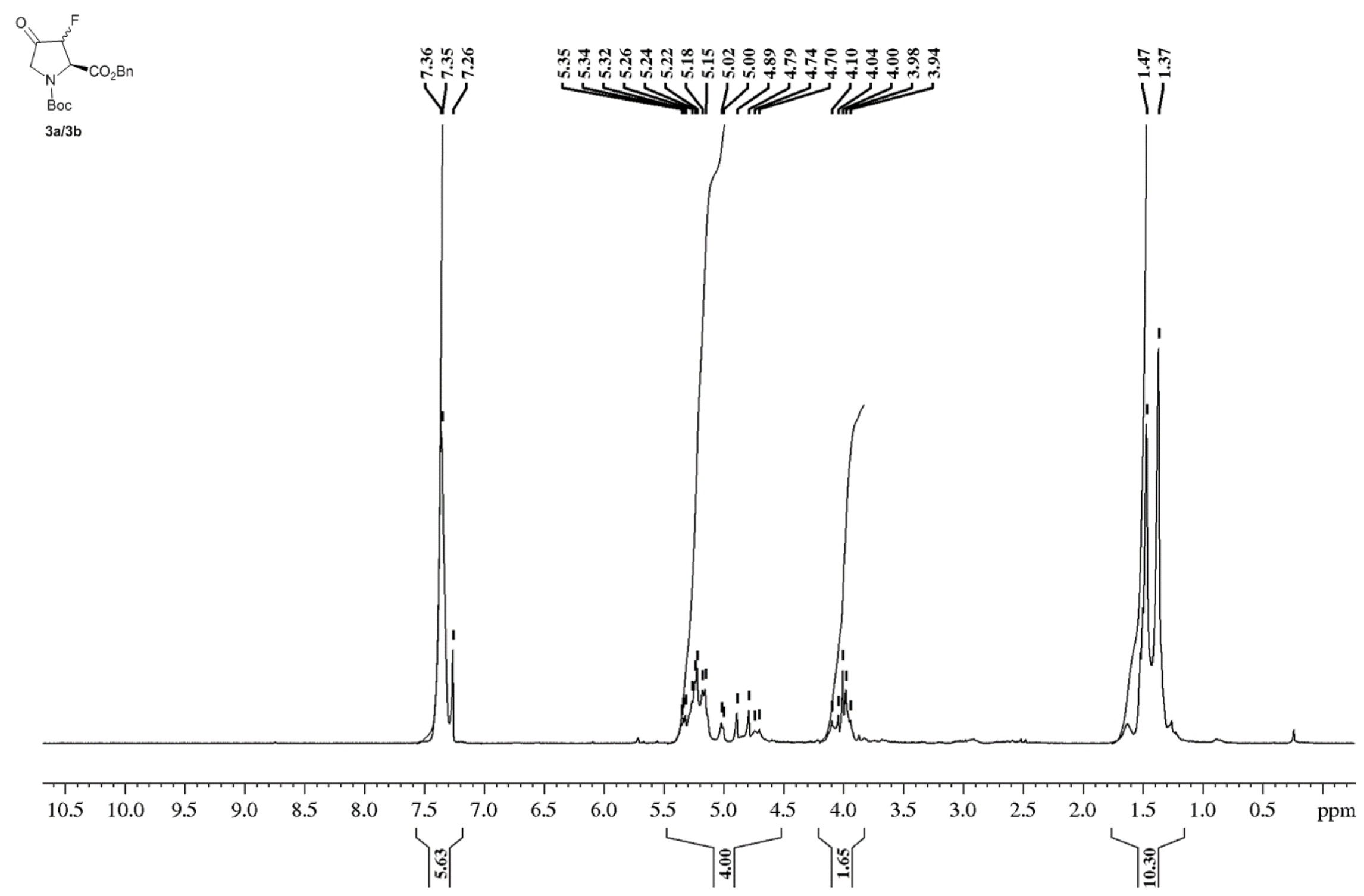


${ }^{19} \mathrm{~F}-\mathrm{NMR}, \mathrm{CDCl}_{3}$

F19CPD.d CDC13 \{C: \Bruker \TopSpin3.2\} ALC 22

\section{arigere \\ ( $)$}

$\sum_{\substack{\mathrm{B} \\ \mathrm{Boc} \\ 3 \mathrm{a} / 3 \mathrm{~b}}}^{\mathrm{F}} \mathrm{CO}_{2} \mathrm{Bn}$
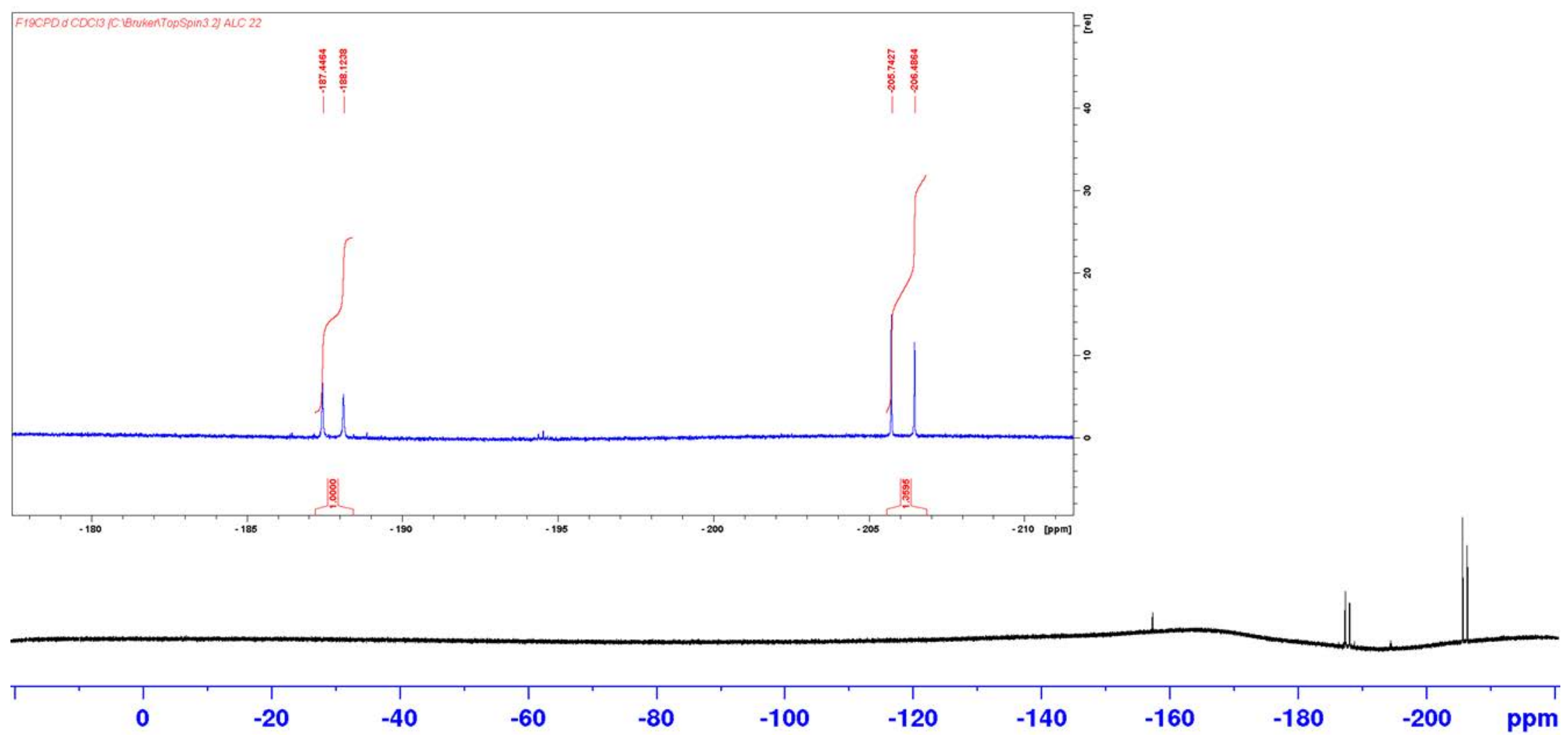
${ }^{1} \mathrm{H}-\mathrm{NMR}, \mathrm{CDCl}_{3}$

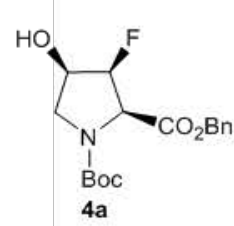

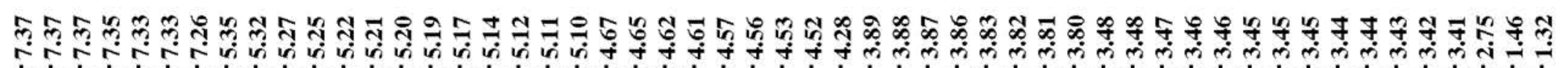

Boc
$4 a$
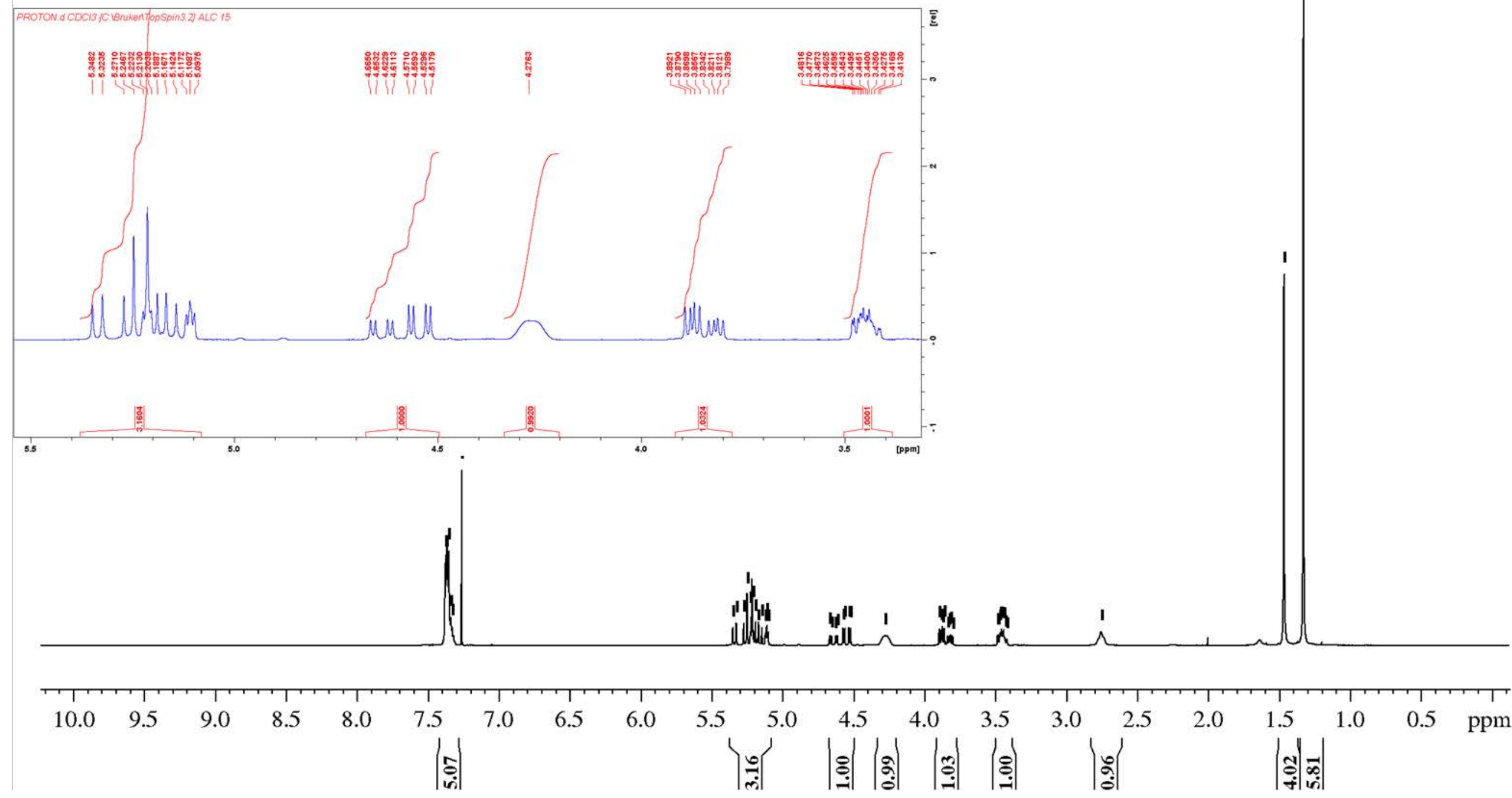
${ }^{19} \mathrm{~F}-\mathrm{NMR}, \mathrm{CDCl}_{3}$

\section{expigrex \\ $\mathrm{C} \times$}

$\sum_{\substack{N \\ \text { Boc } \\ 4 a}}^{\mathrm{CO}}$

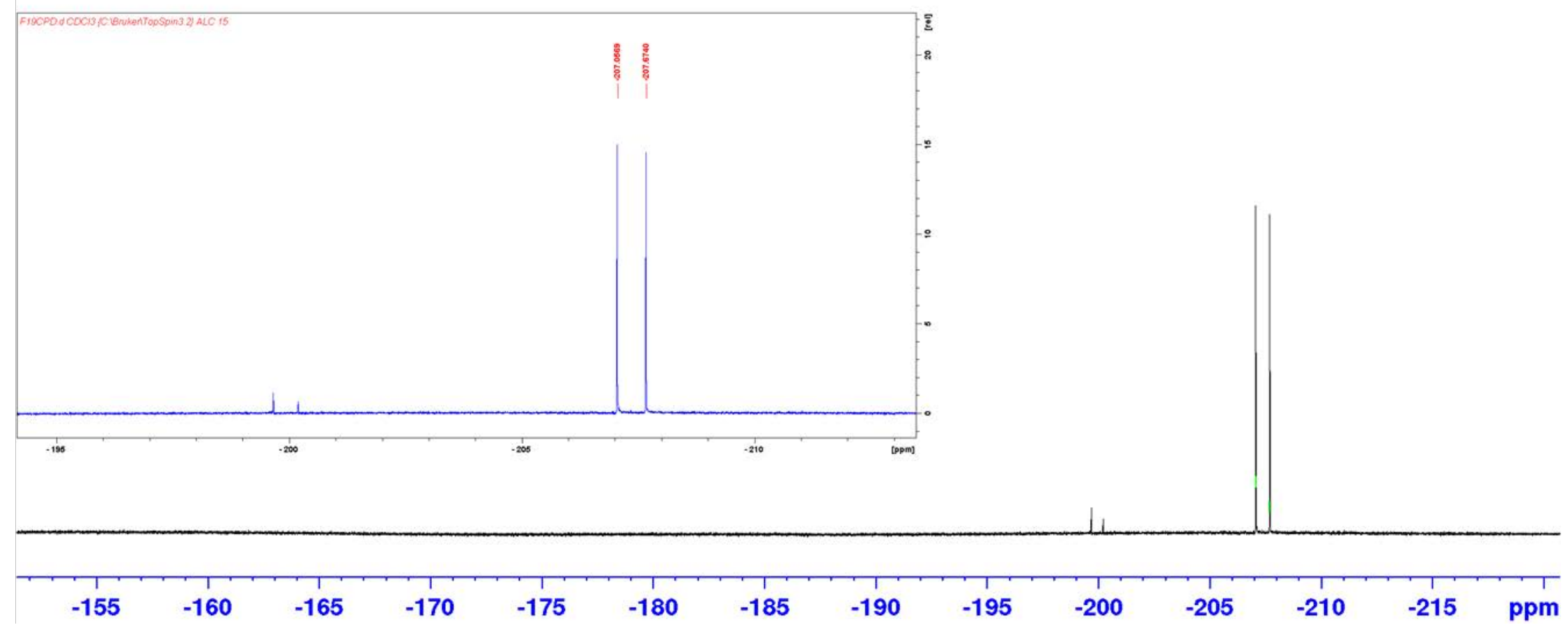


${ }^{13} \mathrm{C}-\mathrm{NMR}, \mathrm{CDCl}_{3}$

C13CPD12hrs.d CDC13 \{C: \Bruker $\backslash$ Topspin3.2\} ALC 5

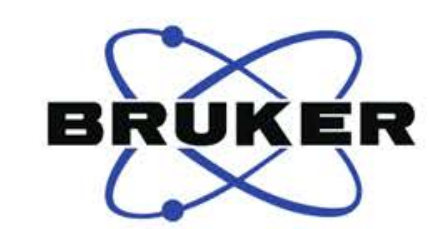

$\sum_{\substack{N \\ 1 \\ \mathrm{Boc} \\ 4 \mathrm{a}}}^{\mathrm{CO}} \mathrm{CO}_{2} \mathrm{Bn}$

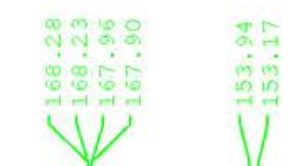

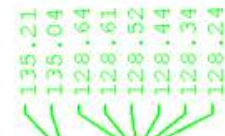

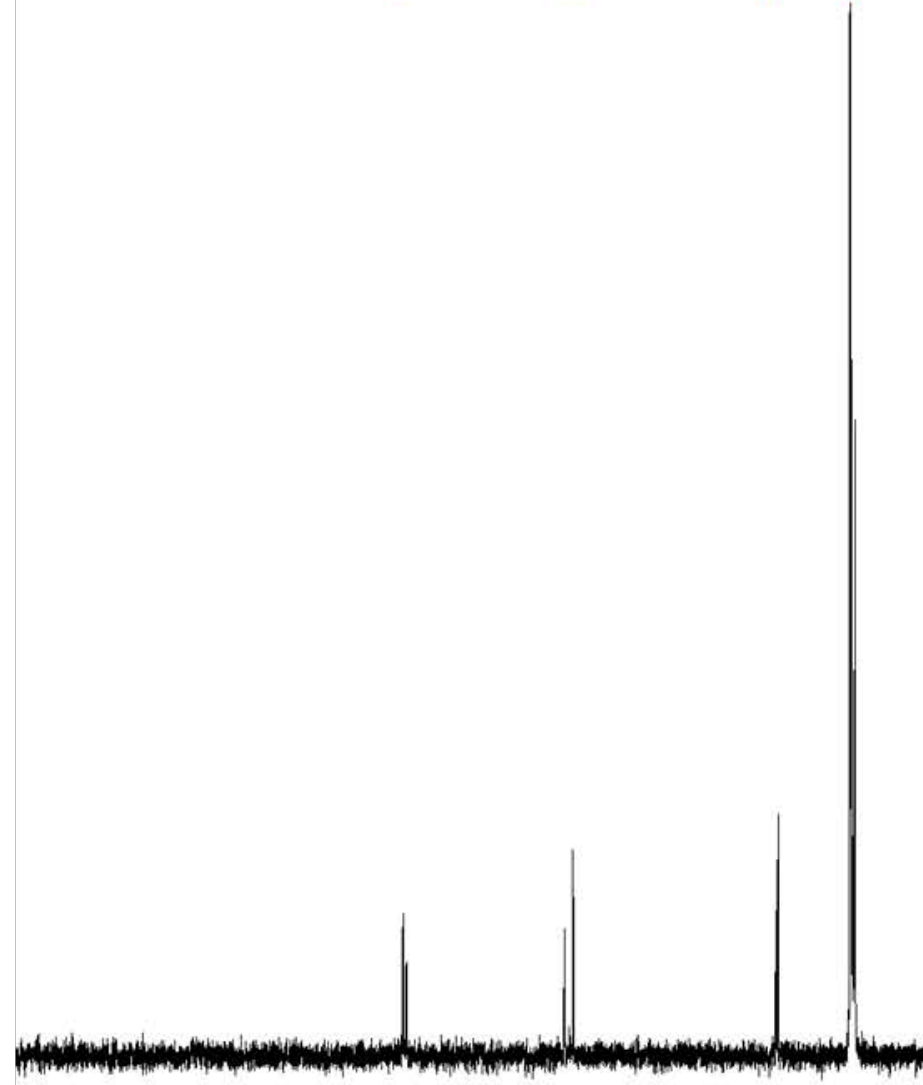



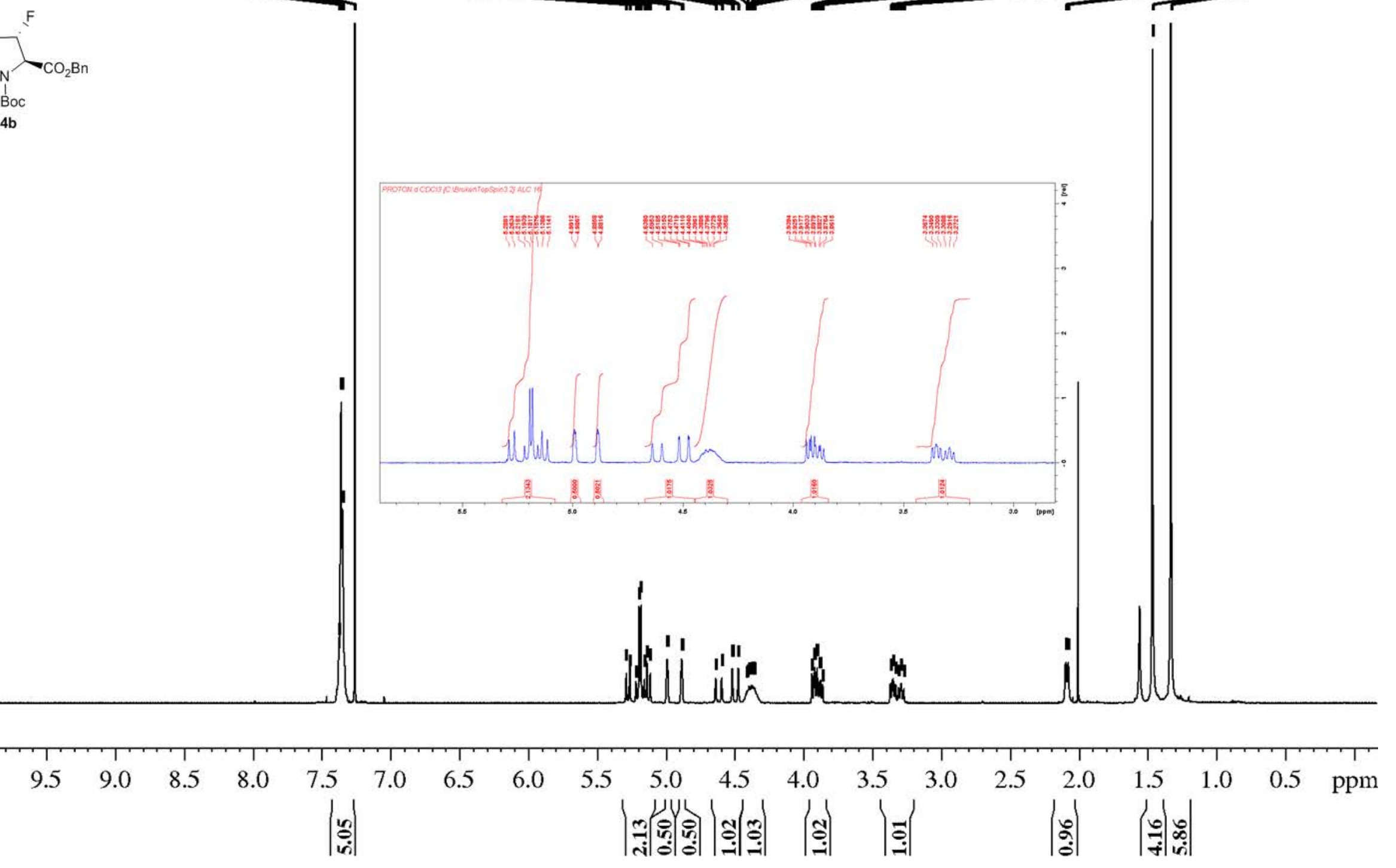
${ }^{19} \mathrm{~F}-\mathrm{NMR}, \mathrm{CDCl}_{3}$

19CPD.d CDCl3 \{C: \Bruker\TopSpin3.2\} ALC 16<smiles></smiles>

expinter

$\mathrm{CO}_{2} \mathrm{Bn}$

$\longrightarrow$

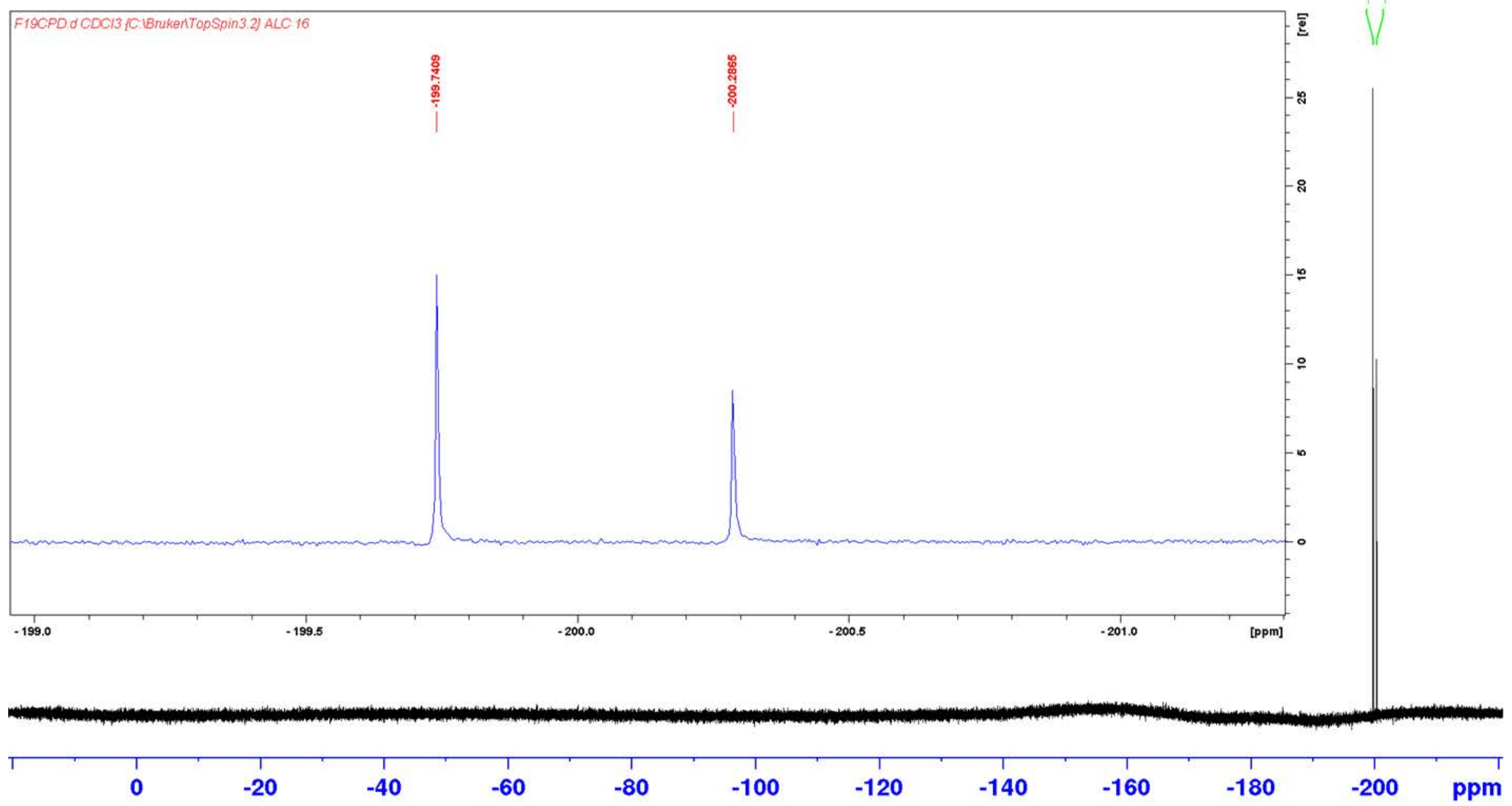


${ }^{13} \mathrm{C}-\mathrm{NMR}, \mathrm{CDCl}_{3}$
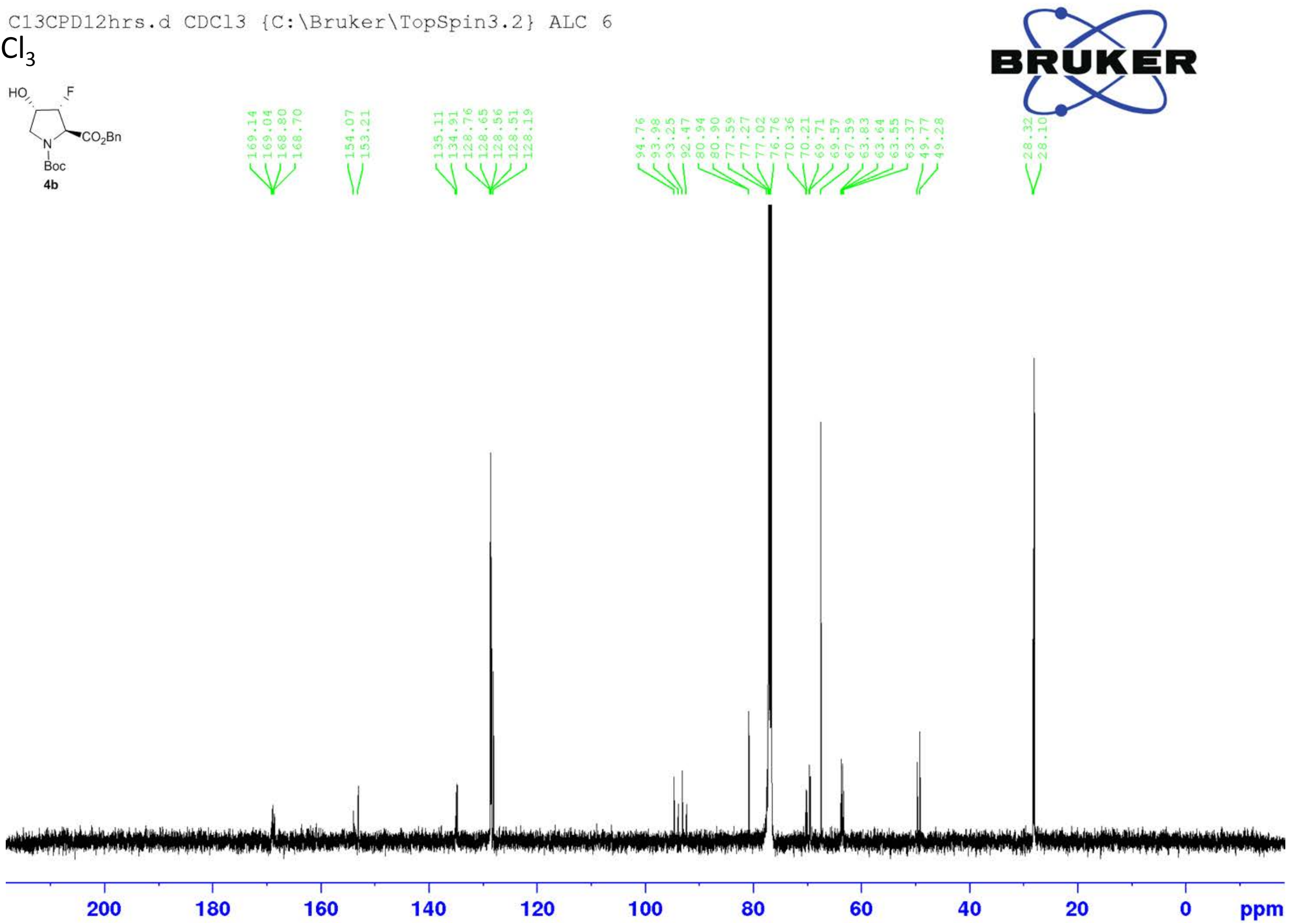


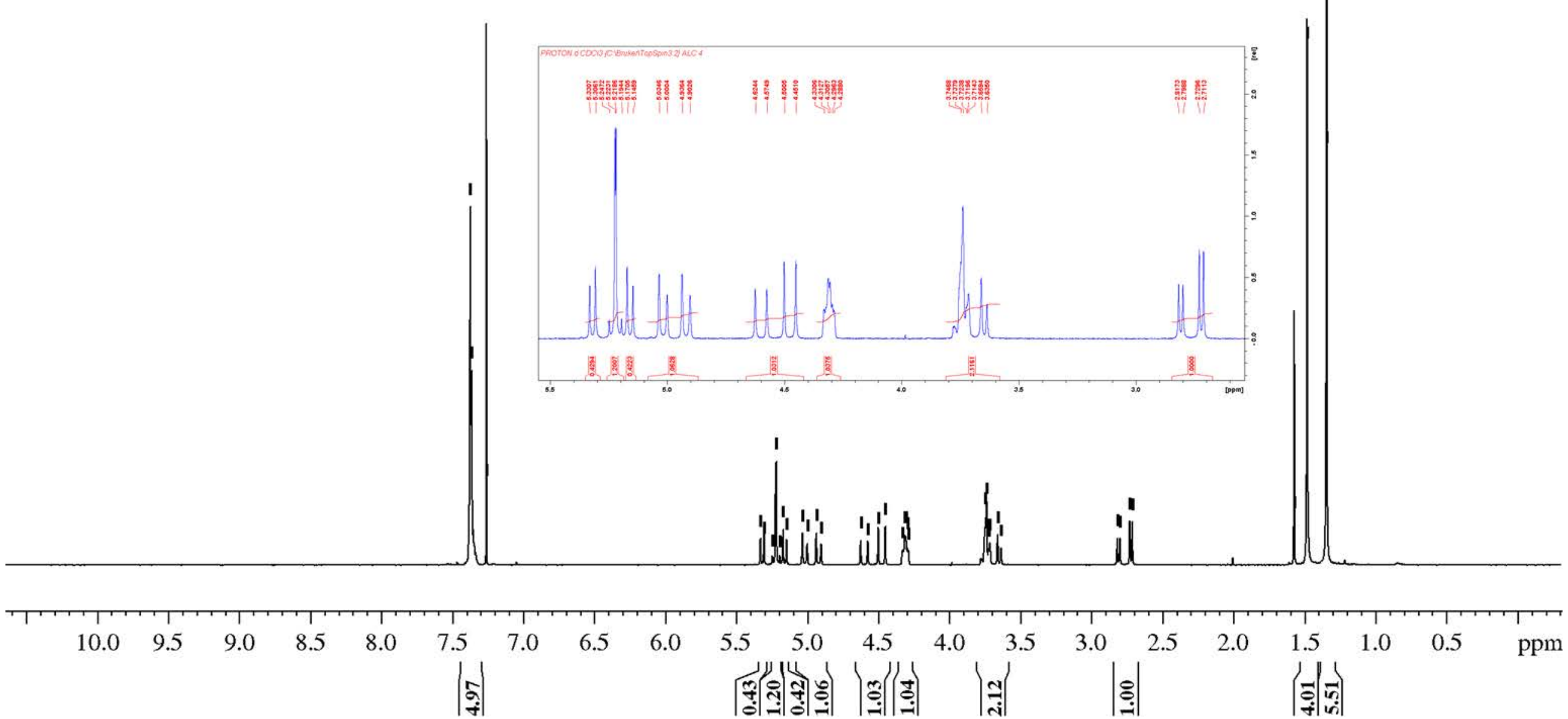




\section{${ }^{19} \mathrm{~F}-\mathrm{NMR}, \mathrm{CDCl}_{3}$}

19CPD.d CDC13 \{C: \Bruker \TopSpin3.2\} ALC 4
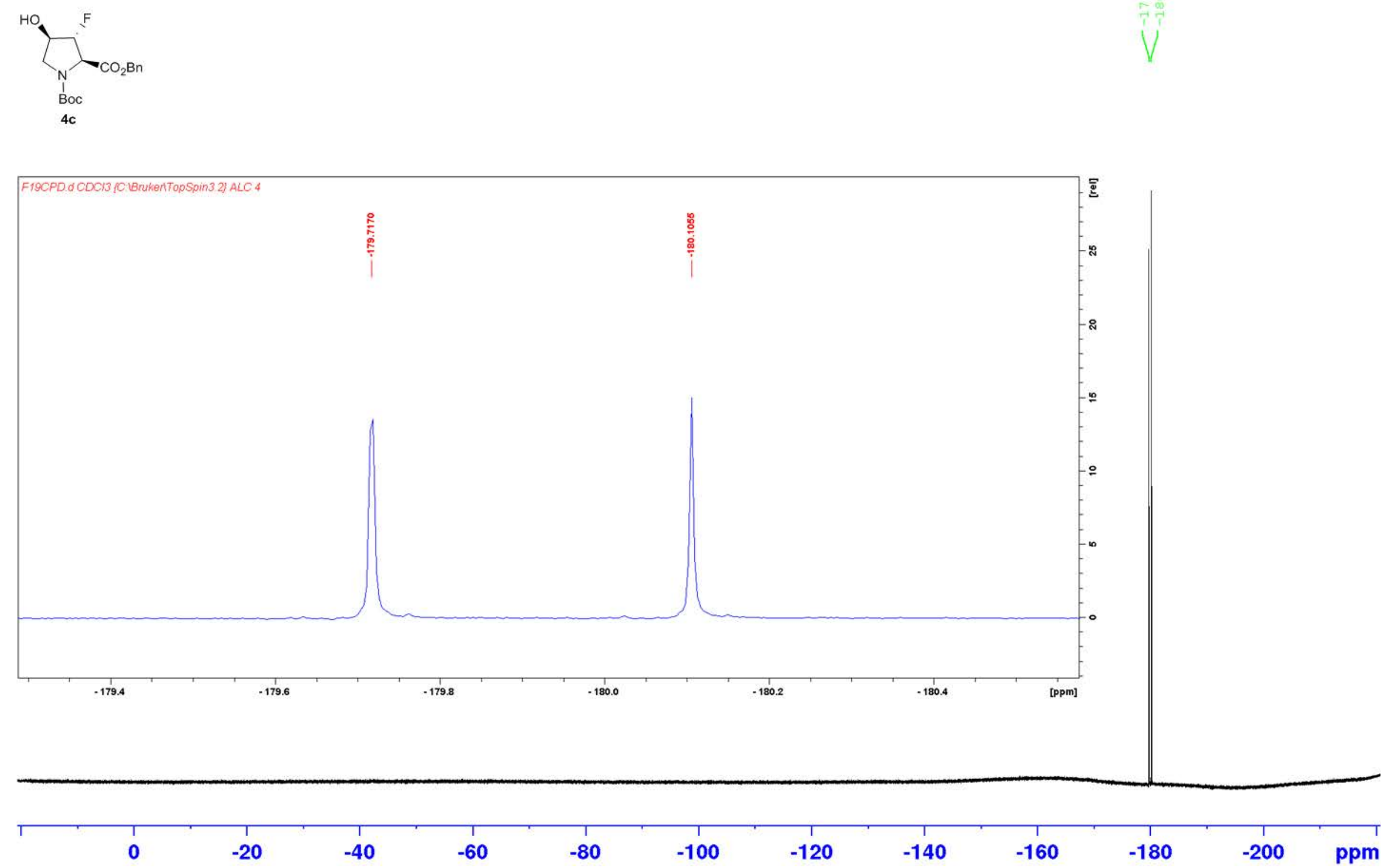
${ }^{13} \mathrm{C}-\mathrm{NMR}, \mathrm{CDCl}_{3}$

C13CPD6.d CDCl3 \{C: \Bruker \TopSpin3.2\} ALC 4

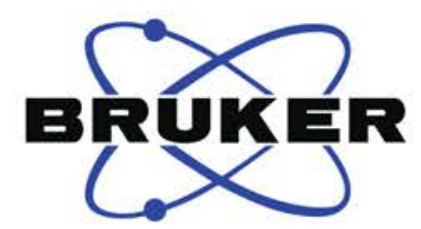

$\sum_{\substack{\mathrm{N} \\ \mathrm{Boc} \\ 4 \mathrm{c}}}^{\mathrm{CO}} \mathrm{CO}_{2} \mathrm{Bn}$

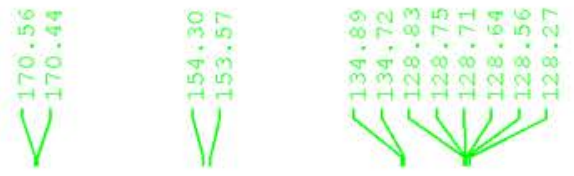

की

$\mid$

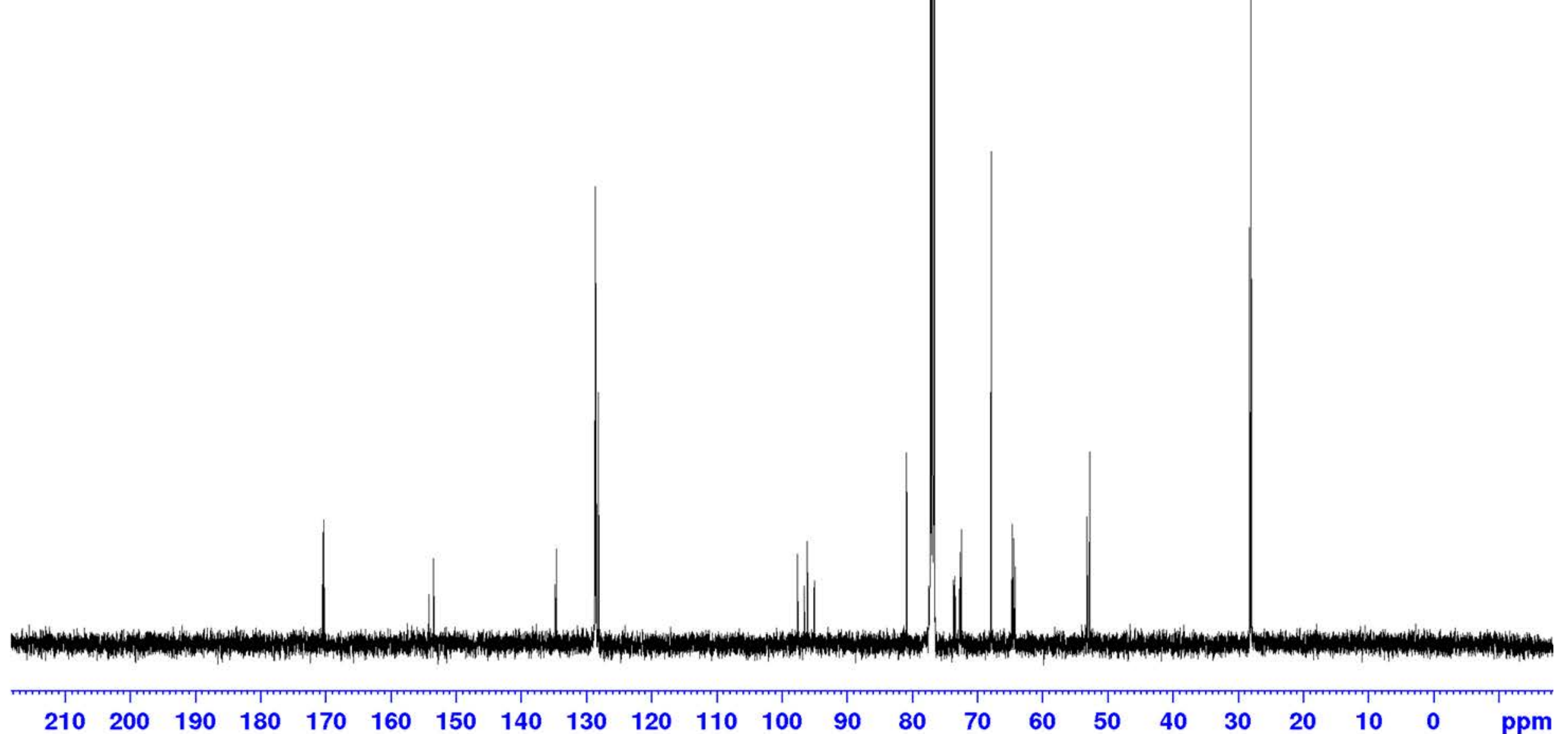


${ }^{1} \mathrm{H}-\mathrm{NMR}, \mathrm{CDCl}_{3}$

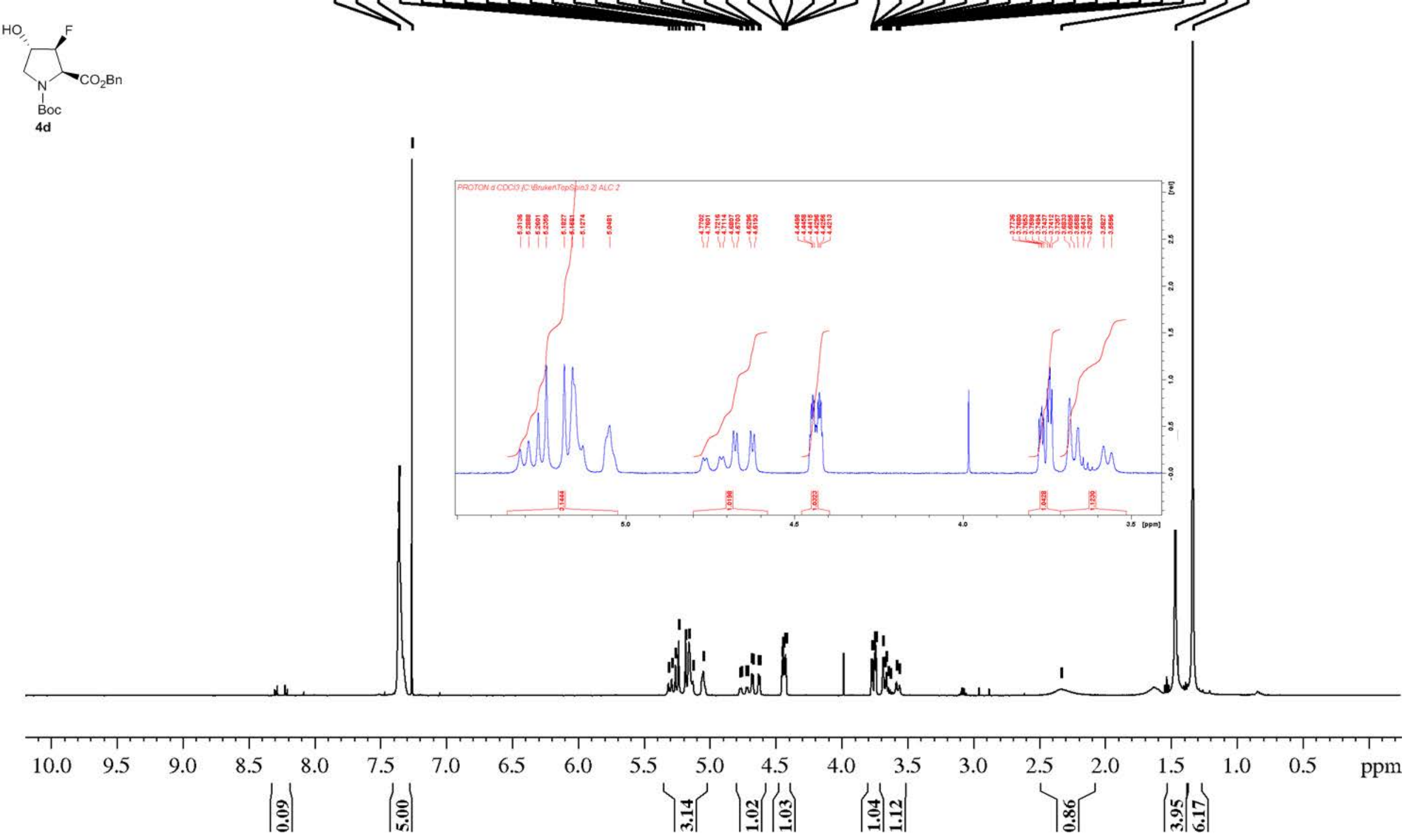


${ }^{19} \mathrm{~F}-\mathrm{NMR}, \mathrm{CDCl}_{3}$

F19CPD.d CDC13 \{C: \Bruker \TopSpin3.2\} ALC 2

\section{arfingen \\ CX}

רoc

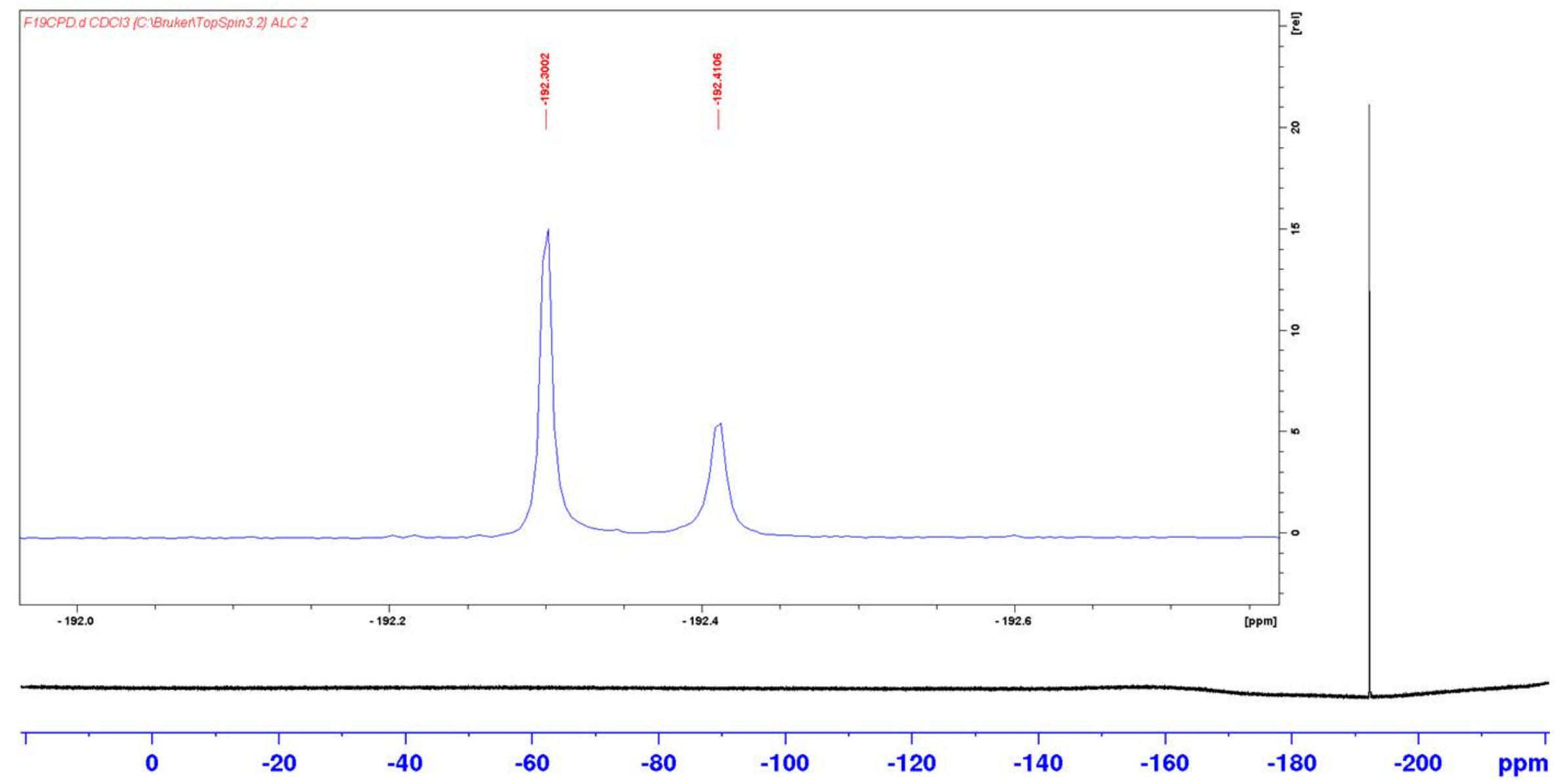


${ }^{13} \mathrm{C}-\mathrm{NMR}, \mathrm{CDCl}{ }_{3}$

$\mathrm{HO}$
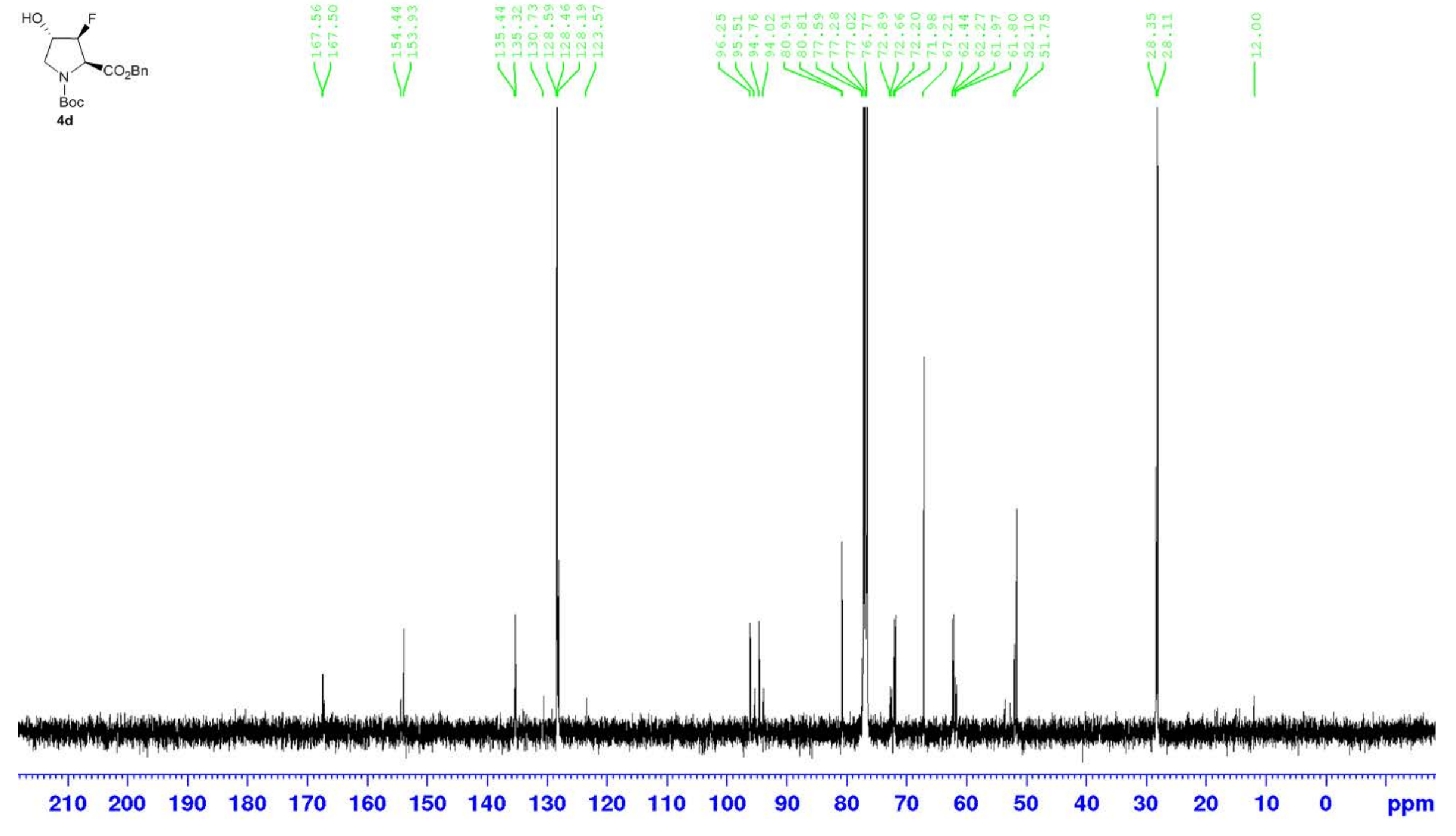


\section{BRUKER \\ C}

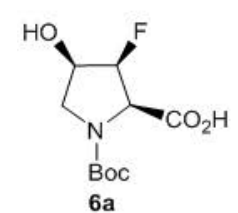

$6 a$

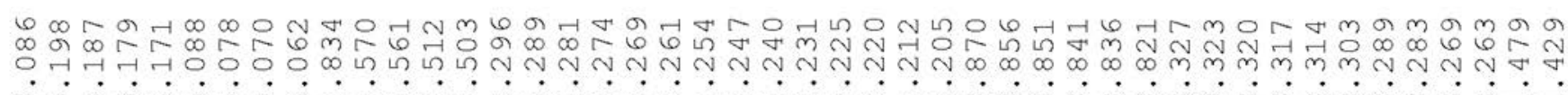
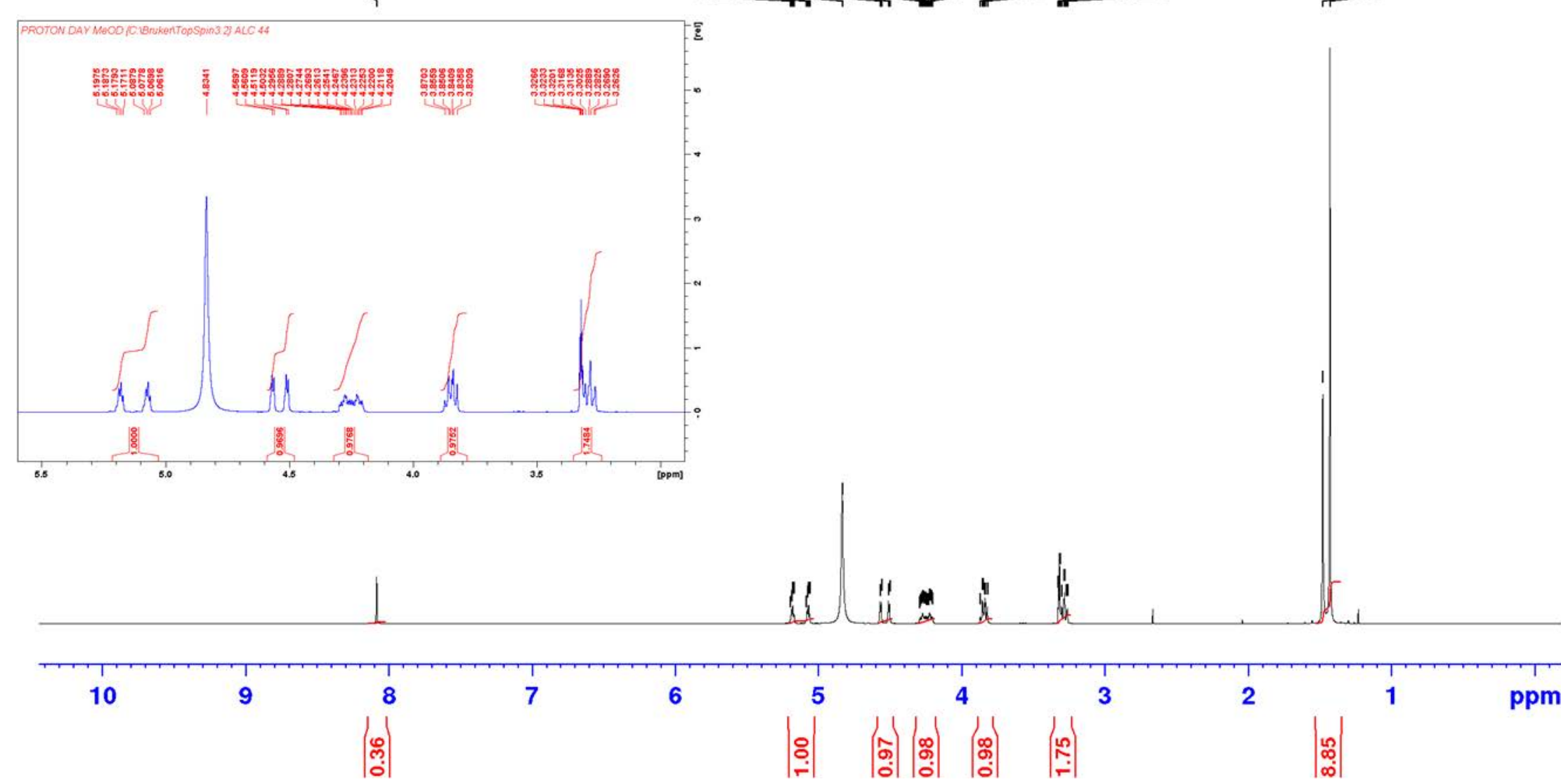


\section{exprenter \\ C X}

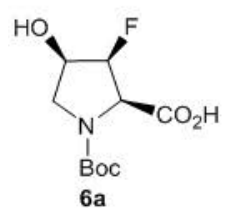

Bac
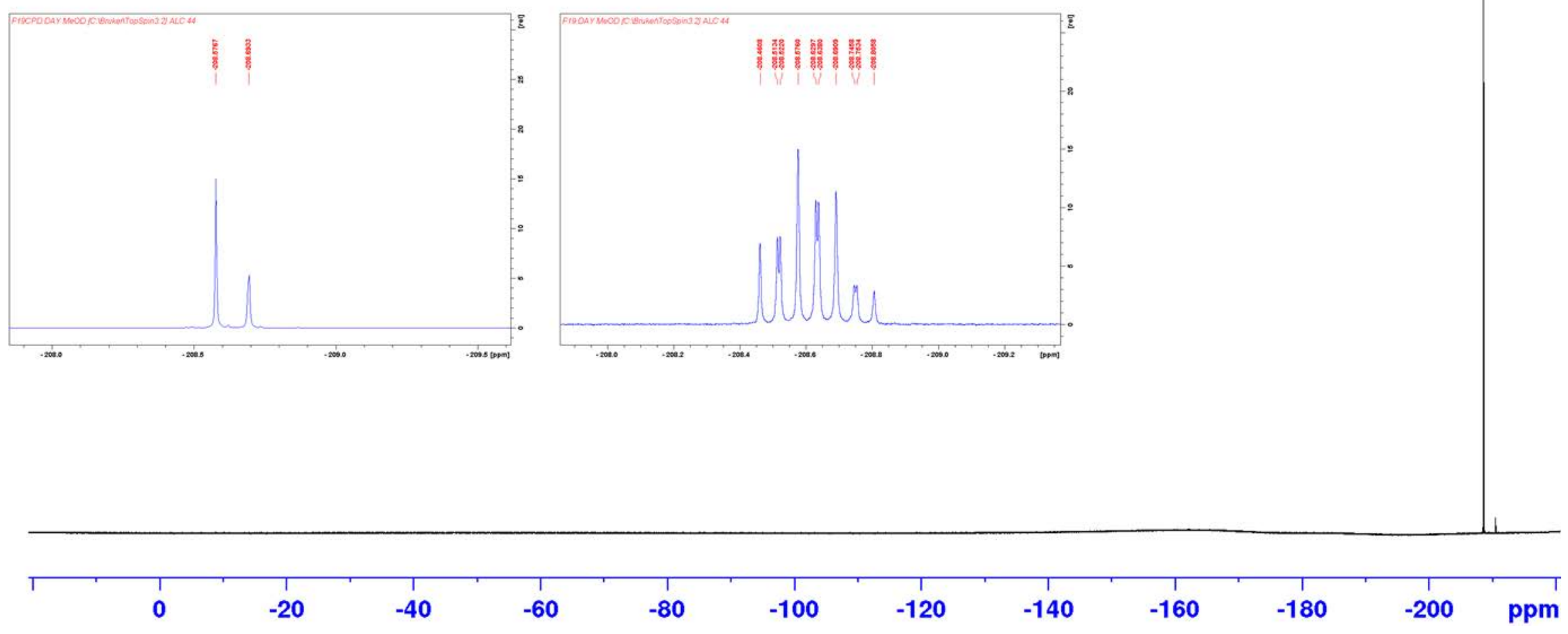

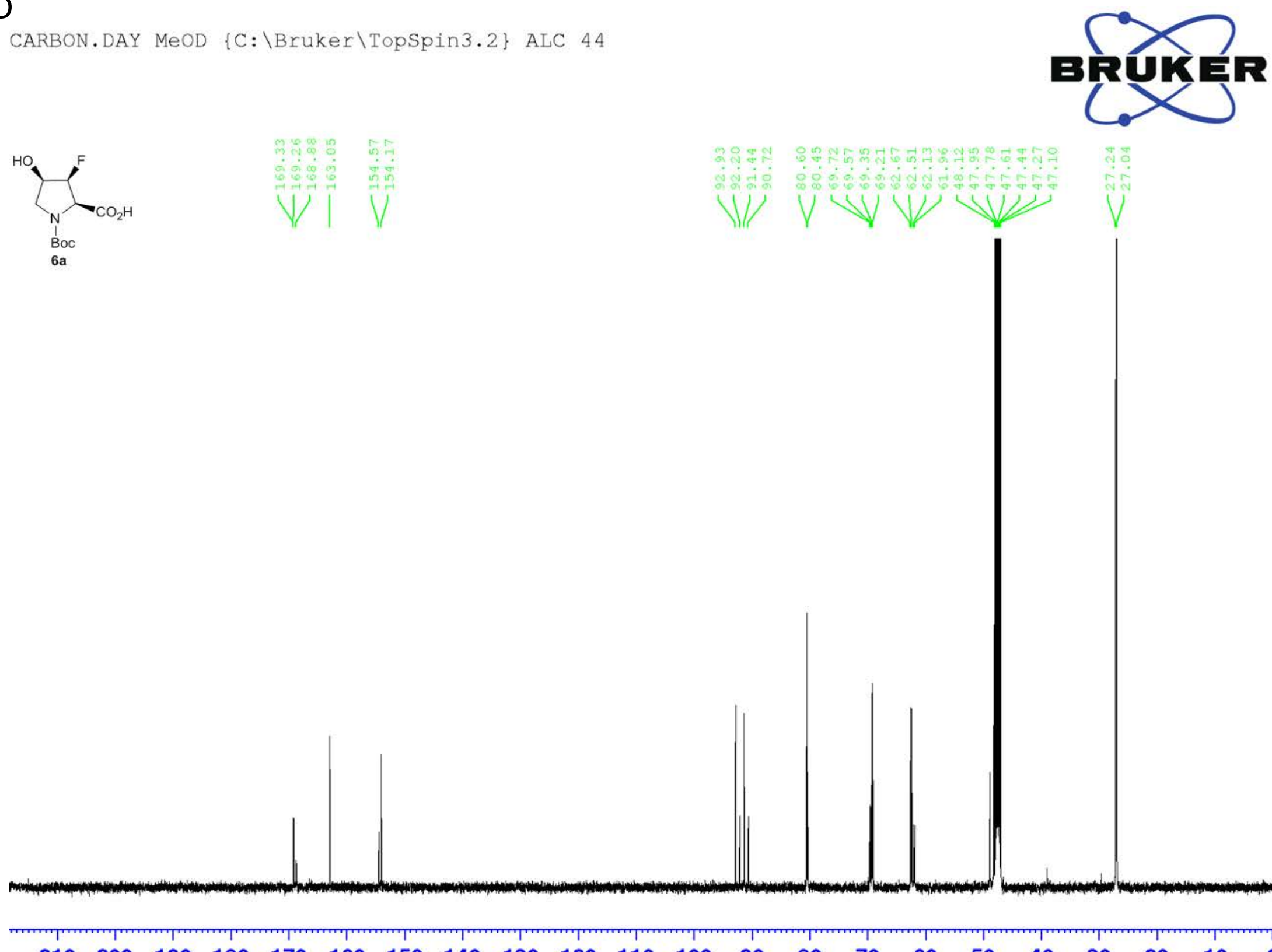

8070 


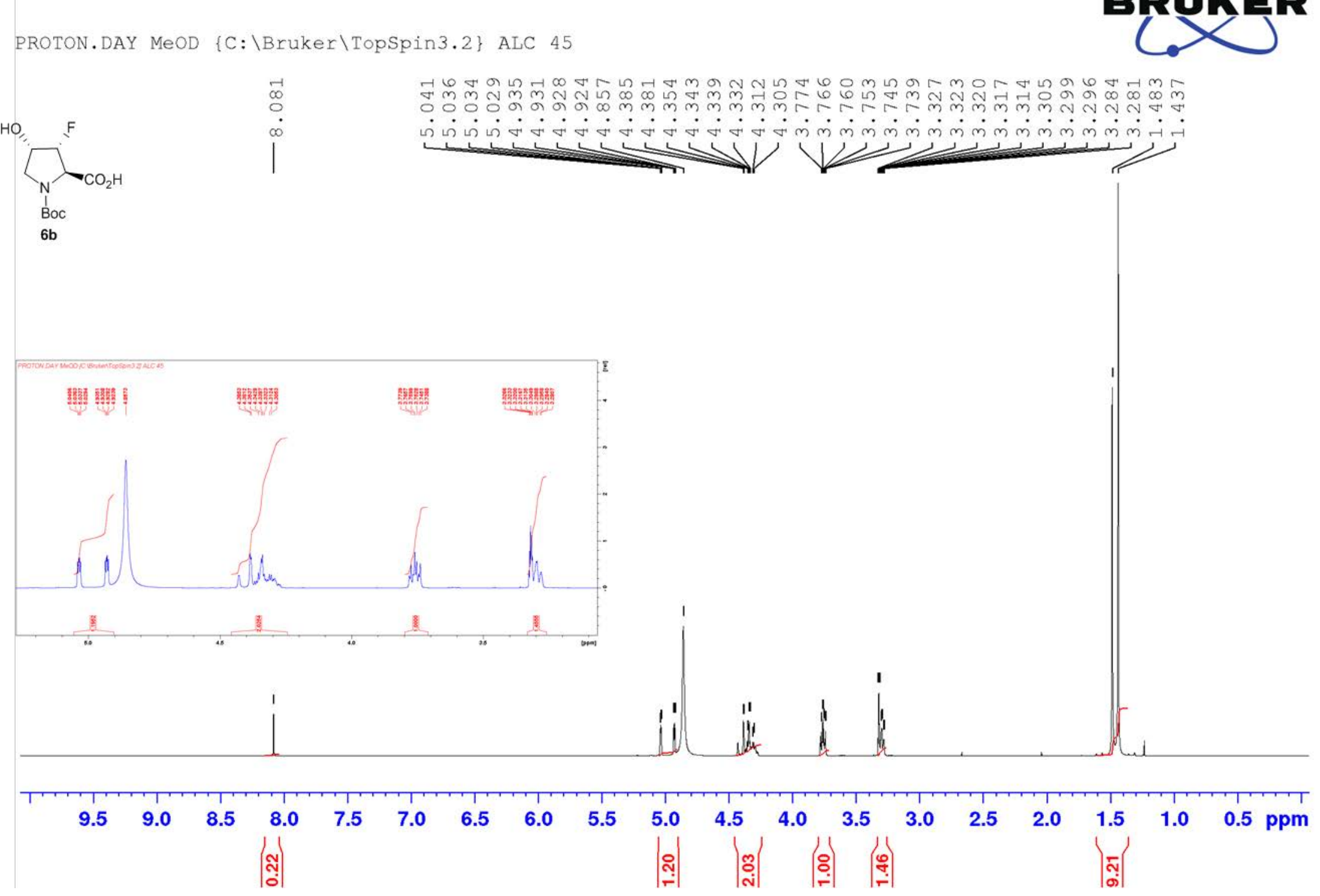


${ }^{19} \mathrm{~F}-\mathrm{NMR}, \mathrm{CD}_{3} \mathrm{OD}$

F19CPD.DAY MeOD \{C: \Bruker \TopSpin3.2\} ALC 56

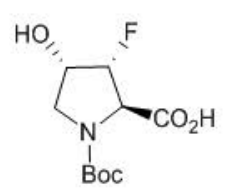

$6 b$

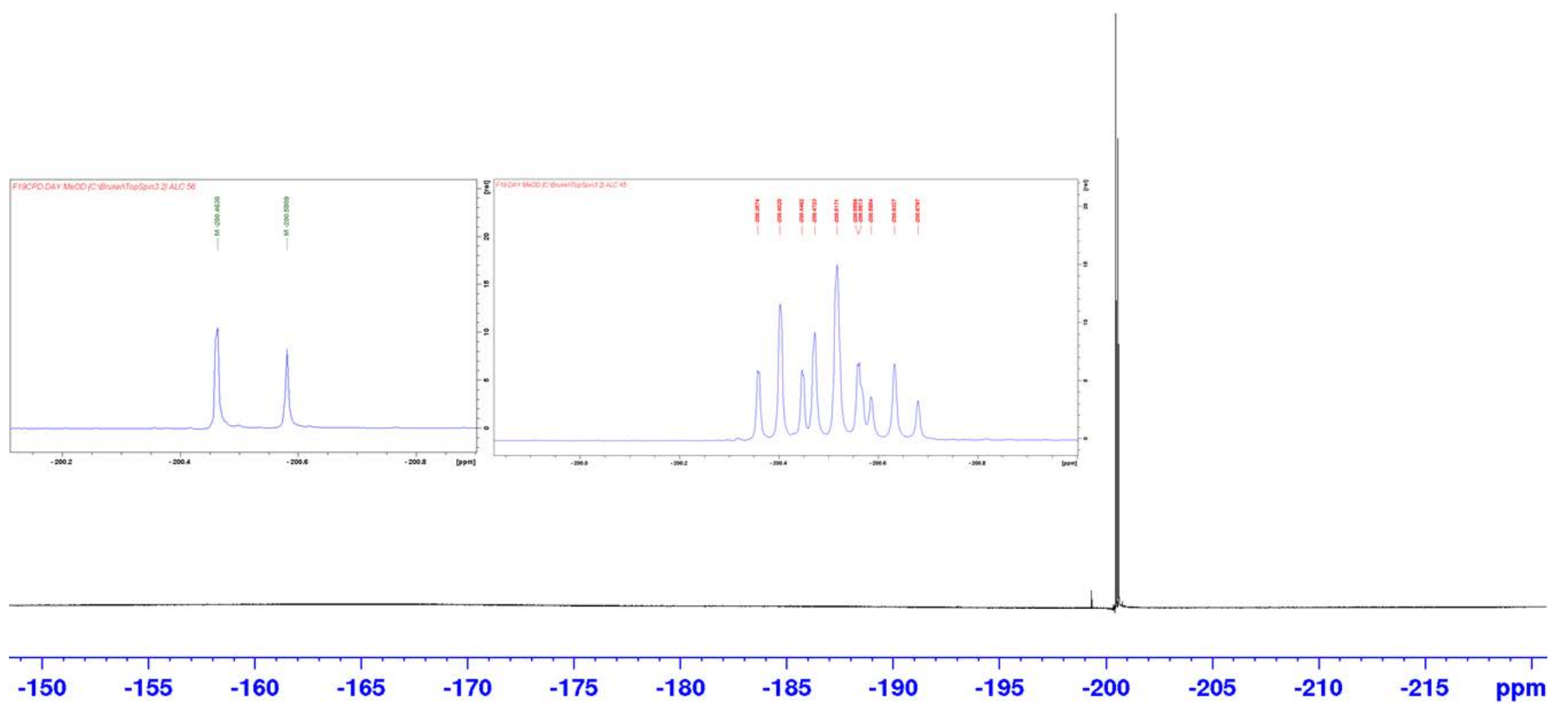


${ }^{13} \mathrm{C}-\mathrm{NMR}, \mathrm{CD}_{3} \mathrm{OD}$

CARBON.DAY MeOD \{C: \Bruker \Topspin3.2\} ALC 45

\section{BRUKER \\ C $>$}

$\sum_{\substack{\mathrm{Noc} \\ \mathrm{Boc} \\ 6 \mathrm{~b}}}^{\mathrm{HO}}$

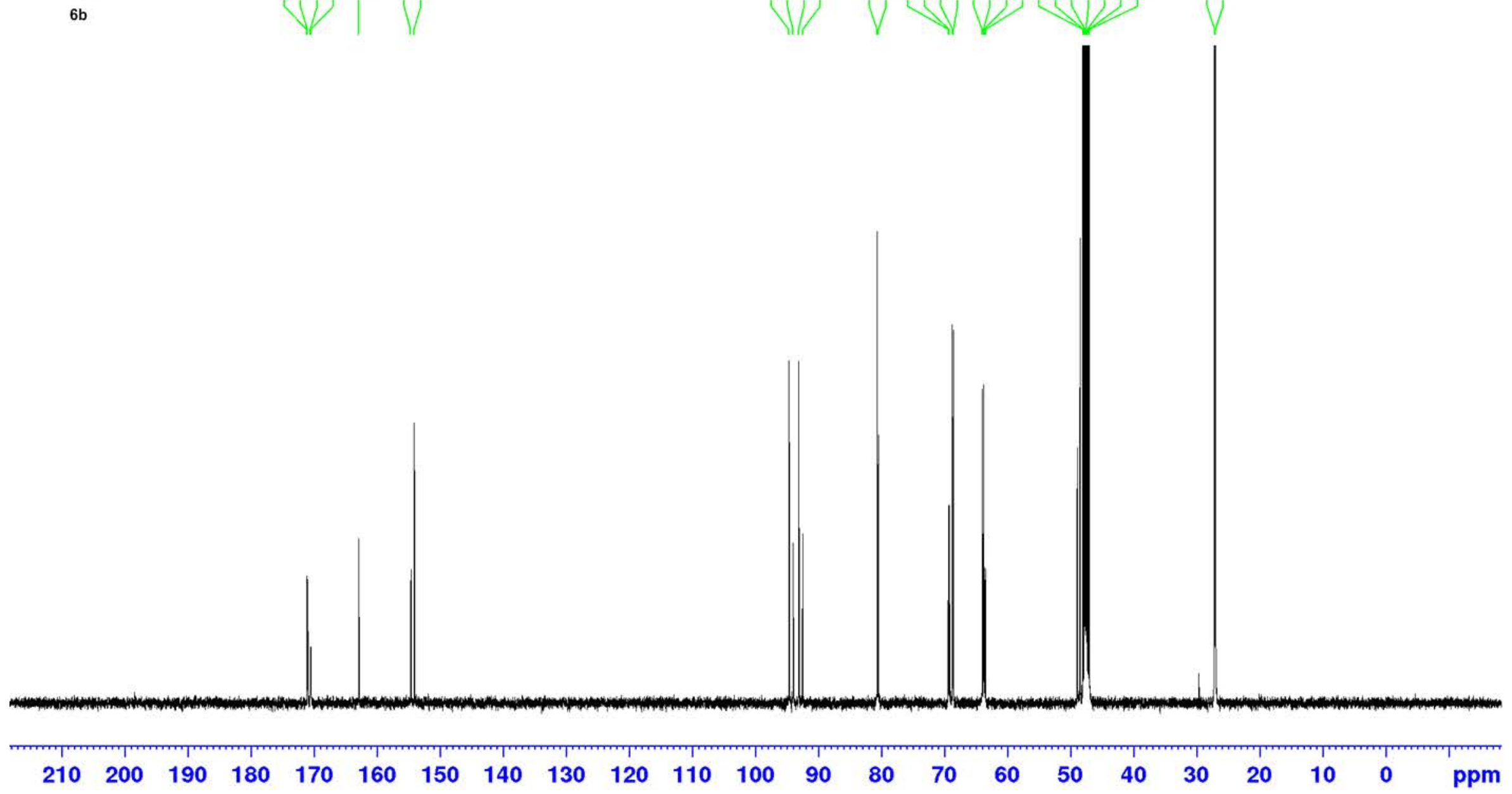




\section{GrukER}

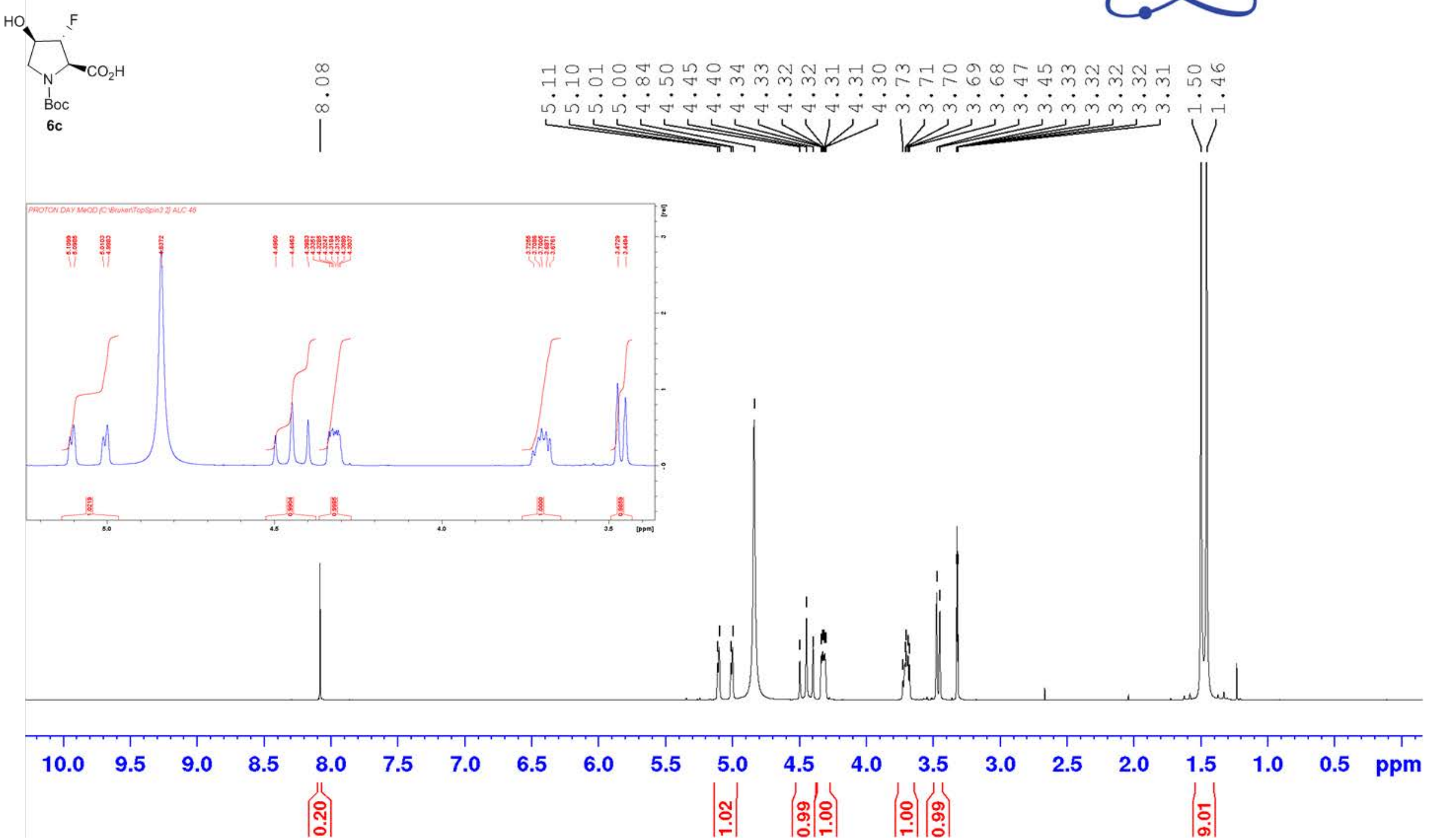


${ }^{19} \mathrm{~F}-\mathrm{NMR}, \mathrm{CD}_{3} \mathrm{OD}$

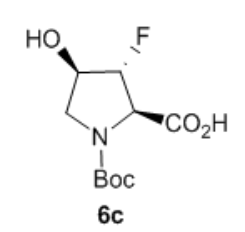

E19CPD.DAY MeOD \{C: \Bruker $\backslash$ TopSpin3.2\} ALC 46

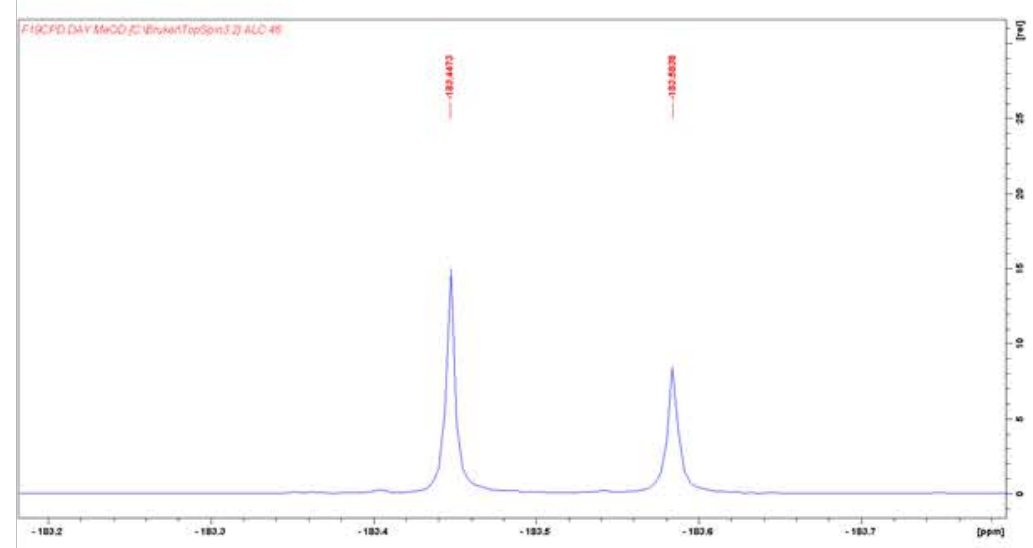

arifing
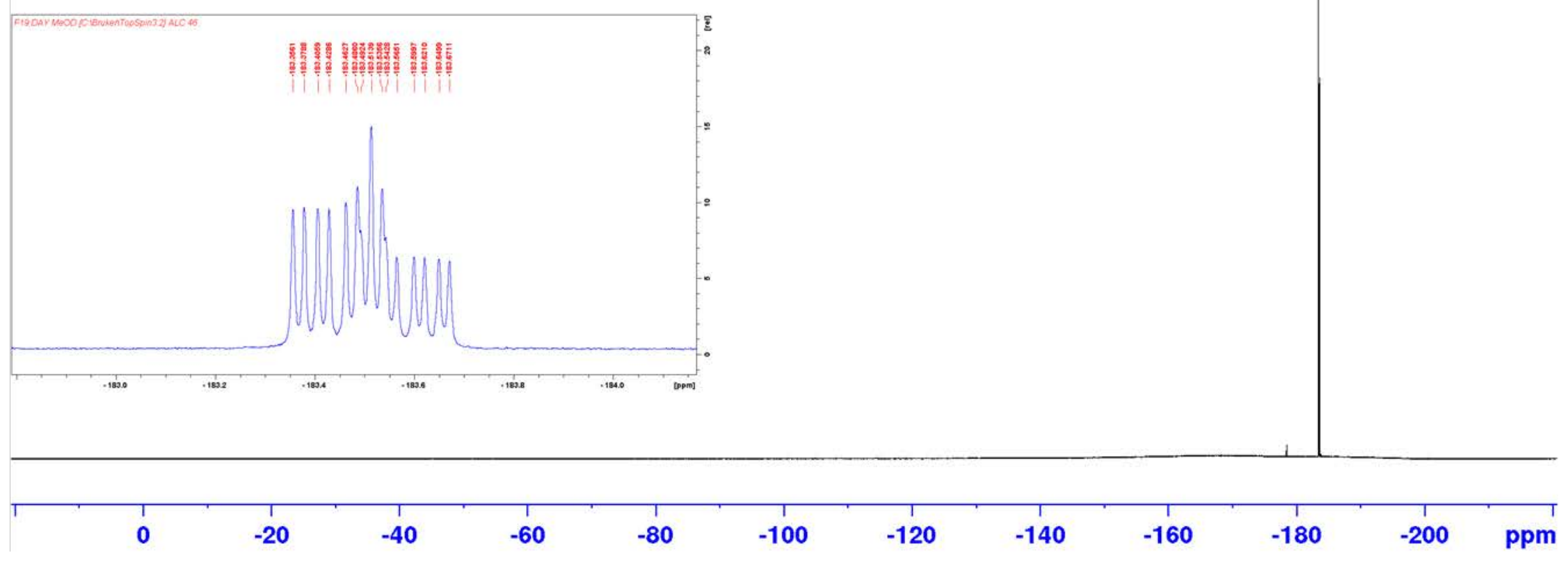


\section{BRUKER}
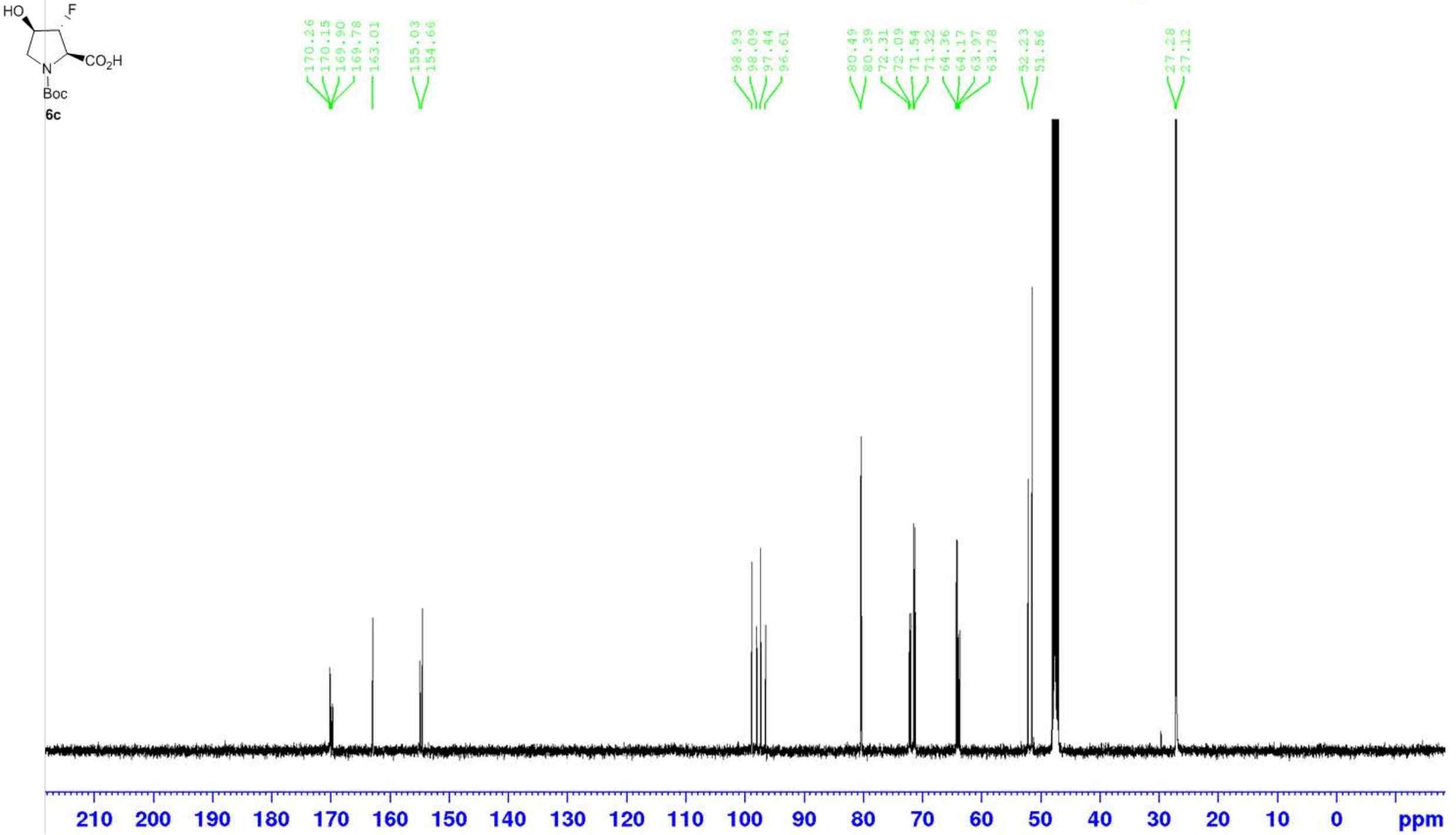
${ }^{1} \mathrm{H}-\mathrm{NMR}, \mathrm{CD}_{3} \mathrm{OD}$

PROTON.DAY MEOD \{C: \Bruker\TOpSpin3.2\} ALC 43

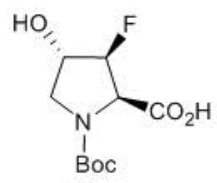

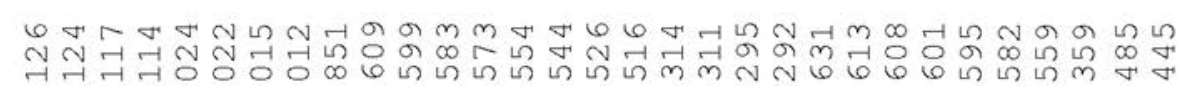

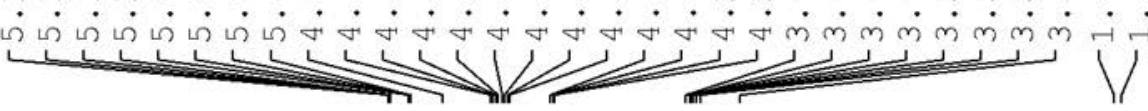

Boc

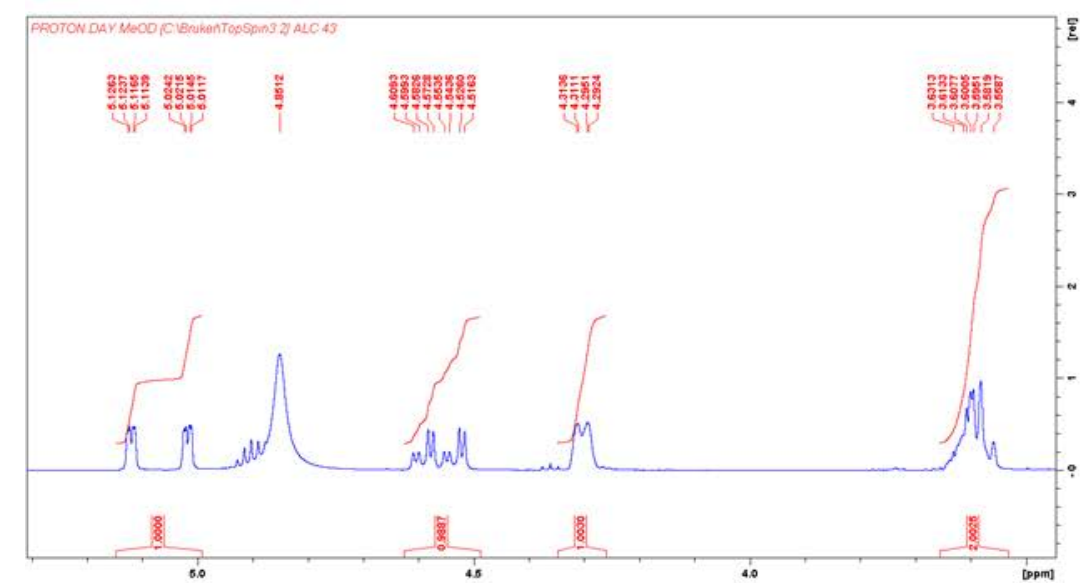

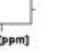

i) "is"

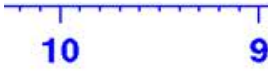

8
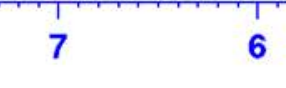

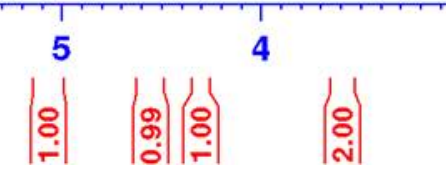

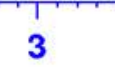

2

1

0 ppm

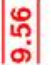


${ }^{19} \mathrm{~F}-\mathrm{NMR}, \mathrm{CD}_{3} \mathrm{OD}$

HO,

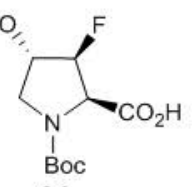

$\mathrm{C}$ Boc
$6 d$
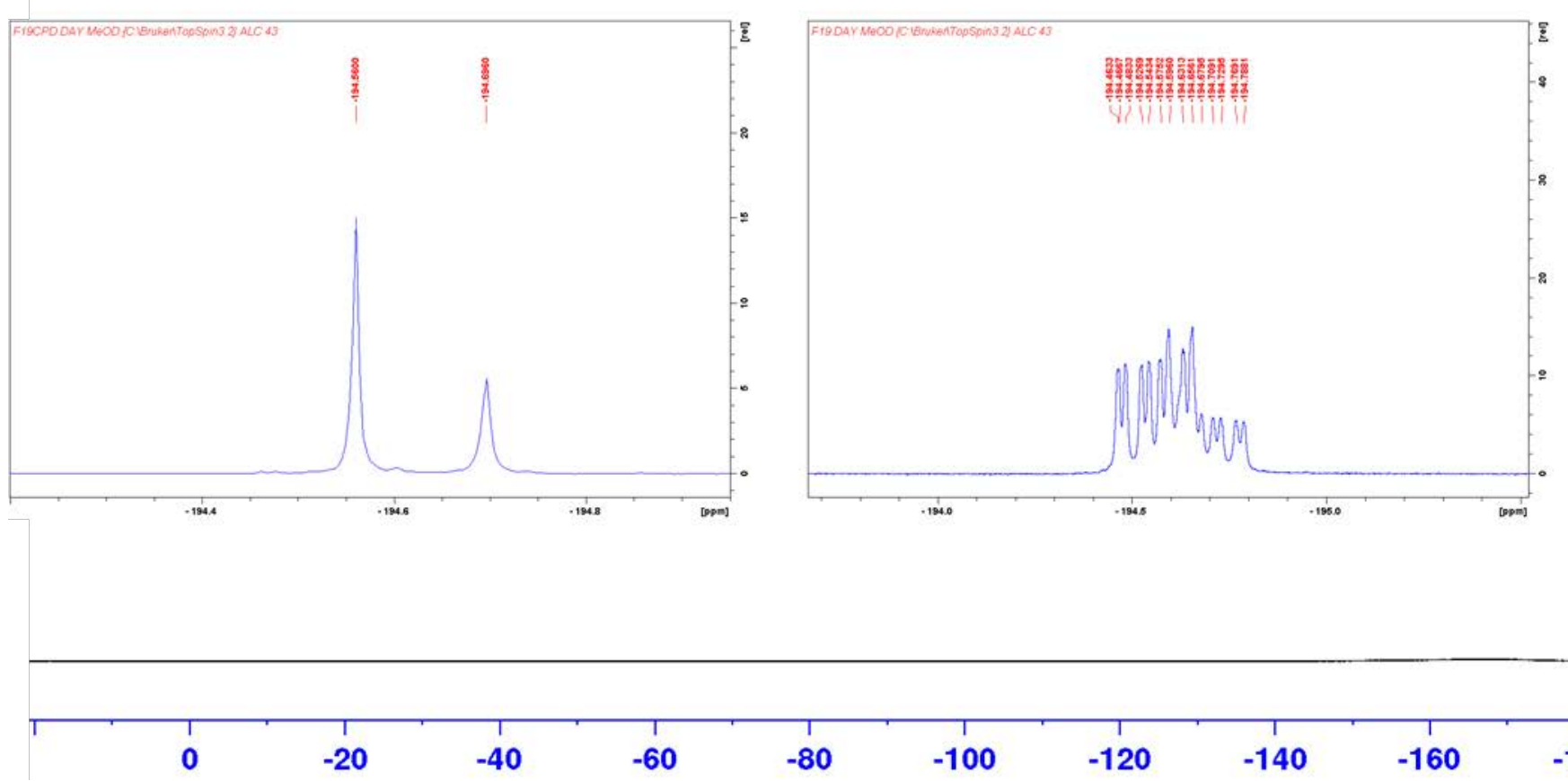

$-80$

$-100$

$-120$

$-140$

$-160$

$-180$

$-200$ 
${ }^{13} \mathrm{C}-\mathrm{NMR}, \mathrm{CD}_{3} \mathrm{OD}$

CARBON.DAY MeOD \{C: \Bruker\TopSpin3.2\} ALC 43
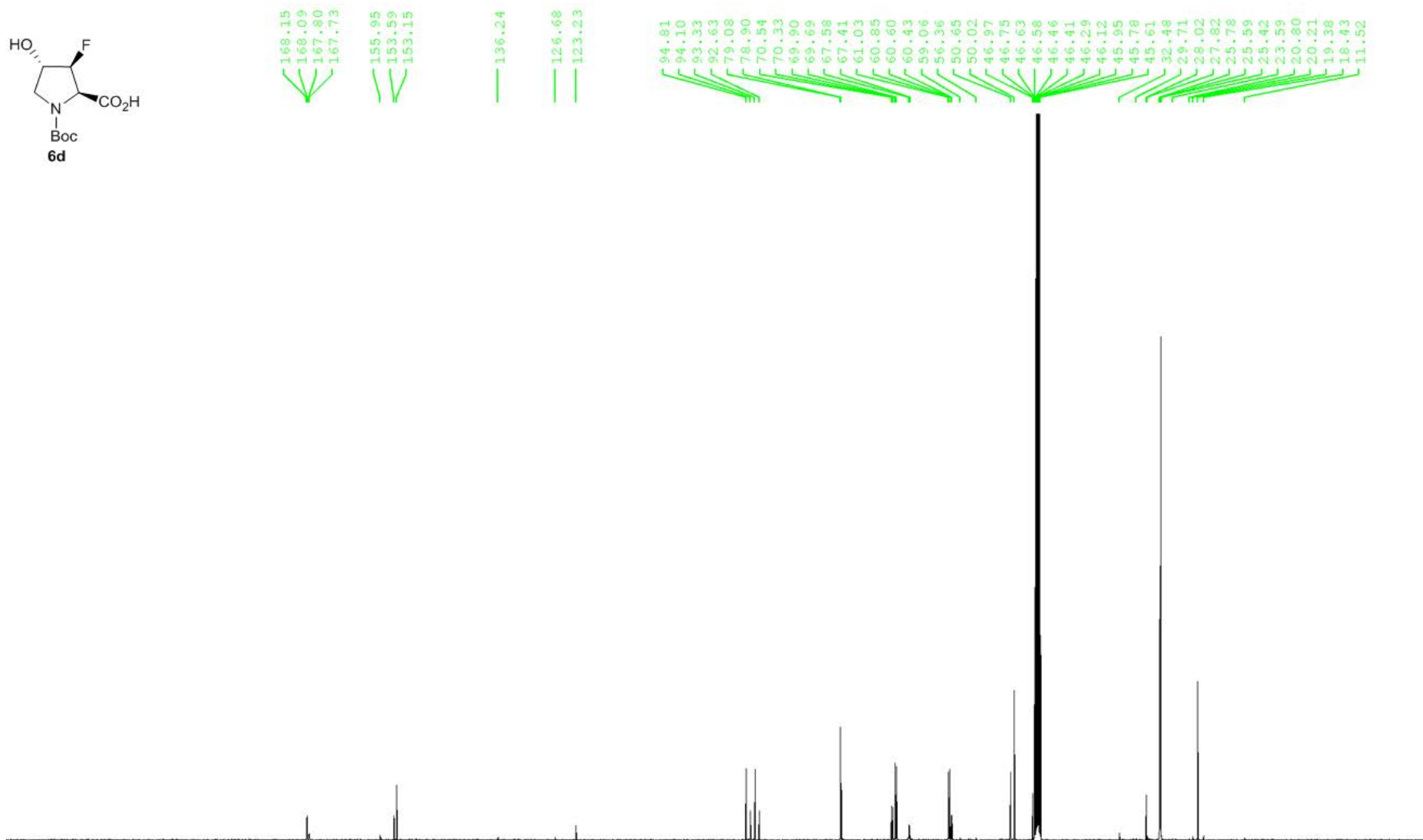

$\begin{array}{lllllllllllll}210 & 200 & 190 & 180 & 170 & 160 & 150 & 140 & 130 & 120 & 110 & 100 & 90\end{array}$ 
${ }^{1} \mathrm{H}-\mathrm{NMR}, \mathrm{CDCl}_{3}$

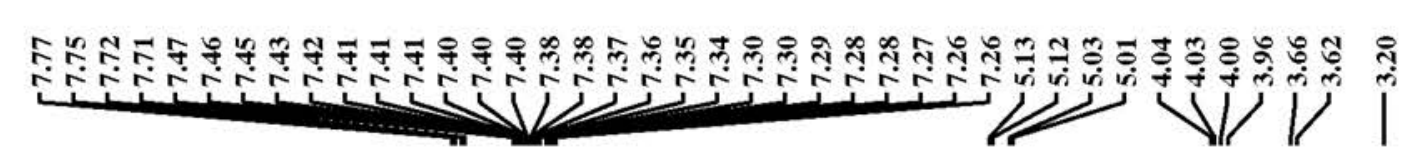

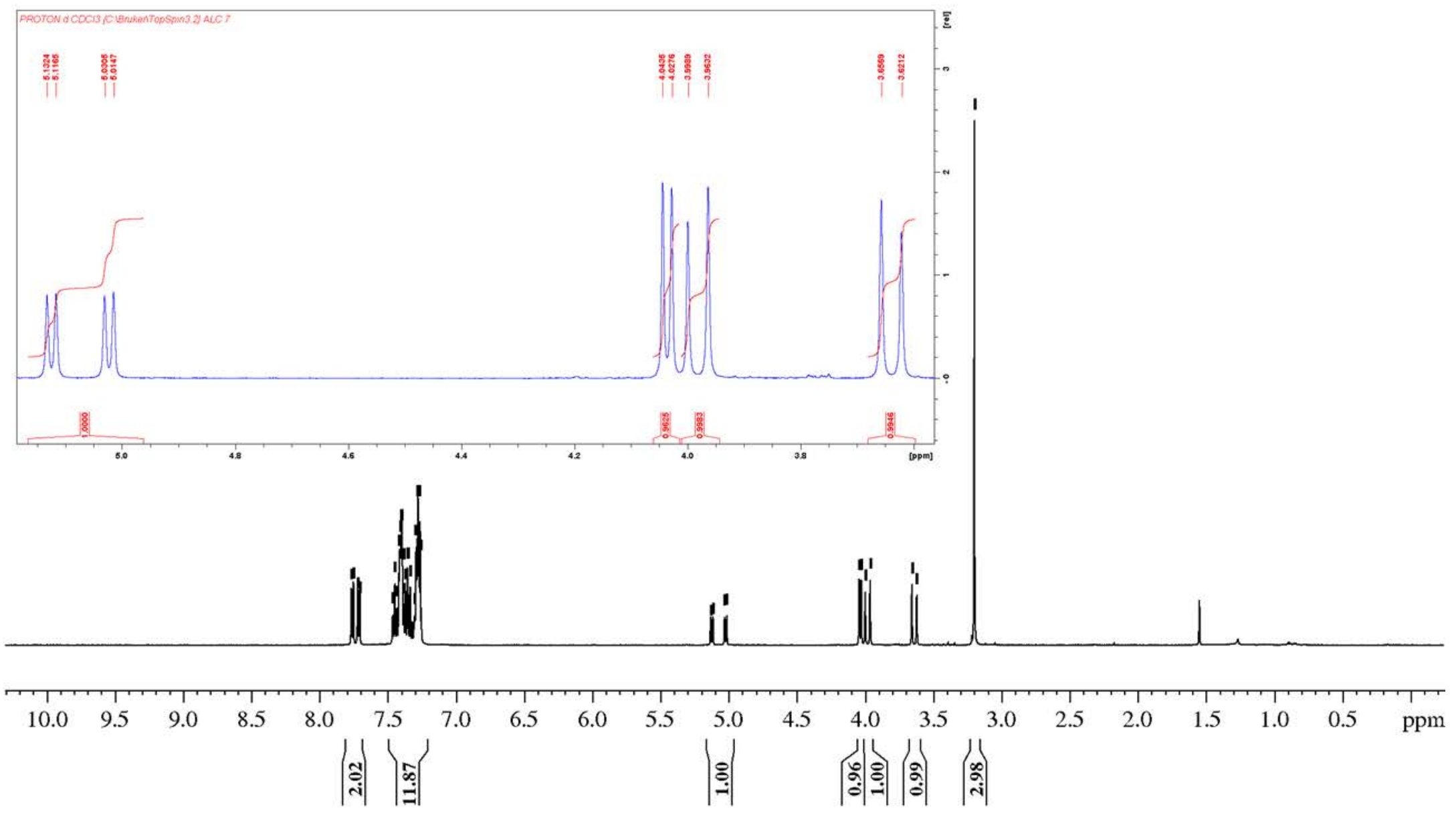


${ }^{19} \mathrm{~F}-\mathrm{NMR}, \mathrm{CDCl}_{3}$

F19CPD.d CDCl3 \{C: \Bruker \TopSpin3.2\} ALC 7

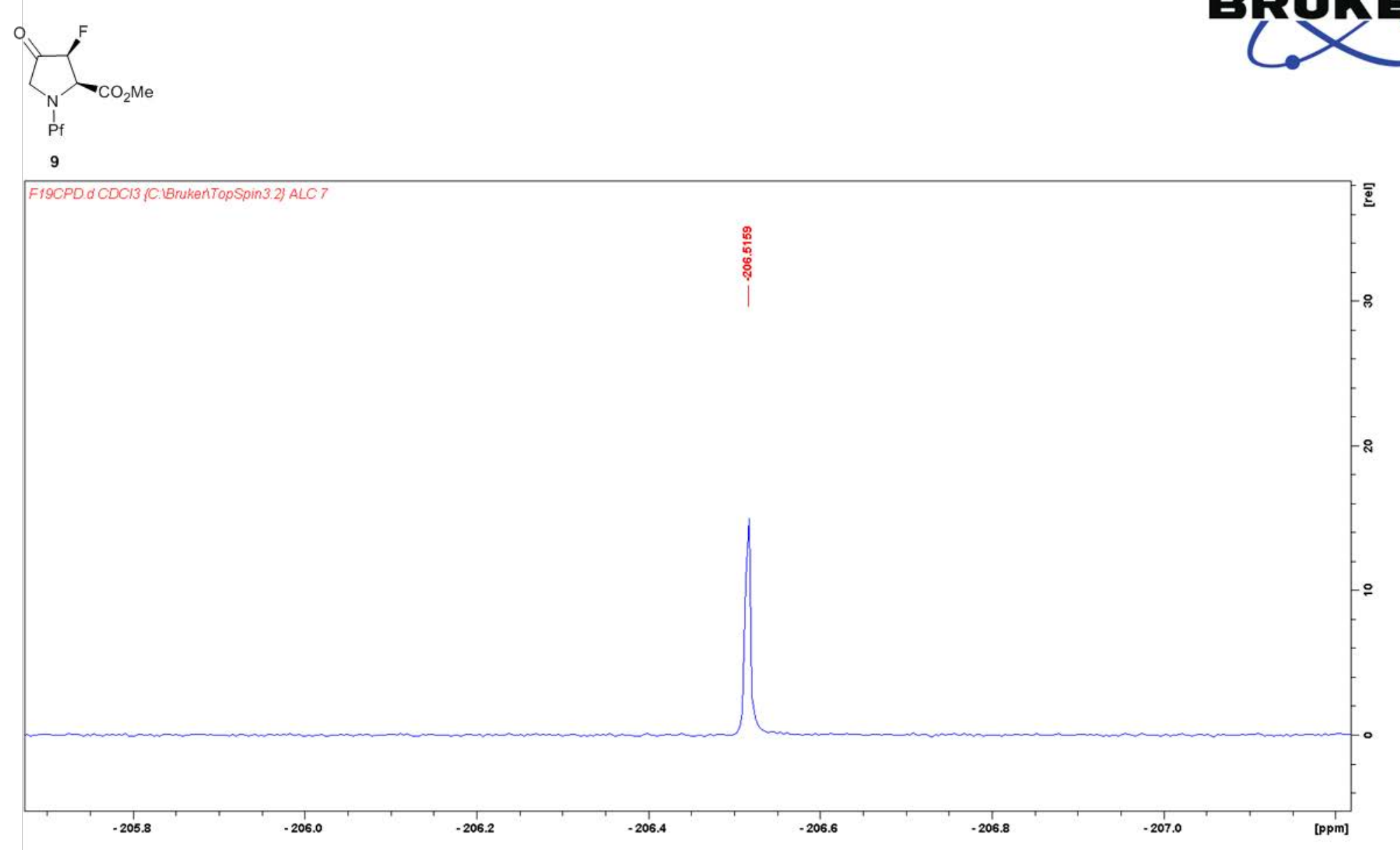

misigen

1
$\mathrm{Pf}$
$\mathrm{P}$

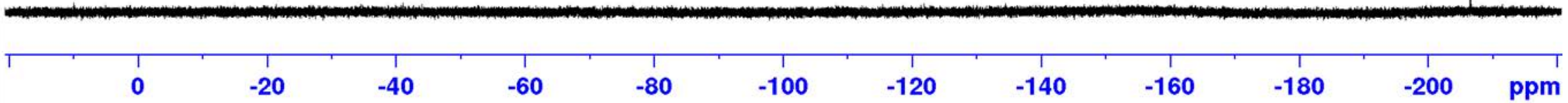




\section{${ }^{13} \mathrm{C}-\mathrm{NMR}, \mathrm{CDCl}_{3}$}

C13CPD6.d CDCl3 \{C: \Bruker \Topspin3.2\} ALC 2

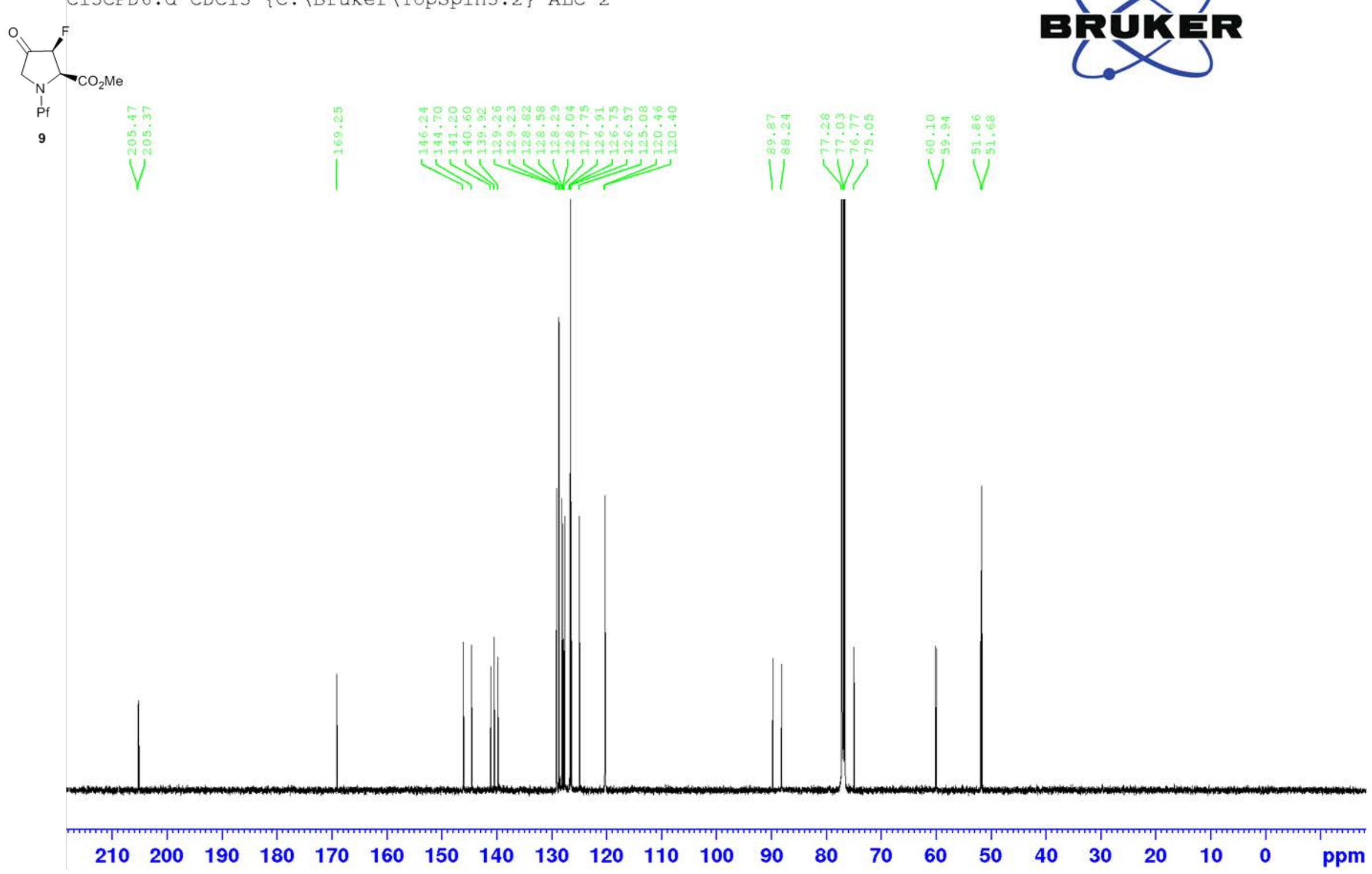


${ }^{1} \mathrm{H}-\mathrm{NMR}, \mathrm{CDCl}_{3}$

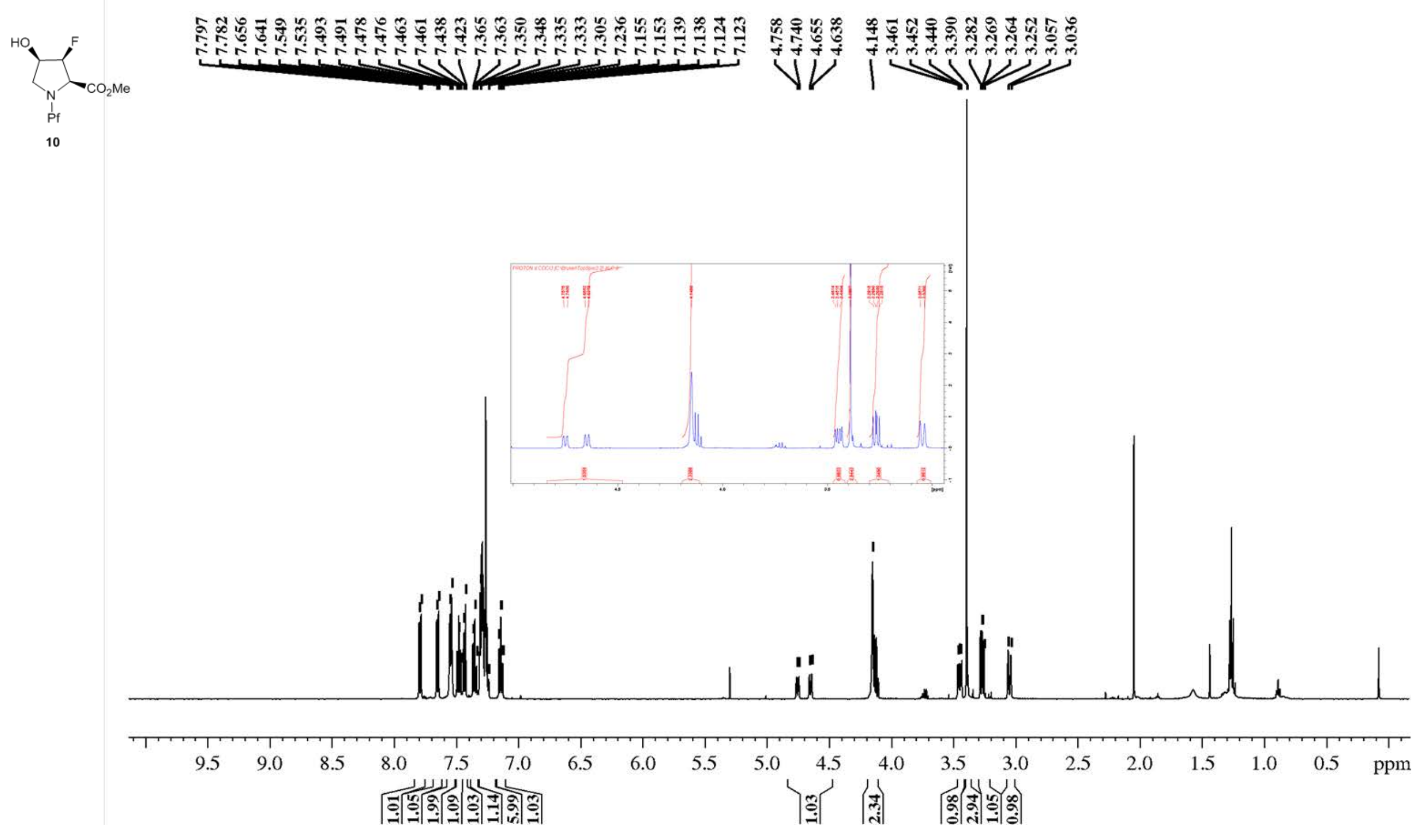


${ }^{19} \mathrm{~F}-\mathrm{NMR}, \mathrm{CDCl}_{3}$

F19CPD.d CDCl3 \{C: \Bruker \TopSpin3.2\} ALC 9

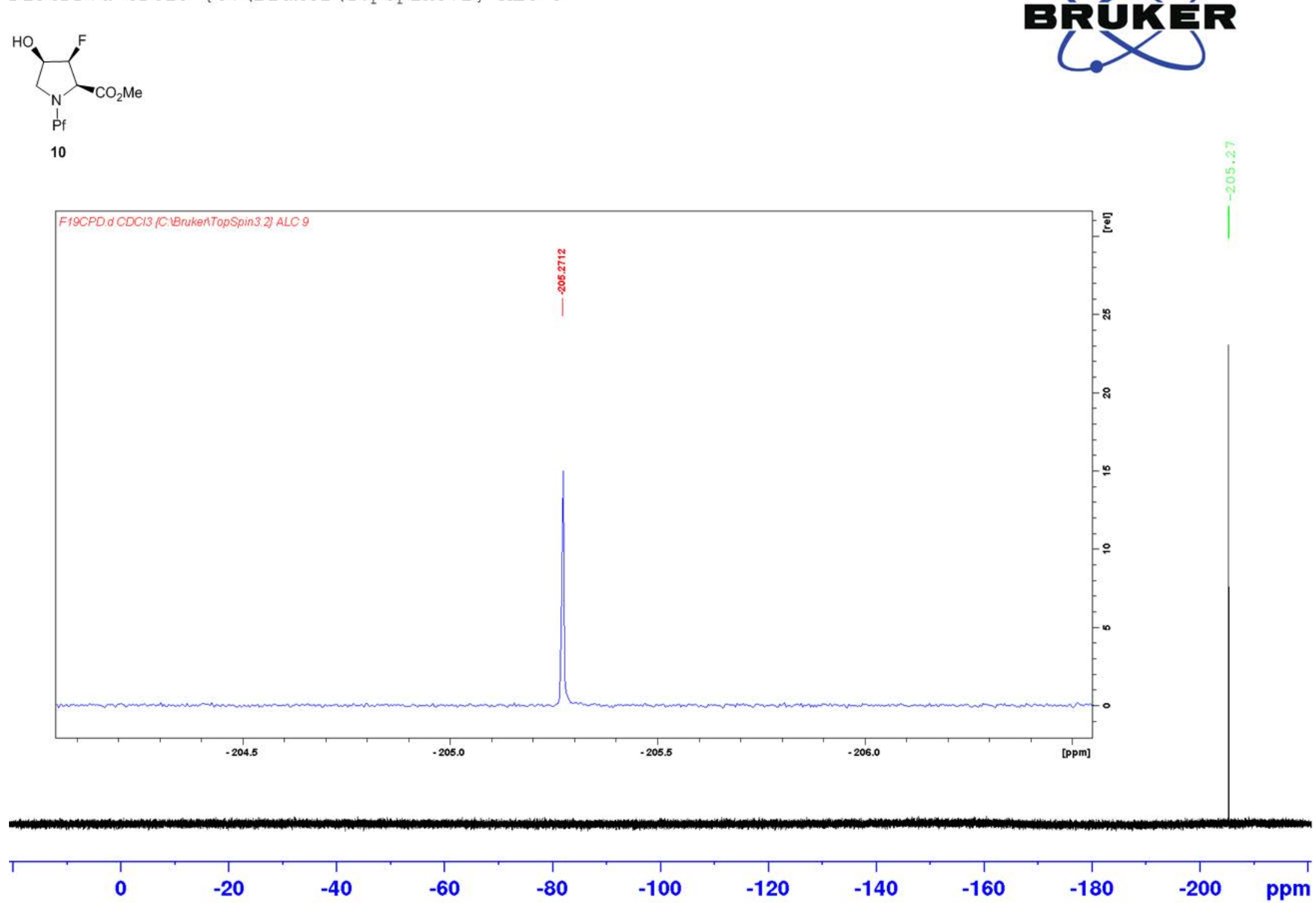


${ }^{13} \mathrm{C}-\mathrm{NMR}, \mathrm{CDCl}_{3}$
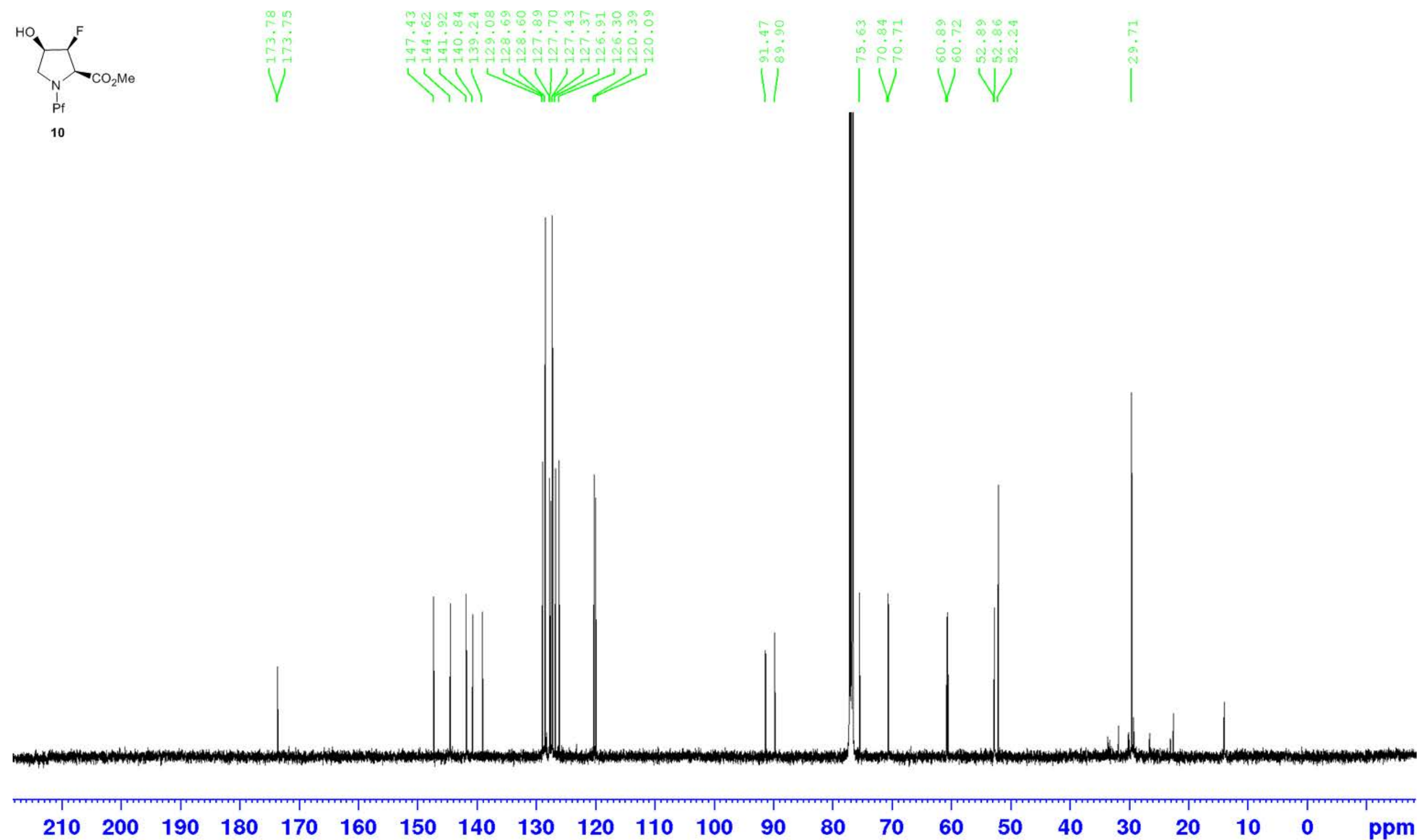
${ }^{1} \mathrm{H}-\mathrm{NMR}, \mathrm{CDCl}_{3}$

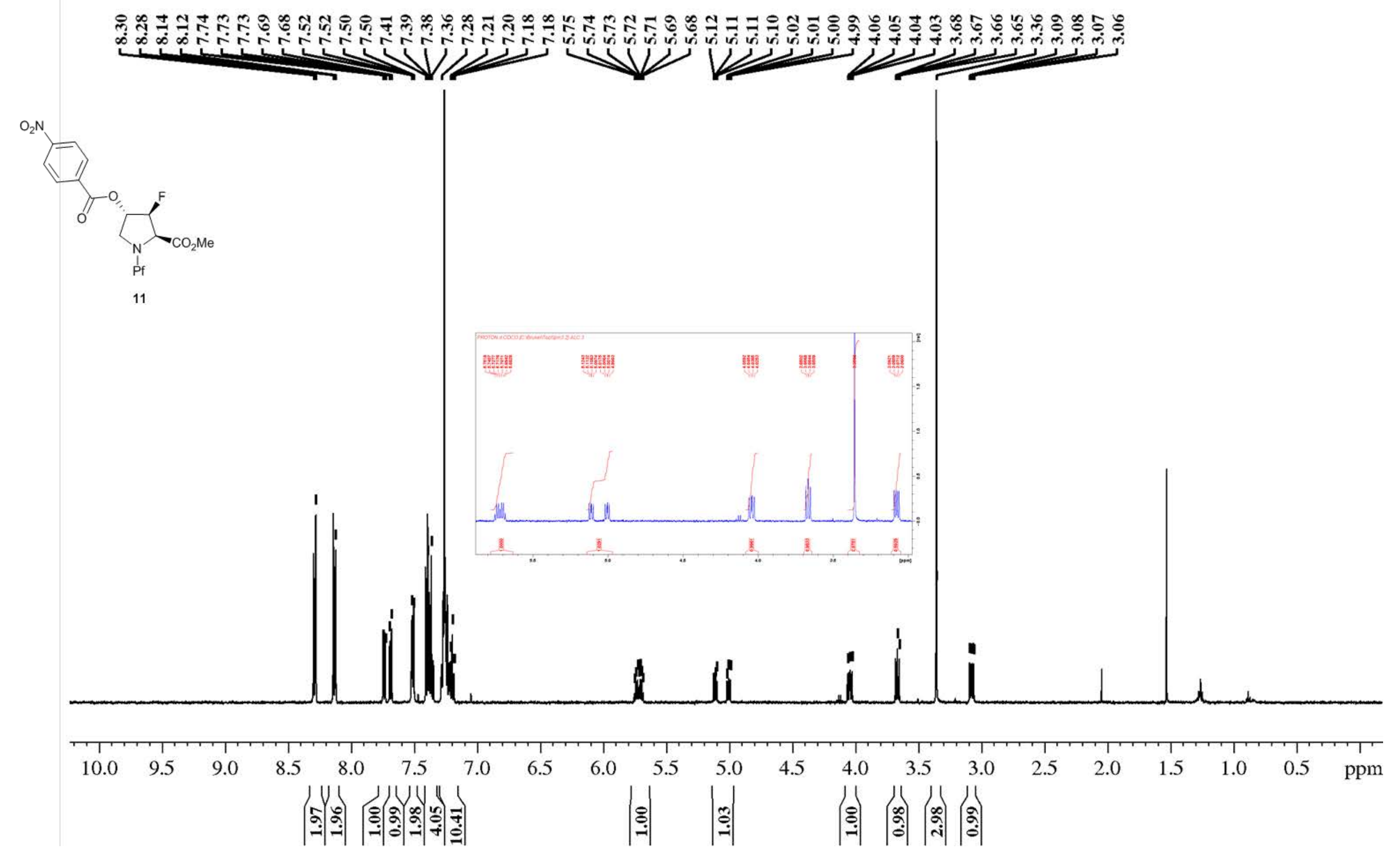


${ }^{19} \mathrm{~F}-\mathrm{NMR}, \mathrm{CDCl}_{3}$

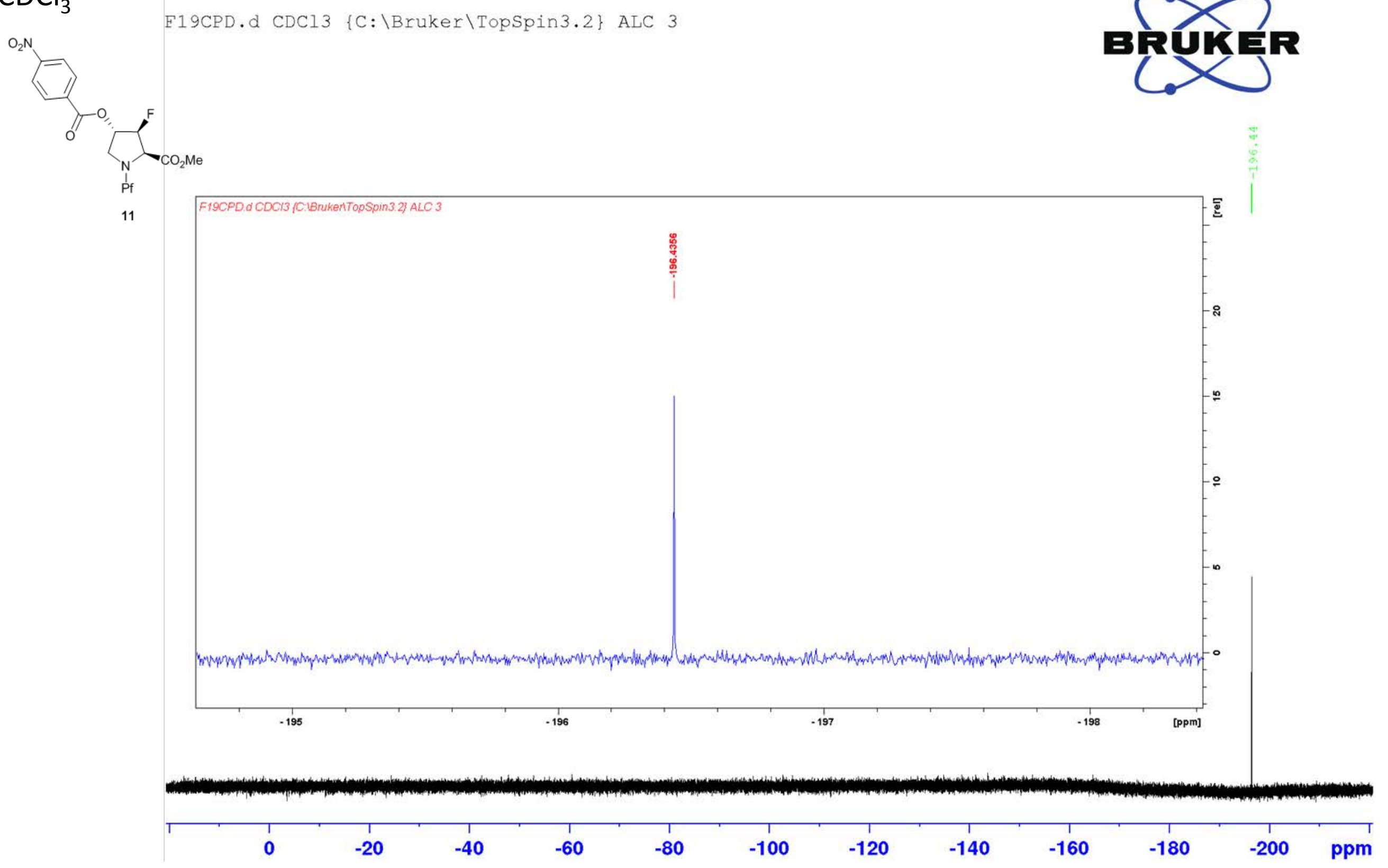


${ }^{13} \mathrm{C}-\mathrm{NMR}, \mathrm{CDCl}_{3}$

\section{BRUKER}

$\mathrm{O}_{2} \mathrm{~N}$

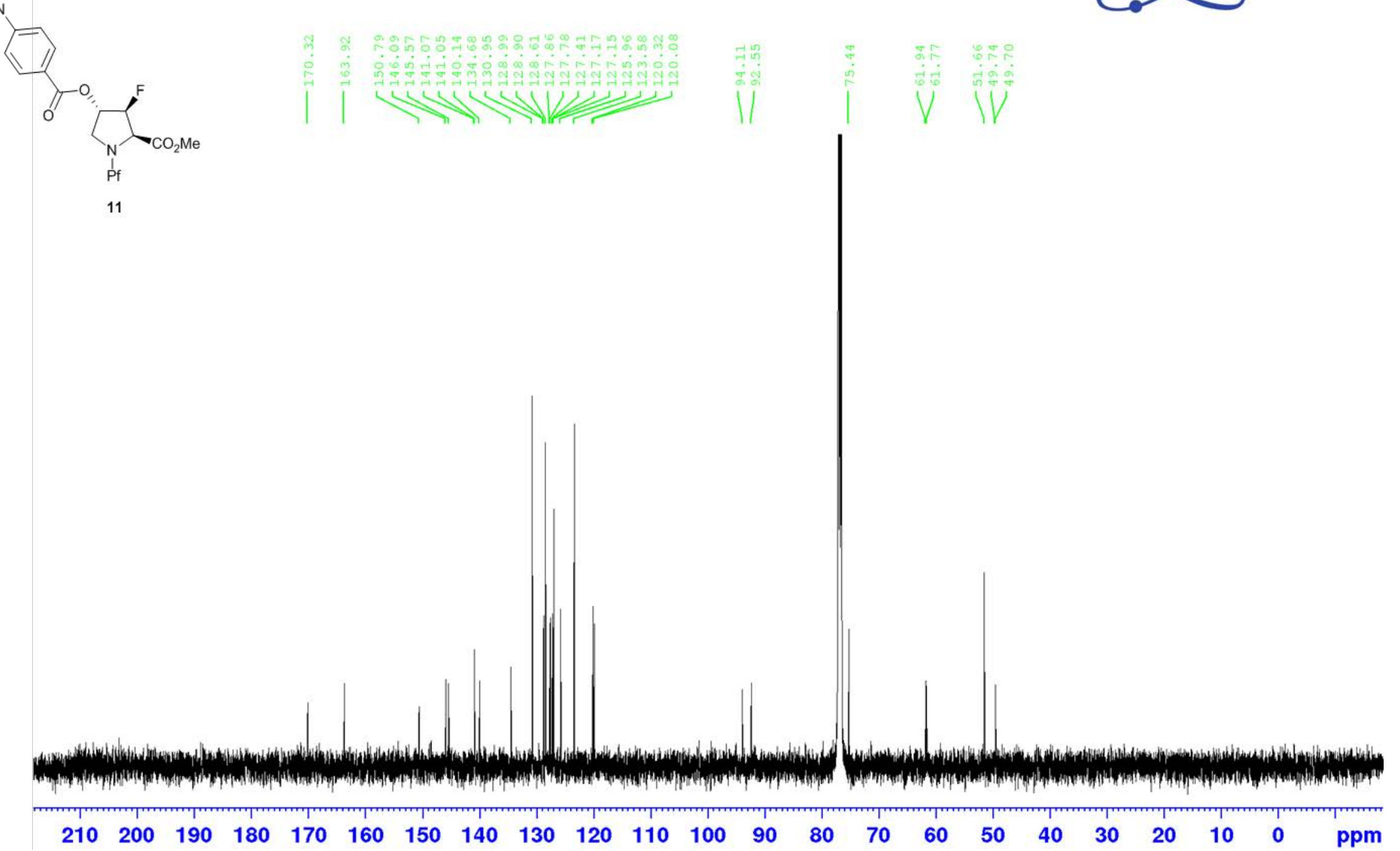


${ }^{1} \mathrm{H}-\mathrm{NMR}, \mathrm{D}_{2} \mathrm{O}$

PROTON.DAY D2O \{C: \Bruker \TopSpin3.2\} ALC 22

\section{PRURER}

$\sum_{\mathrm{N}}^{\mathrm{FO}}$

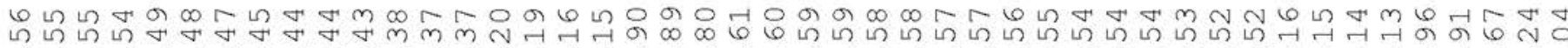
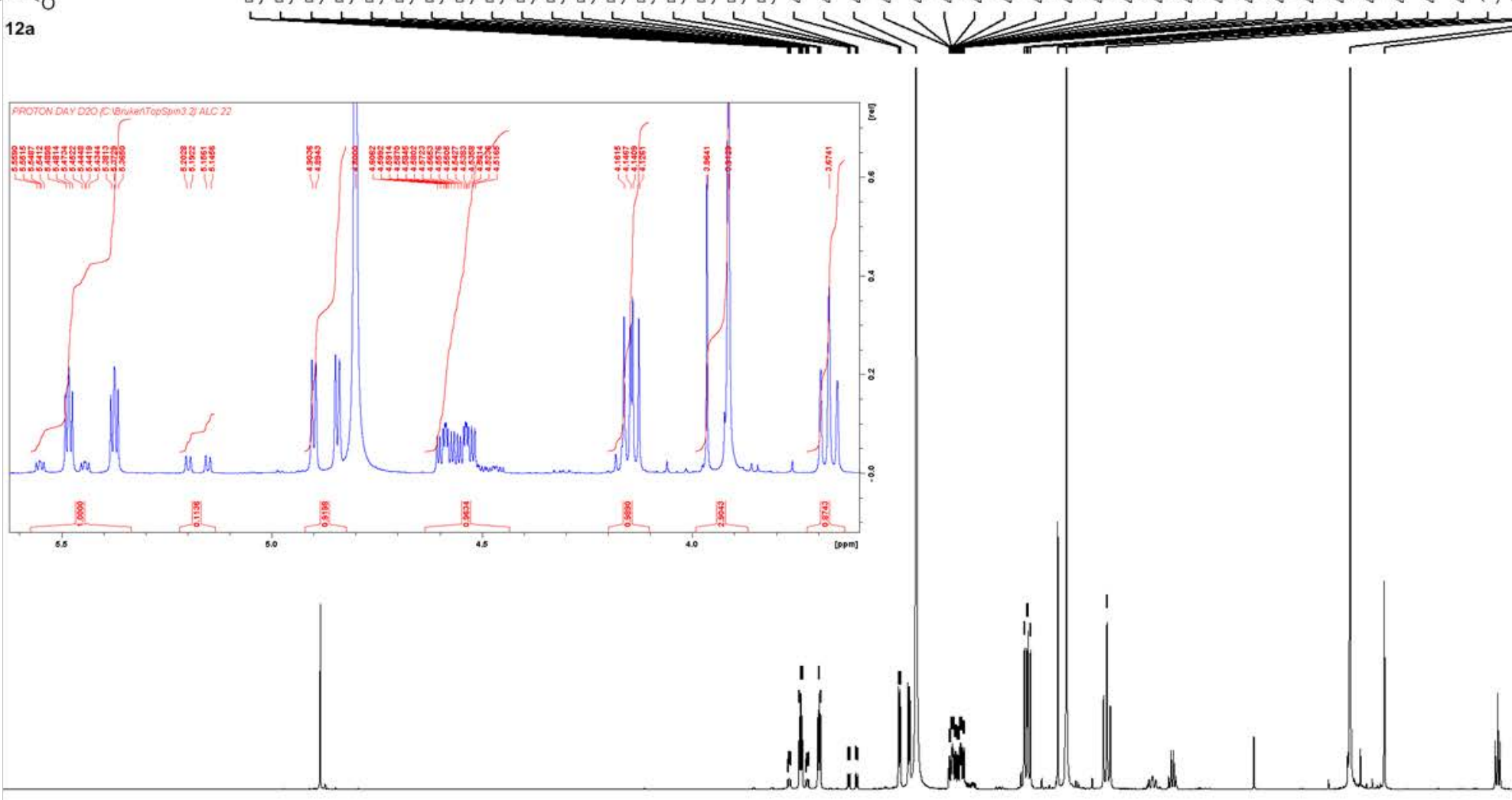

$10.0 \quad 9.5$
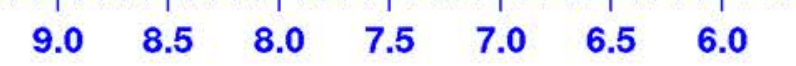

$\begin{array}{lllll}5.5 & 5.0 & 4.5 & 4.0 & 3.5\end{array}$

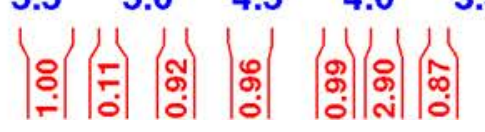

$\begin{array}{lllllll}3.0 & 2.5 & 2.0 & 1.5 & 1.0 & 0.5 & \mathrm{ppm}\end{array}$

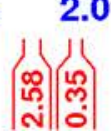


${ }^{19} \mathrm{~F}-\mathrm{NMR}, \mathrm{D}_{2} \mathrm{O}$

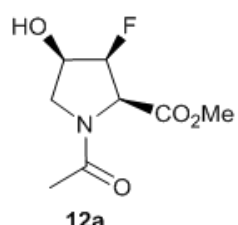

F19CPD.DAY D20 \{C: \Bruker \TopSpin3.2\} ALC 22
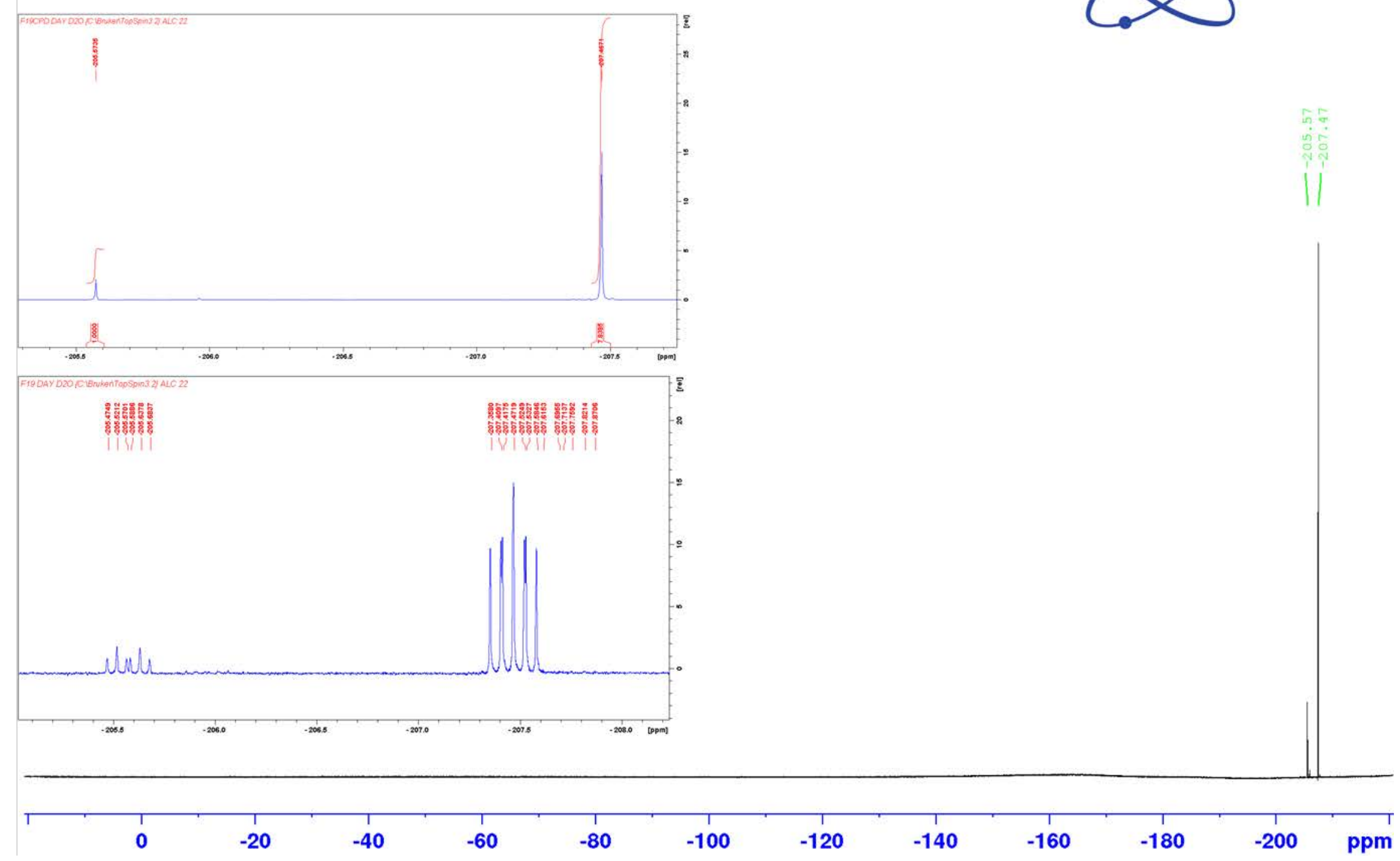

\section{apperen}

( $)$ 
${ }^{13} \mathrm{C}-\mathrm{NMR}, \mathrm{D}_{2} \mathrm{O}$

BRUKER
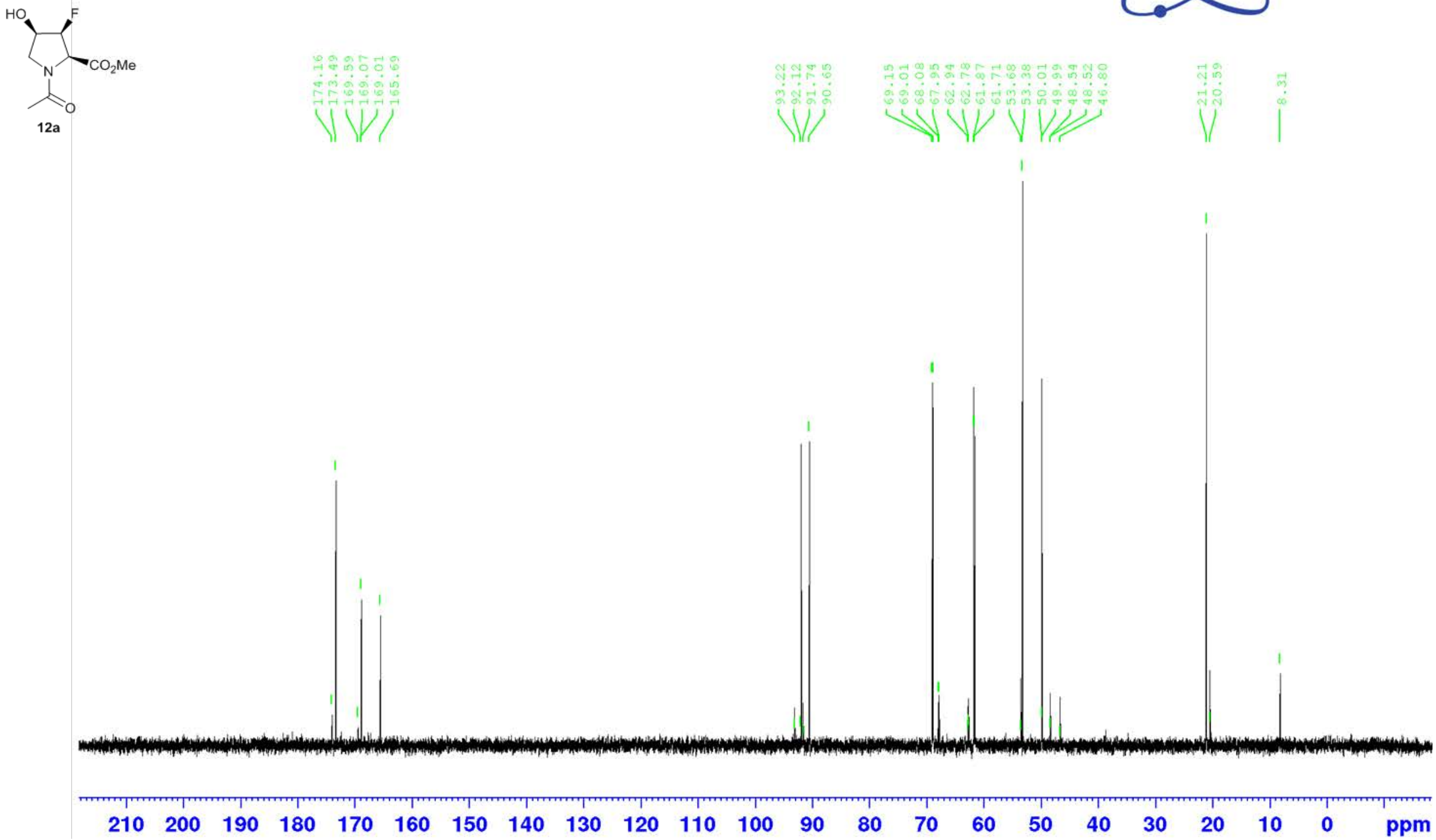
${ }^{1} \mathrm{H}-\mathrm{NMR}, \mathrm{D}_{2} \mathrm{O}$

PROTON.NIGHT D2O \{C: \Bruker \TOpSpin3.2\} ALC 23
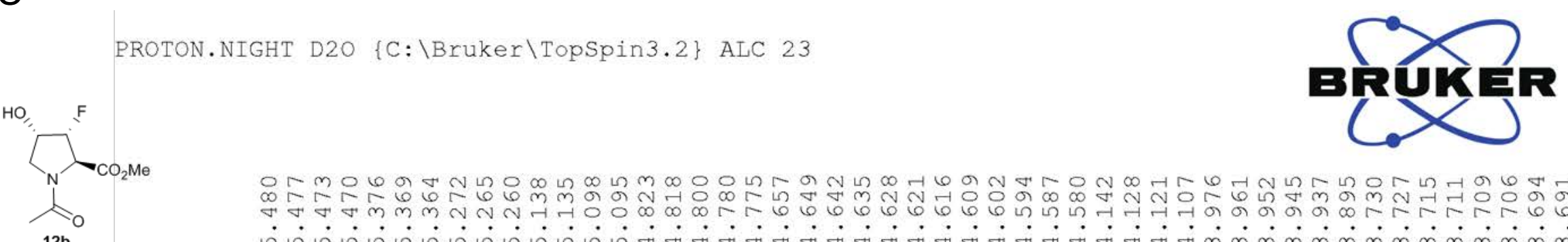

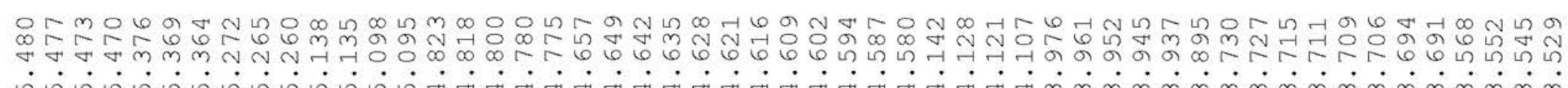

12b
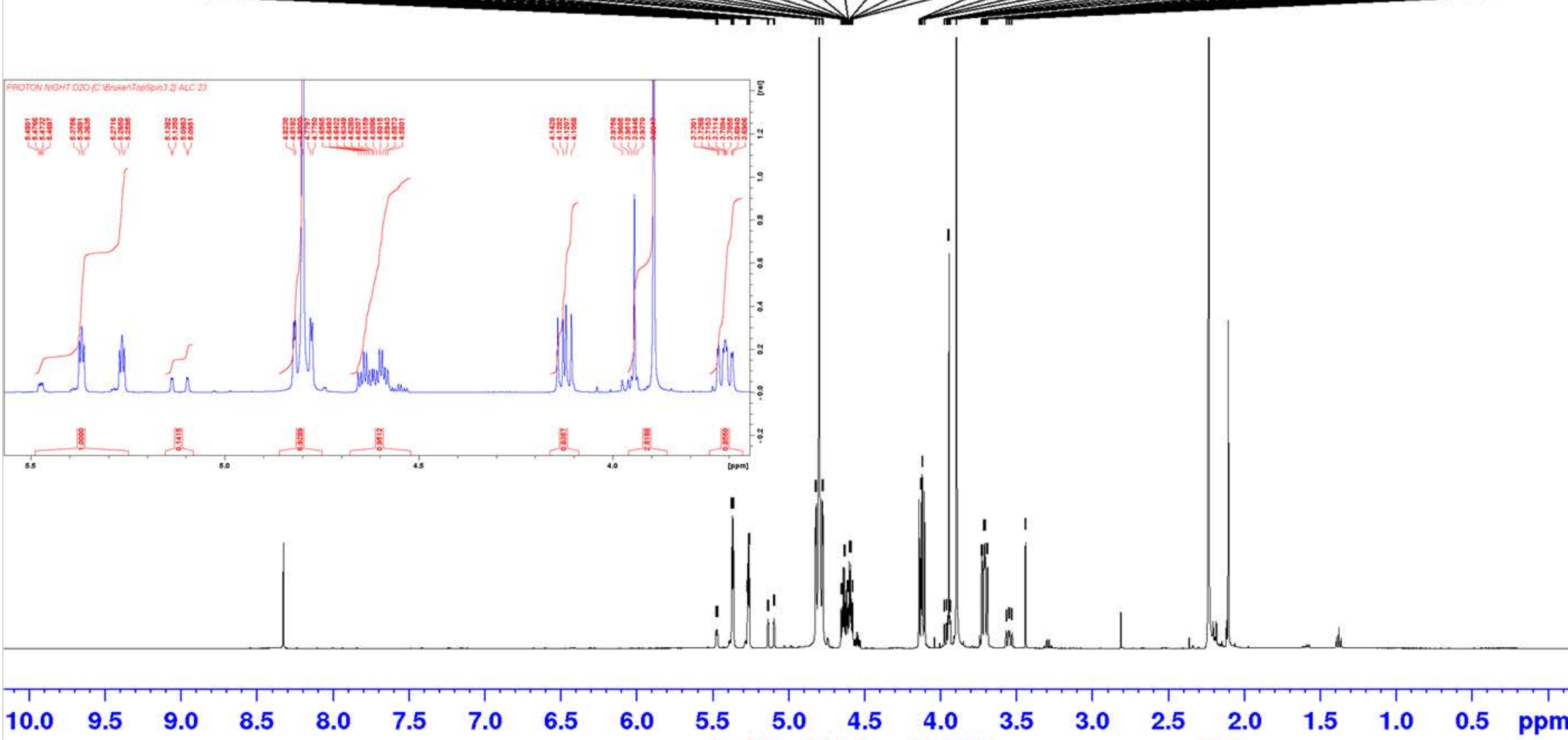

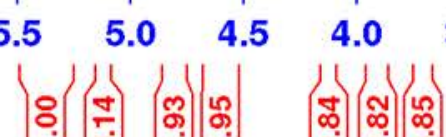

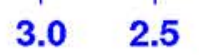

$\begin{array}{lllll}2.0 & 1.5 & 1.0 & 0.5 & \mathrm{ppm}\end{array}$ | 
${ }^{19} \mathrm{~F}-\mathrm{NMR}, \mathrm{D}_{2} \mathrm{O}$

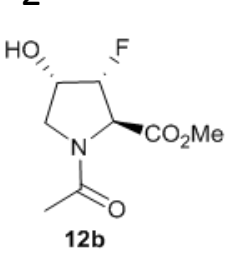

F19CPD.DAY D20 \{C: \Bruker \Topspin3.2\} ALC 23
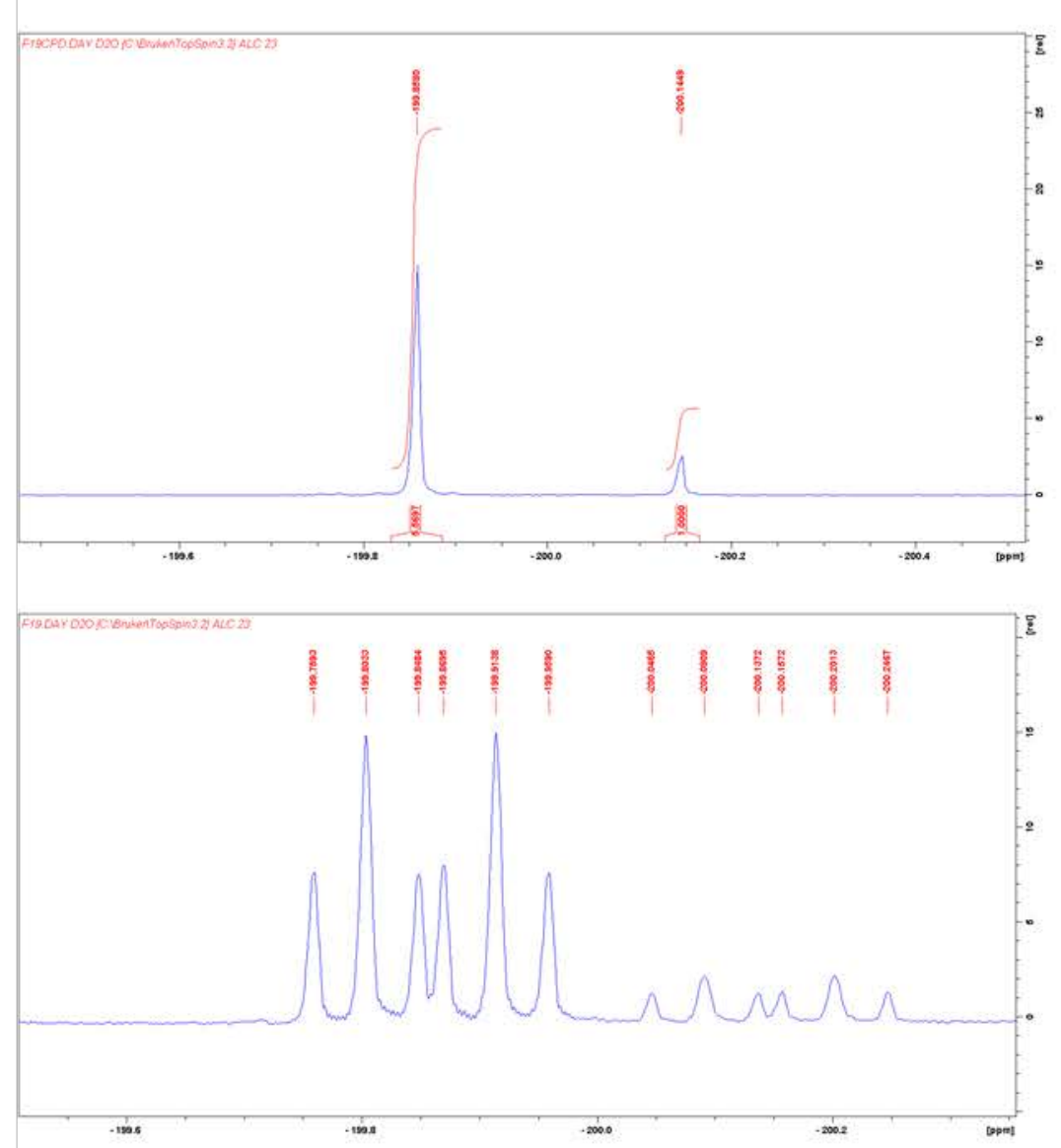

arumer

v

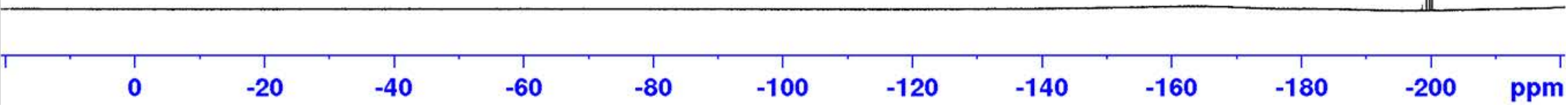




\section{${ }^{13} \mathrm{C}-\mathrm{NMR}, \mathrm{D}_{2} \mathrm{O}$}

\section{BR⿴囗十) \\ C.}



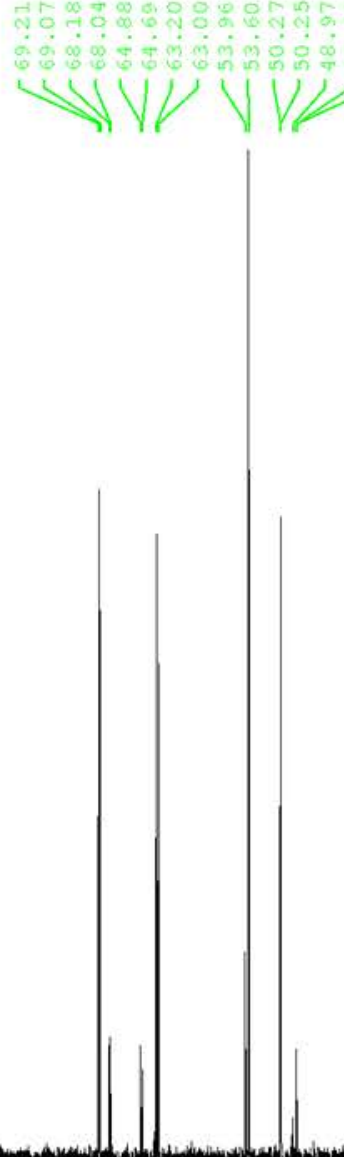

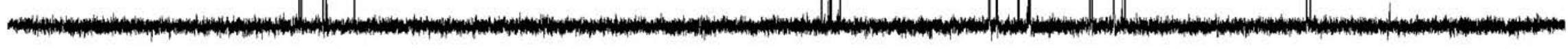




\section{efrêren}

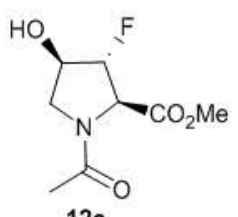

$(>)$

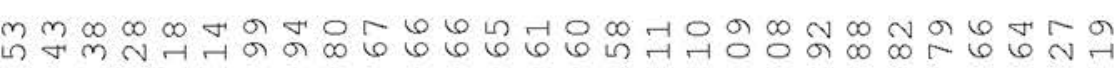
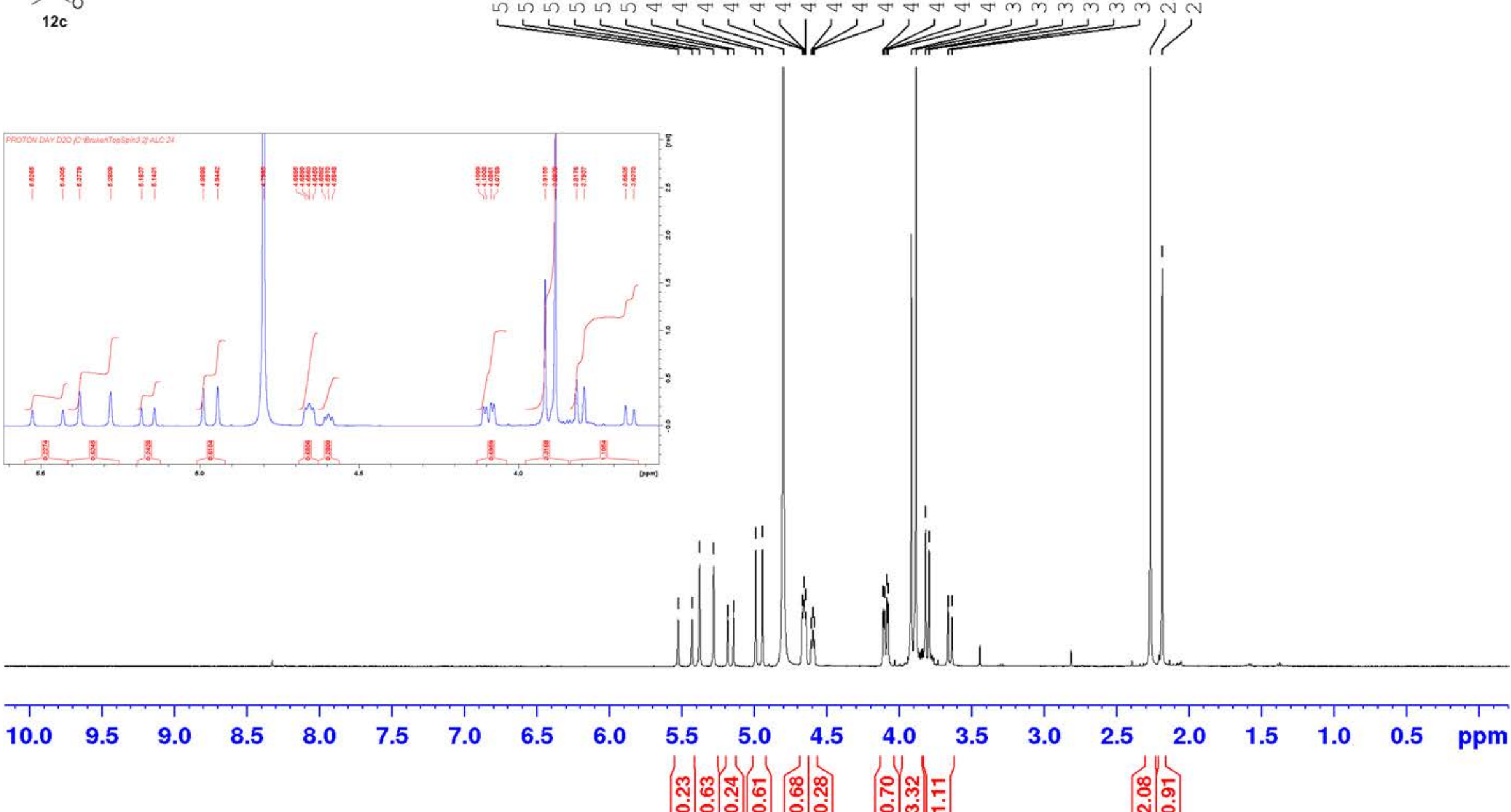
${ }^{19} \mathrm{~F}-\mathrm{NMR}, \mathrm{D}_{2} \mathrm{O}$

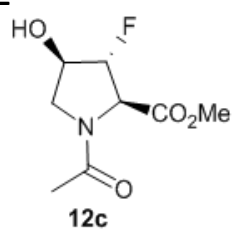

F19CPD.DAY D20 \{C: \Bruker \TopSpin3.2\} ALC 24

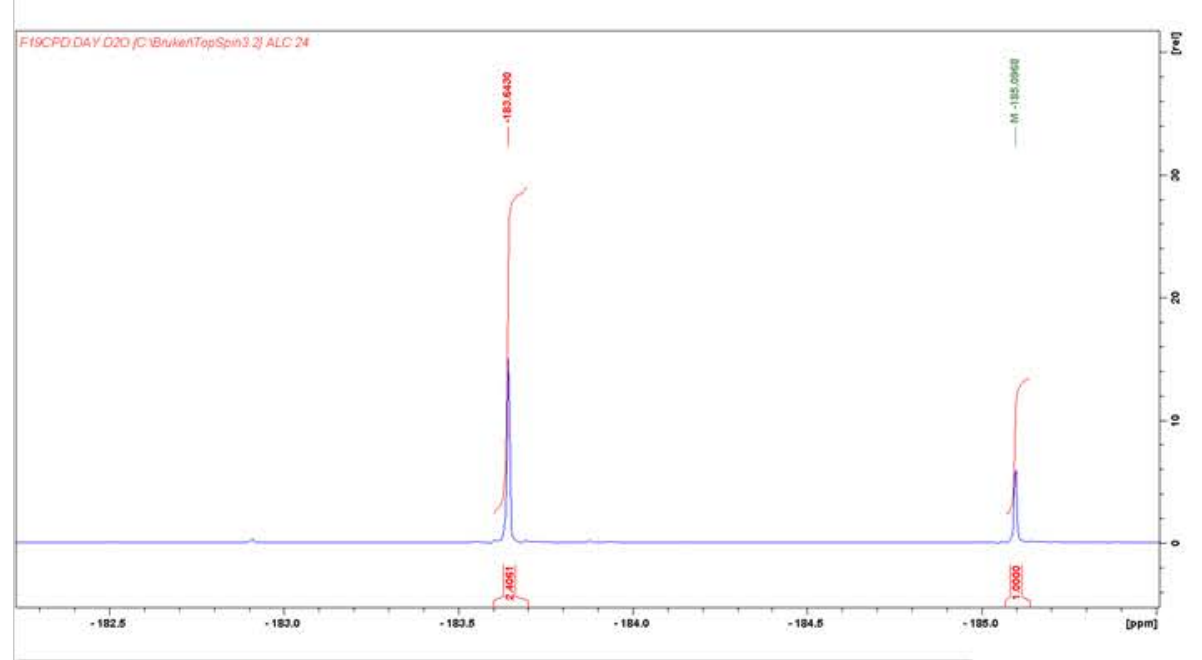

PRUKER
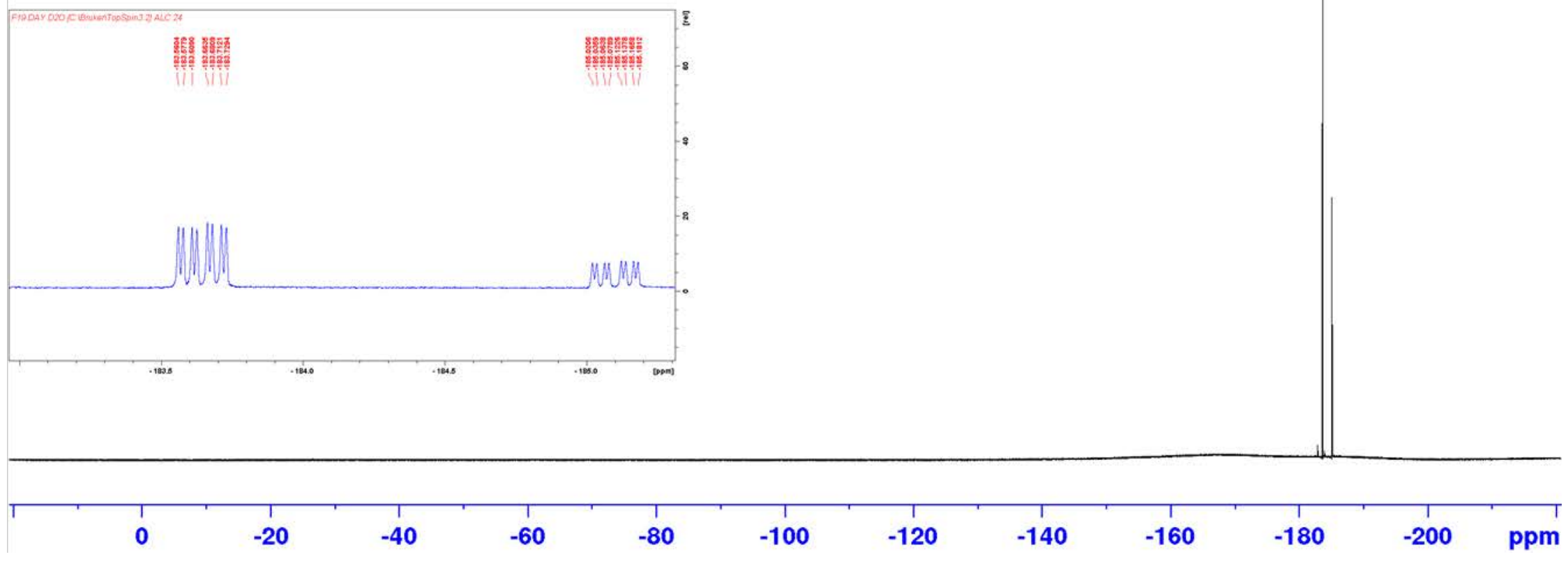
${ }^{13} \mathrm{C}-\mathrm{NMR}, \mathrm{D}_{2} \mathrm{O}$

\section{BR UXER}
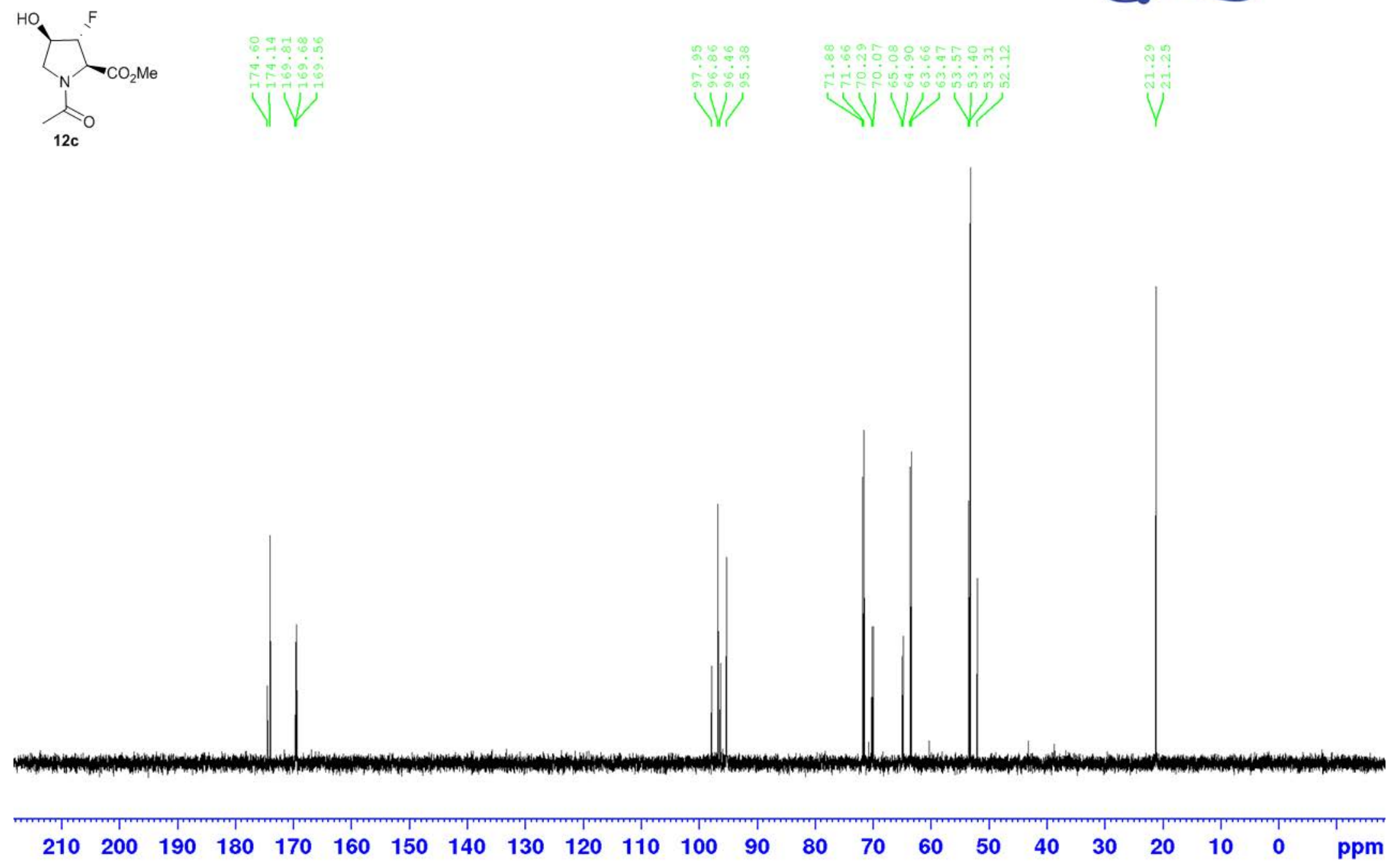
${ }^{1} \mathrm{H}-\mathrm{NMR}, \mathrm{D}_{2} \mathrm{O}$

PROTON.DAY D2O \{C: \Bruker TTopSpin3.2\} ALC 25

\section{BRUKER}

(12d

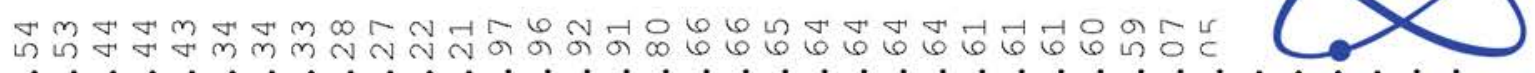

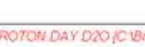

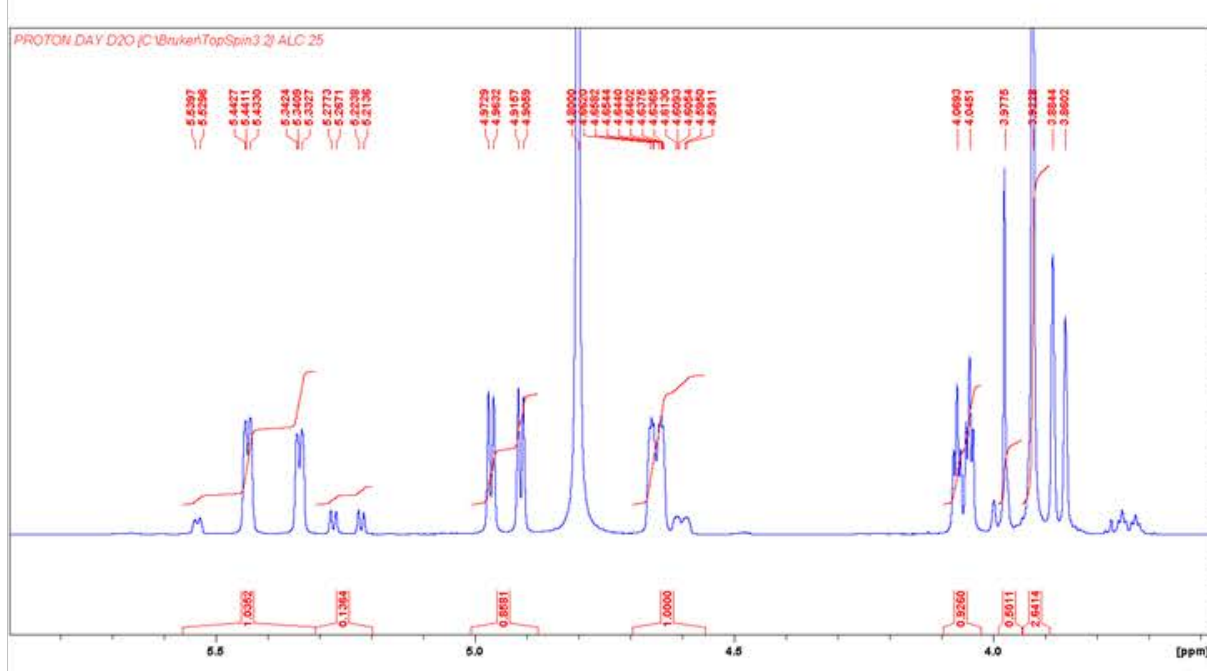

i $\left|\begin{array}{ll}1 & 11 \\ 1 & 11 \\ 11 & 11\end{array}\right| \mid$
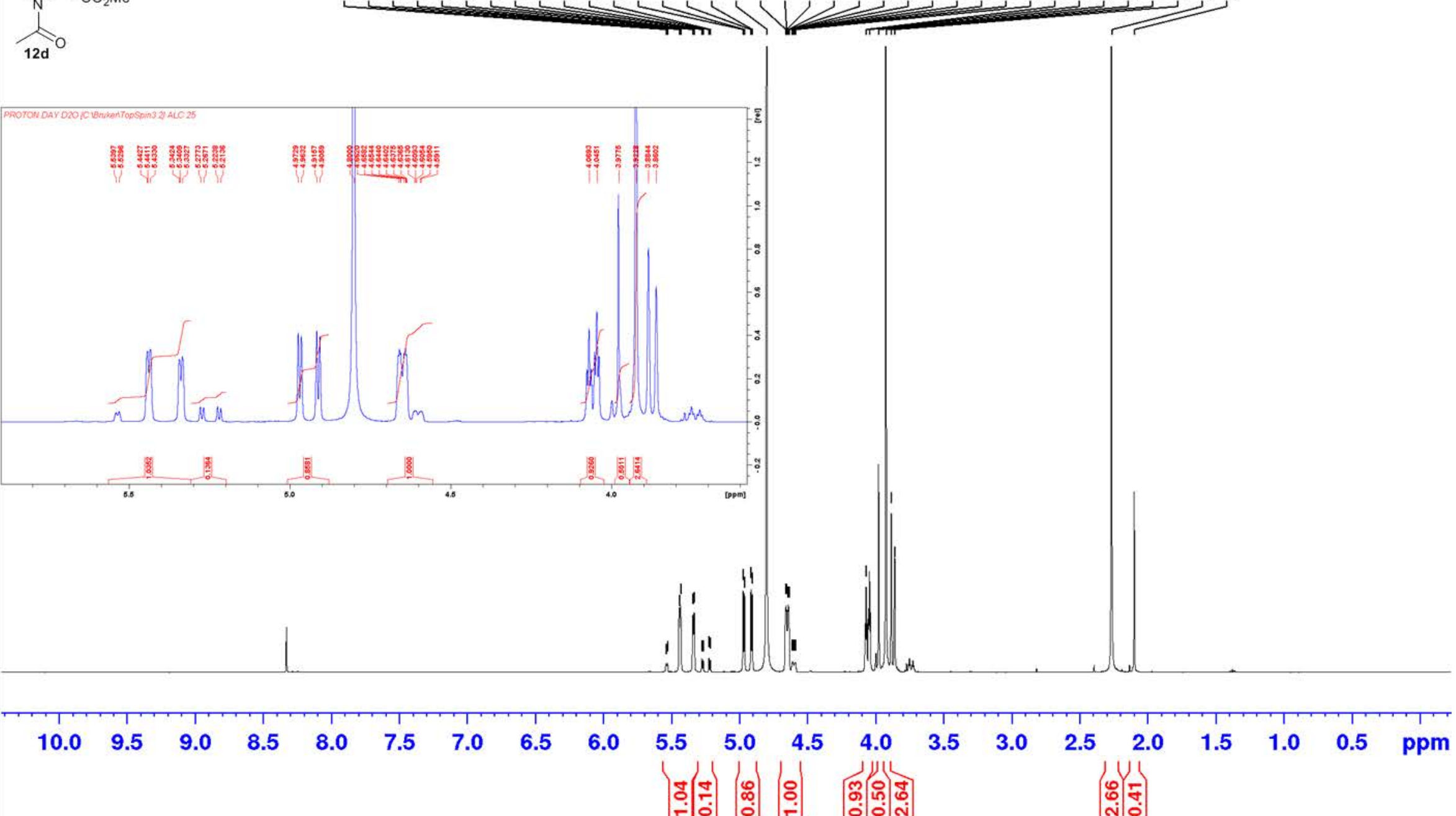

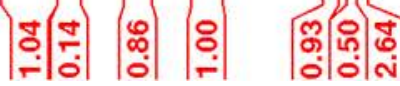


${ }^{19}$ F-NMR, $\mathrm{D}_{2} \mathrm{O}$

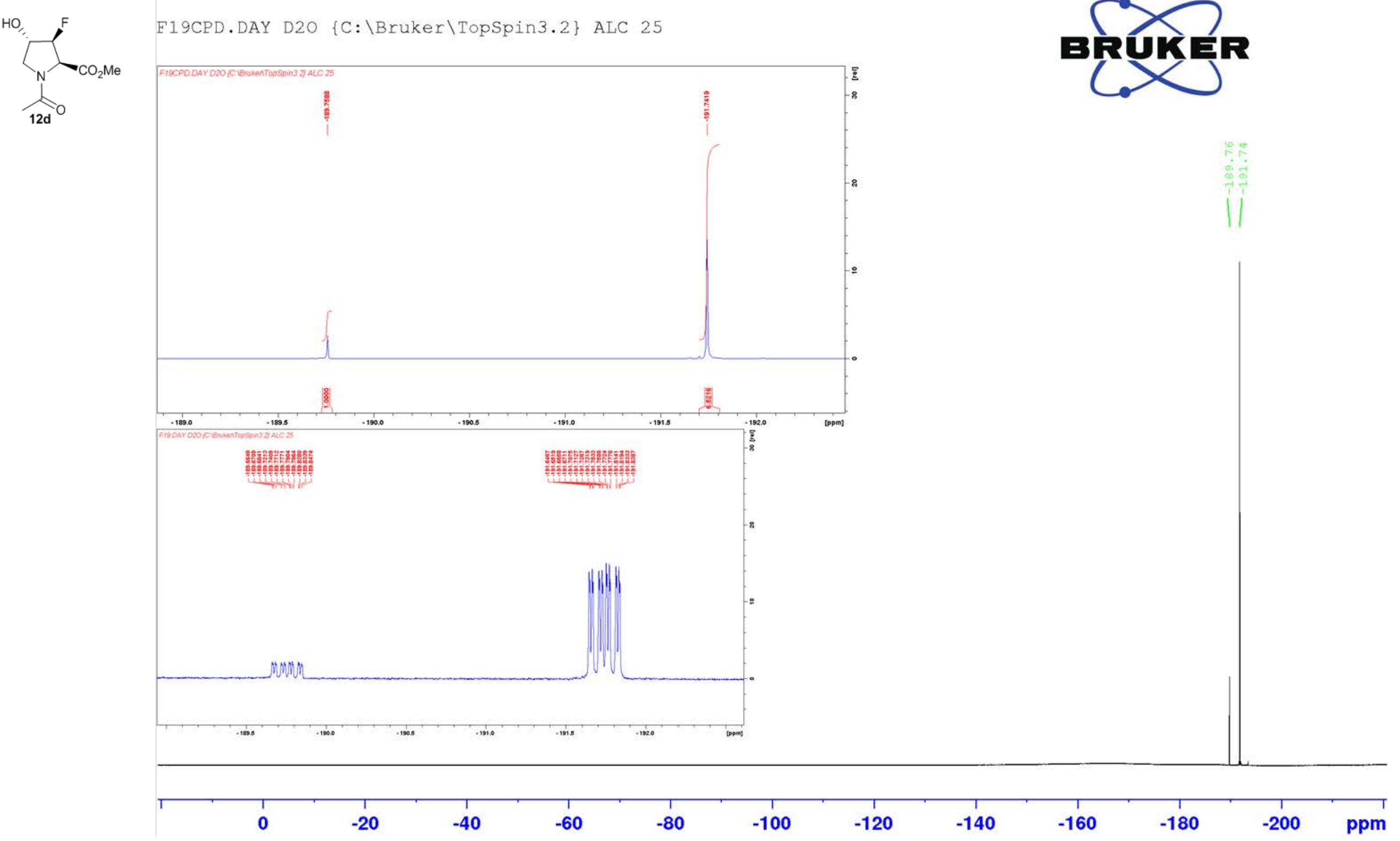


${ }^{13} \mathrm{C}-\mathrm{NMR}, \mathrm{D}_{2} \mathrm{O}$

HO

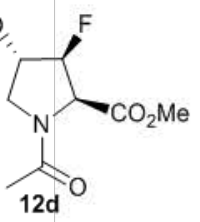

CRUKER

${ }_{12 \mathrm{~d}} \mathrm{O}$

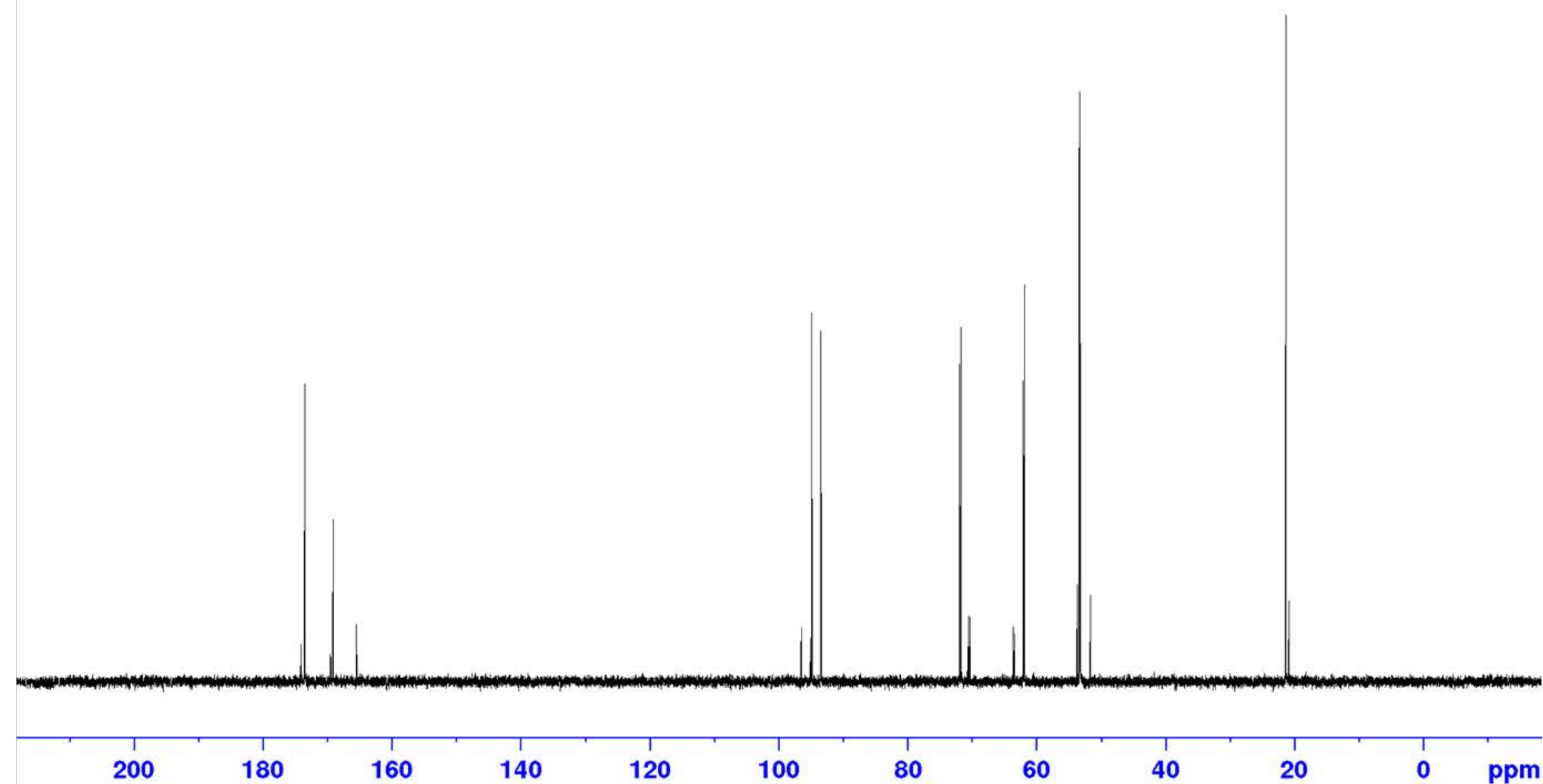


${ }^{1} \mathrm{H}-\mathrm{NMR}, \mathrm{CD}_{3} \mathrm{OD}$

$\mathrm{OH}$

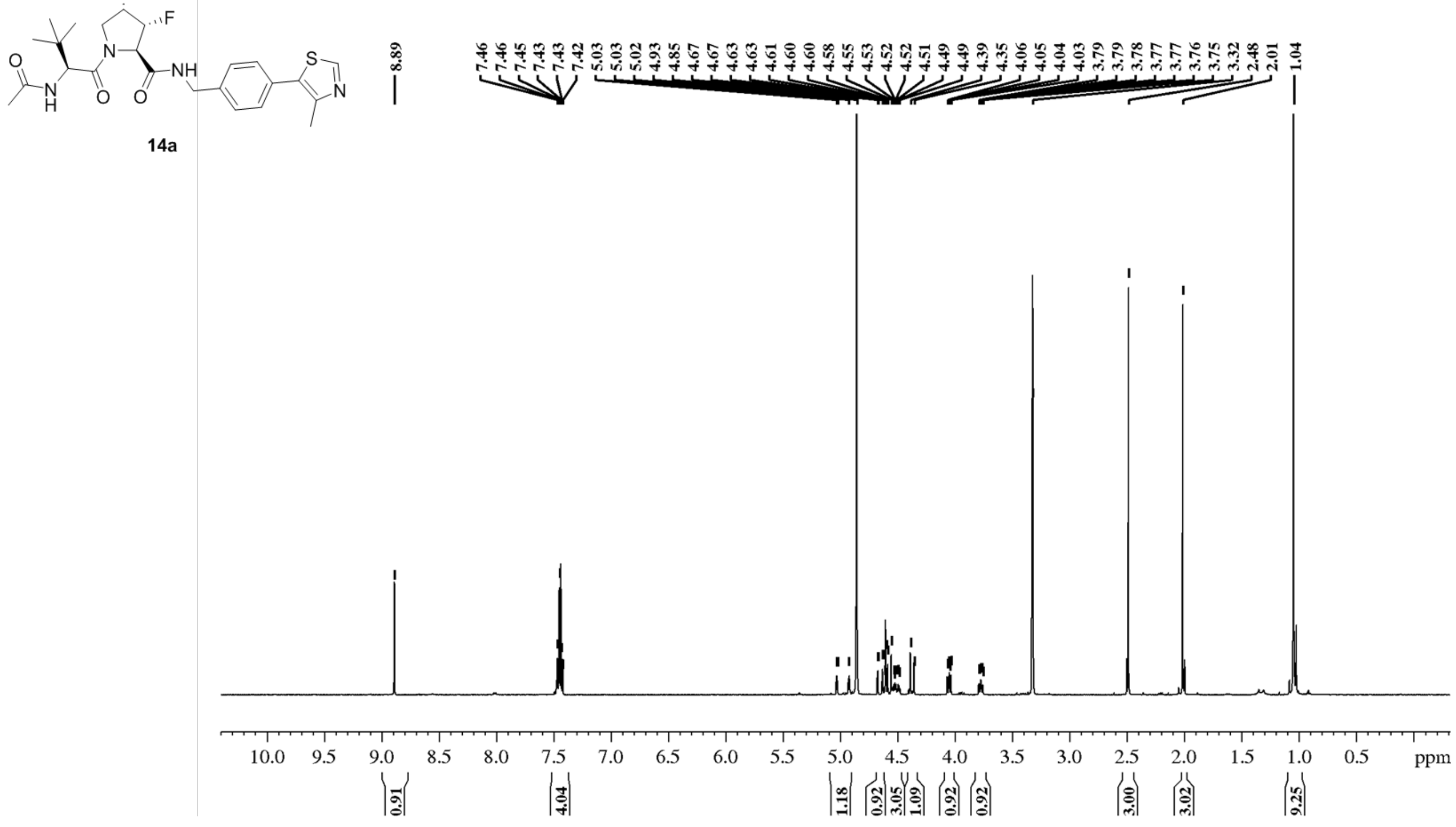


${ }^{19} \mathrm{~F}-\mathrm{NMR}, \mathrm{CD}_{3} \mathrm{OD}$

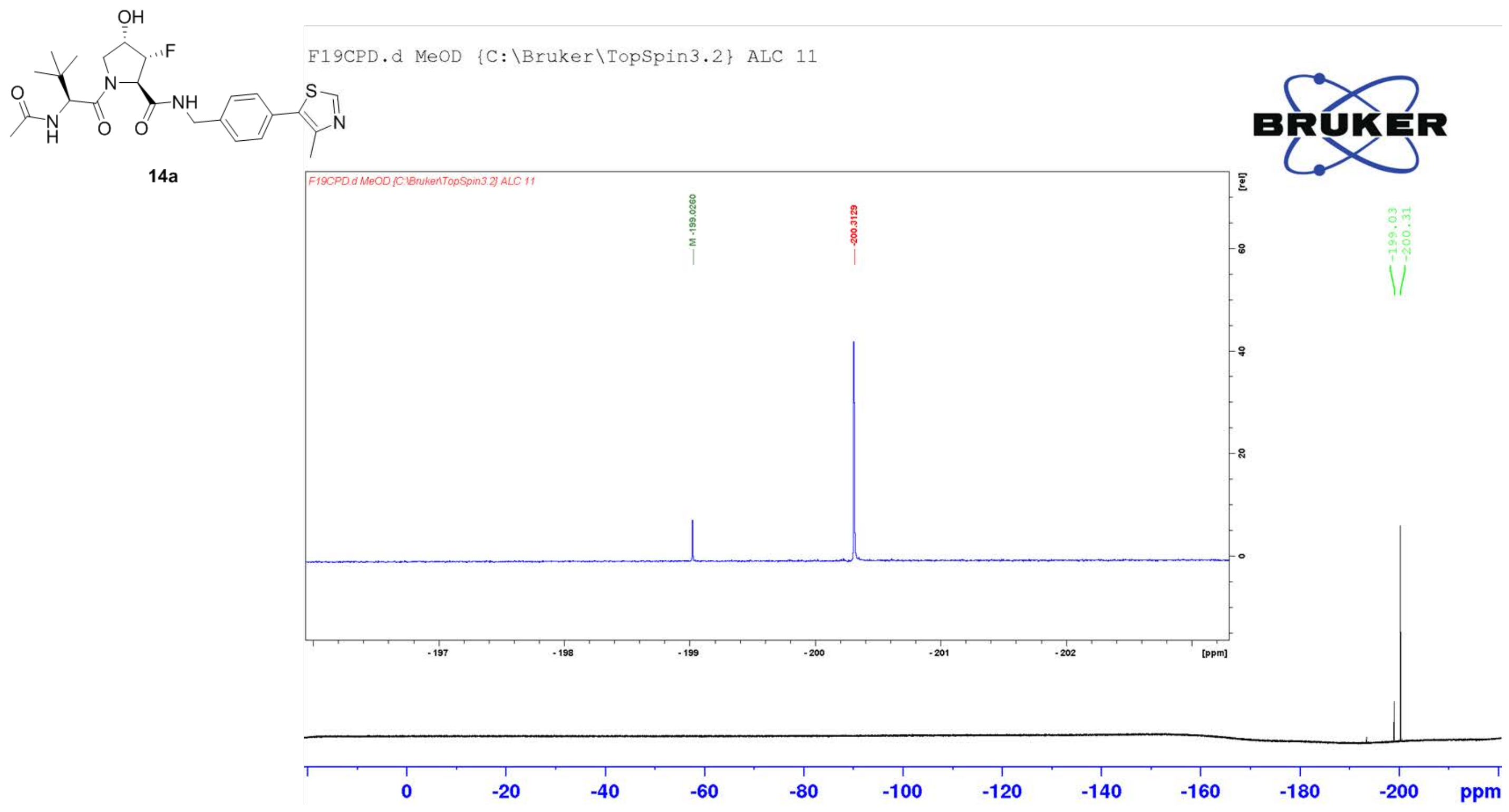


${ }^{13} \mathrm{C}-\mathrm{NMR}, \mathrm{CD}_{3} \mathrm{OD}$
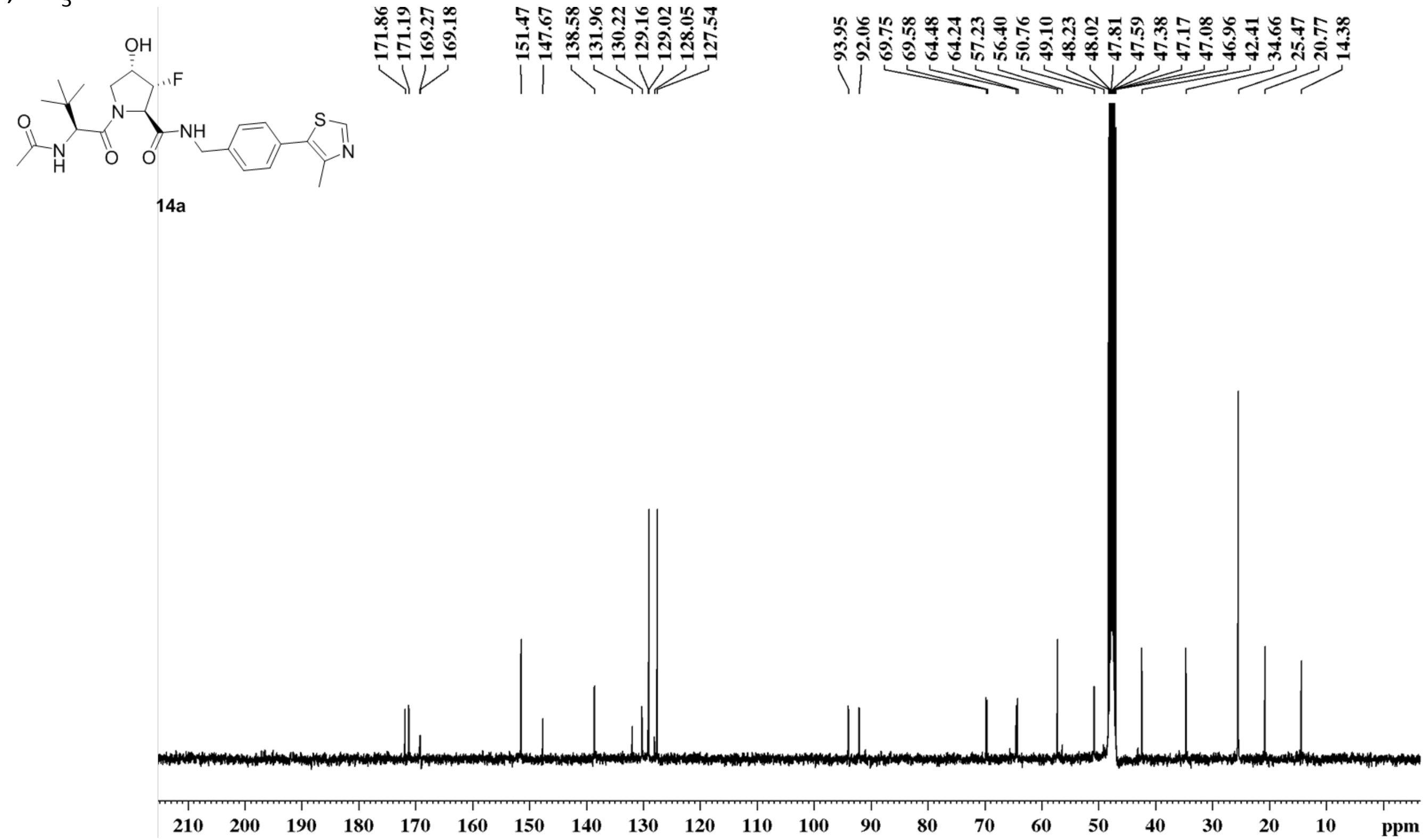
${ }^{1} \mathrm{H}-\mathrm{NMR}, \mathrm{CD}_{3} \mathrm{OD}$

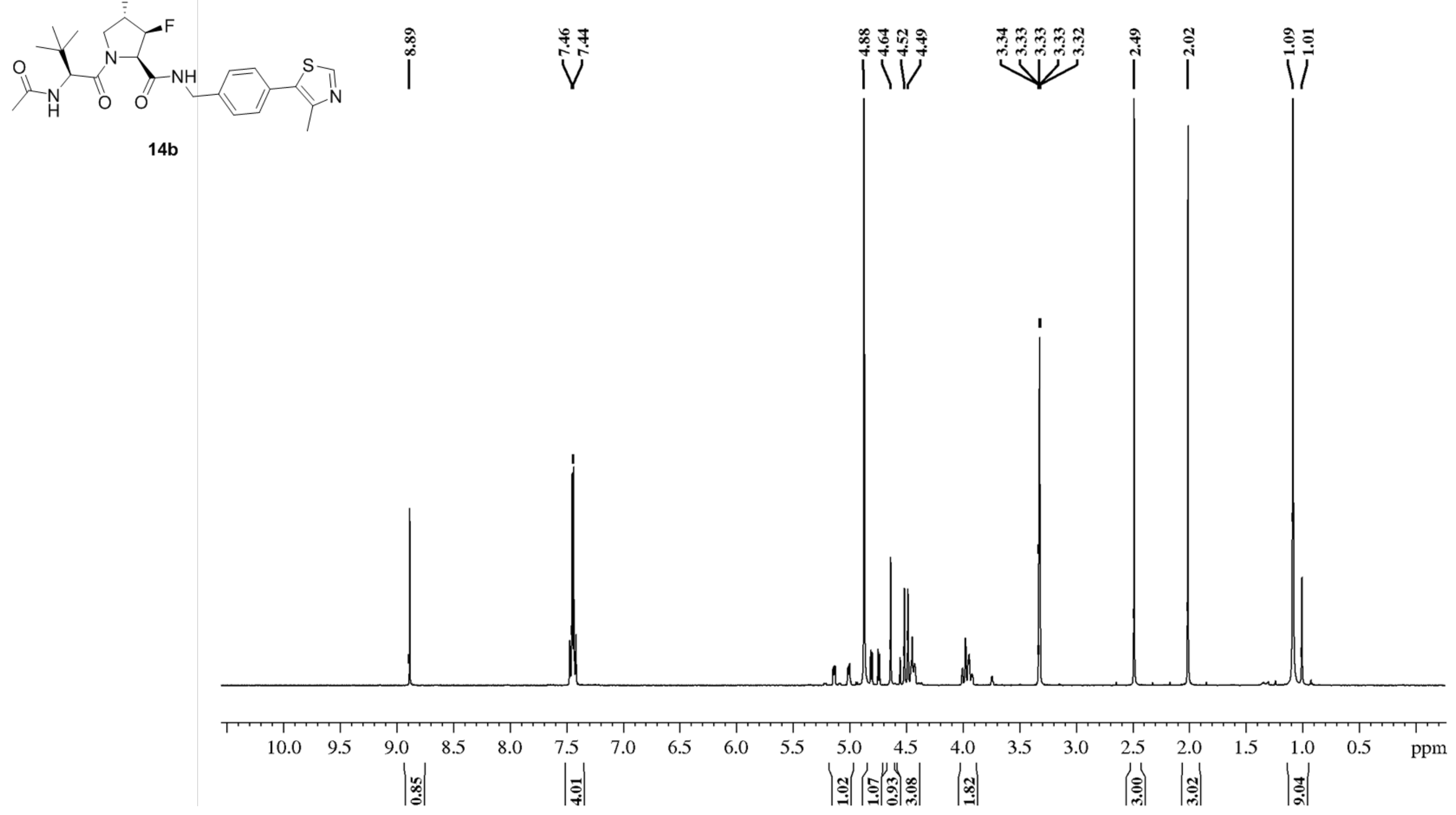


${ }^{19} \mathrm{~F}-\mathrm{NMR}, \mathrm{CD}_{3} \mathrm{OD}$

(14b

F19CPD.d MeOD \{C: \Bruker \TopSpin3.2\} ALC 6

BRuker N

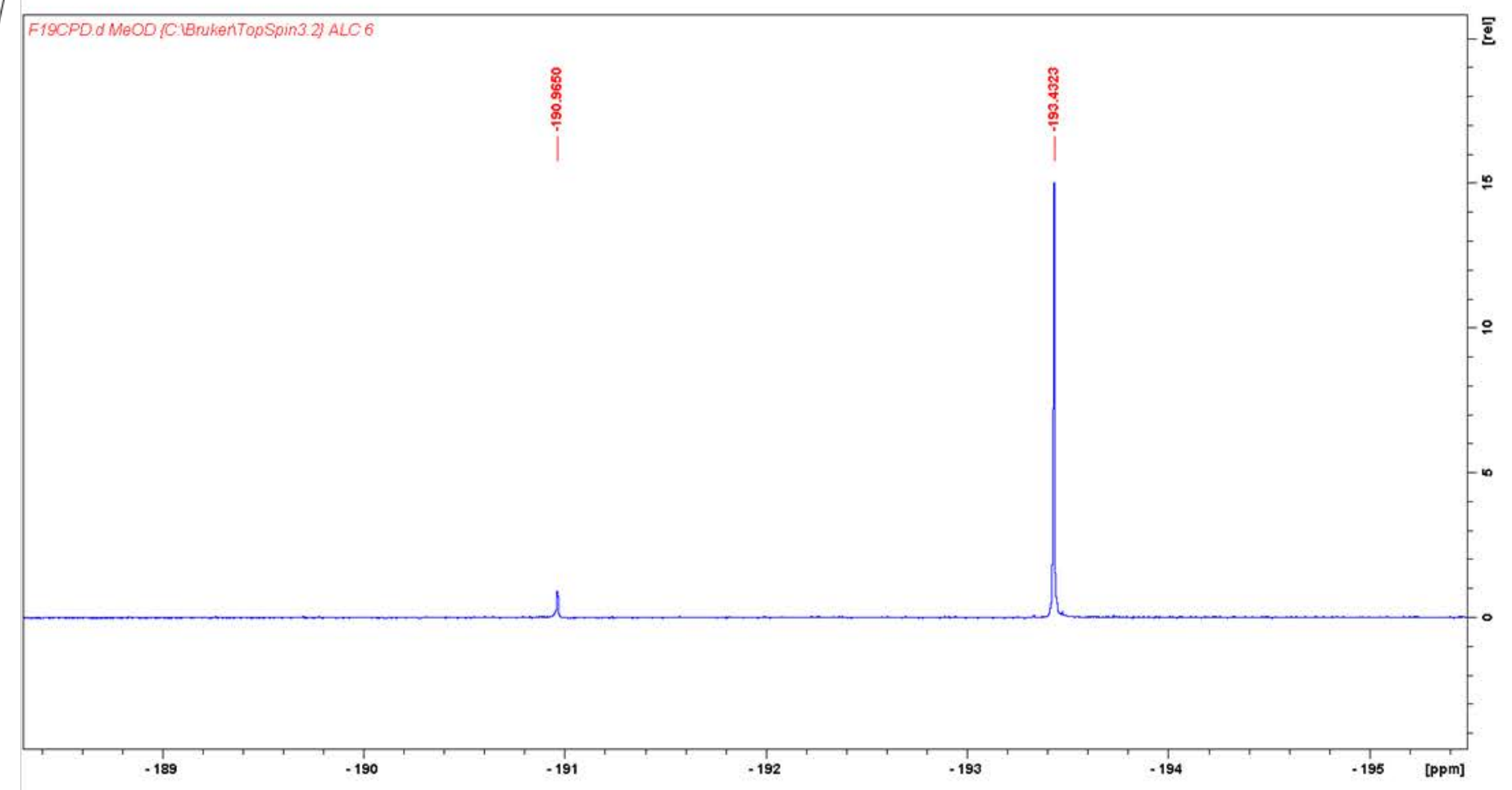

$C$

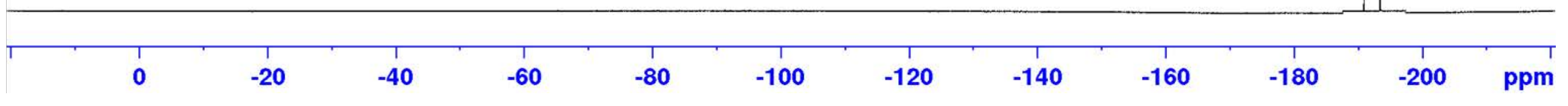


${ }^{13} \mathrm{C}-\mathrm{NMR}, \mathrm{CD}_{3} \mathrm{OD}$

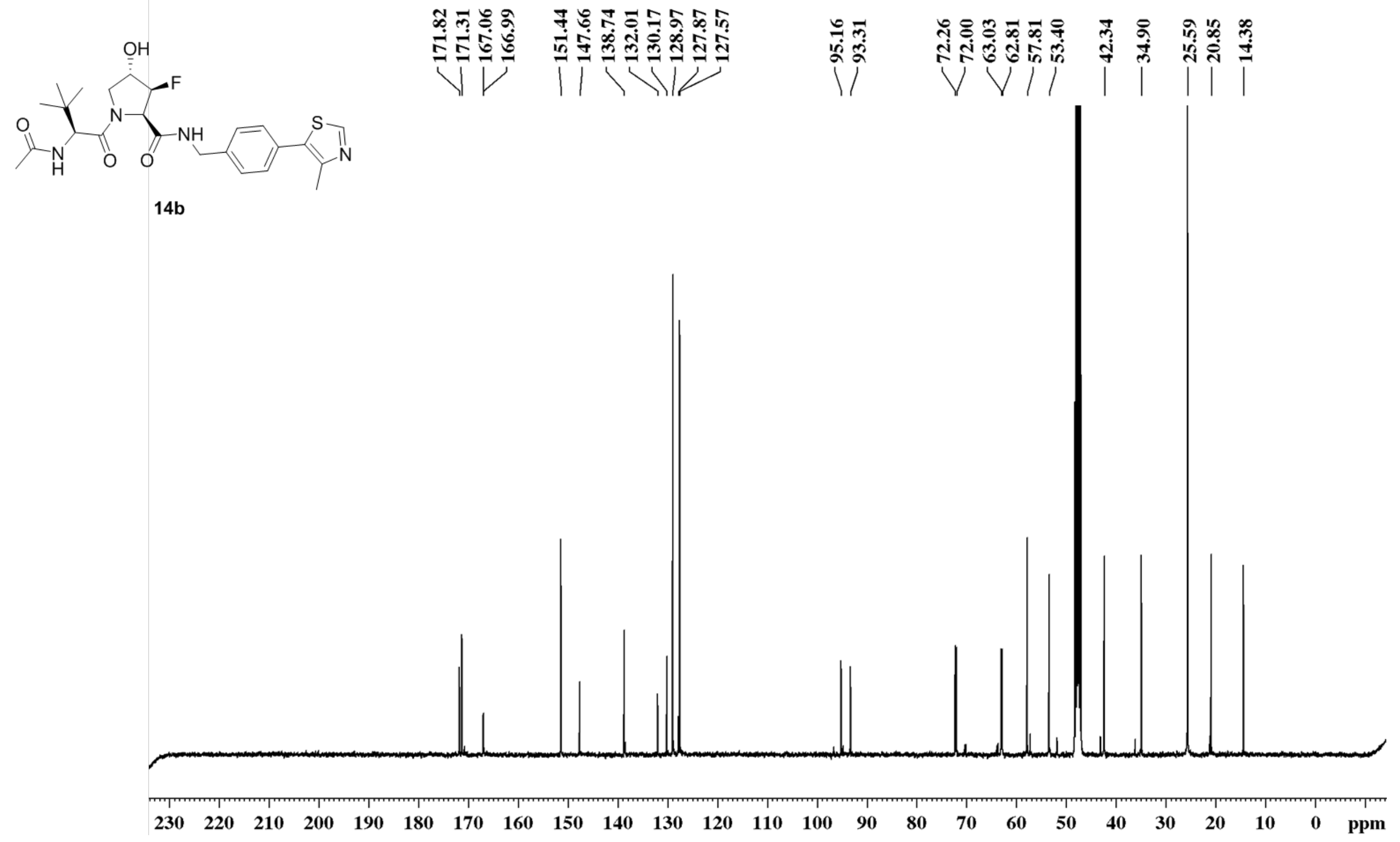




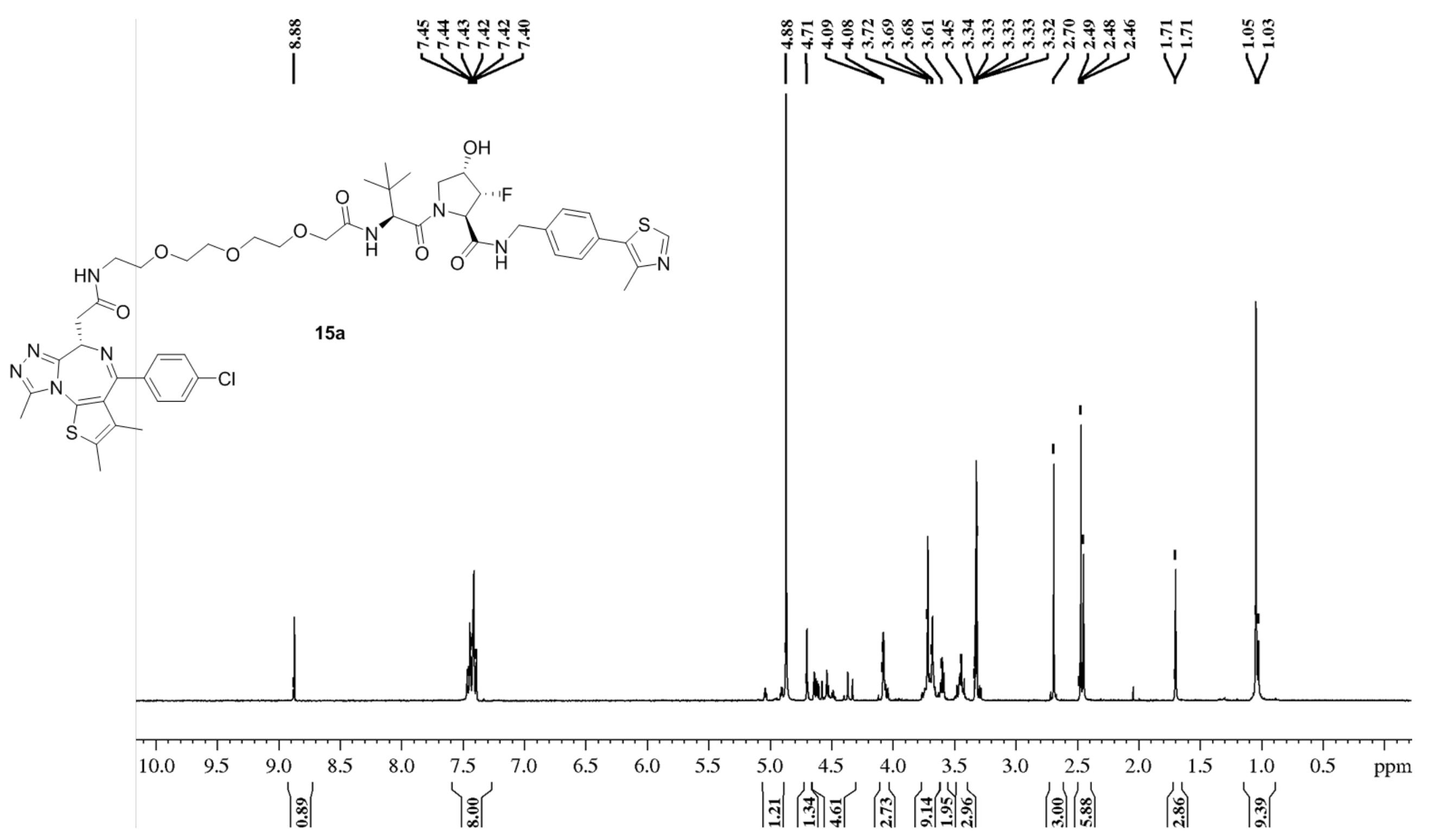


${ }^{19} \mathrm{~F}-\mathrm{NMR}, \mathrm{CD}_{3} \mathrm{OD}$

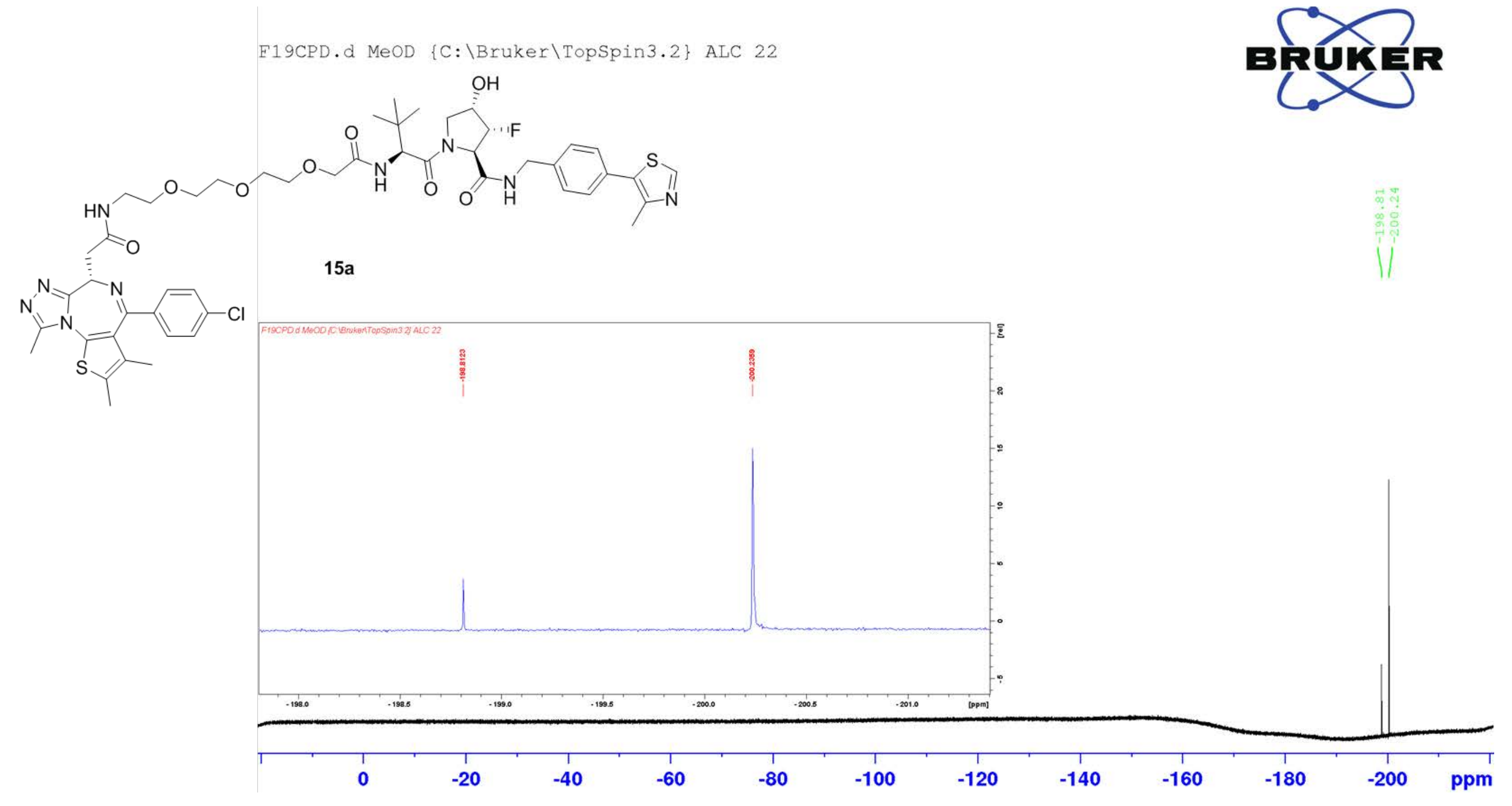




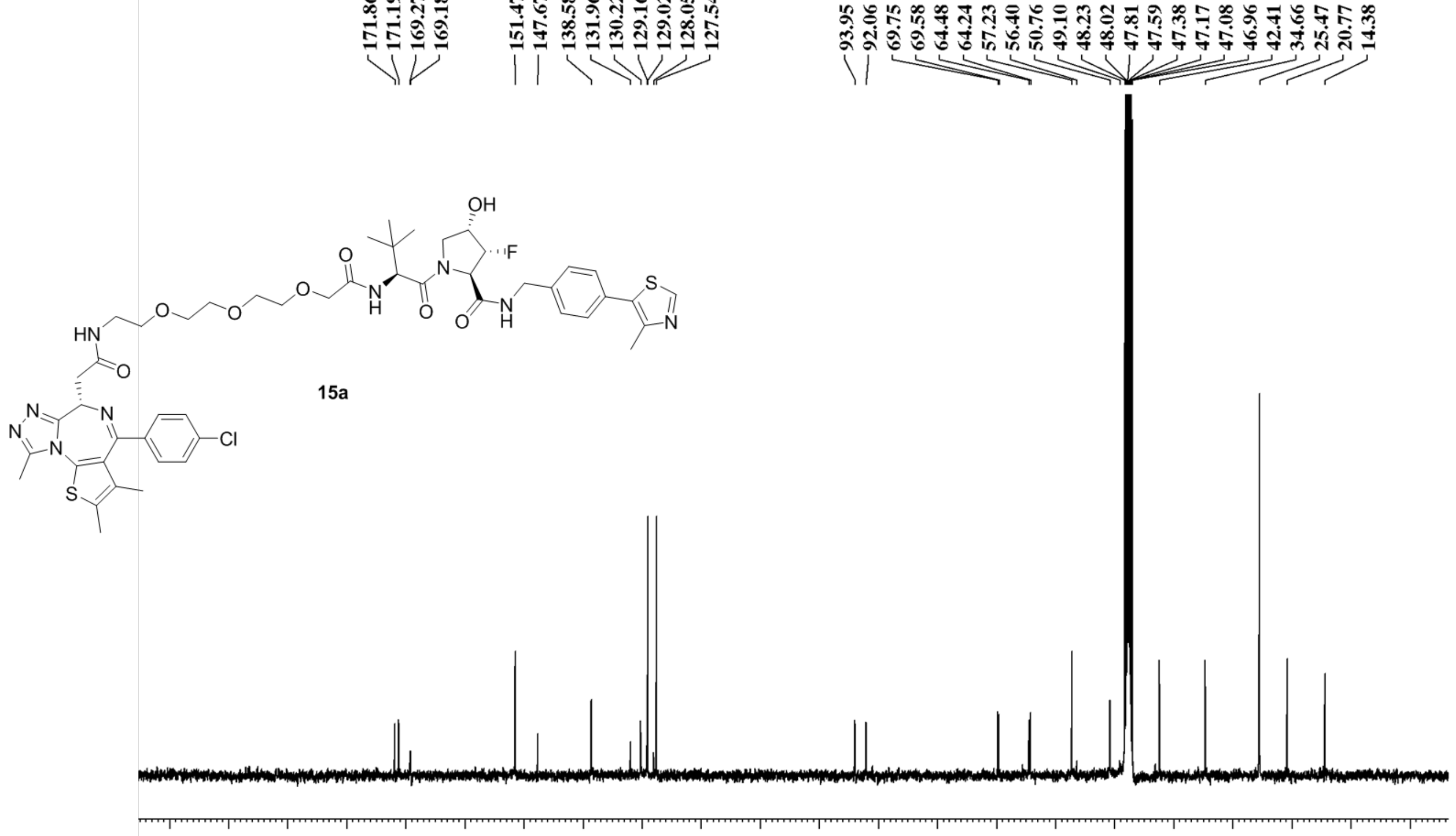




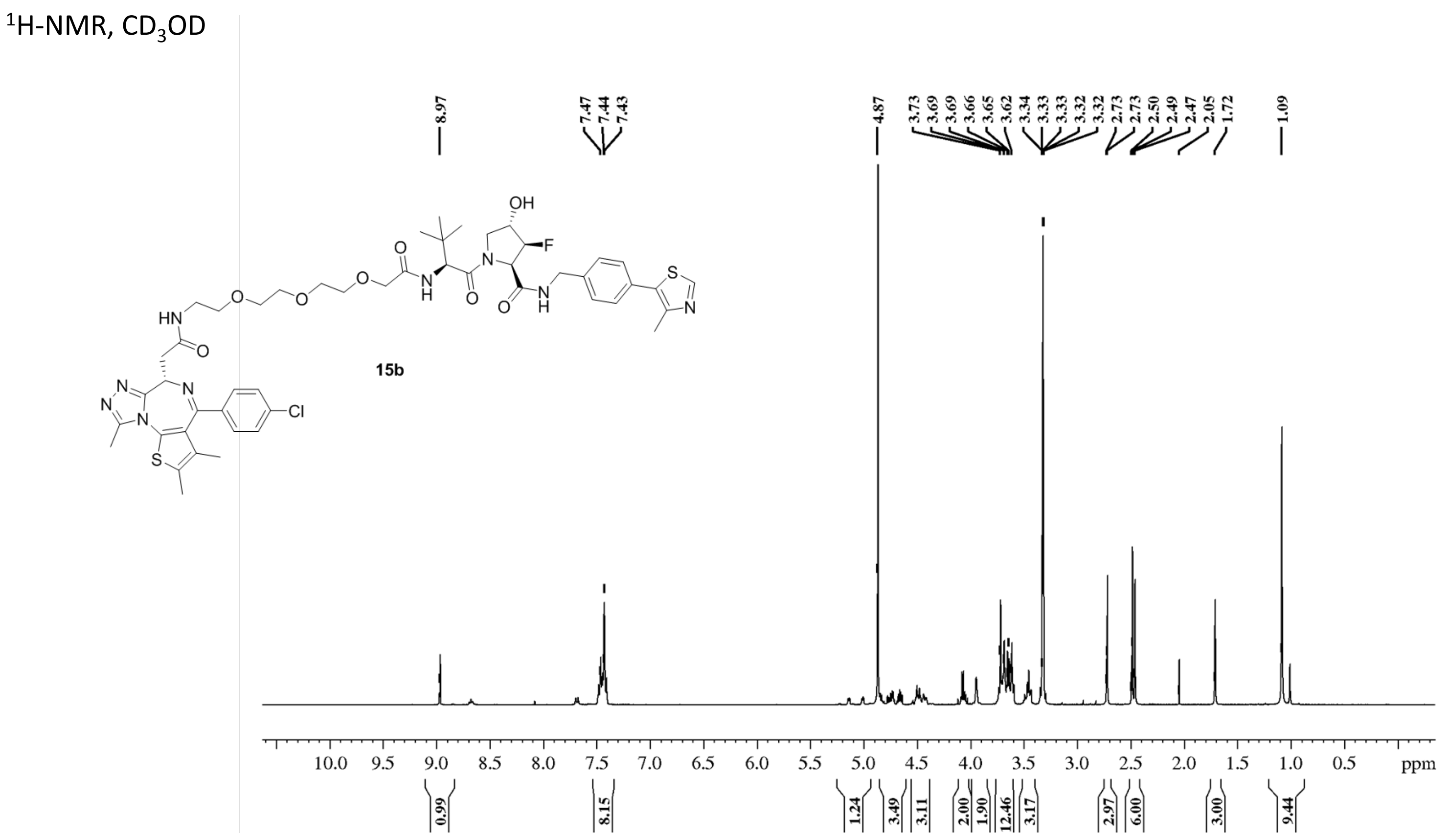


${ }^{19}$ F-NMR, $C D_{3} \mathrm{OD}$

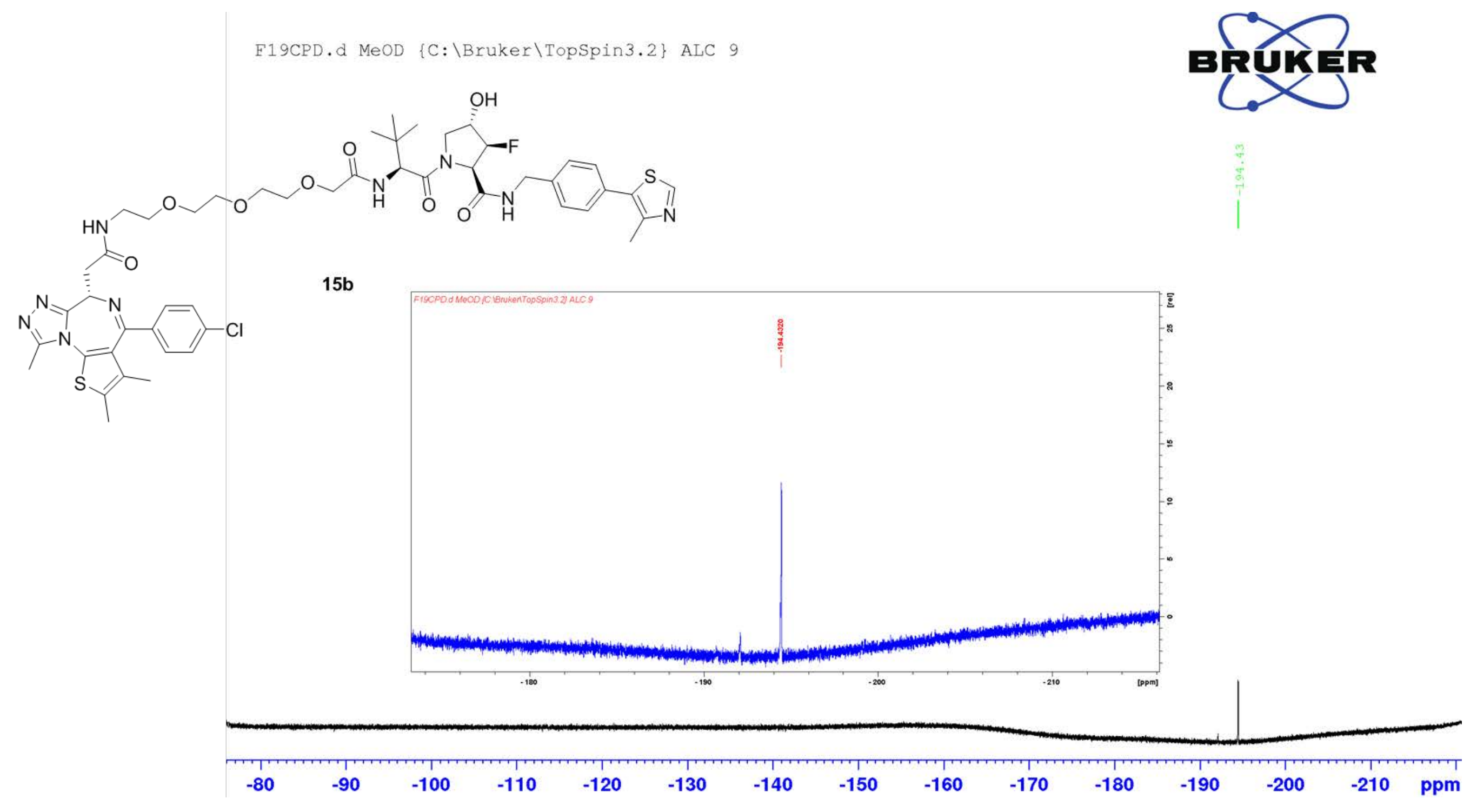

